Provided for non-commercial research and education use. Not for reproduction, distribution or commercial use.

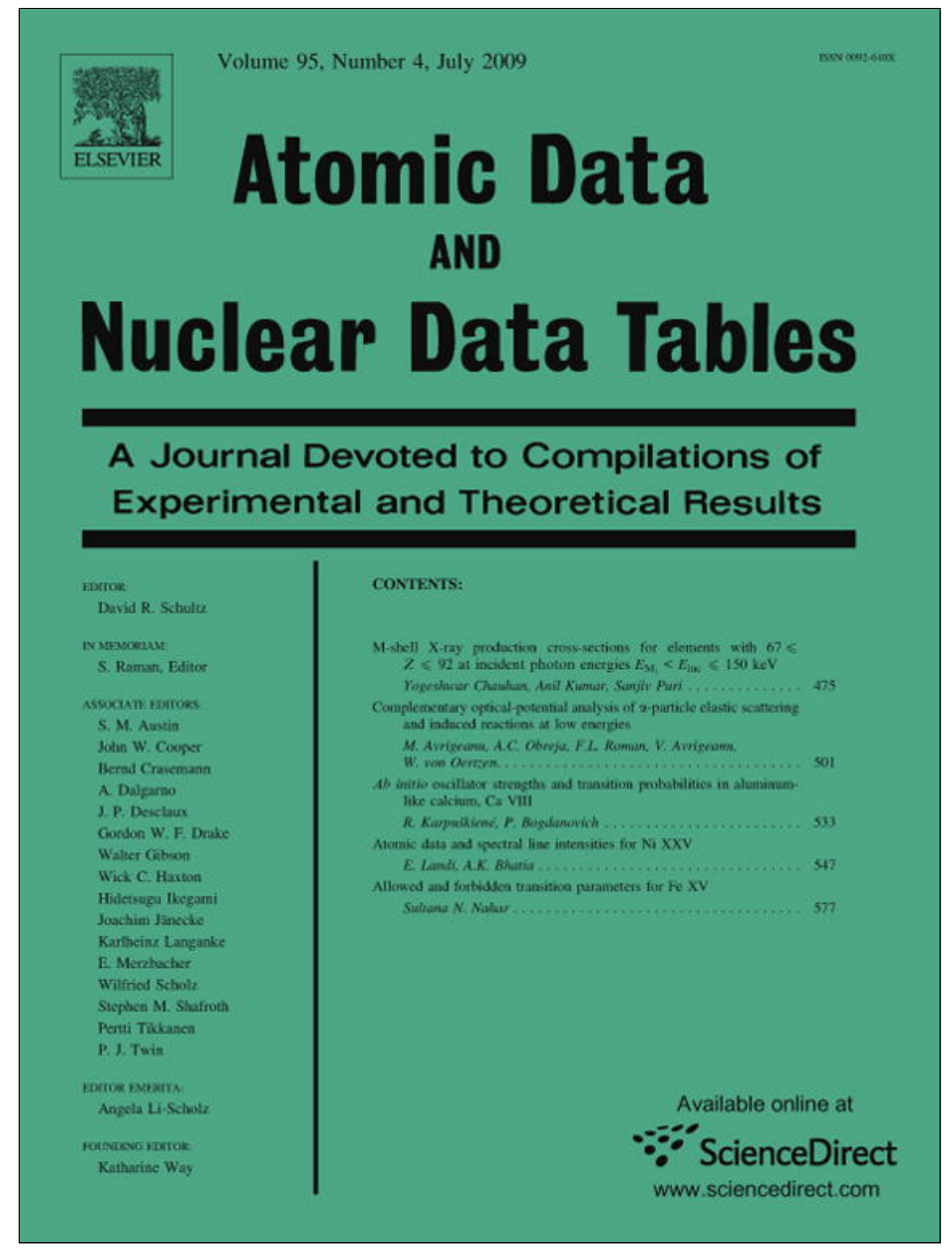

This article appeared in a journal published by Elsevier. The attached copy is furnished to the author for internal non-commercial research and education use, including for instruction at the authors institution and sharing with colleagues.

Other uses, including reproduction and distribution, or selling or licensing copies, or posting to personal, institutional or third party websites are prohibited.

In most cases authors are permitted to post their version of the article (e.g. in Word or Tex form) to their personal website or institutional repository. Authors requiring further information regarding Elsevier's archiving and manuscript policies are encouraged to visit:

http://www.elsevier.com/copyright 


\title{
Allowed and forbidden transition parameters for Fe XV
}

\author{
Sultana N. Nahar \\ Department of Astronomy, The Ohio State University, Columbus, OH 43210, USA
}

\section{A R T I C L E I N F O}

Article history:

Available online 21 April 2009

\begin{abstract}
A B S T R A C T
A comprehensive set of fine structure energy levels, oscillator strengths $(f)$, line strengths $(S)$, and radiative decay rates $(A)$ for bound-bound transitions in Fe XV is presented. The allowed electric dipole (E1) transitions were obtained from the relativistic Breit-Pauli R-matrix method which is based on the close coupling approximation. A total of 507 fine structure energy levels with $n \leqslant 10, l \leqslant 9$, and $0 \leqslant J \leqslant 10$ are found. They agree within $1 \%$ with the available observed energies. These energy levels yield a total of $27,812 \mathrm{E} 1$, same-spin multiplets and intercombination transitions. The $A$ values are in good agreement with those compiled by NIST and other existing values for most transitions. Forbidden transitions are obtained from a set of 20 configurations with orbitals ranging from $1 \mathrm{~s}$ to $5 \mathrm{f}$ using the relativistic code SUPERSTRUCTURE (SS) in the Breit-Pauli approximation. From a set of 123 fine structure levels, a total of $6962 S$ and $A$ values are presented for forbidden electric quadrupole (E2), electric octupole (E3), magnetic dipole (M1), and magnetic quadrupole (M2) transitions. The energies from SS calculations agree with observed energies to within 1-3\%. A values for E2, M1 transitions agree very well with the available values for most transitions while those for M2 transitions show variable agreement. The large set of transition parameters presented should be applicable for both diagnostics and spectral modeling in the X-ray, ultraviolet, and optical regions of astrophysical plasmas.
\end{abstract}

(c) 2009 Elsevier Inc. All rights reserved.

E-mail address: nahar@astronomy.ohio-state.edu 


\section{Contents}

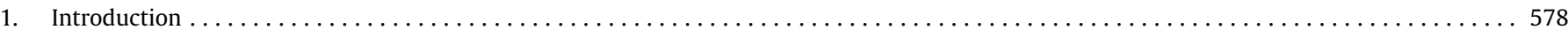

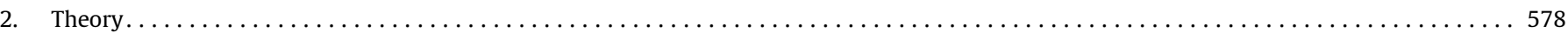

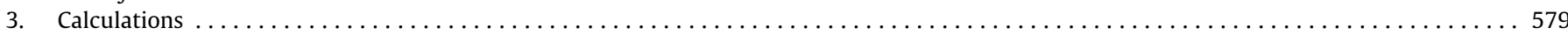

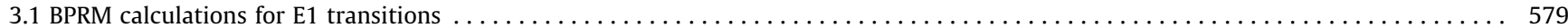

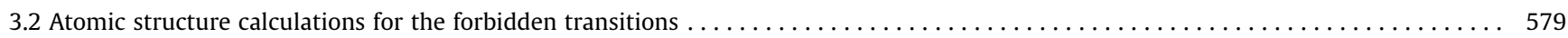

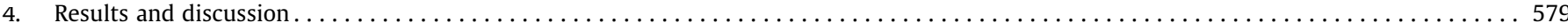

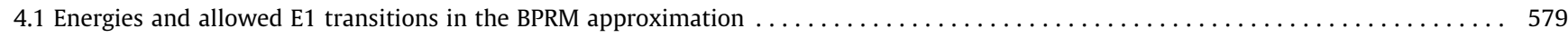

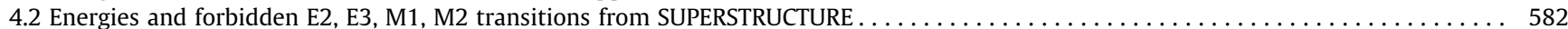

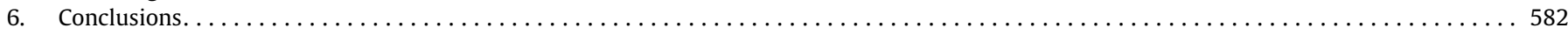

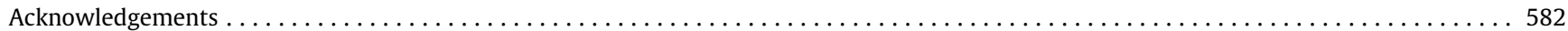

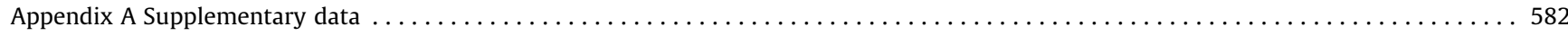

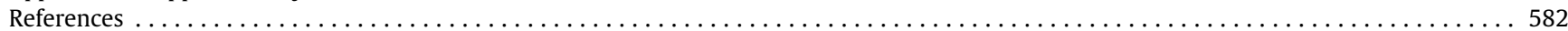

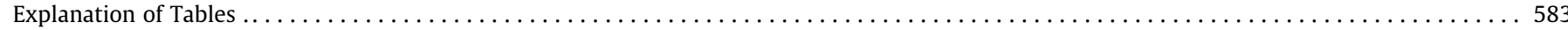
Tables

1. Partial set of 507 energy levels of Fe XV, grouped as fine structure components of $L S$ terms . . . . . . . . . . . . . . . . . 584

2. E1 transition probabilities for observed levels of Fe XV, grouped as fine structure components of $L S$ multiplets .......... 588

3. Fine structure energy levels of Fe XV for which forbidden (E2, E3, M1, M2) transitions are presented. . . . . . . . . . . . . 598

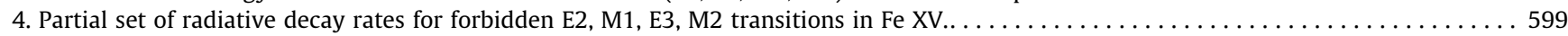

\section{Introduction}

Lines of the magnesium-like iron ion, $\mathrm{Fe} \mathrm{XV}$, with ground configuration $1 \mathrm{~s}^{2} 2 \mathrm{~s}^{2} 2 \mathrm{p}^{6} 3 \mathrm{~s}^{2}$, are commonly seen in astrophysical spectra. For example, emission lines in an extreme ultraviolet wavelength range of about 200-400 $\AA$ arising from $n=3-3$ transitions in Fe XV are widely detected in solar spectra (e.g., Ref. [1]). Soft X-ray emission lines of 50-80 ̊ due to $n=3-4$ transitions in $\mathrm{Fe} X \mathrm{XV}$ are seen in solar flares and the corona of Capella [2], and X-ray lines of 15-17 $\AA$ due to M-shell transitions (mainly 2p-3d) are seen in various objects, such as IRAS 13349-2438 [3].

Accurate atomic transition parameters are needed to extract conditions such as temperature, density, plasma outflow velocities, etc., of the astronomical objects. Earlier calculations of transition rates for Fe XV were carried out by Shorer et al. [4], Cowan and Widing [5], Cheng and Johnson [6], Anderson and Anderson [7], and Kastner et al. [8], which are included in an evaluated compilation carried out by Shirai et al. [9]. Calculations by Anderson and Anderson included both allowed and forbidden transitions. Transitions have been reported more recently by Deb et al. [10], Aggarwal et al. [11], Deb and Msezane [12], and Berrington et al. [13]. Results from all of these works correspond to various approximations based on atomic structure calculations. The present work reports the first large-scale $a b$ initio results for Fe XV from the relativistic Breit-Pauli R-matrix (BPRM) method aimed at improving astrophysical modeling.

\section{Theory}

The BPRM method is based on close coupling approximation where the atomic system is described as an N-electron target (core) interacting with a $(N+1)$ th electron. The wavefunction expansion, $\Psi_{E}$, of the $(N+1)$ electron system with total orbital angular momentum $L$, spin multiplicity $(2 S+1)$, and total angular momentum symmetry $J \pi$, is written as

$\Psi_{E}(\mathrm{e}+\mathrm{ion})=A \sum_{i} \chi_{i}($ ion $) \theta_{i}+\sum_{j} c_{j} \Phi_{j}(\mathrm{e}+\mathrm{ion})$,

where $\chi_{i}$ is the target ion wavefunction in a specific state $S_{i} L_{i} \pi_{i}$ or level $J_{i} \pi_{i}$, and $\theta_{i}$ is the wavefunction of the interacting $(N+1)$ th electron in a channel labeled as $S_{i} L_{i}\left(J_{i}\right) \pi_{i} k_{i}^{2} \ell_{i}(S L \pi$ or $J \pi)$ where $k_{i}^{2}$ is the incident kinetic energy. $\Phi_{j}$ s are correlation wavefunctions of the
$(N+1)$ electron system that (a) compensate for the orthogonality conditions between the continuum and the bound orbitals and (b) represent additional short-range correlation.

The relativistic Hamiltonian for the $(N+1)$ electron system in the Breit-Pauli approximation is given by (e.g., Ref. [15])

$$
\begin{aligned}
H_{N+1}^{\mathrm{BP}}= & H_{N+1}+H_{N+1}^{\mathrm{mass}}+H_{N+1}^{\mathrm{Dar}}+H_{N+1}^{s o}+\frac{1}{2} \sum_{i \neq j}^{N}\left[g_{i j}\left(s o+s o^{\prime}\right)\right. \\
& \left.+g_{i j}\left(s s^{\prime}\right)+g_{i j}\left(c s s^{\prime}\right)+g_{i j}(d)+g_{i j}\left(0 o^{\prime}\right)\right]
\end{aligned}
$$

where $H_{N+1}$ is the nonrelativistic Hamiltonian,

$H_{N+1}=\sum_{i=1}^{N+1}\left\{-\nabla_{i}^{2}-\frac{2 Z}{r_{i}}+\sum_{j>i}^{N+1} \frac{2}{r_{i j}}\right\}$.

$H_{N+1}^{\text {mass }}$ is the mass correction, $H_{N+1}^{\mathrm{Dar}}$ is the Darwin, and $H_{N+1}^{s o}$ is the spin-orbit interaction term, respectively. The two-body interaction terms are with notation $c$ for contraction, $d$ for Darwin, $o$ for orbit, $s$ for spin, and a prime indicates 'other.' All terms improve the energies except the spin-orbit interaction term which splits the energies into fine structure components. The BPRM Hamiltonian used in the Iron Project [16] includes the first three one-body corrections. However, the atomic structure calculations for the forbidden transitions include the contribution of the full Breit interaction term consisting of the fine structure terms, that is spin-other-orbit $\left(o s^{\prime}\right)$ and spinother-spin $\left(s s^{\prime}\right)$ terms, and ignores the last three two-body interaction terms.

In the BPRM method, the set of SL $\pi$ are recoupled to obtain (e + ion) states with total $J \pi$, following the diagonalization of the $(N+1)$-electron Hamiltonian,

$H_{N+1}^{B P} \Psi=E \Psi$.

Substitution of the wavefunction expansion results in a set of coupled equations that are solved using the R-matrix approach. At negative total energies $(E<0)$, the solutions of the close coupling equations occur at discrete eigenvalues of the $(e+$ ion $)$ Hamiltonian that correspond to pure bound states $\Psi_{B}$.

The oscillator strength $(f)$ for an electric dipole (E1) transition is proportional to the generalized line strength as

$S=\left|\left\langle\Psi_{f}\left|\sum_{j=1}^{N+1} r_{j}\right| \Psi_{i}\right\rangle\right|^{2}$ 
where $\Psi_{i}$ and $\Psi_{f}$ are the initial and final bound wavefunctions, respectively. The line strengths are energy independent quantities. This gives the oscillator strength, $f_{i j}$, and radiative decay rate or Einstein's A-coefficient (in atomic units, a.u.) as

$f_{i j}=\frac{E_{j i}}{3 g_{i}} S, \quad A_{j i}$ (a.u. $)=\frac{1}{2} \alpha^{3} \frac{g_{i}}{g_{j}} E_{j i}^{2} f_{i j}$.

$E_{j i}$ is the energy difference between the initial and final states, $\alpha$ is the fine structure constant, and $g_{i}, g_{j}$ are the statistical weight factors of the initial and final states, respectively.

The radiative decay rates for forbidden transitions are obtained from configuration interaction atomic structure calculations using the latest version of the program SUPERSTRUCTURE (SS) [17-19]. The transition rates for photo-excitations or de-excitations via various types of forbidden transitions can be obtained from the general line strength,

$S^{\mathrm{X} \lambda}(i j)=\left|\left\langle\Psi_{j}\left|O^{\mathrm{X} \lambda}\right| \Psi_{i}\right\rangle\right|^{2}, \quad S(j i)=S(i j)$.

where $\mathrm{X}$ represents the electric (E) or magnetic (M) type and $\lambda$ represents various multipoles, such as dipole (1), quadrupole (2), and octupole (3) [19]. The radiative decay rates for various higher orders of radiation are obtained from line strength $S^{\mathrm{X} \lambda}(i j)$ as follows.

Electric quadrupole (E2) and magnetic dipole (M1)

$g_{j} A_{j i}^{\mathrm{E} 2}=2.6733 \times 10^{3} \mathrm{~s}^{-1}\left(E_{j}-E_{i}\right)^{5} S^{\mathrm{E} 2}(i, j)$,

$g_{j} A_{j i}^{\mathrm{M} 1}=3.5644 \times 10^{4} \mathrm{~s}^{-1}\left(E_{j}-E_{i}\right)^{3} S^{\mathrm{M} 1}(i, j)$,

and for electric octopole (E3) and magnetic quadrupole (M2)

$g_{j} A_{j i}^{\mathrm{E} 3}=1.2050 \times 10^{-3} \mathrm{~s}^{-1}\left(E_{j}-E_{i}\right)^{7} S^{\mathrm{E} 3}(i, j)$,

$g_{j} A_{j i}^{\mathrm{M} 2}=2.3727 \times 10^{-2} \mathrm{~s}^{-1}\left(E_{j}-E_{i}\right)^{5} S^{\mathrm{M} 2}(i, j)$

The lifetime of a level can be obtained from the $A$ values as,

$\tau_{k}(s)=\frac{1}{\sum_{i} A_{k i}\left(s^{-1}\right)}$,

where the sum is the total radiative transition probability for the level $k$, and $A_{j i}\left(s^{-1}\right)=A_{j i}$ (a.u. $) / \tau_{0}, \tau_{0}=2.4191 \times 10^{-17} \mathrm{~s}$ is the atomic unit of time.

\section{Calculations}

\subsection{BPRM calculations for E1 transitions}

The BPRM calculation is initiated with the target (core) wavefunction which is obtained from an atomic structure calculation. Target Fe XVI wavefunctions were obtained from optimization of the 16 configurations, $2 p^{6} 3 s(1), 2 p^{6} 3 p(2), 2 p^{6} 3 d(3), 2 p^{6} 4 s(4)$, $2 \mathrm{p}^{6} 4 \mathrm{p}(5), 2 \mathrm{p}^{6} 4 \mathrm{~d}(6), 2 \mathrm{p}^{6} 4 \mathrm{f}(7), 2 \mathrm{p}^{5} 3 \mathrm{~s}^{2}(8), 2 \mathrm{p}^{5} 3 \mathrm{~s} 3 \mathrm{p}(9), 2 \mathrm{p}^{5} 3 \mathrm{~s} 3 \mathrm{~d}(10)$, $2 p^{5} 3 s 4 s(11), 2 p^{5} 3 s 4 p(12), 2 p^{5} 3 s 4 d(13), 2 p^{5} 3 s 4 f(14), 2 p^{5} 3 p^{2}(15)$, and $2 \mathrm{p}^{5} 3 \mathrm{p} 3 \mathrm{~d}(16)$ using the code SUPERSTRUCTURE [17]. All configurations are treated as spectroscopic. The values of Thomas-Fermi-Amaldi scaling parameters, $\lambda_{n l}$, for individual orbitals are 1.38(1s), 1.15(2s), 1.08(2p), 1.05(3s), 1.01(3p), 1.0(3d), 1.0(4s), $1.0(4 \mathrm{p}), 1.0(4 \mathrm{~d})$, and $1.0(4 \mathrm{f})$. The close coupling wavefunction expansion for Fe XV is formed from the first 12 levels of Fe XVI as given in Table A. The second bound-channel term of the wavefunction of Fe XV, Eq. (1), includes all possible $(N+1)$ configurations with filled $1 \mathrm{~s}$ and $2 \mathrm{~s}$ orbitals, and minimum and maximum occupancies, given within parentheses, in orbitals $2 \mathrm{p}(5-6), 3 \mathrm{~s}(0-$ 2), $3 \mathrm{p}(0-3), 3 \mathrm{~d}(0-2), 4 \mathrm{~s}(0-2), 4 \mathrm{p}(0-2), 4 \mathrm{~d}(0-1)$, and $4 \mathrm{f}(0-1)$.

Computations were carried out using the BPRM package of codes $[20,21]$. The general steps have been described in earlier papers [19]. Calculations included bound levels of Fe XV with
Table A

Levels and energies $\left(E_{t}\right)$ of the target Fe XVI in the wavefunction expansion of Fe XV. The present (SS) energies are compared with the observed energies in the NIST compilation [14].

\begin{tabular}{rllll}
\hline Level & & $J_{t}$ & $\begin{array}{l}E_{t}(\mathrm{Ry}) \\
\text { NIST }\end{array}$ & $\begin{array}{l}E_{t}(\mathrm{Ry}) \\
\text { SS }\end{array}$ \\
\hline 1 & $2 \mathrm{p}^{6} 3 \mathrm{~s}\left({ }^{2} \mathrm{~S}\right)$ & $1 / 2$ & 0.0 & 0. \\
2 & $2 \mathrm{p}^{6} 3 \mathrm{p}\left({ }^{2} \mathrm{p}^{o}\right)$ & $1 / 2$ & 2.52596 & 2.5371040 \\
3 & $2 \mathrm{p}^{6} 3 \mathrm{p}\left({ }^{2} \mathrm{p}^{o}\right)$ & $3 / 2$ & 2.71688 & 2.7176403 \\
4 & $2 \mathrm{p}^{6} 3 \mathrm{~d}\left({ }^{2} \mathrm{D}\right)$ & $3 / 2$ & 6.15544 & 6.1711038 \\
5 & $2 \mathrm{p}^{6} 3 \mathrm{~d}\left({ }^{2} \mathrm{D}\right)$ & $5 / 2$ & 6.1819 & 6.2092417 \\
6 & $2 \mathrm{p}^{6} 4 \mathrm{~s}\left({ }^{2} \mathrm{~S}\right)$ & $1 / 2$ & 17.0182 & 17.0188128 \\
7 & $2 \mathrm{p}^{6} 4 \mathrm{p}\left({ }^{2} \mathrm{p}^{o}\right)$ & $1 / 2$ & 18.0252 & 18.0162598 \\
8 & $2 \mathrm{p}^{6} 4 \mathrm{p}\left({ }^{2} \mathrm{p}^{o}\right)$ & $3 / 2$ & 18.098 & 18.0840405 \\
9 & $2 \mathrm{p}^{6} 4 \mathrm{~d}\left({ }^{2} \mathrm{D}\right)$ & $3 / 2$ & 19.3570 & 19.3520443 \\
10 & $2 \mathrm{p}^{6} 4 \mathrm{~d}\left({ }^{2} \mathrm{D}\right)$ & $5 / 2$ & 19.3677 & 19.3690583 \\
11 & $2 \mathrm{p}^{6} 4 \mathrm{f}\left({ }^{2} \mathrm{~F}^{o}\right)$ & $5 / 2$ & 19.9077 & 19.8951572 \\
12 & $2 \mathrm{p}^{6} 4 \mathrm{f}\left({ }^{2} \mathrm{~F}^{o}\right)$ & $7 / 2$ & 19.9125 & 19.9015720 \\
\hline
\end{tabular}

$0 \leqslant J \leqslant 10$ of even and odd parities, $n \leqslant 10, l \leqslant 9,0 \leqslant L \leqslant 12$, and $(2 S+1)=1,3$. The fine structure bound levels are obtained by scanning through the poles in the $(\mathrm{e}+\mathrm{ion})$ Hamiltonian with a fine mesh of effective quantum number $v$, at $\Delta v=0.001$ or smaller in code STGB.

The energies were then identified through a theoretical spectroscopy procedure as described in Ref. [22]. The bound-bound transitions from the BPRM code have been processed for energies and transition wavelengths using code PBPRAD. For levels that have been measured, the oscillator strengths and $A$ values, Eq. (6), were obtained from BPRM line strengths and the measured transition energies in order to improve the accuracy. This is a common practice of accuracy improvement, especially by the National Institute of Standards and Technology (NIST), since measured energies in general are more accurate than the calculated ones.

\subsection{Atomic structure calculations for the forbidden transitions}

Forbidden transitions of type E2, E3, M1, and M2 for Fe XV are obtained from an optimized set of 20 configurations with orbitals going up to $5 \mathrm{~g}$. All configurations are spectroscopic. The radiative decay rates were calculated from configuration interaction atomic structure calculations using the later version of SS that includes multipole transitions in the Breit-Pauli approximation [19]. The Thomas-Fermi-Amaldi $\lambda_{n l}$ parameters for the orbitals are 1.38(1s), 1.25(2s), 1.15(2p), 1.15(3s), 1.(3p), 1.0(3d), 1.0(4s), $1.0(4 \mathrm{p}), 1.0(4 \mathrm{~d}), 1.0(4 \mathrm{f}), 1.0(5 \mathrm{~s}), 1.0(5 \mathrm{p}), 1.0(5 \mathrm{~d}), 1.0(5 \mathrm{f})$, and $1.0(5 \mathrm{~g})$. The transitions have been processed by replacing the calculated energies by the available observed energies using the code PRCSS.

\section{Results and discussion}

A comprehensive set of oscillator strengths, line strengths, and radiative decay rates for allowed and forbidden transitions in Fe XV are presented. They are discussed in separate sections below.

\subsection{Energies and allowed E1 transitions in the BPRM approximation}

The allowed E1 transitions of Fe XV correspond to a large number, 507 in total, of fine structure energy levels with $n \leqslant 10$, $0 \leqslant l \leqslant 9$, and $0 \leqslant J \leqslant 10$ of even and odd parities.

The BPRM method finds energy eigenvalues without any identification. These energies have been identified spectroscopically through a procedure [22] that analyzes the quantum defects of levels, matches possible components of the relevant $L S$ terms, and con- 
siders channel percentage contributions to the integrated wavefunctions in the outer region. Hund's rule is also used for levels arising from the same configuration such that the level with higher orbital angular momentum $L$ and/or higher spin multiplicity will lie lower than those with lower $L$ and lower spin. Spectroscopic identification is a major task compared to atomic structure calculations where the levels are identified automatically. Based on the identification criteria, a level may have one or more possible designations due to mixing of states and similar quantum defects. These could introduce some uncertainties in the identification. Hence levels have been specified with all possible identifications. The identified designation provides information on the configuration, $L S$ term, and $J$ value of the core and $n l$ values of the outer or valence electron.

The complete set of 507 energy levels of Fe XV is available electronically. They are presented in two formats for various practical purposes: (i) in LSJ component format where fine structure levels are grouped as components of a $L S$ term and (ii) in a $J \pi$ set where levels of the symmetry are listed in ascending order of energy positions. A partial set of energies in format (i), useful for spectroscopic diagnostics, is given in Table 1. It provides the check for completeness of the set of energy levels that belong to the $L S$ term, and detects any missing level. Table B presents a partial set of energy levels in format (ii). The format is useful for modeling code applications.

The BPRM energies agree with those in the NIST table within $1 \%$ for all levels as shown in Table C. However, a large difference, 15\%, is found for the NIST identified level, $3 \mathrm{~s} 6 \mathrm{f}\left({ }^{1} \mathrm{~F}^{o}\right)$. It appears that the level might have been misidentified by NIST. The BPRM energies show similar agreement with other calculated energies. Berrington et al. [13] compare their calculated energies, in the Breit-Pauli and Dirac-Fock approximations, with those in the compiled table by NIST [14] as well as other existing calculated energies, such as by Deb et al. [10], Aggarwal et al. [11], and Deb and Msezane [12]. They obtained very good agreement with NIST, as in the case of the present work, and with other calculations. However, coming from atomic structure calculations, their energies are relative to the zero ground state, that is, the ground state is not specified whereas present energies are absolute.

The complete set of fine structure E1 transitions among the 507 levels consists of 27,812 E1 transitions. A sample set of transitions

\section{Table B}

Partial set of fine structure energy levels of Fe XV in $J \pi$ order. $i_{e}$ is the energy index and $I_{J}$ is the energy index of the level in its symmetry. There are 507 levels, $n \leqslant 10$, $l \leqslant 9$.

\begin{tabular}{|c|c|c|c|c|c|c|}
\hline$i_{e}$ & $J \pi$ & $I_{J}$ & $E(\mathrm{Ry})$ & Config & ${ }^{2 S+1} L^{\pi}$ & jjpiiii \\
\hline 1 & $0.0 \mathrm{e}$ & 1 & $-3.35800 \mathrm{E}+01$ & $2 p^{6} 3 s^{2}$ & ${ }^{1} S^{e}$ & 00001 \\
\hline 2 & $0.0 \mathrm{e}$ & 2 & $-2.85268 \mathrm{E}+01$ & $3 \mathrm{p}^{2}$ & ${ }^{3} \mathrm{P}^{e}$ & 00002 \\
\hline 3 & $0.0 \mathrm{e}$ & 3 & $-2.75690 \mathrm{E}+01$ & $3 p^{2}$ & ${ }^{1} S^{e}$ & 00003 \\
\hline 4 & $0.0 \mathrm{e}$ & 4 & $-2.06333 \mathrm{E}+01$ & $2 p^{6} 3 d^{2}$ & ${ }^{3} \mathrm{P}^{e}$ & 00004 \\
\hline 5 & $0.0 \mathrm{e}$ & 5 & $-2.00290 \mathrm{E}+01$ & $3 d^{2}$ & ${ }^{1} S^{e}$ & 00005 \\
\hline 6 & $0.0 \mathrm{e}$ & 6 & $-1.72957 \mathrm{E}+01$ & $3 s 4 s$ & ${ }^{1} S^{e}$ & 00006 \\
\hline 7 & $0.0 \mathrm{e}$ & 7 & $-1.36853 \mathrm{E}+01$ & $2 p^{6} 3 p^{2} p^{o} 4 p$ & ${ }^{3} \mathrm{P}^{e}$ & 00007 \\
\hline 8 & $0.0 \mathrm{e}$ & 8 & $-1.31260 \mathrm{E}+01$ & $2 p^{6} 3 p^{2} p^{0} 4 p$ & ${ }^{1} S^{e}$ & 00008 \\
\hline 9 & $0.0 \mathrm{e}$ & 9 & $-1.05409 \mathrm{E}+01$ & $2 p^{6} 3 s^{2} s^{e} 5 s$ & ${ }^{1} S^{e}$ & 00009 \\
\hline 10 & $0.0 \mathrm{e}$ & 10 & $-8.45982 \mathrm{E}+00$ & $2 p^{6} 3 d^{2} D^{e} 4 d$ & ${ }^{3} \mathrm{P}^{e}$ & 00010 \\
\hline 11 & $0.0 \mathrm{e}$ & 11 & $-8.16289 \mathrm{E}+00$ & $2 p^{6} 3 d^{2} D^{e} 4 d$ & ${ }^{1} S^{e}$ & 00011 \\
\hline 12 & $0.0 \mathrm{e}$ & 12 & $-7.50710 \mathrm{E}+00$ & $2 \mathrm{p}^{6} 3 \mathrm{p}^{2} \mathrm{P}^{0} 5 \mathrm{p}$ & ${ }^{3} \mathrm{P}^{e}$ & 00012 \\
\hline 13 & $0.0 \mathrm{e}$ & 13 & $-7.24559 \mathrm{E}+00$ & $2 \mathrm{p}^{6} 3 \mathrm{p}^{2} \mathrm{P}^{0} 5 \mathrm{p}$ & ${ }^{1} S^{e}$ & 00013 \\
\hline 14 & $0.0 \mathrm{e}$ & 14 & $-6.99588 \mathrm{E}+00$ & $2 p^{6} 3 s^{2} s^{e} 6 s$ & ${ }^{1} S^{e}$ & 00014 \\
\hline 15 & $0.0 \mathrm{e}$ & 15 & $-5.13427 \mathrm{E}+00$ & $2 p^{6} 3 s^{2} s^{e} 7 s$ & ${ }^{1} S^{e}$ & 00015 \\
\hline 16 & $0.0 \mathrm{e}$ & 16 & $-4.31644 \mathrm{E}+00$ & $2 p^{6} 3 p^{2} p^{o} 6 p$ & ${ }^{3} \mathrm{P}^{e}$ & 00016 \\
\hline 17 & $0.0 \mathrm{e}$ & 17 & $-4.07372 \mathrm{E}+00$ & $2 \mathrm{p}^{6} 3 \mathrm{p}^{2} \mathrm{P}^{0} 6 \mathrm{p}$ & ${ }^{1} S^{e}$ & 00017 \\
\hline 18 & $0.0 \mathrm{e}$ & 18 & $-3.86332 \mathrm{E}+00$ & $2 p^{6} 3 s^{2} s^{e} 8 s$ & ${ }^{1} S^{e}$ & 00018 \\
\hline 19 & $0.0 \mathrm{e}$ & 19 & $-3.14757 \mathrm{E}+00$ & $2 p^{6} 3 p^{2} p^{0} 7 p$ & ${ }^{3} \mathrm{P}^{e}$ & 00019 \\
\hline 20 & $0.0 \mathrm{e}$ & 20 & $-3.03995 \mathrm{E}+00$ & $2 \mathrm{p}^{6} 3 \mathrm{~s}^{2} \mathrm{~s}^{e} 9 \mathrm{~s}$ & ${ }^{1} S^{e}$ & 00020 \\
\hline 21 & $0.0 \mathrm{e}$ & 21 & $-2.97675 \mathrm{E}+00$ & $2 \mathrm{p}^{6} 3 \mathrm{p}^{2} \mathrm{P}^{0} 7 \mathrm{p}$ & ${ }^{1} S^{e}$ & 00021 \\
\hline 22 & $0.0 \mathrm{e}$ & 22 & $-2.44678 \mathrm{E}+00$ & $2 \mathrm{p}^{6} 3 \mathrm{p}^{2} \mathrm{p}^{0} 8 \mathrm{p}$ & ${ }^{3} \mathrm{P}^{e}$ & 00022 \\
\hline 23 & $0.0 \mathrm{e}$ & 23 & $-2.41103 \mathrm{E}+00$ & $2 p^{6} 3 s^{2} s^{e} 10 s$ & ${ }^{1} S^{e}$ & 00023 \\
\hline 24 & $0.0 \mathrm{o}$ & 1 & $-3.14491 \mathrm{E}+01$ & $3 s 3 p$ & ${ }^{3} \mathrm{P}^{o}$ & 10001 \\
\hline 25 & $0.0 \mathrm{o}$ & 2 & $-2.45048 \mathrm{E}+01$ & $3 p 3 d$ & ${ }^{3} \mathrm{P}^{o}$ & 10002 \\
\hline
\end{tabular}

Table C

Comparison of calculated BPRM energies, $E$, for Fe XV with observed values (NIST). $I_{J}$ is the calculated level index for its position in its $J \pi$ symmetry.

\begin{tabular}{|c|c|c|c|c|}
\hline Level & & $J: I_{J}$ & $E$ (Ry, NIST) & $E$ (Ry, BPRM) \\
\hline $2 p^{6} 3 s^{2}$ & ${ }^{1} \mathrm{~S}$ & $0.0: 1$ & $3.3580 \mathrm{E}+01$ & $3.3505 \mathrm{E}+01$ \\
\hline $3 s 3 p$ & ${ }^{3} \mathrm{P}^{o}$ & $2.0: 1$ & $3.1267 \mathrm{E}+01$ & $3.1195 E+01$ \\
\hline $3 s 3 p$ & ${ }^{3} \mathrm{P}^{o}$ & $1.0: 1$ & $3.1396 \mathrm{E}+01$ & $3.1320 \mathrm{E}+01$ \\
\hline $3 s 3 p$ & ${ }^{3} \mathrm{P}^{o}$ & $0.0: 1$ & $3.1449 \mathrm{E}+01$ & $3.1321 \mathrm{E}+01$ \\
\hline $3 s 3 p$ & ${ }^{1} \mathrm{P}^{o}$ & $1.0: 2$ & $3.0373 E+01$ & $3.0282 E+01$ \\
\hline $3 p^{2}$ & ${ }^{3} \mathrm{P}$ & $2.0: 1$ & $2.8278 \mathrm{E}+01$ & $2.8199 \mathrm{E}+01$ \\
\hline $3 \mathrm{p}^{2}$ & ${ }^{3} \mathrm{P}$ & $1.0: 1$ & $2.8435 \mathrm{E}+01$ & $2.8350 \mathrm{E}+01$ \\
\hline $3 \mathrm{p}^{2}$ & ${ }^{3} \mathrm{P}$ & $0.0: 2$ & $2.8527 \mathrm{E}+01$ & $2.8438 \mathrm{E}+01$ \\
\hline $3 p^{2}$ & ${ }^{1} \mathrm{D}$ & $2.0: 2$ & $2.8481 \mathrm{E}+01$ & $2.8398 \mathrm{E}+01$ \\
\hline $3 \mathrm{p}^{2}$ & ${ }^{1} \mathrm{~S}$ & $0.0: 3$ & $2.7569 \mathrm{E}+01$ & $2.7471 \mathrm{E}+01$ \\
\hline $3 s 3 d$ & ${ }^{3} \mathrm{D}$ & $3.0: 1$ & $2.7371 \mathrm{E}+01$ & $2.7267 \mathrm{E}+01$ \\
\hline $3 \mathrm{~s} 3 \mathrm{~d}$ & ${ }^{3} \mathrm{D}$ & $2.0: 3$ & $2.7385 \mathrm{E}+01$ & $2.7282 E+01$ \\
\hline $3 \mathrm{~s} 3 \mathrm{~d}$ & ${ }^{3} \mathrm{D}$ & $1.0: 2$ & $2.7395 \mathrm{E}+01$ & $2.7292 \mathrm{E}+01$ \\
\hline $3 \mathrm{~s} 3 \mathrm{~d}$ & ${ }^{1} \mathrm{D}$ & $2.0: 4$ & $2.6635 \mathrm{E}+01$ & $2.6518 \mathrm{E}+01$ \\
\hline $3 p 3 d$ & ${ }^{3} \mathrm{~F}^{o}$ & $4.0: 1$ & $2.4926 \mathrm{E}+01$ & $2.4825 E+01$ \\
\hline $3 \mathrm{p} 3 \mathrm{~d}$ & ${ }^{3} \mathrm{~F}^{o}$ & $3.0: 1$ & $2.5031 \mathrm{E}+01$ & $2.4928 \mathrm{E}+01$ \\
\hline $3 \mathrm{p} 3 \mathrm{~d}$ & ${ }^{3} \mathrm{~F}^{o}$ & $2.0: 2$ & $2.5121 \mathrm{E}+01$ & $2.5018 \mathrm{E}+01$ \\
\hline $3 p 3 d$ & ${ }^{1} \mathrm{D}^{o}$ & $2.0: 3$ & $2.4937 \mathrm{E}+01$ & $2.4835 E+01$ \\
\hline $3 p 3 d$ & ${ }^{3} \mathrm{D}^{\circ}$ & $3.0: 2$ & $2.4514 \mathrm{E}+01$ & $2.4405 E+01$ \\
\hline $3 \mathrm{p} 3 \mathrm{~d}$ & ${ }^{3} \mathrm{D}^{o}$ & $2.0: 4$ & $2.4498 \mathrm{E}+01$ & $2.4504 \mathrm{E}+01$ \\
\hline $3 p 3 d$ & ${ }^{3} \mathrm{D}^{o}$ & $1.0: 3$ & $2.4623 \mathrm{E}+01$ & $2.4511 \mathrm{E}+01$ \\
\hline $3 p 3 d$ & ${ }^{3} \mathrm{P}^{o}$ & $2.0: 5$ & $2.4618 \mathrm{E}+01$ & $2.4393 \mathrm{E}+01$ \\
\hline $3 p 3 d$ & ${ }^{3} \mathrm{P}^{o}$ & $1.0: 4$ & $2.4502 \mathrm{E}+01$ & $2.4394 \mathrm{E}+01$ \\
\hline $3 \mathrm{p} 3 \mathrm{~d}$ & ${ }^{3} \mathrm{P}^{o}$ & $0.0: 2$ & $2.4505 \mathrm{E}+01$ & $2.4394 \mathrm{E}+01$ \\
\hline $3 p 3 d$ & ${ }^{1} \mathrm{~F}^{o}$ & $3.0: 3$ & $2.3898 \mathrm{E}+01$ & $2.3779 \mathrm{E}+01$ \\
\hline $3 p 3 d$ & ${ }^{1} \mathrm{P}^{o}$ & $1.0: 5$ & $2.3785 \mathrm{E}+01$ & $2.3656 \mathrm{E}+01$ \\
\hline $3 d^{2}$ & ${ }^{3} \mathrm{~F}$ & $4.0: 1$ & $2.1059 \mathrm{E}+01$ & $2.0928 \mathrm{E}+01$ \\
\hline $3 d^{2}$ & ${ }^{3} \mathrm{~F}$ & $3.0: 2$ & $2.1077 \mathrm{E}+01$ & $2.0948 \mathrm{E}+01$ \\
\hline $3 d^{2}$ & ${ }^{3} \mathrm{~F}$ & $2.0: 5$ & $2.1093 \mathrm{E}+01$ & $2.0964 \mathrm{E}+01$ \\
\hline $3 d^{2}$ & ${ }^{1} \mathrm{D}$ & $2.0: 6$ & $2.0799 \mathrm{E}+01$ & $2.0662 \mathrm{E}+01$ \\
\hline $3 d^{2}$ & ${ }^{1} \mathrm{G}$ & $4.0: 2$ & $2.0758 \mathrm{E}+01$ & $2.0625 \mathrm{E}+01$ \\
\hline $3 d^{2}$ & ${ }^{3} \mathrm{P}$ & $2.0: 7$ & $2.0751 \mathrm{E}+01$ & $2.0612 \mathrm{E}+01$ \\
\hline $3 d^{2}$ & ${ }^{1} \mathrm{~S}$ & $0.0: 5$ & $2.0029 \mathrm{E}+01$ & $1.9861 \mathrm{E}+01$ \\
\hline $3 s 4 s$ & ${ }^{3} \mathrm{~S}$ & $1.0: 4$ & $1.7508 \mathrm{E}+01$ & $1.7435 \mathrm{E}+01$ \\
\hline
\end{tabular}

is presented in Table D. The complete file containing $f, S$, and $A$ values for $\mathrm{E} 1$ transitions is available electronically. In Table D, the top line specifies the nuclear charge $(Z=26)$ and number of electrons in the ion $\left(N_{e l c}=12\right)$. This line is followed by sets of oscillator strengths belonging to various pairs of symmetries $J_{i} \pi_{i}-J_{k} \pi_{k}$. The transition symmetries are expressed in the form of the statistical weight factors, $g=2 J+1$, and parity $\pi$ ( $=0$ for even and $=1$ for odd parity). $N_{i}, N_{f}$ are number of energies for the transitions symmetries and $N N$ is the number of transitions. Identification of the transition level indices $I_{i}$ and $I_{k}$ can be found from the energy Table 2. The third column in Table $D$ is the approximate transition wavelength $(\lambda)$ in $\AA$ obtained using $E(\AA)=911.2671 /\left(E_{i k}(\mathrm{Ry})\right.$. The fourth and fifth columns provide the energies $E_{i}$ and $E_{k}$ in Rydbergs of the transition levels. The sixth column is $f$, the oscillator strength in the length formulation. The sign of $f$ indicates the upper and lower levels in transitions such that a negative value means that $i$ is the lower level, while a positive value means $k$ is the lower level. Column seven is the line strength $S$, and the last column is the transition probability or the radiative decay rate $A_{k i}\left(\mathrm{~s}^{-1}\right)$. Calculated energies have been replaced by the observed energies wherever available for improved accuracy. Since the agreement of the calculated energies are within 1-3\% with the observed values, accuracy improvement could be attained to about $10 \%$ depending on the values of the energy levels.

A set of transitions employing only the observed set of levels has been processed with complete spectroscopic notation and grouped as fine structure components of $L S$ multiplets for direct comparison with experiment and other, such as, for diagnostic applications. The transitions are given in Table 2.

The present BPRM $A$ values for Fe XV are compared with the existing values, all obtained from atomic structure calculations, in Table 
Table D

Sample set of $f$-, $S$ and $A$-values for allowed E1 transitions in Fe XV. See text for explanation.

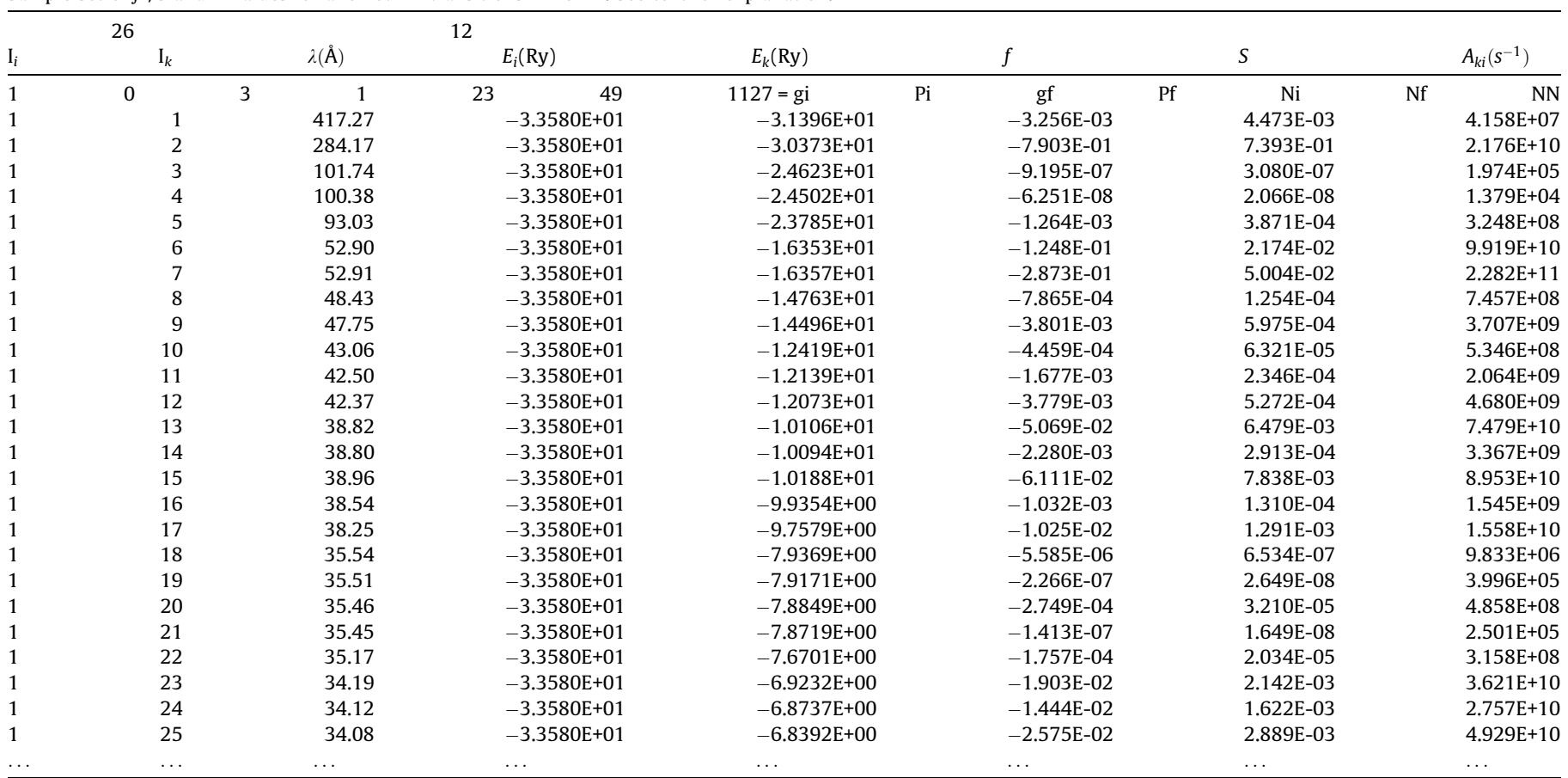

E. The $A$ values for $\mathrm{E} 1$ transitions using SS are also compared in Table E. The atomic structure calculations for the present forbidden transitions using SS also generated E1 transitions. This set is much smaller than the BPRM set. The NIST [14] table includes results from a number of authors and from the compiled table by Shirari et al. [9]. Recent calculations for the $A$ values were carried out by Deb et al. [10] using
CIV3 [23], Aggarwal et al. [11] and Deb and Msezane [12] using GRASP [24], and Berrington et al. [13] using AUTOSTRUCTURE, which is an adaptation of SS, as well as using Dirac-Fock GRASP code $[24,25]$. Comparison of the present BPRM $A$ values with SS as well as the existing values show very good agreement for most of the E1 transitions. However, significant differences are found for a few

Table E

Comparison of present $A$ values in units of $\mathrm{s}^{-1}$ for E1 transitions with those in the NIST compilation from Refs. [4](1), [5](2), [6](3), [7](4), [9](5), [8](6), and with Deb et al. [10](7), Berrington et al. [13](8) (a-Breit-Pauli atomic structure, b-Dirac atomic structure), Aggarwal et al. [11] and Deb et al. [10,12](9). The alphabetic letter is the NIST accuracy rating.

\begin{tabular}{|c|c|c|c|c|c|c|}
\hline \multirow[t]{2}{*}{$\lambda(\AA)$} & \multirow[t]{2}{*}{$A$ (Others) } & \multicolumn{2}{|c|}{$A$ (Present) } & \multirow[t]{2}{*}{$C_{i}-C_{j}$} & \multirow[t]{2}{*}{$S L \pi_{i}-S L \pi_{j}$} & \multirow[t]{2}{*}{$g_{i}-g_{j}$} \\
\hline & & BPRM & SS & & & \\
\hline 52.911 & $2.94 \mathrm{e}+11^{1}: C, 2.77 \mathrm{e}+11^{8 \mathrm{a}}, 2.95 \mathrm{e}+11^{8 \mathrm{~b}}, 2.29 \mathrm{e}+11^{7}$ & $2.28 \mathrm{e}+11$ & $2.38 \mathrm{e}+11$ & $2 p^{6} 3 s^{2}-3 s 4 p$ & ${ }^{1} \mathrm{~S}-{ }^{1} \mathrm{P}^{o}$ & $1-3$ \\
\hline 59.404 & $3.4 \mathrm{e}+11^{2}: C, 3.06 \mathrm{e}+11^{8 \mathrm{a}}, 3.14 \mathrm{e}+11^{8 \mathrm{~b}}, 2.49 \mathrm{e}+11^{7}$ & $2.40 \mathrm{e}+11$ & $2.23 e+11$ & $3 s 3 p-3 s 4 d$ & ${ }^{1} \mathrm{P}_{-}{ }^{1} \mathrm{D}$ & $3-5$ \\
\hline 307.73 & $4.91 \mathrm{e}+09^{3}: C, 5.09 \mathrm{e}+09^{8 \mathrm{a}}, 5.06 \mathrm{e}+09^{8 \mathrm{~b}}, 5.04 \mathrm{e}+09^{9}$ & $4.78 \mathrm{e}+09$ & $4.91 \mathrm{e}+09$ & $3 s 3 p-3 p^{2}$ & ${ }^{3} \mathrm{P}^{0}-{ }^{3} \mathrm{P}$ & $3-3$ \\
\hline 65.370 & $3.2 \mathrm{e}+10^{2}: C, 2.71 \mathrm{e}+10^{8 \mathrm{a}}, 3.34 \mathrm{e}+10^{8 \mathrm{~b}}$ & $3.70 \mathrm{e}+10$ & $3.52 \mathrm{e}+10$ & $3 s 3 p-3 s 4 s$ & ${ }^{3} \mathrm{P}^{o}-{ }^{3} \mathrm{~S}$ & $1-3$ \\
\hline 65.612 & $9.8 \mathrm{e}+10^{2}: C, 8.24 \mathrm{e}+10^{8 \mathrm{a}}, 1.02 \mathrm{e}+11^{8 \mathrm{~b}}$ & $1.14 \mathrm{e}+11$ & $1.06 \mathrm{e}+11$ & $3 s 3 p-3 s 4 s$ & ${ }^{3} \mathrm{P}^{0}-{ }^{3} \mathrm{~S}$ & $3-3$ \\
\hline 66.238 & $1.6 \mathrm{e}+11^{2}: C, 1.44 \mathrm{e}+11^{8 \mathrm{a}}, 1.72 \mathrm{e}+11^{8 \mathrm{~b}}$ & $1.95 \mathrm{e}+11$ & $1.82 \mathrm{e}+11$ & $3 s 3 p-3 s 4 s$ & ${ }^{3} \mathrm{P}^{0}-{ }^{3} \mathrm{~S}$ & $5-3$ \\
\hline 224.754 & $1.38 \mathrm{e}+10^{4}: C, 1.42 \mathrm{e}+10^{8 \mathrm{a}}, 1.42 \mathrm{e}+10^{8 \mathrm{~b}}, 1.36 \mathrm{e}+10^{7}$ & $1.35 \mathrm{e}+10$ & $1.40 \mathrm{e}+10$ & $3 s 3 p-3 s 3 d$ & ${ }^{3} \mathrm{P}^{o}-{ }^{3} \mathrm{D}$ & $1-3$ \\
\hline 227.206 & $1.8 \mathrm{e}+10^{4}: C, 1.86 \mathrm{e}+10^{8 \mathrm{a}}, 1.85 \mathrm{e}+10^{8 \mathrm{~b}}, 1.77 \mathrm{e}+10^{7}$ & $1.76 \mathrm{e}+10$ & $1.83 e+10$ & $3 s 3 p-3 s 3 d$ & ${ }^{3} \mathrm{P}^{0}-{ }^{3} \mathrm{D}$ & $3-5$ \\
\hline 227.734 & $9.8 \mathrm{e}+09^{4}: C, 1.02 \mathrm{e}+10^{8 \mathrm{a}}, 1.02 \mathrm{e}+10^{8 \mathrm{~b}}$ & $9.68 \mathrm{e}+09$ & $1.0 \mathrm{e}+10$ & $3 s 3 p-3 s 3 d$ & ${ }^{3} \mathrm{P}^{0}-{ }^{3} \mathrm{D}$ & $3-3$ \\
\hline 69.66 & $1.9 e+11^{5}: C, 2.55 e+11^{8 a}, 2.35 e+11^{8 b}$ & $2.18 \mathrm{e}+11$ & $2.16 e+11$ & $3 s 3 p-3 s 4 s$ & ${ }^{1} \mathrm{P}^{o}-{ }^{1} \mathrm{~S}$ & $3-1$ \\
\hline 243.794 & $4.2 \mathrm{e}+10^{4}: \mathrm{D}, 4.40 \mathrm{e}+10^{8 \mathrm{a}}, 4.41 \mathrm{e}+10^{8 \mathrm{~b}}, 4.36 \mathrm{e}+10^{7}$ & $4.09 \mathrm{e}+10$ & $4.25 \mathrm{e}+10$ & $3 s 3 p-3 s 3 d$ & ${ }^{1} \mathrm{P}^{o}-{ }^{1} \mathrm{D}$ & $3-5$ \\
\hline 63.96 & $1.6 \mathrm{e}+11^{2}: \mathrm{E}$ & $1.91 \mathrm{e}+11$ & $1.97 e+11$ & $3 p^{2}-3 s 4 f$ & ${ }^{1} \mathrm{D}-{ }^{1} \mathrm{~F}^{o}$ & $5-7$ \\
\hline 194.067 & $3.8 \mathrm{e}+08^{5}: E$ & $3.57 \mathrm{e}+08$ & $3.85 e+08$ & $3 p^{2}-3 p 3 d$ & ${ }^{1} \mathrm{D}-{ }^{1} \mathrm{P}^{o}$ & $5-3$ \\
\hline 231.68 & $1.5 \mathrm{e}+10^{5}: \mathrm{E}$ & $1.43 e+10$ & $1.58 \mathrm{e}+10$ & $3 p^{2}-3 p 3 d$ & ${ }^{3} \mathrm{P}-{ }^{3} \mathrm{P}^{o}$ & $3-3$ \\
\hline 231.87 & $2.1 \mathrm{e}+10^{5}: \mathrm{E}$ & $2.04 \mathrm{e}+10$ & $2.13 e+10$ & $3 p^{2}-3 p 3 d$ & ${ }^{3} \mathrm{P}-{ }^{3} \mathrm{P}^{o}$ & $3-1$ \\
\hline 242.100 & $2.3 e+10^{5}: D$ & $2.36 e+10$ & $2.56 \mathrm{e}+10$ & $3 p^{2}-3 p 3 d$ & ${ }^{3} \mathrm{P}_{-}{ }^{3} \mathrm{D}^{o}$ & $5-7$ \\
\hline 68.849 & $9.2 e^{+}+11^{6}: C$ & $9.15 \mathrm{e}+11$ & $8.66 e+11$ & $3 p 3 d-3 p 4 f$ & ${ }^{3} \mathrm{~F}^{\mathrm{o}}-{ }^{3} \mathrm{G}$ & $9-11$ \\
\hline 69.945 & $7.4 \mathrm{e}+11^{2}: C$ & $7.25 \mathrm{e}+11$ & $7.29 e+11$ & $3 s 3 d-3 s 4 f$ & ${ }^{3} \mathrm{D}-{ }^{3} \mathrm{~F}^{o}$ & $3-5$ \\
\hline 69.987 & $7.9 \mathrm{e}+11^{2}: C$ & $7.67 e+11$ & $7.72 \mathrm{e}+11$ & $3 s 3 d-3 s 4 f$ & ${ }^{3} \mathrm{D}-{ }^{3} \mathrm{~F}^{o}$ & $5-7$ \\
\hline 70.054 & $8.8 \mathrm{e}+11^{2}: C$ & $8.63 e+11$ & $8.70 \mathrm{e}+11$ & $3 s 3 d-3 s 4 f$ & ${ }^{3} \mathrm{D}-{ }^{3} \mathrm{~F}^{o}$ & $7-9$ \\
\hline 70.224 & $4.13 \mathrm{e}+11^{5}: C$ & $4.23 \mathrm{e}+11$ & $4.12 \mathrm{e}+11$ & $3 p 3 d-3 p 4 f$ & ${ }^{3} \mathrm{P}^{0}-{ }^{3} \mathrm{D}$ & $1-3$ \\
\hline 73.199 & $8.8 \mathrm{e}+11^{5}: C$ & $6.97 e+11$ & $7.95 e+11$ & $3 p 3 d-3 p 4 f$ & ${ }^{1} \mathrm{~F}^{o}-{ }^{1} \mathrm{G}$ & $7-9$ \\
\hline 73.473 & $6.2 \mathrm{e}+11^{2}: \mathrm{C}$ & $6.01 \mathrm{e}+11$ & $6.12 \mathrm{e}+11$ & $3 s 3 d-3 s 4 f$ & ${ }^{1} \mathrm{D}-{ }^{1} \mathrm{~F}^{o}$ & $5-7$ \\
\hline 312.556 & $1.1 \mathrm{e}+09^{3}: \mathrm{E}, 1.14 \mathrm{e}+09^{8 \mathrm{a}}, 1.09 \mathrm{e}+09^{8 \mathrm{~b}}, 1.08 \mathrm{e}+09^{9}$ & $1.16 \mathrm{e}+09$ & $1.01 \mathrm{e}+09$ & $3 s 3 p-3 p^{2}$ & ${ }^{3} \mathrm{P}^{o}-{ }^{1} \mathrm{D}$ & $3-5$ \\
\hline 238.114 & $3.2 \mathrm{e}+08^{4}: E, 2.88 \mathrm{e}+08^{8 \mathrm{a}}, 3.27 \mathrm{e}+08^{8 \mathrm{~b}}$ & $2.85 \mathrm{e}+08$ & $2.81 \mathrm{e}+08$ & $3 s 3 p-3 p^{2}$ & ${ }^{3} \mathrm{P}^{0}-{ }^{1} \mathrm{~S}$ & $3-1$ \\
\hline 191.408 & $3.5 e+08^{4}: E, 3.16 e+08^{8 a}, 3.67 e+08^{8 b}$ & $3.44 \mathrm{e}+08$ & $2.67 e+08$ & $3 s 3 p-3 s 3 d$ & ${ }^{3} \mathrm{P}^{o}-{ }^{1} \mathrm{D}$ & $3-5$ \\
\hline 196.741 & $1.6 \mathrm{e}+07^{4}: \mathrm{E}$ & $1.45 \mathrm{e}+07$ & $8.49 e+06$ & $3 s 3 p-3 s 3 d$ & ${ }^{3} \mathrm{P}^{o}-{ }^{1} \mathrm{D}$ & $5-5$ \\
\hline 304.998 & $3.0 e+07^{4}: E, 1.17 e+07^{8 a}, 1.73 e+07^{8 b}$ & $2.20 \mathrm{e}+07$ & $1.17 e+07$ & $3 s 3 p-3 s 3 d$ & ${ }^{1} \mathrm{P}^{o}-{ }^{3} \mathrm{D}$ & $3-5$ \\
\hline 305.889 & $2.6 \mathrm{e}+07^{4}: \mathrm{E}, 2.35 \mathrm{e}+07^{8 \mathrm{a}}, 2.42 \mathrm{e}+07^{8 \mathrm{~b}}$ & $2.63 e+07$ & $2.34 \mathrm{e}+07$ & $3 s 3 p-3 s 3 d$ & ${ }^{1} \mathrm{P}^{o}-{ }^{3} \mathrm{D}$ & $3-3$ \\
\hline 38.95 & $1.69 \mathrm{e}+11^{1}: C$ & $8.95 e+10$ & $1.56 \mathrm{e}+11$ & $2 p^{6} 3 s^{2}-3 s 5 p$ & ${ }^{1} \mathrm{~S}_{-}{ }^{1} \mathrm{P}^{0}$ & $1-3$ \\
\hline
\end{tabular}


Table F

Comparison of the present (SS) $A$ values for forbidden transitions with those in the NIST table. The alphabetic letter is the NIST accuracy (Ac) rating.

\begin{tabular}{llllll}
\hline$\lambda(\AA)$ & $\begin{array}{l}A: A c \\
\text { NIST }\end{array}$ & $\begin{array}{l}A\left(\mathrm{~s}^{-1}\right) \\
\mathrm{SS}\end{array}$ & $C_{i}-C_{j}$ & $S L \pi_{i}-S L \pi_{j}$ & $g_{i}-g_{j}$ \\
\hline E2,M1,M2 & & & & \\
131.216 & $1.6 \mathrm{e}+06: \mathrm{D}$ & $1.73 \mathrm{e}+06$ & $2 \mathrm{p}^{6} 3 \mathrm{~s}^{2}-3 \mathrm{~s} 3 \mathrm{~d}$ & ${ }^{1} \mathrm{~S}-{ }^{1} \mathrm{D}$ & $1-5 \mathrm{E} 2$ \\
171.913 & $4.3 \mathrm{e}+04^{1}: \mathrm{E}$ & $3.81 \mathrm{e}+04$ & $2 \mathrm{p}^{6} 3 \mathrm{~s}^{2}-3 \mathrm{p}^{2}$ & ${ }^{1} \mathrm{~S}-{ }^{3} \mathrm{P}$ & $1-5 \mathrm{E} 2$ \\
178.702 & $4.1 \mathrm{e}+05^{1}: \mathrm{E}$ & $1.59 \mathrm{e}+05$ & $2 \mathrm{p}^{6} 3 \mathrm{~s}^{2}-3 \mathrm{p}^{2}$ & ${ }^{1} \mathrm{~S}-{ }^{1} \mathrm{D}$ & $1-5 \mathrm{E} 2$ \\
20080 & $4.4 \mathrm{e}-01: \mathrm{E}$ & $4.13 \mathrm{e}-01$ & $3 \mathrm{p}^{2}-3 \mathrm{p}^{2}$ & ${ }^{1} \mathrm{D}-{ }^{3} \mathrm{P}$ & $5-3 \mathrm{M} 1$ \\
847.43 & $1.90 \mathrm{e}+02: \mathrm{E}$ & $1.70 \mathrm{e}+02$ & $3 \mathrm{~s} 3 \mathrm{p}-3 \mathrm{~s} 3 \mathrm{p}$ & ${ }^{3} \mathrm{P}^{o}-{ }^{1} \mathrm{P}^{o}$ & $1-3 \mathrm{M} 1$ \\
975.84 & $3.0 \mathrm{e}+01: \mathrm{E}$ & $2.70 \mathrm{e}+01$ & $3 \mathrm{p}^{2}-3 \mathrm{~s} 3 \mathrm{~d}$ & ${ }^{1} \mathrm{~S}-{ }^{1} \mathrm{D}$ & $1-5 \mathrm{E} 2$ \\
999.63 & $2.70 \mathrm{e}+02: \mathrm{E}$ & $2.70 \mathrm{e}+02$ & $3 \mathrm{p}^{2}-3 \mathrm{p}^{2}$ & ${ }^{1} \mathrm{D}-{ }^{1} \mathrm{~S}$ & $5-1 \mathrm{E} 2$ \\
1019.43 & $1.40 \mathrm{e}+02: \mathrm{E}$ & $1.24 \mathrm{e}+02$ & $3 \mathrm{~s} 3 \mathrm{p}-3 \mathrm{~s} 3 \mathrm{p}$ & ${ }^{3} \mathrm{P}^{o}-{ }^{1} \mathrm{P}^{o}$ & $5-3 \mathrm{M} 1$ \\
1052.00 & $1.400 \mathrm{e}+03: \mathrm{E}$ & $1.25 \mathrm{e}+03$ & $3 \mathrm{p}^{2}-3 \mathrm{p}^{2}$ & ${ }^{3} \mathrm{P}^{-}{ }^{1} \mathrm{~S}$ & $3-1 \mathrm{M} 1$ \\
1283.09 & $2.4 \mathrm{e}+01: \mathrm{E}$ & $2.13 \mathrm{e}+01$ & $3 \mathrm{p}^{2}-3 \mathrm{p}^{2}$ & ${ }^{3} \mathrm{P}-{ }^{1} \mathrm{~S}$ & $5-1 \mathrm{E} 2$ \\
224.278 & $1.2 \mathrm{e}+00: \mathrm{D}$ & 31.9 & $3 \mathrm{~s} 3 \mathrm{p}-3 \mathrm{~s} 3 \mathrm{~d}$ & ${ }^{3} \mathrm{P}^{o}-{ }^{3} \mathrm{D}$ & $1-5 \mathrm{M} 2$ \\
226.372 & $1.98 \mathrm{e}+00: \mathrm{C}$ & 26.3 & $3 \mathrm{~s} 3 \mathrm{p}-3 \mathrm{~s} 3 \mathrm{~d}$ & ${ }^{3} \mathrm{P}^{o}-{ }^{3} \mathrm{D}$ & $3-7 \mathrm{M} 2$ \\
246.423 & $4.2 \mathrm{e}+00: \mathrm{D}$ & 40.3 & $3 \mathrm{~s} 3 \mathrm{p}-3 \mathrm{p}^{2}$ & ${ }^{3} \mathrm{P}^{o}-{ }^{1} \mathrm{~S}$ & $5-1 \mathrm{M} 2$ \\
303.494 & $6.4 \mathrm{e}+00: \mathrm{C}$ & $7.88 \mathrm{e}+00$ & $3 \mathrm{~s} 3 \mathrm{p}-3 \mathrm{~s} 3 \mathrm{~d}$ & ${ }^{1} \mathrm{P}^{o}-{ }^{3} \mathrm{D}$ & $3-7 \mathrm{M} 2$ \\
393.980 & $3.39 \mathrm{e}+00: \mathrm{C}$ & $3.38 \mathrm{e}+00$ & $2 \mathrm{p}^{6} 3 \mathrm{~s}^{2}-3 \mathrm{~s} 3 \mathrm{p}$ & ${ }^{1} \mathrm{~S}-{ }^{3} \mathrm{P}^{o}$ & $1-5 \mathrm{M} 2$ \\
\hline & & & & &
\end{tabular}

transitions, such as $3 \mathrm{~s} 2\left({ }^{1} S_{0}\right)-3 \mathrm{~s} 5 \mathrm{p}\left({ }^{1} P_{1}^{o}\right)$, for which the BPRM $A$ value is much lower than that from NIST as well as SS.

\subsection{Energies and forbidden E2, E3, M1, M2 transitions from SUPERSTRUCTURE}

Forbidden transitions of type E2, E3, M1, and M2 are presented for 123 levels of 20 configurations with orbitals going up to $5 f$. Of these, 101 levels lie below the ionization threshold. These are obtained from the code SS which lists the levels in energy order regardless of their symmetry. Table 3 lists these levels processed with spectroscopic identification. The calculated energies agree with the measured values in the NIST compilation within less than $1 \%$ to a maximum of $3 \%$. They also show very good agreement with the 87-level calculations by Deb et al. [10], Aggarwal et al. [11], and Deb and Msezane [12], and 47-level calculations by Berrington et al. [13], all of which presented allowed transitions for a limited number of transitions. Small differences are expected since the set of configurations and relevant parameters are different for each calculation. The present calculations included higher levels than the earlier calculations.

The forbidden transitions with the observed levels have been reprocessed with calculated line strengths and observed energies to obtain the $A$ values. The energies in Table 3 correspond to energies used to process the $A$ values of the forbidden transitions. Hence they are combinations of observed (whenever available) and calculated energies, and calculated energies (when no observed values are available).

$S$ and $A$ values for a total of 6962 forbidden transitions of type E2, E3, M1, and M2 have been found for Fe XV. Table 4 presents a partial set of these transitions. The parity remains unchanged for the $\mathrm{E} 2$ and M1 transitions and hence are presented together. On the other hand, parity changes for E3 and M2 transitions which are presented together. The complete set of transitions processed from SS output with standard spectroscopic notation is available electronically.

Comparison of the present $A$ values are made with those available in the NIST compilation, computed by Anderson and Anderson [7], in Table F. Good agreement is found between SS and NIST for E2, M1 transitions. Variable agreement is noted between SS and NIST for M2 transitions.

\section{Conclusions}

Photo-excitations and de-excitations of Fe XV are studied in detail and an extensive set of transition parameters for oscillator strengths, line strengths, and radiative decay rates is presented for both allowed and forbidden transitions. The allowed transitions are treated with a large-scale relativistic Breit-Pauli R-matrix method and the forbidden transitions are treated with relativistic atomic structure calculations using the code SUPERSTRUCTURE. Very good agreement, within $1 \%$, is found for the BPRM calculated energies with the measured values while those from SS vary from less than $1-3 \%$.

The BPRM E1 transition rates are in very good agreement with available results for most transitions. The advantage of the present BPRM method is its capability of including much more configuration interactions than that of the atomic structure calculations. However the atomic structure calculations in the Breit-Pauli approximation can include two-body interaction terms and the Dirac-Fock method can make better optimization of the energies. For highly charged $\mathrm{Fe}$ $\mathrm{XV}$, the 47-level calculations by Berrington et al. [13], the 87-level calculations by Aggarwal et al. [11] and Deb and Msezane [12], and the present calculations provide similar results for most of the transitions. Some differences in the results are expected from differences in the methodologies. The present forbidden transitions compare very well with those in the NIST compilation for most transitions. However, M2 transitions have variable agreement with others. The present results are expected to be accurate and complete enough for most astrophysical and laboratory applications. In addition to the present supplemental data files, all data are available electronically at the NORAD website: www.astronomy.ohio-state.edu/ nahar/nahar_radiativeatomicdata/index.html

\section{Acknowledgements}

This work was partially supported by the NASA APRA program. The computational work was carried out on the Cray X1 at the Ohio Supercomputer Center in Columbus, Ohio.

\section{Appendix A. Supplementary data}

Supplementary data associated with this article can be found, in the online version, at doi:10.1016/j.adt.2009.03.002.

\section{References}

[1] K.P. Dere, Astrophys. J. 221 (1978) 1062

[2] F.P. Keenan, J.J. Drake, S. Chung, N.S. Brickhouse, K.M. Aggarwal, A.Z. Msezane, R.S.I. Ryans, D.S. Bloomfeld, Astrophys. J. 645 (2006) 597.

[3] M. Sako, S.M. Kahn, E. Behar, J.S. Kaastra, A.C. Brinkman, Th. Boller, E.M. Puchnarewicz, R. Starling, D.A. Liedahl, J. Clavel, M. Santos-Lleo, Astron. Astrophys. 365 (2001) L168

[4] P. Shorer, C.D. Lin, W.R. Johnson, Phys. Rev. A 16 (1977) 1109

[5] R.D. Cowan, K.G. Widing, Astrophys. J. 180 (1973) 285.

[6] K.T. Cheng, W.R. Johnson, Phys. Rev. A 16 (1977) 263.

[7] E.K. Anderson, E.M. Anderson, Opt. Spectrosc. (USSR) 55 (1983) 500.

[8] S.O. Kastner, M. Swartz, A.K. Bhatia, J. Lapides, J. Opt. Soc. Am. 68 (1978) 1558

[9] T. Shirai, J. Sugar, A. Musgrove, W.L. Wiese, J. Phys. Chem. Ref. Data, Monograph No. 8 (2000).

[10] N.C. Deb, K.M. Aggarwal, A.Z. Msezane, Astrophys. J. Suppl. 121 (1999) 265

[11] K.M. Aggarwal, N.C. Deb, F.P. Keenen, A.Z. Msezane, J. Phys. B 32 (1999) 5257

[12] N.C. Deb, A.Z. Msezane, J. Phys. B 31 (1998) L281.

[13] K.A. Berrington, C.P. Ballance, D.C. Griffin, N.R. Badnell, J. Phys. B 38 (2005) 1667.

[14] National Institute for Standards and Technology (NIST), compilations of atomic data are available at http://physics.nist.gov/PhysRefData/ASD/index.html.

[15] W. Eissner, in: S. Wilson et al. (Eds.), The Effects of Relativity on Atoms, Molecules, and the Solid State, Plenum Press, New York, 1991, p. 55.

[16] D.G. Hummer, K.A. Berrington, W. Eissner, A.K. Pradhan, H.E. Saraph, J.A. Tully, Astron. Astrophys. 279 (1993) 298.

[17] W. Eissner, M. Jones, H. Nussbaumer, Comput. Phys. Commun. 8 (1974) 270.

[18] W. Eissner, C.J. Zeippen, J. Phys. B 14 (1981) 2125.

[19] S.N. Nahar, W. Eissner, G.X. Chen, A.K. Pradhan, Astron. Astrophys. 408 (2003) 789.

[20] K.A. Berrington, P.G. Burke, K. Butler, M.J. Seaton, P.J. Storey, K.T. Taylor, Yu. Yan, J. Phys. B 20 (1987) 6379.

[21] K.A. Berrington, W. Eissner, P.H. Norrington, Comput. Phys. Commun. 92 (1995) 290.

[22] S.N. Nahar, A.K. Pradhan, Phys. Scr. 61 (2000) 675

[23] A. Hibbert, Comput. Phys. Commun. 9 (1975) 141

[24] K.G. Dyall, I.P. Grant, C.T. Johnson, F.A. Parpia, E.P. Plummer, Comput. Phys Commun. 55 (1989) 425

[25] P.H. Norrington, 2004. Available from: http://web.am.qub.ac.uk/DARC (Currently, in 2009, is unavailable due to publication preparation reason). 


\section{Explanation of Tables}

Table 1. Partial set of 507 energy levels of Fe XV, grouped as fine structure components of $L S$ terms.

The levels are designated as $C_{t}\left(S_{t} L_{t} \pi_{t}\right) J_{t} n l J(S L) \pi$

The top line of each set provides the expected number of fine structure levels (Nlv) for the possible ${ }^{(2 S+1)} L^{\pi}$ terms with the given configurations. In the set, the spin multiplicity $(2 S+1)$ and parity $\pi$ are fixed, but $L$ varies. Within parenthesis next to each $L$, all possible $J$ values associated with the given $L S$ term are specified. This line is followed by a set of the energy levels of the same configurations. $\mathrm{Nlv}(\mathrm{c})$ at the end specifies the total number of calculated $J$ levels found for the set. If $\mathrm{Nlv}=\operatorname{Nlv}(\mathrm{c})$, the calculated energy set for the given terms is complete

The levels are designated as $C_{t}\left(S_{t} L_{t} \pi_{t}\right) J_{t} n l J(S L) \pi$

$C_{t} \quad$ target configuration

$S_{t} L_{t} \pi_{t} \quad S L \pi$ symmetry of the target

$J_{t} \quad$ total angular momentum of the target state

$\mathrm{nl} \quad$ configuration of the valence electron

$J \quad$ total angular momentum of the level

$E$ (Ry) level energy in Rydberg

$v \quad$ effective quantum number

SL $\pi \quad$ symmetry of the level

Table 2. E1 transition probabilities for observed levels of Fe XV, grouped as fine structure components of $L S$ multiplets. The calculated transition energies have been replaced by the observed energies.

$C_{i}-C_{k} \quad$ configurations of transition levels

$T_{i}-T_{k} \quad L S$ term designation of the level

$g_{i} \quad$ statistical weight factor $(2 J+1)$ of the level

I position of the level in its SL $\pi$ symmetry

$E_{i} k(\AA) \quad$ transition energy (in Angstroms)

$f, S, A\left(\mathrm{~s}^{-1}\right)$ oscillator strength, line strength, radiative decay rate

Table 3. Fine structure energy levels of Fe XV for which forbidden (E2, E3, M1, M2) transitions are presented. The indices (cf) correspond to configurations $2 p^{6} 3 s^{2}(1), 2 p^{6} 3 s 3 p(2), 2 p^{6} 3 p^{2}(3), 2 p^{6} 3 s 3 d(4), 2 p^{6} 3 p 3 d(5), 2 p^{6} 3 s 4 s(6)$, $2 \mathrm{p}^{6} 3 \mathrm{~s} 4 \mathrm{p}(7), 2 \mathrm{p}^{6} 3 \mathrm{~s} 4 \mathrm{~d}(8), 2 \mathrm{p}^{6} 4 \mathrm{~s} 4 \mathrm{f}(9), 2 \mathrm{p}^{6} 3 \mathrm{~s} 5 \mathrm{~s}(10), 2 \mathrm{p}^{6} 3 \mathrm{~s} 5 \mathrm{p}(11), 2 \mathrm{p}^{6} 3 \mathrm{~s} 5 \mathrm{~d}(12), 2 \mathrm{p}^{6} 3 \mathrm{p} 4 \mathrm{~s}(13), 2 \mathrm{p}^{6} 3 \mathrm{p} 4 \mathrm{p}(14), 2 \mathrm{p}^{6} 3 \mathrm{p} 4 \mathrm{~d}(15)$, $2 \mathrm{p}^{6} 3 \mathrm{p} 4 \mathrm{f}(16), 2 \mathrm{p}^{5} 3 \mathrm{~s}^{2} 3 \mathrm{p}(17), 2 \mathrm{p}^{5} 3 \mathrm{~s}^{2} 3 \mathrm{~d}(18), 2 \mathrm{p}^{6} 3 \mathrm{~d}^{2}(19), 2 \mathrm{p}^{6} 3 \mathrm{~s} 5 \mathrm{f}(20)$

$i_{e} \quad$ level index

SLp(cf) LS term of the level and configuration number cf

2J $J$ is total angular momentum

$E$ (Ry) relative energy in Rydberg of the level

Table 4. Partial set of radiative decay rates for forbidden E2, M1, E3, M2 transitions in Fe XV.

The indices (cf) correspond to configurations $2 \mathrm{p}^{6} 3 \mathrm{~s}^{2}(1), 2 \mathrm{p}^{6} 3 \mathrm{~s} 3 \mathrm{p}(2), 2 \mathrm{p}^{6} 3 \mathrm{p}^{2}(3), 2 \mathrm{p}^{6} 3 \mathrm{~s} 3 \mathrm{~d}(4), 2 \mathrm{p}^{6} 3 \mathrm{p} 3 \mathrm{~d}(5), 2 \mathrm{p}^{6} 3 \mathrm{~s} 4 \mathrm{~s}(6)$, $2 \mathrm{p}^{6} 3 \mathrm{~s} 4 \mathrm{p}(7), 2 \mathrm{p}^{6} 3 \mathrm{~s} 4 \mathrm{~d}(8), 2 \mathrm{p}^{6} 4 \mathrm{~s} 4 \mathrm{f}(9), 2 \mathrm{p}^{6} 3 \mathrm{~s} 5 \mathrm{~s}(10), 2 \mathrm{p}^{6} 3 \mathrm{~s} 5 \mathrm{p}(11), 2 \mathrm{p}^{6} 3 \mathrm{~s} 5 \mathrm{~d}(12), 2 \mathrm{p}^{6} 3 \mathrm{p} 4 \mathrm{~s}(13), 2 \mathrm{p}^{6} 3 \mathrm{p} 4 \mathrm{p}(14), 2 \mathrm{p}^{6} 3 \mathrm{p} 4 \mathrm{~d}(15)$, $2 \mathrm{p}^{6} 3 \mathrm{p} 4 \mathrm{f}(16), 2 \mathrm{p}^{5} 3 \mathrm{~s}^{2} 3 \mathrm{p}(17), 2 \mathrm{p}^{5} 3 \mathrm{~s}^{2} 3 \mathrm{~d}(18), 2 \mathrm{p}^{6} 3 \mathrm{~d}^{2}(19), 2 \mathrm{p}^{6} 3 \mathrm{~s} 5 \mathrm{f}(20)$

$N_{t r} \quad$ total number of transitions

$i-j \quad$ energy indices of the levels as given in Table 3

$N_{i} \quad L S$ term designation of the level

$C_{i} \quad$ configuration number of the transition level

$g_{i} \quad$ statistical weight factor $(2 J+1)$ of the level

$\lambda$ transition energy. Note that for extremely low energy, $\lambda=10,000 \AA$

$E_{i}, E_{f}$ energies of the levels

$A E 2$ radiative decay rate for $\mathrm{E} 2$ transition

$A E 3$ radiative decay rate for $\mathrm{E} 3$ transition

$A M 1$ radiative decay rate for M1 transition

AM2 radiative decay rate for $\mathrm{M} 2$ transition 
Table 1

Partial set of 507 energy levels of Fe XV, grouped as fine structure components of $L S$ terms. See page 583 for Explanation of Tables.

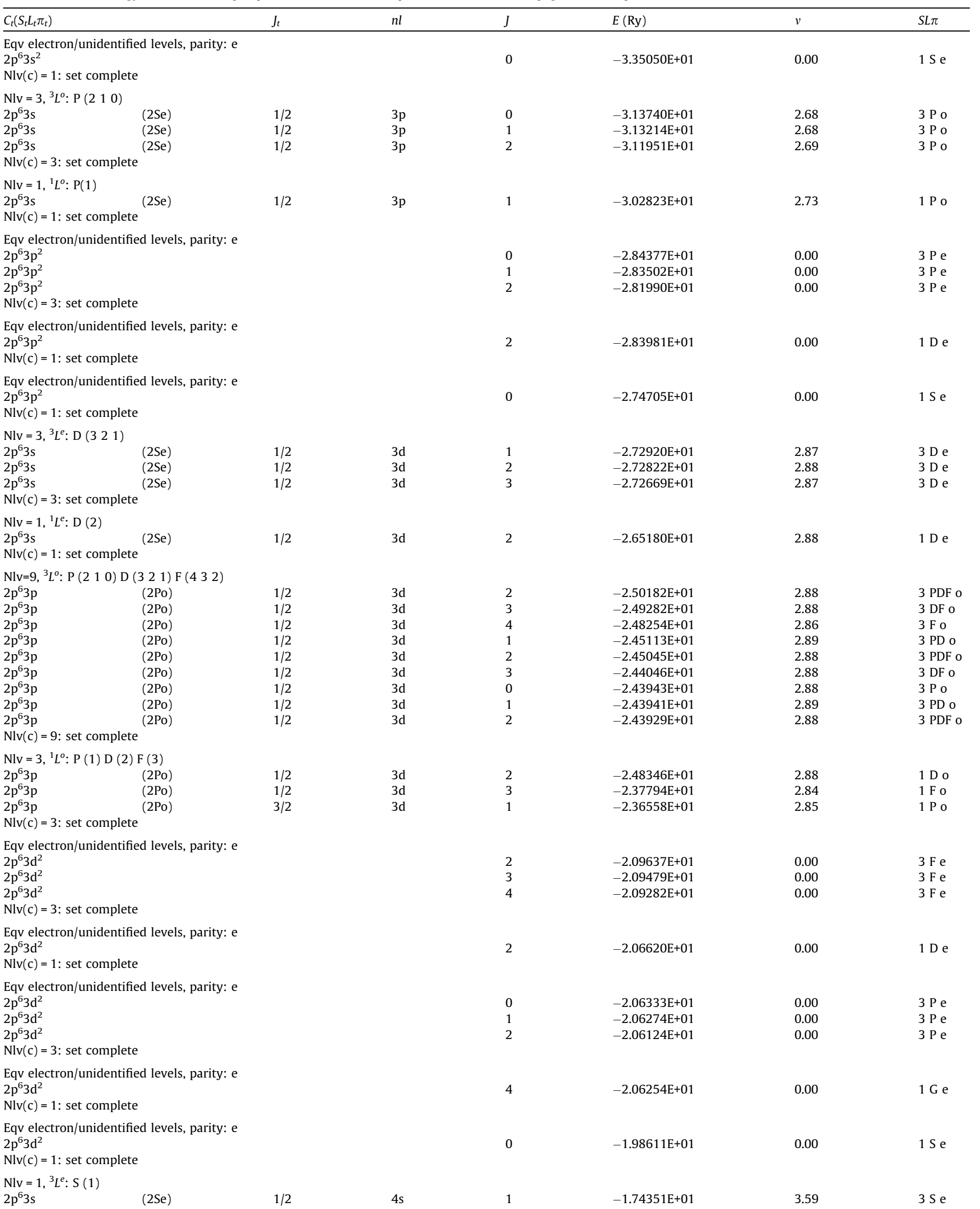


Table 1. (continued)

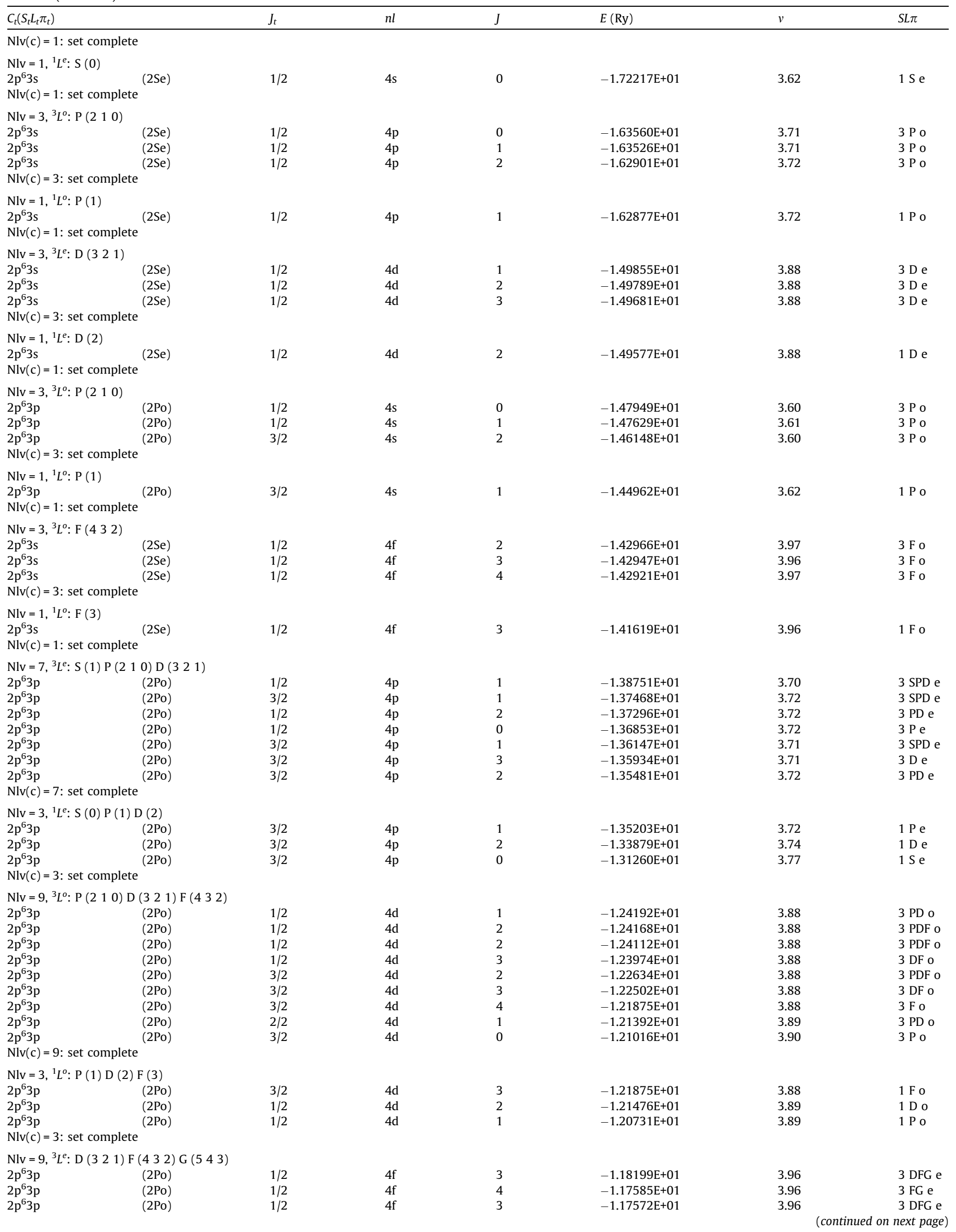


Table 1. (continued)

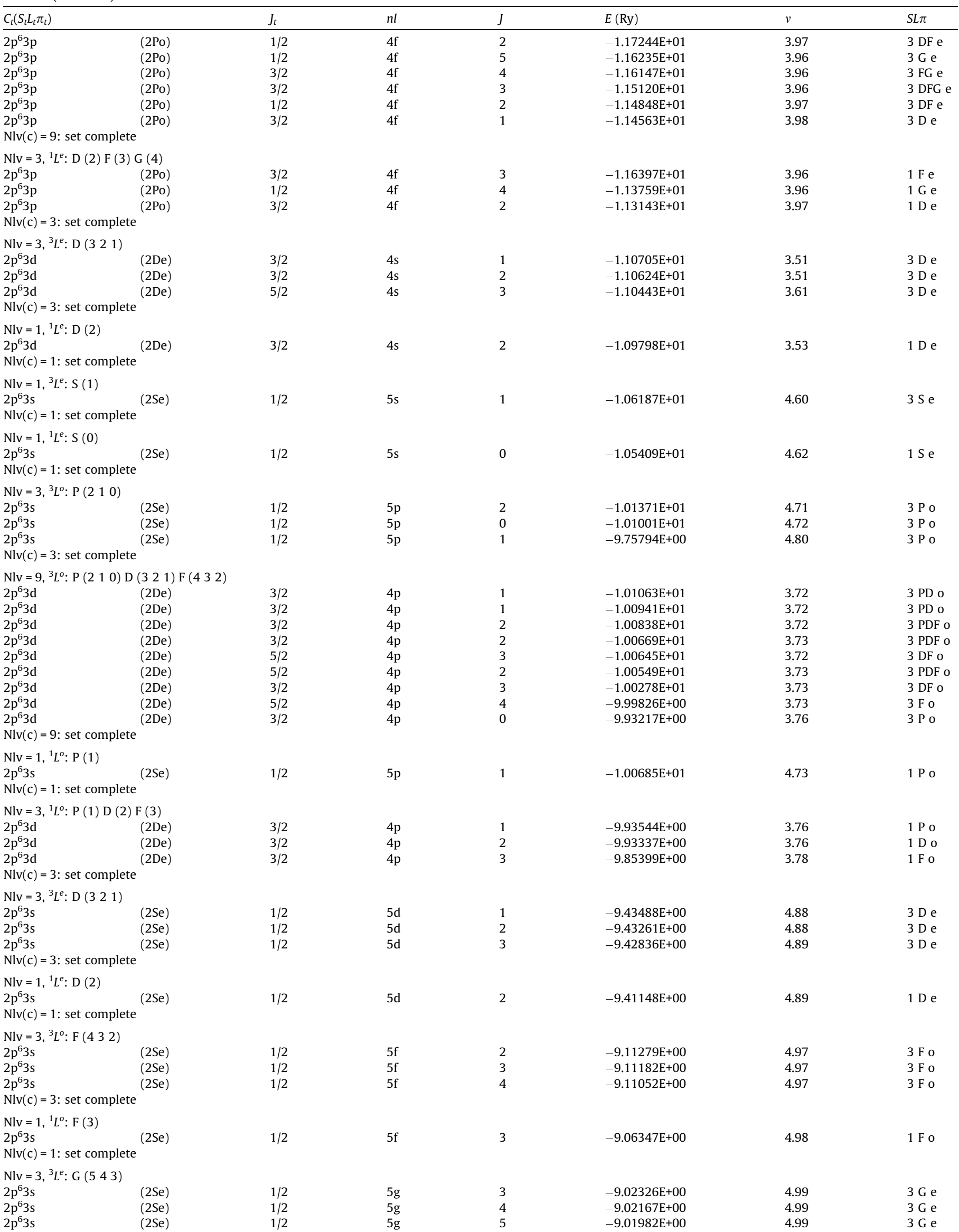


Table 1. (continued)

\begin{tabular}{|c|c|c|c|c|c|c|c|}
\hline$C_{t}\left(S_{t} L_{t} \pi_{t}\right)$ & & $J_{t}$ & $n l$ & $J$ & $E(\mathrm{Ry})$ & $v$ & $S L \pi$ \\
\hline \multicolumn{8}{|c|}{$\operatorname{Nlv}(\mathrm{c})=3$ : set complete } \\
\hline $\begin{array}{l}\operatorname{Nlv}=1, \\
2 p^{6} 3 s \\
\operatorname{Nlv}(c)=\end{array}$ & (2Se) & $1 / 2$ & $5 g$ & 4 & $-8.99516 \mathrm{E}+00$ & 5.00 & $1 \mathrm{G} \mathrm{e}$ \\
\hline \multicolumn{8}{|c|}{$\mathrm{Nlv}=13,{ }^{3} L^{e}: \mathrm{S}\left(\begin{array}{l}1\end{array}\right) \mathrm{P}\left(\begin{array}{lll}2 & 1 & 0\end{array}\right) \mathrm{D}\left(\begin{array}{lll}3 & 2 & 1\end{array}\right) \mathrm{F}\left(\begin{array}{lll}4 & 3 & 2\end{array}\right) \mathrm{G}\left(\begin{array}{lll}5 & 4 & 3\end{array}\right)$} \\
\hline $2 p^{6} 3 d$ & (2De) & $3 / 2$ & $4 d$ & 3 & $-8.87292 \mathrm{E}+00$ & 4.04 & 3 DFG e \\
\hline $2 p^{6} 3 d$ & (2De) & $3 / 2$ & $4 d$ & 1 & $-8.83134 \mathrm{E}+00$ & 4.05 & 3 SPD e \\
\hline $2 p^{6} 3 d$ & (2De) & $3 / 2$ & $4 d$ & 2 & $-8.81923 \mathrm{E}+00$ & 4.05 & 3 PDF e \\
\hline $2 p^{6} 3 d$ & (2De) & $3 / 2$ & $4 d$ & 3 & $-8.80569 \mathrm{E}+00$ & 4.05 & 3 DFG e \\
\hline $2 p^{6} 3 d$ & (2De) & $5 / 2$ & $4 d$ & 3 & $-8.79167 \mathrm{E}+00$ & 5.06 & 3 DFG e \\
\hline $2 p^{6} 3 d$ & (2De) & $3 / 2$ & $4 d$ & 1 & $-8.77878 \mathrm{E}+00$ & 4.06 & 3 SPD e \\
\hline $2 p^{6} 3 d$ & (2De) & $3 / 2$ & $4 d$ & 4 & $-8.77795 \mathrm{E}+00$ & 4.06 & 3 FG e \\
\hline $2 p^{6} 3 d$ & (2De) & $5 / 2$ & $4 d$ & 5 & $-8.75900 \mathrm{E}+00$ & 4.07 & $3 \mathrm{Ge}$ \\
\hline $2 p^{6} 3 d$ & (2De) & $3 / 2$ & $4 d$ & 1 & $-8.67371 \mathrm{E}+00$ & 4.09 & 3 SPD e \\
\hline $2 p^{6} 3 d$ & (2De) & $5 / 2$ & $4 d$ & 2 & $-8.62585 E+00$ & 4.11 & 3 PDF e \\
\hline $2 p^{6} 3 d$ & (2De) & $5 / 2$ & $4 d$ & 4 & $-8.59901 \mathrm{E}+00$ & 3.90 & 3 FG e \\
\hline $2 p^{6} 3 d$ & (2De) & $3 / 2$ & $4 d$ & 2 & $-8.48381 \mathrm{E}+00$ & 4.15 & 3 PDF e \\
\hline $2 p^{6} 3 d$ & (2De) & $3 / 2$ & $4 d$ & 0 & $-8.45982 \mathrm{E}+00$ & 4.00 & $3 \mathrm{Pe}$ \\
\hline \multicolumn{8}{|c|}{$\operatorname{Nlv}(c)=13:$ set complete } \\
\hline \multicolumn{8}{|c|}{$\mathrm{Nlv}=5,{ }^{1} L^{e}: \mathrm{S}(0) \mathrm{P}(1) \mathrm{D}(2) \mathrm{F}(3) \mathrm{G}(4)$} \\
\hline $2 p^{6} 3 d$ & (2De) & $3 / 2$ & $4 d$ & 3 & $-8.61347 \mathrm{E}+00$ & 3.90 & $1 \mathrm{~F} \mathrm{e}$ \\
\hline $2 p^{6} 3 d$ & (2De) & $3 / 2$ & $4 d$ & 1 & $-8.45410 \mathrm{E}+00$ & 4.00 & $1 \mathrm{P} \mathrm{e}$ \\
\hline $2 p^{6} 3 d$ & (2De) & $5 / 2$ & $4 d$ & 2 & $-8.44292 \mathrm{E}+00$ & 4.16 & $1 \mathrm{D} \mathrm{e}$ \\
\hline $2 p^{6} 3 d$ & (2De) & $5 / 2$ & $4 d$ & 4 & $-8.41900 \mathrm{E}+00$ & 4.17 & $1 \mathrm{G} \mathrm{e}$ \\
\hline $2 p^{6} 3 d$ & (2De) & $3 / 2$ & $4 d$ & 0 & $-8.16289 \mathrm{E}+00$ & 4.00 & $1 \mathrm{~S} \mathrm{e}$ \\
\hline \multicolumn{8}{|c|}{$\mathrm{Nlv}(\mathrm{c})=5$ : set complete } \\
\hline \multicolumn{8}{|c|}{$\mathrm{Nlv}=9,{ }^{3} L^{o}: \mathrm{P}\left(\begin{array}{lll}2 & 1 & 0\end{array}\right) \mathrm{D}\left(\begin{array}{lll}3 & 2 & 1\end{array}\right) \mathrm{F}\left(\begin{array}{lll}4 & 3 & 2\end{array}\right)$} \\
\hline $2 p^{6} 3 p$ & (2Po) & $1 / 2$ & $5 d$ & 4 & $-8.26285 \mathrm{E}+00$ & 4.57 & $3 \mathrm{Fo}$ \\
\hline $2 p^{6} 3 p$ & (2Po) & $1 / 2$ & $5 d$ & 3 & $-8.16889 E+00$ & 4.95 & $3 \mathrm{DF} o$ \\
\hline $2 p^{6} 3 p$ & (2Po) & $3 / 2$ & $5 d$ & 2 & $-8.07410 \mathrm{E}+00$ & 4.88 & 3 PDF o \\
\hline $2 p^{6} 3 p$ & (2Po) & $1 / 2$ & $5 d$ & 3 & $-7.98156 \mathrm{E}+00$ & 4.88 & $3 \mathrm{DF} o$ \\
\hline $2 p^{6} 3 p$ & (2Po) & $3 / 2$ & $5 d$ & 2 & $-7.93125 \mathrm{E}+00$ & 4.60 & 3 PDF o \\
\hline $2 p^{6} 3 p$ & (2Po) & $3 / 2$ & $5 d$ & 2 & $-7.91568 \mathrm{E}+00$ & 4.60 & 3 PDF o \\
\hline $2 p^{6} 3 p$ & (2Po) & $1 / 2$ & $5 d$ & 1 & $-7.88490 \mathrm{E}+00$ & 4.88 & 3 PD o \\
\hline $2 p^{6} 3 p$ & (2Po) & $3 / 2$ & $5 d$ & 1 & $-7.87187 \mathrm{E}+00$ & 4.88 & 3 PD o \\
\hline $2 p^{6} 3 p$ & (2Po) & $1 / 2$ & $5 d$ & 0 & $-7.86951 \mathrm{E}+00$ & 4.88 & $3 \mathrm{Po}$ \\
\hline \multicolumn{8}{|c|}{$\operatorname{Nlv}(c)=9:$ set complete } \\
\hline \multicolumn{8}{|c|}{ 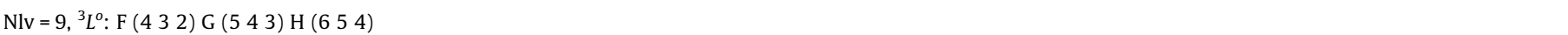 } \\
\hline $2 p^{6} 3 p$ & (2Po) & $3 / 2$ & $5 g$ & 4 & $-8.24679 \mathrm{E}+00$ & 5.00 & 3 FGH o \\
\hline $2 p^{6} 3 p$ & (2Po) & $1 / 2$ & $5 g$ & 5 & $-8.24598 \mathrm{E}+00$ & 4.57 & $3 \mathrm{GH}$ o \\
\hline $2 p^{6} 3 p$ & (2Po) & $3 / 2$ & $5 \mathrm{~g}$ & 6 & $-8.23032 \mathrm{E}+00$ & 4.53 & $3 \mathrm{H} \mathrm{o}$ \\
\hline $2 p^{6} 3 p$ & (2Po) & $1 / 2$ & $5 \mathrm{~g}$ & 4 & $-8.16120 \mathrm{E}+00$ & 4.88 & 3 FGH o \\
\hline $2 p^{6} 3 p$ & (2Po) & $1 / 2$ & $5 \mathrm{~g}$ & 4 & $-7.97148 \mathrm{E}+00$ & 4.88 & 3 FGH o \\
\hline $2 p^{6} 3 p$ & (2Po) & $3 / 2$ & $5 \mathrm{~g}$ & 5 & $-7.96131 \mathrm{E}+00$ & 4.59 & $3 \mathrm{GH}$ o \\
\hline $2 p^{6} 3 p$ & (2Po) & $3 / 2$ & $5 \mathrm{~g}$ & 3 & $-7.91413 \mathrm{E}+00$ & 4.88 & 3 FG o \\
\hline $2 p^{6} 3 p$ & (2Po) & $3 / 2$ & $5 \mathrm{~g}$ & 3 & $-7.87763 \mathrm{E}+00$ & 4.88 & 3 FG o \\
\hline $2 p^{6} 3 p$ & (2Po) & $3 / 2$ & $5 \mathrm{~g}$ & 2 & $-7.87644 \mathrm{E}+00$ & 4.98 & $3 \mathrm{Fo}$ \\
\hline \multicolumn{8}{|c|}{$\operatorname{Nlv}(c)=9$ : set complete } \\
\hline
\end{tabular}


Table 2

E1 transition probabilities for observed levels of Fe XV, grouped as fine structure components of $L S$ multiplets. See page 583 for Explanation of Tables.

\begin{tabular}{|c|c|c|c|c|c|c|}
\hline$C_{i}-C_{k}$ & $T_{i}-T_{k}$ & $g_{i}: \mathrm{I}-g_{j}: \mathrm{K}$ & $E_{i k}(\AA)$ & $f$ & $S$ & $A\left(\mathrm{~s}^{-1}\right)$ \\
\hline $2 p^{6} 3 s^{2}-3 s 3 p$ & $1 \mathrm{Se}-3 \mathrm{Po}$ & $1: 1-3: 1$ & 417.27 & $3.26 \mathrm{E}-03$ & $4.47 \mathrm{E}-03$ & $4.16 E+07$ \\
\hline $2 p^{6} 3 s^{2}-3 s 3 p$ & $1 \mathrm{Se}-1 \mathrm{Po}$ & $1: 1-3: 2$ & 284.17 & $7.90 \mathrm{E}-01$ & $7.39 \mathrm{E}-01$ & $2.18 \mathrm{E}+10$ \\
\hline $2 p^{6} 3 s^{2}-3 p 3 d$ & 1Se-3Do & $1: 1-3: 3$ & 101.74 & $9.20 \mathrm{E}-07$ & $3.08 \mathrm{E}-07$ & $1.97 \mathrm{E}+05$ \\
\hline $2 p^{6} 3 s^{2}-3 p 3 d$ & $1 \mathrm{Se}-3 \mathrm{Po}$ & $1: 1-3: 4$ & 100.38 & $6.25 \mathrm{E}-08$ & $2.07 \mathrm{E}-08$ & $1.38 \mathrm{E}+04$ \\
\hline $2 p^{6} 3 s^{2}-3 p 3 d$ & $1 \mathrm{Se}-1 \mathrm{Po}$ & $1: 1-3: 5$ & 93.03 & $1.26 \mathrm{E}-03$ & $3.87 \mathrm{E}-04$ & $3.25 E+08$ \\
\hline $2 p^{6} 3 s^{2}-3 s 4 p$ & 1Se-1Po & $1: 1-3: 7$ & 52.91 & $2.87 \mathrm{E}-01$ & $5.00 \mathrm{E}-02$ & $2.28 \mathrm{E}+11$ \\
\hline $2 p^{6} 3 s^{2}-3 s 5 p$ & $1 \mathrm{Se}-1 \mathrm{Po}$ & $1: 1-3: 15$ & 38.96 & $6.11 \mathrm{E}-02$ & $7.84 \mathrm{E}-03$ & $8.95 E+10$ \\
\hline $3 s 3 p-3 p^{2}$ & $3 \mathrm{Po}-3 \mathrm{Pe}$ & $3: 1-1: 2$ & 317.59 & $8.74 \mathrm{E}-02$ & $2.74 \mathrm{E}-01$ & $1.73 E+10$ \\
\hline $3 s 3 p-3 p^{2}$ & 3Рo-3Pe & $1: 1-3: 1$ & 302.33 & $2.79 \mathrm{E}-01$ & $2.77 \mathrm{E}-01$ & $6.78 \mathrm{E}+09$ \\
\hline $3 s 3 p-3 p^{2}$ & $3 \mathrm{Po}-3 \mathrm{Pe}$ & $3: 1-3: 1$ & 307.75 & $6.78 \mathrm{E}-02$ & $2.06 \mathrm{E}-01$ & $4.78 \mathrm{E}+09$ \\
\hline $3 s 3 p-3 p^{2}$ & $3 \mathrm{Po}-3 \mathrm{Pe}$ & $5: 1-3: 1$ & 321.78 & $6.48 \mathrm{E}-02$ & $3.43 \mathrm{E}-01$ & $6.96 \mathrm{E}+09$ \\
\hline $3 \mathrm{~s} 3 \mathrm{p}-3 \mathrm{p}^{2}$ & $3 \mathrm{Po}-3 \mathrm{Pe}$ & $3: 1-5: 2$ & 292.27 & $9.15 \mathrm{E}-02$ & $2.64 \mathrm{E}-01$ & $4.29 \mathrm{E}+09$ \\
\hline $3 s 3 p-3 p^{2}$ & $3 \mathrm{Po}-3 \mathrm{Pe}$ & $5: 1-5: 2$ & 304.89 & $1.71 \mathrm{E}-01$ & $8.60 \mathrm{E}-01$ & $1.23 \mathrm{E}+10$ \\
\hline$L S$ & $3 \mathrm{Po}-3 \mathrm{Pe}$ & $9-9$ & & $2.44 \mathrm{E}-01$ & $2.22 \mathrm{E}+00$ & $1.72 \mathrm{E}+10$ \\
\hline $3 s 3 p-3 p^{2}$ & $1 \mathrm{Po}-3 \mathrm{Pe}$ & $3: 2-1: 2$ & 493.54 & $6.82 \mathrm{E}-04$ & $3.32 \mathrm{E}-03$ & $5.60 \mathrm{E}+07$ \\
\hline $3 s 3 p-3 p^{2}$ & $1 \mathrm{Po}-3 \mathrm{Pe}$ & $3: 2-3: 1$ & 470.16 & $2.78 \mathrm{E}-04$ & $1.29 \mathrm{E}-03$ & $8.39 \mathrm{E}+06$ \\
\hline $3 s 3 p-3 p^{2}$ & 1Po-3Pe & $3: 2-5: 2$ & 434.97 & $2.40 \mathrm{E}-02$ & $1.03 \mathrm{E}-01$ & $5.07 \mathrm{E}+08$ \\
\hline $3 p^{2}-3 p 3 d$ & 3Pe-3Do & $1: 2-3: 3$ & 233.45 & $6.19 \mathrm{E}-01$ & $4.76 \mathrm{E}-01$ & $2.52 \mathrm{E}+10$ \\
\hline $3 p^{2}-3 p 3 d$ & 3Pe-3Do & $3: 1-3: 3$ & 239.08 & $3.94 \mathrm{E}-02$ & $9.29 \mathrm{E}-02$ & $4.59 \mathrm{E}+09$ \\
\hline $3 p^{2}-3 p 3 d$ & 3Pe-3Do & $3: 1-5: 4$ & 231.47 & $3.40 \mathrm{E}-01$ & $7.77 \mathrm{E}-01$ & $2.54 \mathrm{E}+10$ \\
\hline $3 p^{2}-3 p 3 d$ & 3Pe-3Do & $5: 2-3: 3$ & 249.33 & $1.20 \mathrm{E}-03$ & $4.93 \mathrm{E}-03$ & $2.15 \mathrm{E}+08$ \\
\hline $3 p^{2}-3 p 3 d$ & 3Pe-3Do & $5: 2-5: 4$ & 241.07 & $1.05 \mathrm{E}-02$ & $4.16 \mathrm{E}-02$ & $1.20 \mathrm{E}+09$ \\
\hline $3 p^{2}-3 p 3 d$ & 3Pe-3Do & $5: 2-7: 2$ & 242.10 & $2.90 \mathrm{E}-01$ & $1.16 \mathrm{E}+00$ & $2.36 \mathrm{E}+10$ \\
\hline$L S$ & 3Pe-3Do & $9-15$ & & $3.63 \mathrm{E}-01$ & $2.55 \mathrm{E}+00$ & $2.58 \mathrm{E}+10$ \\
\hline $3 p^{2}-3 p 3 d$ & $3 \mathrm{Pe}-3 \mathrm{Po}$ & $1: 2-3: 4$ & 226.39 & $1.32 \mathrm{E}-03$ & $9.81 \mathrm{E}-04$ & $5.71 \mathrm{E}+07$ \\
\hline $3 p^{2}-3 p 3 d$ & 3Pe-3Po & $3: 1-1: 2$ & 231.86 & $5.47 \mathrm{E}-02$ & $1.25 \mathrm{E}-01$ & $2.04 \mathrm{E}+10$ \\
\hline $3 p^{2}-3 p 3 d$ & $3 \mathrm{Pe}-3 \mathrm{Po}$ & $3: 1-3: 4$ & 231.67 & $1.15 \mathrm{E}-01$ & $2.64 \mathrm{E}-01$ & $1.43 \mathrm{E}+10$ \\
\hline $3 p^{2}-3 p 3 d$ & $3 \mathrm{Pe}-3 \mathrm{Po}$ & $3: 1-5: 5$ & 238.71 & $6.32 \mathrm{E}-02$ & $1.49 \mathrm{E}-01$ & $4.44 \mathrm{E}+09$ \\
\hline $3 p^{2}-3 p 3 d$ & 3Pe-3Po & $5: 2-3: 4$ & 241.29 & $3.56 \mathrm{E}-02$ & $1.42 \mathrm{E}-01$ & $6.80 \mathrm{E}+09$ \\
\hline $3 p^{2}-3 p 3 d$ & 3Pe-3Po & $5: 2-5: 5$ & 248.94 & $1.51 \mathrm{E}-01$ & $6.21 \mathrm{E}-01$ & $1.63 \mathrm{E}+10$ \\
\hline$L S$ & $3 \mathrm{Pe}-3 \mathrm{Po}$ & $9-9$ & & $1.81 \mathrm{E}-01$ & $1.30 \mathrm{E}+00$ & $2.06 \mathrm{E}+10$ \\
\hline $3 p^{2}-3 p 3 d$ & $3 \mathrm{Pe}-1 \mathrm{Po}$ & $1: 2-3: 5$ & 192.17 & $3.50 \mathrm{E}-03$ & $2.21 \mathrm{E}-03$ & $2.10 \mathrm{E}+08$ \\
\hline $3 p^{2}-3 p 3 d$ & $3 \mathrm{Pe}-1 \mathrm{Po}$ & $3: 1-3: 5$ & 195.97 & $4.29 \mathrm{E}-04$ & $8.30 \mathrm{E}-04$ & $7.44 \mathrm{E}+07$ \\
\hline $3 p^{2}-3 p 3 d$ & $3 \mathrm{Pe}-1 \mathrm{Po}$ & $5: 2-3: 5$ & 202.81 & $3.37 \mathrm{E}-04$ & $1.13 \mathrm{E}-03$ & $9.12 \mathrm{E}+07$ \\
\hline $3 p^{2}-3 s 4 p$ & $3 \mathrm{Pe}-1 \mathrm{Po}$ & $1: 2-3: 7$ & 74.88 & $5.10 \mathrm{E}-05$ & $1.26 \mathrm{E}-05$ & $2.02 \mathrm{E}+07$ \\
\hline $3 p^{2}-3 s 4 p$ & $3 \mathrm{Pe}-1 \mathrm{Po}$ & $3: 1-3: 7$ & 75.45 & $5.52 \mathrm{E}-06$ & $4.12 \mathrm{E}-06$ & $6.47 \mathrm{E}+06$ \\
\hline $3 p^{2}-3 s 4 p$ & $3 \mathrm{Pe}-1 \mathrm{Po}$ & $5: 2-3: 7$ & 76.44 & $3.66 \mathrm{E}-03$ & $4.61 \mathrm{E}-03$ & $6.97 \mathrm{E}+09$ \\
\hline $3 p^{2}-3 s 5 p$ & $3 \mathrm{Pe}-1 \mathrm{Po}$ & $1: 2-3: 15$ & 49.69 & $2.50 \mathrm{E}-04$ & $4.09 \mathrm{E}-05$ & $2.25 \mathrm{E}+08$ \\
\hline $3 p^{2}-3 s 5 p$ & $3 \mathrm{Pe}-1 \mathrm{Po}$ & $3: 1-3: 15$ & 49.94 & $1.09 \mathrm{E}-04$ & $5.35 \mathrm{E}-05$ & $2.90 \mathrm{E}+08$ \\
\hline $3 p^{2}-3 s 5 p$ & $3 \mathrm{Pe}-1 \mathrm{Po}$ & $5: 2-3: 15$ & 50.37 & $2.38 \mathrm{E}-06$ & $1.97 \mathrm{E}-06$ & $1.04 \mathrm{E}+07$ \\
\hline $3 s 3 p-3 p^{2}$ & $3 \mathrm{Po}-1 \mathrm{Se}$ & $3: 1-1: 3$ & 238.11 & $8.07 \mathrm{E}-04$ & $1.90 \mathrm{E}-03$ & $2.85 \mathrm{E}+08$ \\
\hline $3 \mathrm{~s} 3 \mathrm{p}-3 \mathrm{p}^{2}$ & 1Po-1Se & $3: 2-1: 3$ & 324.97 & $1.03 \mathrm{E}-01$ & $3.29 \mathrm{E}-01$ & $1.94 \mathrm{E}+10$ \\
\hline $3 p^{2}-3 p 3 d$ & 1Se-3Do & $1: 3-3: 3$ & 309.37 & $1.49 \mathrm{E}-03$ & $1.51 \mathrm{E}-03$ & $3.45 \mathrm{E}+07$ \\
\hline $3 p^{2}-3 p 3 d$ & $1 \mathrm{Se}-3 \mathrm{Po}$ & $1: 3-3: 4$ & 297.08 & $4.11 \mathrm{E}-09$ & $4.02 \mathrm{E}-09$ & $1.04 \mathrm{E}+02$ \\
\hline $3 p^{2}-3 p 3 d$ & 1Se-1Po & $1: 3-3: 5$ & 240.81 & $6.43 \mathrm{E}-01$ & $5.10 \mathrm{E}-01$ & $2.47 E+10$ \\
\hline $3 p^{2}-3 s 4 p$ & $1 \mathrm{Se}-1 \mathrm{Po}$ & $1: 3-3: 7$ & 81.28 & $1.61 \mathrm{E}-03$ & $4.29 \mathrm{E}-04$ & $5.40 \mathrm{E}+08$ \\
\hline $3 p^{2}-3 s 5 p$ & $1 \mathrm{Se}-1 \mathrm{Po}$ & $1: 3-3: 15$ & 52.43 & $1.10 \mathrm{E}-03$ & $1.90 \mathrm{E}-04$ & $8.92 \mathrm{E}+08$ \\
\hline $3 s 3 p-3 d^{2}$ & $3 \mathrm{Po}-1 \mathrm{Se}$ & $3: 1-1: 5$ & 80.17 & $2.06 \mathrm{E}-06$ & $1.63 \mathrm{E}-06$ & $6.43 E+06$ \\
\hline $3 s 3 p-3 d^{2}$ & 1Po-1Se & $3: 2-1: 5$ & 88.09 & $7.27 \mathrm{E}-04$ & $6.32 \mathrm{E}-04$ & $1.87 \mathrm{E}+09$ \\
\hline $3 p 3 d-3 d^{2}$ & 3Do-1Se & $3: 3-1: 5$ & 198.34 & $1.32 \mathrm{E}-03$ & $2.59 \mathrm{E}-03$ & $6.71 E+08$ \\
\hline $3 p 3 d-3 d^{2}$ & $3 \mathrm{Po}-1 \mathrm{Se}$ & $3: 4-1: 5$ & 203.74 & $8.05 \mathrm{E}-06$ & $1.62 \mathrm{E}-05$ & $3.88 \mathrm{E}+06$ \\
\hline $3 p 3 d-3 d^{2}$ & $1 \mathrm{Po}-1 \mathrm{Se}$ & $3: 5-1: 5$ & 242.62 & $1.65 \mathrm{E}-01$ & $3.95 \mathrm{E}-01$ & $5.60 \mathrm{E}+10$ \\
\hline $3 d^{2}-3 s 4 p$ & 1Se-1Po & $1: 5-3: 7$ & 248.19 & $1.99 \mathrm{E}-03$ & $1.62 \mathrm{E}-03$ & $7.17 \mathrm{E}+07$ \\
\hline $3 d^{2}-3 s 5 p$ & $1 \mathrm{Se}-1 \mathrm{Po}$ & $1: 5-3: 15$ & 92.60 & $6.84 \mathrm{E}-03$ & $2.08 \mathrm{E}-03$ & $1.77 \mathrm{E}+09$ \\
\hline $3 s 3 p-3 s 4 s$ & $3 \mathrm{Po}-1 \mathrm{Se}$ & $3: 1-1: 6$ & 64.63 & $2.13 \mathrm{E}-04$ & $1.36 \mathrm{E}-04$ & $1.02 \mathrm{E}+09$ \\
\hline $3 s 3 p-3 s 4 s$ & 1Po-1Se & $3: 2-1: 6$ & 69.68 & $5.31 \mathrm{E}-02$ & $3.65 \mathrm{E}-02$ & $2.19 \mathrm{E}+11$ \\
\hline $3 p 3 d-3 s 4 s$ & 3Do-1Se & $3: 3-1: 6$ & 124.36 & $1.48 \mathrm{E}-07$ & $1.82 \mathrm{E}-07$ & $1.92 \mathrm{E}+05$ \\
\hline $3 p 3 d-3 s 4 s$ & $3 \mathrm{Po}-1 \mathrm{Se}$ & $3: 4-1: 6$ & 126.46 & $2.02 \mathrm{E}-09$ & $2.53 \mathrm{E}-09$ & $2.53 \mathrm{E}+03$ \\
\hline $3 p 3 d-3 s 4 s$ & 1Po-1Se & $3: 5-1: 6$ & 140.43 & $5.48 \mathrm{E}-08$ & $7.60 \mathrm{E}-08$ & $5.56 \mathrm{E}+04$ \\
\hline $3 s 4 s-3 s 4 p$ & 1Se-1Po & $1: 6-3: 7$ & 971.09 & $2.70 \mathrm{E}-01$ & $8.64 \mathrm{E}-01$ & $6.37 \mathrm{E}+08$ \\
\hline $3 s 4 s-3 s 5 p$ & $1 \mathrm{Se}-1 \mathrm{Po}$ & $1: 6-3: 15$ & 128.20 & $9.47 \mathrm{E}-02$ & $4.00 \mathrm{E}-02$ & $1.28 \mathrm{E}+10$ \\
\hline $3 s 3 p-3 s 3 d$ & 3Po-3De & $1: 1-3: 2$ & 224.75 & $3.07 \mathrm{E}-01$ & $2.27 \mathrm{E}-01$ & $1.35 \mathrm{E}+10$ \\
\hline $3 s 3 p-3 s 3 d$ & 3Po-3De & $3: 1-3: 2$ & 227.73 & $7.53 \mathrm{E}-02$ & $1.69 \mathrm{E}-01$ & $9.68 \mathrm{E}+09$ \\
\hline $3 s 3 p-3 s 3 d$ & 3Po-3De & $5: 1-3: 2$ & 235.32 & $2.94 \mathrm{E}-03$ & $1.14 \mathrm{E}-02$ & $5.91 \mathrm{E}+08$ \\
\hline $3 s 3 p-3 s 3 d$ & 3Po-3De & $3: 1-5: 3$ & 227.20 & $2.27 \mathrm{E}-01$ & $5.09 \mathrm{E}-01$ & $1.76 \mathrm{E}+10$ \\
\hline $3 \mathrm{~s} 3 \mathrm{p}-3 \mathrm{~s} 3 \mathrm{~d}$ & 3Po-3De & $5: 1-5: 3$ & 234.76 & $4.44 \mathrm{E}-02$ & $1.72 \mathrm{E}-01$ & $5.37 \mathrm{E}+09$ \\
\hline $3 s 3 p-3 s 3 d$ & 3Po-3De & $5: 1-7: 1$ & 233.87 & $2.48 \mathrm{E}-01$ & $9.55 \mathrm{E}-01$ & $2.16 \mathrm{E}+10$ \\
\hline$L S$ & 3Po-3De & $9-15$ & & $2.99 \mathrm{E}-01$ & $2.04 \mathrm{E}+00$ & $2.25 \mathrm{E}+10$ \\
\hline $3 s 3 p-3 s 4 s$ & $3 \mathrm{Po}-3 \mathrm{Se}$ & $1: 1-3: 4$ & 65.37 & $7.29 \mathrm{E}-02$ & $1.57 \mathrm{E}-02$ & $3.79 \mathrm{E}+10$ \\
\hline $3 s 3 p-3 s 4 s$ & $3 \mathrm{Po}-3 \mathrm{Se}$ & $3: 1-3: 4$ & 65.61 & $7.36 \mathrm{E}-02$ & $4.77 \mathrm{E}-02$ & $1.14 \mathrm{E}+11$ \\
\hline $3 s 3 p-3 s 4 s$ & $3 \mathrm{Po}-3 \mathrm{Se}$ & $5: 1-3: 4$ & 66.23 & $7.70 \mathrm{E}-02$ & $8.39 \mathrm{E}-02$ & $1.95 \mathrm{E}+11$ \\
\hline$L S$ & $3 \mathrm{Po}-3 \mathrm{Se}$ & $9-3$ & & $7.54 \mathrm{E}-02$ & $1.47 \mathrm{E}-01$ & $3.47 \mathrm{E}+11$ \\
\hline $3 s 3 p-3 s 4 d$ & 3Po-3De & $1: 1-3: 5$ & 55.63 & $3.42 \mathrm{E}-01$ & $6.27 \mathrm{E}-02$ & $2.46 \mathrm{E}+11$ \\
\hline $3 s 3 p-3 s 4 d$ & 3Po-3De & $3: 1-3: 5$ & 55.81 & $8.59 \mathrm{E}-02$ & $4.73 \mathrm{E}-02$ & $1.84 \mathrm{E}+11$ \\
\hline $3 s 3 p-3 s 4 d$ & 3Po-3De & $5: 1-3: 5$ & 56.26 & $3.51 \mathrm{E}-03$ & $3.25 \mathrm{E}-03$ & $1.23 \mathrm{E}+10$ \\
\hline
\end{tabular}


Table 2. (continued)

\begin{tabular}{|c|c|c|c|c|c|c|}
\hline$C_{i}-C_{k}$ & $T_{i}-T_{k}$ & $g_{i}: \mathrm{I}-g_{j}: \mathrm{K}$ & $E_{i k}(\AA)$ & $f$ & $S$ & $A\left(\mathrm{~s}^{-1}\right)$ \\
\hline $3 s 3 p-3 s 4 d$ & 3Po-3De & $3: 1-5: 8$ & 55.79 & $2.52 \mathrm{E}-01$ & $1.39 \mathrm{E}-01$ & $3.24 \mathrm{E}+11$ \\
\hline $3 s 3 p-3 s 4 d$ & 3Po-3De & $5: 1-5: 8$ & 56.24 & $5.07 \mathrm{E}-02$ & $4.70 \mathrm{E}-02$ & $1.07 \mathrm{E}+11$ \\
\hline $3 s 3 p-3 s 4 d$ & 3Po-3De & $5: 1-7: 3$ & 56.20 & $2.87 \mathrm{E}-01$ & $2.66 \mathrm{E}-01$ & $4.33 \mathrm{E}+11$ \\
\hline$L S$ & 3Po-3De & $9-15$ & & $3.40 \mathrm{E}-01$ & $5.65 \mathrm{E}-01$ & $4.33 \mathrm{E}+11$ \\
\hline $3 s 3 p-3 p 4 f$ & 3Po-3De & $1: 1-3: 10$ & 45.74 & $1.03 \mathrm{E}-03$ & $1.55 \mathrm{E}-04$ & $1.09 \mathrm{E}+09$ \\
\hline $3 s 3 p-3 p 4 f$ & 3Po-3De & $3: 1-3: 10$ & 45.86 & $3.07 \mathrm{E}-04$ & $1.39 \mathrm{E}-04$ & $9.74 \mathrm{E}+08$ \\
\hline $3 s 3 p-3 p 4 f$ & 3Po-3De & $5: 1-3: 10$ & 46.16 & $1.67 \mathrm{E}-05$ & $1.27 \mathrm{E}-05$ & $8.71 E+07$ \\
\hline $3 s 3 p-3 p 4 f$ & 3Po-3De & $3: 1-5: 14$ & 45.98 & $6.69 \mathrm{E}-04$ & $3.04 \mathrm{E}-04$ & $1.27 \mathrm{E}+09$ \\
\hline $3 s 3 p-3 p 4 f$ & 3Po-3De & $5: 1-5: 14$ & 46.29 & $1.87 \mathrm{E}-04$ & $1.43 \mathrm{E}-04$ & $5.82 \mathrm{E}+08$ \\
\hline $3 s 3 p-3 p 4 f$ & 3Po-3De & $5: 1-7: 8$ & 46.31 & $6.52 \mathrm{E}-04$ & $4.97 \mathrm{E}-04$ & $1.45 \mathrm{E}+09$ \\
\hline$L S$ & 3Po-3De & $9-15$ & & $9.15 \mathrm{E}-04$ & $1.25 \mathrm{E}-03$ & $1.72 E+09$ \\
\hline $3 s 3 p-3 s 5 s$ & $3 \mathrm{Po}-3 \mathrm{Se}$ & $1: 1-3: 12$ & 43.27 & $1.35 \mathrm{E}-02$ & $1.92 \mathrm{E}-03$ & $1.60 \mathrm{E}+10$ \\
\hline $3 s 3 p-3 s 5 s$ & $3 \mathrm{Po}-3 \mathrm{Se}$ & $3: 1-3: 12$ & 43.38 & $1.35 \mathrm{E}-02$ & $5.80 \mathrm{E}-03$ & $4.79 E+10$ \\
\hline $3 s 3 p-3 s 5 s$ & 3Po-3Se & $5: 1-3: 12$ & 43.65 & $1.39 \mathrm{E}-02$ & $9.97 \mathrm{E}-03$ & $8.10 \mathrm{E}+10$ \\
\hline$L S$ & $3 \mathrm{Po}-3 \mathrm{Se}$ & $9-3$ & & $1.37 \mathrm{E}-02$ & $1.77 \mathrm{E}-02$ & $1.45 \mathrm{E}+11$ \\
\hline $3 s 3 p-3 s 5 d$ & 3Po-3De & $1: 1-3: 13$ & 41.56 & $9.51 \mathrm{E}-02$ & $1.30 \mathrm{E}-02$ & $1.22 \mathrm{E}+11$ \\
\hline $3 s 3 p-3 s 5 d$ & 3Po-3De & $3: 1-3: 13$ & 41.66 & $2.38 \mathrm{E}-02$ & $9.80 \mathrm{E}-03$ & $9.15 \mathrm{E}+10$ \\
\hline $3 s 3 p-3 s 5 d$ & $3 \mathrm{Po}-3 \mathrm{De}$ & $5: 1-3: 13$ & 41.91 & $9.77 \mathrm{E}-04$ & $6.74 \mathrm{E}-04$ & $6.18 \mathrm{E}+09$ \\
\hline $3 s 3 p-3 s 5 d$ & $3 \mathrm{Po}-3 \mathrm{De}$ & $3: 1-5: 18$ & 41.66 & $7.15 \mathrm{E}-02$ & $2.94 \mathrm{E}-02$ & $1.65 \mathrm{E}+11$ \\
\hline $3 s 3 p-3 s 5 d$ & 3Po-3De & $5: 1-5: 18$ & 41.91 & $1.44 \mathrm{E}-02$ & $9.93 \mathrm{E}-03$ & $5.47 \mathrm{E}+10$ \\
\hline $3 s 3 p-3 s 5 d$ & 3Po-3De & $5: 1-7: 10$ & 41.90 & $8.16 \mathrm{E}-02$ & $5.63 \mathrm{E}-02$ & $2.21 \mathrm{E}+11$ \\
\hline$L S$ & 3Po-3De & $9-15$ & & $9.62 \mathrm{E}-02$ & $1.19 \mathrm{E}-01$ & $2.21 \mathrm{E}+11$ \\
\hline $3 s 3 d-3 p 3 d$ & 3De-3Po & $3: 2-1: 2$ & 315.34 & $5.23 \mathrm{E}-02$ & $1.63 \mathrm{E}-01$ & $1.05 \mathrm{E}+10$ \\
\hline $3 s 3 d-3 p 3 d$ & 3De-3Po & $3: 2-3: 4$ & 314.99 & $1.09 \mathrm{E}-01$ & $3.38 \mathrm{E}-01$ & $7.30 \mathrm{E}+09$ \\
\hline $3 s 3 d-3 p 3 d$ & 3De-3Po & $3: 2-5: 5$ & 328.15 & $3.56 \mathrm{E}-02$ & $1.15 \mathrm{E}-01$ & $1.32 \mathrm{E}+09$ \\
\hline $3 s 3 d-3 p 3 d$ & 3De-3Po & $5: 3-3: 4$ & 316.01 & $2.70 \mathrm{E}-02$ & $1.40 \mathrm{E}-01$ & $3.01 E+09$ \\
\hline $3 s 3 d-3 p 3 d$ & 3De-3Po & $5: 3-5: 5$ & 329.25 & $1.03 \mathrm{E}-01$ & $5.59 \mathrm{E}-01$ & $6.34 \mathrm{E}+09$ \\
\hline $3 s 3 d-3 p 3 d$ & 3De-3Po & $7: 1-5: 5$ & 331.02 & $1.27 \mathrm{E}-02$ & $9.70 \mathrm{E}-02$ & $1.08 \mathrm{E}+09$ \\
\hline$L S$ & 3De-3Po & $15-9$ & & $8.86 \mathrm{E}-02$ & $1.41 \mathrm{E}+00$ & $9.47 E+09$ \\
\hline $3 p 3 d-3 s 4 s$ & $3 \mathrm{Po}-3 \mathrm{Se}$ & $1: 2-3: 4$ & 130.24 & $3.44 \mathrm{E}-05$ & $1.47 \mathrm{E}-05$ & $4.50 \mathrm{E}+06$ \\
\hline $3 p 3 d-3 s 4 s$ & $3 \mathrm{Po}-3 \mathrm{Se}$ & $3: 4-3: 4$ & 130.30 & $2.82 \mathrm{E}-05$ & $3.63 \mathrm{E}-05$ & $1.11 \mathrm{E}+07$ \\
\hline $3 p 3 d-3 s 4 s$ & $3 \mathrm{Po}-3 \mathrm{Se}$ & $5: 5-3: 4$ & 128.17 & $1.95 \mathrm{E}-05$ & $4.11 \mathrm{E}-05$ & $1.32 \mathrm{E}+07$ \\
\hline$L S$ & $3 \mathrm{Po}-3 \mathrm{Se}$ & $9-3$ & & $2.41 \mathrm{E}-05$ & $9.21 \mathrm{E}-05$ & $2.88 \mathrm{E}+07$ \\
\hline $3 p 3 d-3 s 4 d$ & $3 \mathrm{Po}-3 \mathrm{De}$ & $1: 2-3: 5$ & 96.58 & $1.19 \mathrm{E}-04$ & $3.78 \mathrm{E}-05$ & $2.83 \mathrm{E}+07$ \\
\hline $3 p 3 d-3 s 4 d$ & 3Po-3De & $3: 4-3: 5$ & 96.61 & $3.99 \mathrm{E}-05$ & $3.80 \mathrm{E}-05$ & $2.85 \mathrm{E}+07$ \\
\hline $3 p 3 d-3 s 4 d$ & 3Po-3De & $5: 5-3: 5$ & 95.44 & $1.61 \mathrm{E}-05$ & $2.53 \mathrm{E}-05$ & $1.96 \mathrm{E}+07$ \\
\hline $3 p 3 d-3 s 4 d$ & 3Po-3De & $3: 4-5: 8$ & 96.55 & $4.62 \mathrm{E}-05$ & $4.41 \mathrm{E}-05$ & $1.98 \mathrm{E}+07$ \\
\hline $3 p 3 d-3 s 4 d$ & $3 \mathrm{Po}-3 \mathrm{De}$ & $5: 5-5: 8$ & 95.37 & $1.82 \mathrm{E}-05$ & $2.86 \mathrm{E}-05$ & $1.34 \mathrm{E}+07$ \\
\hline $3 p 3 d-3 s 4 d$ & $3 \mathrm{Po}-3 \mathrm{De}$ & $5: 5-7: 3$ & 95.27 & $1.88 \mathrm{E}-05$ & $2.95 \mathrm{E}-05$ & $9.86 \mathrm{E}+06$ \\
\hline$L S$ & $3 \mathrm{Po}-3 \mathrm{De}$ & $9-15$ & & $7.14 \mathrm{E}-05$ & $2.03 \mathrm{E}-04$ & $3.10 \mathrm{E}+07$ \\
\hline $3 p 3 d-3 p 4 f$ & 3Po-3De & $1: 2-3: 10$ & 70.21 & $9.48 \mathrm{E}-01$ & $2.19 \mathrm{E}-01$ & $4.28 \mathrm{E}+11$ \\
\hline $3 p 3 d-3 p 4 f$ & $3 \mathrm{Po}-3 \mathrm{De}$ & $3: 4-3: 10$ & 70.23 & $3.21 \mathrm{E}-01$ & $2.23 \mathrm{E}-01$ & $4.35 \mathrm{E}+11$ \\
\hline $3 p 3 d-3 p 4 f$ & $3 \mathrm{Po}-3 \mathrm{De}$ & $5: 5-3: 10$ & 69.61 & $2.77 \mathrm{E}-02$ & $3.18 \mathrm{E}-02$ & $6.37 \mathrm{E}+10$ \\
\hline $3 p 3 d-3 p 4 f$ & 3Po-3De & $3: 4-5: 14$ & 70.52 & $5.61 \mathrm{E}-01$ & $3.91 \mathrm{E}-01$ & $4.52 \mathrm{E}+11$ \\
\hline $3 p 3 d-3 p 4 f$ & 3Po-3De & $5: 5-5: 14$ & 69.89 & $2.46 \mathrm{E}-01$ & $2.83 \mathrm{E}-01$ & $3.36 \mathrm{E}+11$ \\
\hline $3 p 3 d-3 p 4 f$ & $3 \mathrm{Po}-3 \mathrm{De}$ & $5: 5-7: 8$ & 69.95 & $4.79 \mathrm{E}-01$ & $5.51 \mathrm{E}-01$ & $4.66 \mathrm{E}+11$ \\
\hline$L S$ & 3Po-3De & $9-15$ & & $8.17 \mathrm{E}-01$ & $1.70 \mathrm{E}+00$ & $6.65 E+11$ \\
\hline $3 p 3 d-3 s 5 s$ & $3 \mathrm{Po}-3 \mathrm{Se}$ & $1: 2-3: 12$ & 64.56 & $1.94 \mathrm{E}-04$ & $4.12 \mathrm{E}-05$ & $1.03 \mathrm{E}+08$ \\
\hline $3 p 3 d-3 s 5 s$ & $3 \mathrm{Po}-3 \mathrm{Se}$ & $3: 4-3: 12$ & 64.58 & $1.52 \mathrm{E}-04$ & $9.68 \mathrm{E}-05$ & $2.43 \mathrm{E}+08$ \\
\hline $3 p 3 d-3 s 5 s$ & $3 \mathrm{Po}-3 \mathrm{Se}$ & $5: 5-3: 12$ & 64.05 & $9.02 \mathrm{E}-05$ & $9.51 \mathrm{E}-05$ & $2.44 \mathrm{E}+08$ \\
\hline$L S$ & $3 \mathrm{Po}-3 \mathrm{Se}$ & $9-3$ & & $1.22 \mathrm{E}-04$ & $2.33 \mathrm{E}-04$ & $5.92 \mathrm{E}+08$ \\
\hline $3 p 3 d-3 s 5 d$ & 3Po-3De & $1: 2-3: 13$ & 60.82 & $3.65 \mathrm{E}-03$ & $7.31 \mathrm{E}-04$ & $2.20 \mathrm{E}+09$ \\
\hline $3 p 3 d-3 s 5 d$ & 3Po-3De & $3: 4-3: 13$ & 60.83 & $6.90 \mathrm{E}-04$ & $4.15 \mathrm{E}-04$ & $1.24 \mathrm{E}+09$ \\
\hline $3 p 3 d-3 s 5 d$ & 3Po-3De & $5: 5-3: 13$ & 60.36 & $1.69 \mathrm{E}-05$ & $1.68 \mathrm{E}-05$ & $5.15 E+07$ \\
\hline $3 p 3 d-3 s 5 d$ & 3Po-3De & $3: 4-5: 18$ & 60.84 & $2.07 \mathrm{E}-03$ & $1.25 \mathrm{E}-03$ & $2.24 \mathrm{E}+09$ \\
\hline $3 p 3 d-3 s 5 d$ & 3Po-3De & $5: 5-5: 18$ & 60.37 & $2.05 \mathrm{E}-04$ & $2.04 \mathrm{E}-04$ & $3.75 \mathrm{E}+08$ \\
\hline $3 p 3 d-3 s 5 d$ & 3Po-3De & $5: 5-7: 10$ & 60.36 & $1.36 \mathrm{E}-03$ & $1.35 \mathrm{E}-03$ & $1.78 \mathrm{E}+09$ \\
\hline$L S$ & 3Po-3De & $9-15$ & & $2.20 \mathrm{E}-03$ & $3.97 \mathrm{E}-03$ & $2.39 \mathrm{E}+09$ \\
\hline $3 s 3 p-3 s 3 d$ & 1Po-3De & $3: 2-3: 2$ & 305.94 & $3.69 \mathrm{E}-04$ & $1.11 \mathrm{E}-03$ & $2.63 \mathrm{E}+07$ \\
\hline $3 s 3 p-3 s 3 d$ & 1Po-3De & $3: 2-5: 3$ & 304.99 & $5.11 \mathrm{E}-04$ & $1.54 \mathrm{E}-03$ & $2.20 \mathrm{E}+07$ \\
\hline $3 s 3 d-3 p 3 d$ & 3De-3Do & $3: 2-3: 3$ & 328.83 & $3.52 \mathrm{E}-02$ & $1.14 \mathrm{E}-01$ & $2.17 \mathrm{E}+09$ \\
\hline $3 s 3 d-3 p 3 d$ & 3De-3Do & $3: 2-5: 4$ & 314.61 & $1.54 \mathrm{E}-02$ & $4.77 \mathrm{E}-02$ & $6.21 \mathrm{E}+08$ \\
\hline $3 s 3 d-3 p 3 d$ & 3De-3Do & $5: 3-3: 3$ & 329.94 & $6.10 \mathrm{E}-02$ & $3.32 \mathrm{E}-01$ & $6.23 \mathrm{E}+09$ \\
\hline $3 s 3 d-3 p 3 d$ & 3De-3Do & $5: 3-5: 4$ & 315.62 & $7.70 \mathrm{E}-03$ & $4.00 \mathrm{E}-02$ & $5.16 \mathrm{E}+08$ \\
\hline $3 s 3 d-3 p 3 d$ & 3De-3Do & $5: 3-7: 2$ & 317.39 & $3.60 \mathrm{E}-02$ & $1.88 \mathrm{E}-01$ & $1.70 \mathrm{E}+09$ \\
\hline $3 s 3 d-3 p 3 d$ & 3De-3Do & $7: 1-5: 4$ & 317.25 & $9.09 \mathrm{E}-02$ & $6.65 \mathrm{E}-01$ & $8.44 \mathrm{E}+09$ \\
\hline $3 s 3 d-3 p 3 d$ & 3De-3Do & $7: 1-7: 2$ & 319.04 & $1.14 \mathrm{E}-01$ & $8.36 \mathrm{E}-01$ & $7.45 \mathrm{E}+09$ \\
\hline$L S$ & 3De-3Do & $15-15$ & & $1.41 \mathrm{E}-01$ & $2.22 \mathrm{E}+00$ & $9.16 \mathrm{E}+09$ \\
\hline $3 s 3 d-3 p 3 d$ & 3De-1Po & $3: 2-3: 5$ & 252.45 & $4.40 \mathrm{E}-04$ & $1.10 \mathrm{E}-03$ & $4.61 \mathrm{E}+07$ \\
\hline $3 s 3 d-3 p 3 d$ & 3De-1Po & $5: 3-3: 5$ & 253.10 & $7.93 \mathrm{E}-04$ & $3.30 \mathrm{E}-03$ & $1.38 \mathrm{E}+08$ \\
\hline $3 s 3 d-3 s 4 p$ & 3De-1Po & $3: 2-3: 7$ & 82.56 & $5.08 \mathrm{E}-03$ & $4.14 \mathrm{E}-03$ & $4.97 \mathrm{E}+09$ \\
\hline $3 s 3 d-3 s 4 p$ & $3 \mathrm{De}-1$ Po & $5: 3-3: 7$ & 82.63 & $9.06 \mathrm{E}-03$ & $1.23 \mathrm{E}-02$ & $1.47 \mathrm{E}+10$ \\
\hline $3 s 3 d-3 s 5 p$ & 3De-1Po & $3: 2-3: 15$ & 52.96 & $1.63 \mathrm{E}-02$ & $8.53 \mathrm{E}-03$ & $3.88 \mathrm{E}+10$ \\
\hline $3 s 3 d-3 s 5 p$ & 3De-1Po & $5: 3-3: 15$ & 52.99 & $2.23 \mathrm{E}-05$ & $1.94 \mathrm{E}-05$ & $8.83 E+07$ \\
\hline $3 s 3 p-3 s 4 s$ & $1 \mathrm{Po}-3 \mathrm{Se}$ & $3: 2-3: 4$ & 70.83 & $6.32 \mathrm{E}-04$ & $4.42 \mathrm{E}-04$ & $8.40 \mathrm{E}+08$ \\
\hline $3 p 3 d-3 s 4 s$ & 3Do-3Se & $3: 3-3: 4$ & 128.07 & $7.78 \mathrm{E}-06$ & $9.84 \mathrm{E}-06$ & $3.16 \mathrm{E}+06$ \\
\hline
\end{tabular}


Table 2. (continued)

\begin{tabular}{|c|c|c|c|c|c|c|}
\hline$C_{i}-C_{k}$ & $T_{i}-T_{k}$ & $g_{i}: \mathrm{I}-g_{j}: \mathrm{K}$ & $E_{i k}(\AA)$ & $f$ & $S$ & $A\left(\mathrm{~s}^{-1}\right)$ \\
\hline $3 p 3 d-3 s 4 s$ & 3Do-3Se & $5: 4-3: 4$ & 130.37 & $2.37 \mathrm{E}-05$ & $5.08 \mathrm{E}-05$ & $1.55 E+07$ \\
\hline$L S$ & 3Do-3Se & $15-3$ & & $9.46 \mathrm{E}-06$ & $6.06 \mathrm{E}-05$ & $1.87 \mathrm{E}+07$ \\
\hline $3 p 3 d-3 s 4 s$ & 1Po-3Se & $3: 5-3: 4$ & 145.18 & $2.79 \mathrm{E}-06$ & $4.00 \mathrm{E}-06$ & $8.83 E+05$ \\
\hline $3 s 4 s-3 s 4 p$ & $3 \mathrm{Se}-1 \mathrm{Po}$ & $3: 4-3: 7$ & 791.92 & $5.28 \mathrm{E}-02$ & $4.13 \mathrm{E}-01$ & $5.62 \mathrm{E}+08$ \\
\hline $3 s 4 s-3 s 5 p$ & $3 \mathrm{Se}-1 \mathrm{Po}$ & $3: 4-3: 15$ & 124.49 & $1.93 \mathrm{E}-02$ & $2.37 \mathrm{E}-02$ & $8.29 E+09$ \\
\hline $3 s 3 p-3 s 4 d$ & 1Po-3De & $3: 2-3: 5$ & 59.54 & $5.16 \mathrm{E}-04$ & $3.04 \mathrm{E}-04$ & $9.71 E+08$ \\
\hline $3 s 3 p-3 s 4 d$ & 1Po-3De & $3: 2-5: 8$ & 59.52 & $1.30 \mathrm{E}-03$ & $7.64 \mathrm{E}-04$ & $1.47 \mathrm{E}+09$ \\
\hline $3 p 3 d-3 s 4 d$ & 3Do-3De & $3: 3-3: 5$ & 95.38 & $7.84 \mathrm{E}-07$ & $7.39 \mathrm{E}-07$ & $5.75 E+05$ \\
\hline $3 p 3 d-3 s 4 d$ & 3Do-3De & $5: 4-3: 5$ & 96.65 & $1.40 \mathrm{E}-05$ & $2.23 \mathrm{E}-05$ & $1.67 \mathrm{E}+07$ \\
\hline $3 p 3 d-3 s 4 d$ & 3Do-3De & $3: 3-5: 8$ & 95.32 & $4.16 \mathrm{E}-05$ & $3.92 \mathrm{E}-05$ & $1.83 \mathrm{E}+07$ \\
\hline $3 p 3 d-3 s 4 d$ & 3Do-3De & $5: 4-5: 8$ & 96.58 & $6.55 \mathrm{E}-06$ & $1.04 \mathrm{E}-05$ & $4.68 \mathrm{E}+06$ \\
\hline $3 p 3 d-3 s 4 d$ & 3Do-3De & $7: 2-5: 8$ & 96.42 & $6.14 \mathrm{E}-05$ & $1.37 \mathrm{E}-04$ & $6.17 E+07$ \\
\hline $3 p 3 d-3 s 4 d$ & 3Do-3De & $5: 4-7: 3$ & 96.47 & $6.65 \mathrm{E}-05$ & $1.06 \mathrm{E}-04$ & $3.41 \mathrm{E}+07$ \\
\hline $3 p 3 d-3 s 4 d$ & 3Do-3De & $7: 2-7: 3$ & 96.31 & $1.09 \mathrm{E}-05$ & $2.43 \mathrm{E}-05$ & $7.87 \mathrm{E}+06$ \\
\hline$L S$ & 3Do-3De & 15-15 & & $7.12 \mathrm{E}-05$ & $3.40 \mathrm{E}-04$ & $5.09 E+07$ \\
\hline $3 p 3 d-3 s 4 d$ & 1Po-3De & $3: 5-3: 5$ & 104.56 & $4.54 \mathrm{E}-06$ & $4.69 \mathrm{E}-06$ & $2.77 \mathrm{E}+06$ \\
\hline $3 p 3 d-3 s 4 d$ & 1Po-3De & $3: 5-5: 8$ & 104.48 & $5.08 \mathrm{E}-06$ & $5.24 \mathrm{E}-06$ & $1.86 \mathrm{E}+06$ \\
\hline $3 s 4 p-3 s 4 d$ & 1Po-3De & $3: 7-3: 5$ & 707.51 & $2.72 \mathrm{E}-02$ & $1.90 \mathrm{E}-01$ & $3.62 \mathrm{E}+08$ \\
\hline $3 s 4 p-3 s 4 d$ & 1Po-3De & $3: 7-5: 8$ & 704.01 & $3.59 \mathrm{E}-02$ & $2.50 \mathrm{E}-01$ & $2.90 \mathrm{E}+08$ \\
\hline $3 s 4 d-3 s 5 p$ & 3De-1Po & $3: 5-3: 15$ & 186.68 & $1.21 \mathrm{E}-02$ & $2.23 \mathrm{E}-02$ & $2.31 E+09$ \\
\hline $3 s 4 d-3 s 5 p$ & 3De-1Po & $5: 8-3: 15$ & 186.92 & $9.65 \mathrm{E}-03$ & $2.97 \mathrm{E}-02$ & $3.07 \mathrm{E}+09$ \\
\hline $3 s 3 p-3 p 4 f$ & 1Po-3De & $3: 2-3: 10$ & 48.35 & $3.06 \mathrm{E}-07$ & $1.46 \mathrm{E}-07$ & $8.74 E+05$ \\
\hline $3 s 3 p-3 p 4 f$ & 1Po-3De & $3: 2-5: 14$ & 48.49 & $9.28 \mathrm{E}-05$ & $4.45 \mathrm{E}-05$ & $1.58 \mathrm{E}+08$ \\
\hline $3 p 3 d-3 p 4 f$ & 3Do-3De & $3: 3-3: 10$ & 69.58 & $6.94 \mathrm{E}-04$ & $4.77 \mathrm{E}-04$ & $9.56 \mathrm{E}+08$ \\
\hline $3 p 3 d-3 p 4 f$ & 3Do-3De & $5: 4-3: 10$ & 70.25 & $5.85 \mathrm{E}-04$ & $6.76 \mathrm{E}-04$ & $1.32 \mathrm{E}+09$ \\
\hline $3 p 3 d-3 p 4 f$ & 3Do-3De & $3: 3-5: 14$ & 69.86 & $8.19 \mathrm{E}-02$ & $5.65 \mathrm{E}-02$ & $6.72 \mathrm{E}+10$ \\
\hline $3 p 3 d-3 p 4 f$ & 3Do-3De & $5: 4-5: 14$ & 70.54 & $2.43 \mathrm{E}-05$ & $2.83 \mathrm{E}-05$ & $3.26 E+07$ \\
\hline $3 p 3 d-3 p 4 f$ & 3Do-3De & $7: 2-5: 14$ & 70.45 & $1.65 \mathrm{E}-02$ & $2.68 \mathrm{E}-02$ & $3.11 E+10$ \\
\hline $3 p 3 d-3 p 4 f$ & 3Do-3De & $5: 4-7: 8$ & 70.60 & $2.40 \mathrm{E}-01$ & $2.79 \mathrm{E}-01$ & $2.30 \mathrm{E}+11$ \\
\hline $3 p 3 d-3 p 4 f$ & 3Do-3De & $7: 2-7: 8$ & 70.51 & $1.40 \mathrm{E}-01$ & $2.28 \mathrm{E}-01$ & $1.88 \mathrm{E}+11$ \\
\hline$L S$ & 3Do-3De & $15-15$ & & $1.70 \mathrm{E}-01$ & $5.91 \mathrm{E}-01$ & $2.27 \mathrm{E}+11$ \\
\hline $3 p 3 d-3 p 4 f$ & 1Po-3De & $3: 5-3: 10$ & 74.34 & $1.25 \mathrm{E}-06$ & $9.15 \mathrm{E}-07$ & $1.50 \mathrm{E}+06$ \\
\hline $3 p 3 d-3 p 4 f$ & 1Po-3De & $3: 5-5: 14$ & 74.66 & $1.43 \mathrm{E}-02$ & $1.05 \mathrm{E}-02$ & $1.03 \mathrm{E}+10$ \\
\hline $3 s 4 p-3 p 4 f$ & 1Po-3De & $3: 7-3: 10$ & 188.63 & $9.77 \mathrm{E}-05$ & $1.82 \mathrm{E}-04$ & $1.83 \mathrm{E}+07$ \\
\hline $3 s 4 p-3 p 4 f$ & 1Po-3De & $3: 7-5: 14$ & 190.72 & $1.34 \mathrm{E}-04$ & $2.53 \mathrm{E}-04$ & $1.48 \mathrm{E}+07$ \\
\hline $3 p 4 f-3 s 5 p$ & 3De-1Po & $3: 10-3: 15$ & 680.76 & $6.48 \mathrm{E}-04$ & $4.36 \mathrm{E}-03$ & $9.33 E+06$ \\
\hline $3 p 4 f-3 s 5 p$ & 3De-1Po & $5: 14-3: 15$ & 654.88 & $7.10 \mathrm{E}-05$ & $7.65 \mathrm{E}-04$ & $1.84 \mathrm{E}+06$ \\
\hline $3 s 3 p-3 s 5 s$ & 1Po-3Se & $3: 2-3: 12$ & 45.60 & $1.06 \mathrm{E}-04$ & $4.75 \mathrm{E}-05$ & $3.39 E+08$ \\
\hline $3 p 3 d-3 s 5 s$ & 3Do-3Se & $3: 3-3: 12$ & 64.02 & $4.16 \mathrm{E}-05$ & $2.63 \mathrm{E}-05$ & $6.77 \mathrm{E}+07$ \\
\hline $3 p 3 d-3 s 5 s$ & 3Do-3Se & $5: 4-3: 12$ & 64.59 & $9.83 \mathrm{E}-05$ & $1.04 \mathrm{E}-04$ & $2.62 E+08$ \\
\hline$L S$ & 3Do-3Se & $15-3$ & & $4.11 \mathrm{E}-05$ & $1.30 \mathrm{E}-04$ & $3.32 \mathrm{E}+08$ \\
\hline $3 p 3 d-3 s 5 s$ & 1Po-3Se & $3: 5-3: 12$ & 68.03 & $1.24 \mathrm{E}-06$ & $8.34 \mathrm{E}-07$ & $1.79 \mathrm{E}+06$ \\
\hline $3 s 4 p-3 s 5 s$ & 1Po-3Se & $3: 7-3: 12$ & 152.71 & $3.42 \mathrm{E}-02$ & $5.16 \mathrm{E}-02$ & $9.78 \mathrm{E}+09$ \\
\hline $3 s 5 s-3 s 5 p$ & 3Se-1Po & $3: 12-3: 15$ & 4504.55 & $2.91 \mathrm{E}-02$ & $1.29 \mathrm{E}+00$ & $9.56 \mathrm{E}+06$ \\
\hline $3 s 3 p-3 s 5 d$ & 1Po-3De & $3: 2-3: 13$ & 43.70 & $1.86 \mathrm{E}-04$ & $8.03 \mathrm{E}-05$ & $6.50 \mathrm{E}+08$ \\
\hline $3 s 3 p-3 s 5 d$ & 1Po-3De & $3: 2-5: 18$ & 43.71 & $1.75 \mathrm{E}-04$ & $7.54 \mathrm{E}-05$ & $3.66 \mathrm{E}+08$ \\
\hline $3 p 3 d-3 s 5 d$ & 3Do-3De & $3: 3-3: 13$ & 60.34 & $2.23 \mathrm{E}-04$ & $1.33 \mathrm{E}-04$ & $4.08 \mathrm{E}+08$ \\
\hline $3 p 3 d-3 s 5 d$ & 3Do-3De & $5: 4-3: 13$ & 60.85 & $1.72 \mathrm{E}-05$ & $1.72 \mathrm{E}-05$ & $5.16 \mathrm{E}+07$ \\
\hline $3 p 3 d-3 s 5 d$ & 3Do-3De & $3: 3-5: 18$ & 60.35 & $5.25 \mathrm{E}-04$ & $3.13 \mathrm{E}-04$ & $5.77 \mathrm{E}+08$ \\
\hline $3 p 3 d-3 s 5 d$ & 3Do-3De & $5: 4-5: 18$ & 60.85 & $2.55 \mathrm{E}-04$ & $2.55 \mathrm{E}-04$ & $4.58 \mathrm{E}+08$ \\
\hline $3 p 3 d-3 s 5 d$ & 3Do-3De & $7: 2-5: 18$ & 60.79 & $1.61 \mathrm{E}-08$ & $2.25 \mathrm{E}-08$ & $4.06 \mathrm{E}+04$ \\
\hline $3 p 3 d-3 s 5 d$ & 3Do-3De & $5: 4-7: 10$ & 60.84 & $1.31 \mathrm{E}-03$ & $1.31 \mathrm{E}-03$ & $1.69 \mathrm{E}+09$ \\
\hline $3 p 3 d-3 s 5 d$ & 3Do-3De & $7: 2-7: 10$ & 60.77 & $3.80 \mathrm{E}-06$ & $5.32 \mathrm{E}-06$ & $6.86 \mathrm{E}+06$ \\
\hline$L S$ & 3Do-3De & $15-15$ & & $6.79 \mathrm{E}-04$ & $2.03 \mathrm{E}-03$ & $1.23 E+09$ \\
\hline $3 p 3 d-3 s 5 d$ & 1Po-3De & $3: 5-3: 13$ & 63.89 & $4.62 \mathrm{E}-06$ & $2.92 \mathrm{E}-06$ & $7.56 \mathrm{E}+06$ \\
\hline $3 p 3 d-3 s 5 d$ & 1Po-3De & $3: 5-5: 18$ & 63.90 & $2.07 \mathrm{E}-05$ & $1.30 \mathrm{E}-05$ & $2.02 \mathrm{E}+07$ \\
\hline $3 s 4 p-3 s 5 d$ & 1Po-3De & $3: 7-3: 13$ & 133.31 & $1.63 \mathrm{E}-02$ & $2.14 \mathrm{E}-02$ & $6.11 \mathrm{E}+09$ \\
\hline $3 s 4 p-3 s 5 d$ & 1Po-3De & $3: 7-5: 18$ & 133.35 & $2.80 \mathrm{E}-02$ & $3.68 \mathrm{E}-02$ & $6.29 E+09$ \\
\hline $3 s 5 p-3 s 5 d$ & 1Po-3De & $3: 15-3: 13$ & 1367.94 & $4.78 \mathrm{E}-02$ & $6.45 \mathrm{E}-01$ & $1.70 \mathrm{E}+08$ \\
\hline $3 s 5 p-3 s 5 d$ & 1Po-3De & $3: 15-5: 18$ & 1371.69 & $9.48 \mathrm{E}-02$ & $1.28 \mathrm{E}+00$ & $2.02 \mathrm{E}+08$ \\
\hline $3 p^{2}-3 p 3 d$ & 3Pe-3Fo & $3: 1-5: 2$ & 274.99 & $5.00 \mathrm{E}-04$ & $1.36 \mathrm{E}-03$ & $2.65 \mathrm{E}+07$ \\
\hline $3 p^{2}-3 p 3 d$ & $3 \mathrm{Pe}-3 \mathrm{Fo}$ & $5: 2-5: 2$ & 288.65 & $1.63 \mathrm{E}-03$ & $7.75 \mathrm{E}-03$ & $1.30 \mathrm{E}+08$ \\
\hline $3 p^{2}-3 p 3 d$ & $3 \mathrm{Pe}-3 \mathrm{Fo}$ & $5: 2-7: 1$ & 280.65 & $1.80 \mathrm{E}-03$ & $8.32 \mathrm{E}-03$ & $1.09 \mathrm{E}+08$ \\
\hline$L S$ & $3 \mathrm{Pe}-3 \mathrm{Fo}$ & $9-21$ & & $2.07 \mathrm{E}-03$ & $1.74 \mathrm{E}-02$ & $7.35 E+07$ \\
\hline $3 p^{2}-3 p 3 d$ & 3Pe-1Do & $3: 1-5: 3$ & 260.47 & $9.05 \mathrm{E}-03$ & $2.33 \mathrm{E}-02$ & $5.34 \mathrm{E}+08$ \\
\hline $3 p^{2}-3 p 3 d$ & 3Pe-1Do & $5: 2-5: 3$ & 272.70 & $1.85 \mathrm{E}-02$ & $8.29 \mathrm{E}-02$ & $1.66 \mathrm{E}+09$ \\
\hline $3 p^{2}-3 s 4 f$ & $3 \mathrm{Pe}-3 \mathrm{Fo}$ & $3: 1-5: 8$ & 64.77 & $2.66 \mathrm{E}-06$ & $1.70 \mathrm{E}-06$ & $2.54 \mathrm{E}+06$ \\
\hline $3 p^{2}-3 s 4 f$ & $3 \mathrm{Pe}-3 \mathrm{Fo}$ & $5: 2-5: 8$ & 65.50 & $1.32 \mathrm{E}-06$ & $1.42 \mathrm{E}-06$ & $2.05 E+06$ \\
\hline $3 p^{2}-3 s 4 f$ & $3 \mathrm{Pe}-3 \mathrm{Fo}$ & $5: 2-7: 4$ & 65.50 & $3.12 \mathrm{E}-05$ & $3.36 \mathrm{E}-05$ & $3.46 \mathrm{E}+07$ \\
\hline$L S$ & $3 \mathrm{Pe}-3 \mathrm{Fo}$ & $9-21$ & & $1.90 \mathrm{E}-05$ & $3.67 \mathrm{E}-05$ & $1.27 \mathrm{E}+07$ \\
\hline $3 p^{2}-3 s 5 f$ & $3 \mathrm{Pe}-3 \mathrm{Fo}$ & $3: 1-5: 18$ & 47.35 & $2.22 \mathrm{E}-07$ & $1.04 \mathrm{E}-07$ & $3.96 \mathrm{E}+05$ \\
\hline $3 p^{2}-3 s 5 f$ & $3 \mathrm{Pe}-3 \mathrm{Fo}$ & $5: 2-5: 18$ & 47.74 & $9.56 \mathrm{E}-08$ & $7.52 \mathrm{E}-08$ & $2.80 \mathrm{E}+05$ \\
\hline $3 p^{2}-3 s 5 f$ & $3 \mathrm{Pe}-3 \mathrm{Fo}$ & $5: 2-7: 12$ & 47.74 & $6.33 \mathrm{E}-06$ & $4.97 \mathrm{E}-06$ & $1.32 \mathrm{E}+07$ \\
\hline$L S$ & $3 \mathrm{Pe}-3 \mathrm{Fo}$ & $9-21$ & & $3.64 \mathrm{E}-06$ & $5.15 \mathrm{E}-06$ & $4.58 \mathrm{E}+06$ \\
\hline $3 s 3 d-3 p 3 d$ & $3 \mathrm{De}-3 \mathrm{Fo}$ & $3: 2-5: 2$ & 400.84 & $1.23 \mathrm{E}-01$ & $4.86 \mathrm{E}-01$ & $3.06 E+09$ \\
\hline $3 s 3 d-3 p 3 d$ & 3De-3Fo & $5: 3-5: 2$ & 402.49 & $1.98 \mathrm{E}-02$ & $1.31 \mathrm{E}-01$ & $8.15 E+08$ \\
\hline
\end{tabular}


Table 2. (continued)

\begin{tabular}{|c|c|c|c|c|c|c|}
\hline$C_{i}-C_{k}$ & $T_{i}-T_{k}$ & $g_{i}: I-g_{j}: K$ & $E_{i k}(\AA)$ & $f$ & $S$ & $A\left(\mathrm{~s}^{-1}\right)$ \\
\hline $3 s 3 d-3 p 3 d$ & 3De-3Fo & $5: 3-7: 1$ & 387.10 & $1.27 \mathrm{E}-01$ & $8.06 \mathrm{E}-01$ & $4.02 \mathrm{E}+09$ \\
\hline $3 s 3 d-3 p 3 d$ & 3De-3Fo & $7: 1-5: 2$ & 405.13 & $2.00 \mathrm{E}-04$ & $1.87 \mathrm{E}-03$ & $1.14 \mathrm{E}+07$ \\
\hline $3 s 3 d-3 p 3 d$ & 3De-3Fo & $7: 1-7: 1$ & 389.55 & $2.00 \mathrm{E}-02$ & $1.80 \mathrm{E}-01$ & $8.81 \mathrm{E}+08$ \\
\hline $3 s 3 d-3 p 3 d$ & 3De-3Fo & $7: 1-9: 1$ & 372.80 & $1.49 \mathrm{E}-01$ & $1.28 \mathrm{E}+00$ & $5.55 \mathrm{E}+09$ \\
\hline$L S$ & 3De-3Fo & $15-21$ & & $1.52 \mathrm{E}-01$ & $2.88 \mathrm{E}+00$ & $4.95 \mathrm{E}+09$ \\
\hline $3 s 3 d-3 p 3 d$ & 3De-1Do & $3: 2-5: 3$ & 370.72 & $1.75 \mathrm{E}-02$ & $6.39 \mathrm{E}-02$ & $5.08 \mathrm{E}+08$ \\
\hline $3 s 3 d-3 p 3 d$ & 3De-1Do & $5: 3-5: 3$ & 372.13 & $2.33 \mathrm{E}-03$ & $1.43 \mathrm{E}-02$ & $1.12 \mathrm{E}+08$ \\
\hline $3 s 3 d-3 p 3 d$ & 3De-1Do & $7: 1-5: 3$ & 374.39 & $4.23 \mathrm{E}-03$ & $3.65 \mathrm{E}-02$ & $2.82 \mathrm{E}+08$ \\
\hline $3 s 3 d-3 s 4 f$ & 3De-3Fo & $3: 2-5: 8$ & 69.94 & $8.93 \mathrm{E}-01$ & $6.17 \mathrm{E}-01$ & $7.31 \mathrm{E}+11$ \\
\hline $3 s 3 d-3 s 4 f$ & 3De-3Fo & $5: 3-5: 8$ & 69.99 & $9.93 \mathrm{E}-02$ & $1.14 \mathrm{E}-01$ & $1.35 \mathrm{E}+11$ \\
\hline $3 s 3 d-3 s 4 f$ & 3De-3Fo & $5: 3-7: 4$ & 69.99 & $7.95 \mathrm{E}-01$ & $9.16 \mathrm{E}-01$ & $7.73 E+11$ \\
\hline $3 s 3 d-3 s 4 f$ & 3De-3Fo & $7: 1-5: 8$ & 70.07 & $2.03 \mathrm{E}-03$ & $3.28 \mathrm{E}-03$ & $3.86 \mathrm{E}+09$ \\
\hline $3 s 3 d-3 s 4 f$ & 3De-3Fo & $7: 1-7: 4$ & 70.07 & $7.11 \mathrm{E}-02$ & $1.15 \mathrm{E}-01$ & $9.65 E+10$ \\
\hline $3 s 3 d-3 s 4 f$ & 3De-3Fo & $7: 1-9: 2$ & 70.05 & $8.23 \mathrm{E}-01$ & $1.33 \mathrm{E}+00$ & $8.70 \mathrm{E}+11$ \\
\hline$L S$ & 3De-3Fo & $15-21$ & & $8.95 \mathrm{E}-01$ & $3.10 \mathrm{E}+00$ & $8.69 E+11$ \\
\hline $3 s 3 d-3 s 5 f$ & 3De-3Fo & $3: 2-5: 18$ & 50.06 & $1.71 \mathrm{E}-01$ & $8.44 \mathrm{E}-02$ & $2.73 E+11$ \\
\hline $3 s 3 d-3 s 5 f$ & 3De-3Fo & $5: 3-5: 18$ & 50.08 & $1.90 \mathrm{E}-02$ & $1.56 \mathrm{E}-02$ & $5.04 \mathrm{E}+10$ \\
\hline $3 s 3 d-3 s 5 f$ & $3 \mathrm{De}-3 \mathrm{Fo}$ & $5: 3-7: 12$ & 50.08 & $1.52 \mathrm{E}-01$ & $1.25 \mathrm{E}-01$ & $2.88 \mathrm{E}+11$ \\
\hline $3 s 3 d-3 s 5 f$ & 3De-3Fo & $7: 1-5: 18$ & 50.13 & $3.87 \mathrm{E}-04$ & $4.48 \mathrm{E}-04$ & $1.44 \mathrm{E}+09$ \\
\hline $3 s 3 d-3 s 5 f$ & 3De-3Fo & $7: 1-7: 12$ & 50.13 & $1.35 \mathrm{E}-02$ & $1.56 \mathrm{E}-02$ & $3.60 \mathrm{E}+10$ \\
\hline $3 s 3 d-3 s 5 f$ & 3De-3Fo & $7: 1-9: 5$ & 50.12 & $1.57 \mathrm{E}-01$ & $1.81 \mathrm{E}-01$ & $3.24 \mathrm{E}+11$ \\
\hline$L S$ & 3De-3Fo & $15-21$ & & $1.71 \mathrm{E}-01$ & $4.22 \mathrm{E}-01$ & $3.26 \mathrm{E}+11$ \\
\hline $3 p 3 d-3 s 4 s$ & 3Fo-3Se & $5: 2-3: 4$ & 119.70 & $3.62 \mathrm{E}-09$ & $7.13 \mathrm{E}-09$ & $2.81 \mathrm{E}+03$ \\
\hline $3 p 3 d-3 s 4 s$ & 1Do-3Se & $5: 3-3: 4$ & 122.67 & $5.75 \mathrm{E}-07$ & $1.16 \mathrm{E}-06$ & $4.25 \mathrm{E}+05$ \\
\hline $3 s 4 s-3 s 4 f$ & $3 \mathrm{Se}-3 \mathrm{Fo}$ & $3: 4-5: 8$ & 290.01 & $1.30 \mathrm{E}-11$ & $3.72 \mathrm{E}-11$ & $6.18 \mathrm{E}-01$ \\
\hline $3 s 4 s-3 s 5 f$ & $3 \mathrm{Se}-3 \mathrm{Fo}$ & $3: 4-5: 18$ & 109.56 & $2.54 \mathrm{E}-11$ & $2.75 \mathrm{E}-11$ & $8.47 \mathrm{E}+00$ \\
\hline $3 p 3 d-3 s 4 d$ & 3Fo-3De & $5: 2-3: 5$ & 90.66 & $2.99 \mathrm{E}-03$ & $4.47 \mathrm{E}-03$ & $4.05 E+09$ \\
\hline $3 p 3 d-3 s 4 d$ & 3Fo-3De & $5: 2-5: 8$ & 90.60 & $5.00 \mathrm{E}-04$ & $7.45 \mathrm{E}-04$ & $4.06 \mathrm{E}+08$ \\
\hline $3 p 3 d-3 s 4 d$ & 3Fo-3De & $7: 1-5: 8$ & 91.42 & $2.95 \mathrm{E}-03$ & $6.21 \mathrm{E}-03$ & $3.30 \mathrm{E}+09$ \\
\hline $3 p 3 d-3 s 4 d$ & 3Fo-3De & $5: 2-7: 3$ & 90.50 & $9.79 \mathrm{E}-06$ & $1.46 \mathrm{E}-05$ & $5.69 \mathrm{E}+06$ \\
\hline $3 p 3 d-3 s 4 d$ & 3Fo-3De & $7: 1-7: 3$ & 91.32 & $3.68 \mathrm{E}-04$ & $7.74 \mathrm{E}-04$ & $2.94 \mathrm{E}+08$ \\
\hline $3 p 3 d-3 s 4 d$ & $3 \mathrm{Fo}-3 \mathrm{De}$ & $9: 1-7: 3$ & 92.29 & $3.13 \mathrm{E}-03$ & $8.57 \mathrm{E}-03$ & $3.15 E+09$ \\
\hline$L S$ & 3Fo-3De & $21-15$ & & $3.28 \mathrm{E}-03$ & $2.08 \mathrm{E}-02$ & $3.65 E+09$ \\
\hline $3 p 3 d-3 s 4 d$ & 1Do-3De & $5: 3-3: 5$ & 92.35 & $3.20 \mathrm{E}-04$ & $4.87 \mathrm{E}-04$ & $4.18 \mathrm{E}+08$ \\
\hline $3 p 3 d-3 s 4 d$ & 1Do-3De & $5: 3-5: 8$ & 92.29 & $4.92 \mathrm{E}-05$ & $7.47 \mathrm{E}-05$ & $3.85 E+07$ \\
\hline $3 p 3 d-3 s 4 d$ & 1Do-3De & $5: 3-7: 3$ & 92.19 & $1.42 \mathrm{E}-05$ & $2.15 \mathrm{E}-05$ & $7.96 \mathrm{E}+06$ \\
\hline $3 s 4 d-3 s 4 f$ & 3De-3Fo & $3: 5-5: 8$ & 1295.33 & $1.38 \mathrm{E}-01$ & $1.76 \mathrm{E}+00$ & $3.29 \mathrm{E}+08$ \\
\hline $3 s 4 d-3 s 4 f$ & 3De-3Fo & $5: 8-5: 8$ & 1307.23 & $1.47 \mathrm{E}-02$ & $3.16 \mathrm{E}-01$ & $5.74 \mathrm{E}+07$ \\
\hline $3 s 4 d-3 s 4 f$ & 3De-3Fo & $5: 8-7: 4$ & 1305.35 & $1.19 \mathrm{E}-01$ & $2.56 \mathrm{E}+00$ & $3.33 E+08$ \\
\hline $3 s 4 d-3 s 4 f$ & $3 \mathrm{De}-3 \mathrm{Fo}$ & $7: 3-5: 8$ & 1327.41 & $3.03 \mathrm{E}-04$ & $9.27 \mathrm{E}-03$ & $1.61 \mathrm{E}+06$ \\
\hline $3 s 4 d-3 s 4 f$ & 3De-3Fo & $7: 3-7: 4$ & 1325.48 & $1.07 \mathrm{E}-02$ & $3.26 \mathrm{E}-01$ & $4.06 \mathrm{E}+07$ \\
\hline $3 s 4 d-3 s 4 f$ & $3 \mathrm{De}-3 \mathrm{Fo}$ & $7: 3-9: 2$ & 1321.06 & $1.25 \mathrm{E}-01$ & $3.81 \mathrm{E}+00$ & $3.72 \mathrm{E}+08$ \\
\hline$L S$ & $3 \mathrm{De}-3 \mathrm{Fo}$ & $15-21$ & & $1.36 \mathrm{E}-01$ & $8.78 \mathrm{E}+00$ & $3.76 \mathrm{E}+08$ \\
\hline $3 s 4 d-3 s 5 f$ & $3 \mathrm{De}-3 \mathrm{Fo}$ & $3: 5-5: 18$ & 155.02 & $6.53 \mathrm{E}-01$ & $9.99 \mathrm{E}-01$ & $1.09 \mathrm{E}+11$ \\
\hline $3 s 4 d-3 s 5 f$ & 3De-3Fo & $5: 8-5: 18$ & 155.19 & $7.08 \mathrm{E}-02$ & $1.81 \mathrm{E}-01$ & $1.96 \mathrm{E}+10$ \\
\hline $3 s 4 d-3 s 5 f$ & 3De-3Fo & $5: 8-7: 12$ & 155.19 & $5.70 \mathrm{E}-01$ & $1.46 \mathrm{E}+00$ & $1.13 \mathrm{E}+11$ \\
\hline $3 s 4 d-3 s 5 f$ & $3 \mathrm{De}-3 \mathrm{Fo}$ & $7: 3-5: 18$ & 155.47 & $1.50 \mathrm{E}-03$ & $5.36 \mathrm{E}-03$ & $5.78 \mathrm{E}+08$ \\
\hline $3 s 4 d-3 s 5 f$ & $3 \mathrm{De}-3 \mathrm{Fo}$ & $7: 3-7: 12$ & 155.47 & $5.24 \mathrm{E}-02$ & $1.88 \mathrm{E}-01$ & $1.45 \mathrm{E}+10$ \\
\hline $3 s 4 d-3 s 5 f$ & 3De-3Fo & $7: 3-9: 5$ & 155.42 & $6.07 \mathrm{E}-01$ & $2.18 \mathrm{E}+00$ & $1.30 \mathrm{E}+11$ \\
\hline$L S$ & 3De-3Fo & $15-21$ & & $6.53 \mathrm{E}-01$ & $5.01 \mathrm{E}+00$ & $1.28 \mathrm{E}+11$ \\
\hline $3 p 3 d-3 p 4 f$ & 3Fo-3De & $5: 2-3: 10$ & 67.03 & $3.98 \mathrm{E}-03$ & $4.39 \mathrm{E}-03$ & $9.84 \mathrm{E}+09$ \\
\hline $3 p 3 d-3 p 4 f$ & 3Fo-3De & $5: 2-5: 14$ & 67.29 & $1.69 \mathrm{E}-05$ & $1.87 \mathrm{E}-05$ & $2.48 \mathrm{E}+07$ \\
\hline $3 p 3 d-3 p 4 f$ & 3Fo-3De & $7: 1-5: 14$ & 67.74 & $6.96 \mathrm{E}-03$ & $1.09 \mathrm{E}-02$ & $1.42 \mathrm{E}+10$ \\
\hline $3 p 3 d-3 p 4 f$ & 3Fo-3De & $5: 2-7: 8$ & 67.35 & $4.96 \mathrm{E}-04$ & $5.50 \mathrm{E}-04$ & $5.21 \mathrm{E}+08$ \\
\hline $3 p 3 d-3 p 4 f$ & 3Fo-3De & $7: 1-7: 8$ & 67.80 & $1.39 \mathrm{E}-03$ & $2.18 \mathrm{E}-03$ & $2.02 \mathrm{E}+09$ \\
\hline $3 p 3 d-3 p 4 f$ & $3 \mathrm{Fo}-3 \mathrm{De}$ & $9: 1-7: 8$ & 68.34 & $9.12 \mathrm{E}-03$ & $1.85 \mathrm{E}-02$ & $1.67 \mathrm{E}+10$ \\
\hline$L S$ & 3Fo-3De & $21-15$ & & $7.76 \mathrm{E}-03$ & $3.65 \mathrm{E}-02$ & $1.56 \mathrm{E}+10$ \\
\hline $3 p 3 d-3 p 4 f$ & 1Do-3De & $5: 3-3: 10$ & 67.95 & $9.44 \mathrm{E}-04$ & $1.06 \mathrm{E}-03$ & $2.27 \mathrm{E}+09$ \\
\hline $3 p 3 d-3 p 4 f$ & 1Do-3De & $5: 3-5: 14$ & 68.22 & $8.25 \mathrm{E}-03$ & $9.26 \mathrm{E}-03$ & $1.18 \mathrm{E}+10$ \\
\hline $3 p 3 d-3 p 4 f$ & 1Do-3De & $5: 3-7: 8$ & 68.28 & $2.72 \mathrm{E}-03$ & $3.06 \mathrm{E}-03$ & $2.78 \mathrm{E}+09$ \\
\hline $3 s 4 f-3 p 4 f$ & 3Fo-3De & $5: 8-3: 10$ & 320.94 & $9.22 \mathrm{E}-02$ & $4.87 \mathrm{E}-01$ & $9.95 \mathrm{E}+09$ \\
\hline $3 s 4 f-3 p 4 f$ & 3Fo-3De & $5: 8-5: 14$ & 327.03 & $5.36 \mathrm{E}-02$ & $2.89 \mathrm{E}-01$ & $3.34 \mathrm{E}+09$ \\
\hline $3 s 4 f-3 p 4 f$ & $3 \mathrm{Fo}-3 \mathrm{De}$ & $7: 4-5: 14$ & 327.15 & $6.62 \mathrm{E}-02$ & $4.99 \mathrm{E}-01$ & $5.78 \mathrm{E}+09$ \\
\hline $3 s 4 f-3 p 4 f$ & $3 F o-3 \mathrm{De}$ & $5: 8-7: 8$ & 328.42 & $5.22 \mathrm{E}-03$ & $2.82 \mathrm{E}-02$ & $2.31 \mathrm{E}+08$ \\
\hline $3 s 4 f-3 p 4 f$ & 3Fo-3De & $7: 4-7: 8$ & 328.54 & $4.90 \mathrm{E}-02$ & $3.71 \mathrm{E}-01$ & $3.03 E+09$ \\
\hline $3 s 4 f-3 p 4 f$ & 3Fo-3De & $9: 2-7: 8$ & 328.81 & $6.96 \mathrm{E}-02$ & $6.79 \mathrm{E}-01$ & $5.52 \mathrm{E}+09$ \\
\hline$L S$ & $3 \mathrm{Fo}-3 \mathrm{De}$ & $21-15$ & & $1.04 \mathrm{E}-01$ & $2.35 \mathrm{E}+00$ & $9.11 \mathrm{E}+09$ \\
\hline $3 p 4 f-3 s 5 f$ & 3De-3Fo & $3: 10-5: 18$ & 390.17 & $6.40 \mathrm{E}-03$ & $2.47 \mathrm{E}-02$ & $1.68 \mathrm{E}+08$ \\
\hline $3 p 4 f-3 s 5 f$ & 3De-3Fo & $5: 14-5: 18$ & 381.53 & $4.88 \mathrm{E}-04$ & $3.06 \mathrm{E}-03$ & $2.23 \mathrm{E}+07$ \\
\hline $3 p 4 f-3 s 5 f$ & 3De-3Fo & $5: 14-7: 12$ & 381.53 & $5.14 \mathrm{E}-03$ & $3.23 \mathrm{E}-02$ & $1.68 \mathrm{E}+08$ \\
\hline $3 p 4 f-3 s 5 f$ & 3De-3Fo & $7: 8-5: 18$ & 379.66 & $2.43 \mathrm{E}-06$ & $2.12 \mathrm{E}-05$ & $1.57 \mathrm{E}+05$ \\
\hline $3 p 4 f-3 s 5 f$ & $3 \mathrm{De}-3 \mathrm{Fo}$ & $7: 8-7: 12$ & 379.66 & $2.98 \mathrm{E}-04$ & $2.61 \mathrm{E}-03$ & $1.38 \mathrm{E}+07$ \\
\hline $3 p 4 f-3 s 5 f$ & 3De-3Fo & $7: 8-9: 5$ & 379.37 & $4.75 \mathrm{E}-03$ & $4.15 \mathrm{E}-02$ & $1.71 \mathrm{E}+08$ \\
\hline$L S$ & $3 \mathrm{De}-3 \mathrm{Fo}$ & $15-21$ & & $5.51 \mathrm{E}-03$ & $1.04 \mathrm{E}-01$ & $1.79 E+08$ \\
\hline $3 p 3 d-3 s 5 s$ & $3 \mathrm{Fo}-3 \mathrm{Se}$ & $5: 2-3: 12$ & 61.86 & $1.68 \mathrm{E}-07$ & $1.71 \mathrm{E}-07$ & $4.88 \mathrm{E}+05$ \\
\hline $3 p 3 d-3 s 5 s$ & 1Do-3Se & $5: 3-3: 12$ & 62.65 & $4.76 \mathrm{E}-06$ & $4.90 \mathrm{E}-06$ & $1.35 E+07$ \\
\hline
\end{tabular}


Table 2. (continued)

\begin{tabular}{|c|c|c|c|c|c|c|}
\hline$C_{i}-C_{k}$ & $T_{i}-T_{k}$ & $g_{i}: \mathrm{I}-g_{j}: \mathrm{K}$ & $E_{i k}(\AA)$ & $f$ & $S$ & $A\left(\mathrm{~s}^{-1}\right)$ \\
\hline $3 s 4 f-3 s 5 s$ & 3Fo-3Se & $5: 8-3: 12$ & 229.21 & $1.27 \mathrm{E}-10$ & $4.81 \mathrm{E}-10$ & $2.70 \mathrm{E}+01$ \\
\hline $3 s 5 s-3 s 5 f$ & $3 \mathrm{Se}-3 \mathrm{Fo}$ & $3: 12-5: 18$ & 759.86 & $5.09 \mathrm{E}-13$ & $3.82 \mathrm{E}-12$ & $3.53 \mathrm{E}-03$ \\
\hline $3 p 3 d-3 s 5 d$ & 3Fo-3De & $5: 2-3: 13$ & 58.42 & $1.59 \mathrm{E}-05$ & $1.53 \mathrm{E}-05$ & $5.19 \mathrm{E}+07$ \\
\hline $3 p 3 d-3 s 5 d$ & 3Fo-3De & $5: 2-5: 18$ & 58.42 & $1.92 \mathrm{E}-05$ & $1.85 \mathrm{E}-05$ & $3.76 \mathrm{E}+07$ \\
\hline $3 p 3 d-3 s 5 d$ & 3Fo-3De & $7: 1-5: 18$ & 58.76 & $2.52 \mathrm{E}-05$ & $3.41 \mathrm{E}-05$ & $6.81 \mathrm{E}+07$ \\
\hline $3 p 3 d-3 s 5 d$ & 3Fo-3De & $5: 2-7: 10$ & 58.41 & $2.08 \mathrm{E}-06$ & $2.00 \mathrm{E}-06$ & $2.90 \mathrm{E}+06$ \\
\hline $3 p 3 d-3 s 5 d$ & 3Fo-3De & $7: 1-7: 10$ & 58.75 & $1.62 \mathrm{E}-06$ & $2.19 \mathrm{E}-06$ & $3.12 \mathrm{E}+06$ \\
\hline $3 p 3 d-3 s 5 d$ & 3Fo-3De & $9: 1-7: 10$ & 59.15 & $3.21 \mathrm{E}-05$ & $5.62 \mathrm{E}-05$ & $7.86 \mathrm{E}+07$ \\
\hline$L S$ & 3Fo-3De & $21-15$ & & $3.15 \mathrm{E}-05$ & $1.28 \mathrm{E}-04$ & $8.52 \mathrm{E}+07$ \\
\hline $3 p 3 d-3 s 5 d$ & 1Do-3De & $5: 3-3: 13$ & 59.12 & $7.80 \mathrm{E}-06$ & $7.59 \mathrm{E}-06$ & $2.48 \mathrm{E}+07$ \\
\hline $3 p 3 d-3 s 5 d$ & 1Do-3De & $5: 3-5: 18$ & 59.12 & $7.80 \mathrm{E}-05$ & $7.59 \mathrm{E}-05$ & $1.49 \mathrm{E}+08$ \\
\hline $3 p 3 d-3 s 5 d$ & 1Do-3De & $5: 3-7: 10$ & 59.11 & $6.69 \mathrm{E}-05$ & $6.51 \mathrm{E}-05$ & $9.13 \mathrm{E}+07$ \\
\hline $3 s 4 f-3 s 5 d$ & 3Fo-3De & $5: 8-3: 13$ & 188.12 & $1.65 \mathrm{E}-02$ & $5.10 \mathrm{E}-02$ & $5.17 \mathrm{E}+09$ \\
\hline $3 s 4 f-3 s 5 d$ & 3Fo-3De & $5: 8-5: 18$ & 188.19 & $2.96 \mathrm{E}-03$ & $9.17 \mathrm{E}-03$ & $5.57 \mathrm{E}+08$ \\
\hline $3 s 4 f-3 s 5 d$ & 3Fo-3De & $7: 4-5: 18$ & 188.23 & $1.73 \mathrm{E}-02$ & $7.49 \mathrm{E}-02$ & $4.55 \mathrm{E}+09$ \\
\hline $3 s 4 f-3 s 5 d$ & 3Fo-3De & $5: 8-7: 10$ & 188.05 & $8.48 \mathrm{E}-05$ & $2.63 \mathrm{E}-04$ & $1.14 \mathrm{E}+07$ \\
\hline $3 s 4 f-3 s 5 d$ & 3Fo-3De & $7: 4-7: 10$ & 188.08 & $2.15 \mathrm{E}-03$ & $9.32 \mathrm{E}-03$ & $4.05 \mathrm{E}+08$ \\
\hline $3 s 4 f-3 s 5 d$ & 3Fo-3De & $9: 2-7: 10$ & 188.17 & $1.97 \mathrm{E}-02$ & $1.10 \mathrm{E}-01$ & $4.78 \mathrm{E}+09$ \\
\hline$L S$ & 3Fo-3De & 21-15 & & $1.96 \mathrm{E}-02$ & $2.55 \mathrm{E}-01$ & $5.17 \mathrm{E}+09$ \\
\hline $3 s 5 d-3 s 5 f$ & 3De-3Fo & $3: 13-5: 18$ & 2754.82 & $2.56 \mathrm{E}-01$ & $6.96 \mathrm{E}+00$ & $1.35 \mathrm{E}+08$ \\
\hline $3 s 5 d-3 s 5 f$ & $3 \mathrm{De}-3 \mathrm{Fo}$ & $5: 18-5: 18$ & 2739.75 & $2.81 \mathrm{E}-02$ & $1.27 \mathrm{E}+00$ & $2.50 \mathrm{E}+07$ \\
\hline $3 s 5 d-3 s 5 f$ & 3De-3Fo & $5: 18-7: 12$ & 2739.75 & $2.26 \mathrm{E}-01$ & $1.02 \mathrm{E}+01$ & $1.43 \mathrm{E}+08$ \\
\hline $3 s 5 d-3 s 5 f$ & 3De-3Fo & $7: 10-5: 18$ & 2770.15 & $5.76 \mathrm{E}-04$ & $3.68 \mathrm{E}-02$ & $7.01 \mathrm{E}+05$ \\
\hline $3 s 5 d-3 s 5 f$ & 3De-3Fo & $7: 10-7: 12$ & 2770.15 & $2.02 \mathrm{E}-02$ & $1.29 \mathrm{E}+00$ & $1.75 \mathrm{E}+07$ \\
\hline $3 s 5 d-3 s 5 f$ & $3 \mathrm{De}-3 \mathrm{Fo}$ & $7: 10-9: 5$ & 2754.82 & $2.35 \mathrm{E}-01$ & $1.49 \mathrm{E}+01$ & $1.61 \mathrm{E}+08$ \\
\hline$L S$ & 3De-3Fo & $15-21$ & & $2.55 \mathrm{E}-01$ & $3.47 \mathrm{E}+01$ & $1.61 \mathrm{E}+08$ \\
\hline $3 s 3 p-3 p^{2}$ & 3Po-1De & $3: 1-5: 1$ & 312.56 & $2.84 \mathrm{E}-02$ & $8.76 \mathrm{E}-02$ & $1.16 \mathrm{E}+09$ \\
\hline $3 \mathrm{~s} 3 \mathrm{p}-3 \mathrm{p}^{2}$ & 3Po-1De & $5: 1-5: 1$ & 327.04 & $3.29 \mathrm{E}-02$ & $1.77 \mathrm{E}-01$ & $2.05 E+09$ \\
\hline $3 s 3 p-3 s 3 d$ & 3Po-1De & $3: 1-5: 4$ & 191.41 & $3.15 \mathrm{E}-03$ & $5.95 \mathrm{E}-03$ & $3.44 \mathrm{E}+08$ \\
\hline $3 s 3 p-3 s 3 d$ & 3Po-1De & $5: 1-5: 4$ & 196.75 & $8.43 \mathrm{E}-05$ & $2.73 \mathrm{E}-04$ & $1.45 \mathrm{E}+07$ \\
\hline $3 s 3 p-3 d^{2}$ & $3 \mathrm{Po}-3 \mathrm{Fe}$ & $3: 1-5: 5$ & 88.44 & $8.37 \mathrm{E}-08$ & $7.31 \mathrm{E}-08$ & $4.28 \mathrm{E}+04$ \\
\hline $3 s 3 p-3 d^{2}$ & $3 \mathrm{Po}-3 \mathrm{Fe}$ & $5: 1-5: 5$ & 89.56 & $2.03 \mathrm{E}-08$ & $3.00 \mathrm{E}-08$ & $1.69 \mathrm{E}+04$ \\
\hline $3 s 3 p-3 d^{2}$ & $3 \mathrm{Po}-3 \mathrm{Fe}$ & $5: 1-7: 2$ & 89.43 & $2.64 \mathrm{E}-07$ & $3.88 \mathrm{E}-07$ & $1.57 \mathrm{E}+05$ \\
\hline$L S$ & $3 \mathrm{Po}-3 \mathrm{Fe}$ & $9-21$ & & $1.86 \mathrm{E}-07$ & $4.91 \mathrm{E}-07$ & $6.68 \mathrm{E}+04$ \\
\hline $3 s 3 p-3 d^{2}$ & 3Po-1De & $3: 1-5: 6$ & 85.99 & $3.38 \mathrm{E}-05$ & $2.87 \mathrm{E}-05$ & $1.83 \mathrm{E}+07$ \\
\hline $3 s 3 p-3 d^{2}$ & 3Po-1De & $5: 1-5: 6$ & 87.05 & $3.12 \mathrm{E}-06$ & $4.47 \mathrm{E}-06$ & $2.75 E+06$ \\
\hline $3 s 3 p-3 d^{2}$ & $3 \mathrm{Po}-3 \mathrm{Pe}$ & $3: 1-5: 7$ & 85.61 & $3.49 \mathrm{E}-05$ & $2.95 \mathrm{E}-05$ & $1.91 \mathrm{E}+07$ \\
\hline $3 s 3 p-3 d^{2}$ & $3 \mathrm{Po}-3 \mathrm{Pe}$ & $5: 1-5: 7$ & 86.66 & $7.88 \mathrm{E}-05$ & $1.12 \mathrm{E}-04$ & $7.00 \mathrm{E}+07$ \\
\hline$L S$ & $3 \mathrm{Po}-3 \mathrm{Pe}$ & $9-9$ & & $5.54 \mathrm{E}-05$ & $1.42 \mathrm{E}-04$ & $4.98 \mathrm{E}+07$ \\
\hline $3 s 3 p-3 s 4 d$ & 3Po-1De & $3: 1-5: 9$ & 55.69 & $3.11 \mathrm{E}-03$ & $1.71 \mathrm{E}-03$ & $4.02 \mathrm{E}+09$ \\
\hline $3 s 3 p-3 s 4 d$ & 3Po-1De & $5: 1-5: 9$ & 56.13 & $1.38 \mathrm{E}-03$ & $1.28 \mathrm{E}-03$ & $2.92 \mathrm{E}+09$ \\
\hline $3 s 3 p-3 p 4 f$ & $3 \mathrm{Po}-3 \mathrm{Fe}$ & $3: 1-5: 13$ & 46.50 & $7.38 \mathrm{E}-05$ & $3.39 \mathrm{E}-05$ & $1.37 \mathrm{E}+08$ \\
\hline $3 s 3 p-3 p 4 f$ & $3 \mathrm{Po}-3 \mathrm{Fe}$ & $5: 1-5: 13$ & 46.81 & $1.86 \mathrm{E}-05$ & $1.44 \mathrm{E}-05$ & $5.68 \mathrm{E}+07$ \\
\hline$L S$ & $3 \mathrm{Po}-3 \mathrm{Fe}$ & $9-21$ & & $3.49 \mathrm{E}-05$ & $4.83 \mathrm{E}-05$ & $4.59 \mathrm{E}+07$ \\
\hline $3 s 3 p-3 p 4 f$ & 3Po-1De & $3: 1-5: 15$ & 45.53 & $1.32 \mathrm{E}-06$ & $5.92 \mathrm{E}-07$ & $2.54 \mathrm{E}+06$ \\
\hline $3 s 3 p-3 p 4 f$ & 3Po-1De & $5: 1-5: 15$ & 45.83 & $1.43 \mathrm{E}-06$ & $1.08 \mathrm{E}-06$ & $4.55 \mathrm{E}+06$ \\
\hline $3 s 3 p-3 p^{2}$ & 1Po-1De & $3: 2-5: 1$ & 481.49 & $8.89 \mathrm{E}-02$ & $4.23 \mathrm{E}-01$ & $1.53 \mathrm{E}+09$ \\
\hline $3 \mathrm{~s} 3 \mathrm{p}-3 \mathrm{~s} 3 \mathrm{~d}$ & 1Po-1De & $3: 2-5: 4$ & 243.79 & $6.07 \mathrm{E}-01$ & $1.46 \mathrm{E}+00$ & $4.09 \mathrm{E}+10$ \\
\hline $3 \mathrm{~s} 3 \mathrm{p}-3 \mathrm{~d}^{2}$ & $1 \mathrm{Po}-3 \mathrm{Fe}$ & $3: 2-5: 5$ & 98.19 & $1.15 \mathrm{E}-07$ & $1.11 \mathrm{E}-07$ & $4.76 \mathrm{E}+04$ \\
\hline $3 s 3 p-3 d^{2}$ & 1Po-1De & $3: 2-5: 6$ & 95.18 & $1.09 \mathrm{E}-03$ & $1.02 \mathrm{E}-03$ & $4.81 \mathrm{E}+08$ \\
\hline $3 \mathrm{~s} 3 \mathrm{p}-3 \mathrm{~d}^{2}$ & $1 \mathrm{Po}-3 \mathrm{Pe}$ & $3: 2-5: 7$ & 94.71 & $1.48 \mathrm{E}-04$ & $1.39 \mathrm{E}-04$ & $6.61 \mathrm{E}+07$ \\
\hline $3 \mathrm{~s} 3 \mathrm{p}-3 \mathrm{~s} 4 \mathrm{~d}$ & 1Po-1De & $3: 2-5: 9$ & 59.40 & $2.12 \mathrm{E}-01$ & $1.24 \mathrm{E}-01$ & $2.40 \mathrm{E}+11$ \\
\hline $3 \mathrm{~s} 3 \mathrm{p}-3 \mathrm{p} 4 \mathrm{f}$ & $1 \mathrm{Po}-3 \mathrm{Fe}$ & $3: 2-5: 13$ & 49.06 & $2.93 \mathrm{E}-04$ & $1.42 \mathrm{E}-04$ & $4.87 \mathrm{E}+08$ \\
\hline $3 s 3 p-3 p 4 f$ & 1Po-1De & $3: 2-5: 15$ & 47.98 & $7.81 \mathrm{E}-03$ & $3.70 \mathrm{E}-03$ & $1.36 \mathrm{E}+10$ \\
\hline $3 p^{2}-3 p 3 d$ & 1De-3Do & $5: 1-3: 3$ & 236.25 & $1.24 \mathrm{E}-04$ & $4.83 \mathrm{E}-04$ & $2.48 \mathrm{E}+07$ \\
\hline $3 p^{2}-3 p 3 d$ & 1De-3Do & $5: 1-5: 4$ & 228.82 & $1.63 \mathrm{E}-03$ & $6.12 \mathrm{E}-03$ & $2.07 \mathrm{E}+08$ \\
\hline $3 p^{2}-3 p 3 d$ & 1De-3Do & $5: 1-7: 2$ & 229.75 & $8.16 \mathrm{E}-02$ & $3.09 \mathrm{E}-01$ & $7.37 \mathrm{E}+09$ \\
\hline $3 s 3 d-3 p 3 d$ & 1De-3Do & $5: 4-3: 3$ & 452.94 & $7.58 \mathrm{E}-04$ & $5.65 \mathrm{E}-03$ & $4.11 \mathrm{E}+07$ \\
\hline $3 s 3 d-3 p 3 d$ & 1De-3Do & $5: 4-5: 4$ & 426.38 & $1.24 \mathrm{E}-03$ & $8.68 \mathrm{E}-03$ & $4.54 \mathrm{E}+07$ \\
\hline $3 s 3 d-3 p 3 d$ & 1De-3Do & $5: 4-7: 2$ & 429.62 & $8.31 \mathrm{E}-04$ & $5.87 \mathrm{E}-03$ & $2.14 \mathrm{E}+07$ \\
\hline $3 p 3 d-3 d^{2}$ & $3 \mathrm{Do}-3 \mathrm{Fe}$ & $3: 3-5: 5$ & 258.09 & $2.39 \mathrm{E}-01$ & $6.09 \mathrm{E}-01$ & $1.43 \mathrm{E}+10$ \\
\hline $3 p 3 d-3 d^{2}$ & 3Do-3Fe & $5: 4-5: 5$ & 267.59 & $9.92 \mathrm{E}-03$ & $4.37 \mathrm{E}-02$ & $9.24 \mathrm{E}+08$ \\
\hline $3 p 3 d-3 d^{2}$ & 3Do-3Fe & $7: 2-5: 5$ & 266.33 & $2.19 \mathrm{E}-04$ & $1.35 \mathrm{E}-03$ & $2.89 \mathrm{E}+07$ \\
\hline $3 p 3 d-3 d^{2}$ & $3 \mathrm{Do}-3 \mathrm{Fe}$ & $5: 4-7: 2$ & 266.37 & $1.26 \mathrm{E}-01$ & $5.50 \mathrm{E}-01$ & $8.43 E+09$ \\
\hline $3 p 3 d-3 d^{2}$ & $3 \mathrm{Do}-3 \mathrm{Fe}$ & $7: 2-7: 2$ & 265.13 & $1.47 \mathrm{E}-02$ & $8.97 \mathrm{E}-02$ & $1.39 \mathrm{E}+09$ \\
\hline $3 p 3 d-3 d^{2}$ & 3Do-3Fe & $7: 2-9: 1$ & 263.71 & $2.86 \mathrm{E}-01$ & $1.74 \mathrm{E}+00$ & $2.13 \mathrm{E}+10$ \\
\hline$L S$ & 3Do-3Fe & $15-21$ & & $2.34 \mathrm{E}-01$ & $3.03 E+00$ & $1.61 \mathrm{E}+10$ \\
\hline $3 p 3 d-3 d^{2}$ & 3Do-1De & $3: 3-5: 6$ & 238.25 & $4.28 \mathrm{E}-03$ & $1.01 \mathrm{E}-02$ & $3.02 \mathrm{E}+08$ \\
\hline $3 p 3 d-3 d^{2}$ & 3Do-1De & $5: 4-5: 6$ & 246.32 & $5.66 \mathrm{E}-05$ & $2.30 \mathrm{E}-04$ & $6.23 \mathrm{E}+06$ \\
\hline $3 p 3 d-3 d^{2}$ & 3Do-1De & $7: 2-5: 6$ & 245.25 & $8.53 \mathrm{E}-03$ & $4.82 \mathrm{E}-02$ & $1.32 \mathrm{E}+09$ \\
\hline $3 p 3 d-3 d^{2}$ & 3Do-3Pe & $3: 3-5: 7$ & 235.35 & $1.31 \mathrm{E}-02$ & $3.06 \mathrm{E}-02$ & $9.50 \mathrm{E}+08$ \\
\hline $3 p 3 d-3 d^{2}$ & 3Do-3Pe & $5: 4-5: 7$ & 243.22 & $6.95 \mathrm{E}-02$ & $2.78 \mathrm{E}-01$ & $7.84 \mathrm{E}+09$ \\
\hline $3 p 3 d-3 d^{2}$ & 3Do-3Pe & $7: 2-5: 7$ & 242.18 & $5.76 \mathrm{E}-02$ & $3.21 \mathrm{E}-01$ & $9.17 \mathrm{E}+09$ \\
\hline$L S$ & 3Do-3Pe & $15-9$ & & $5.27 \mathrm{E}-02$ & $6.30 \mathrm{E}-01$ & $9.99 E+09$ \\
\hline $3 p 3 d-3 s 4 d$ & 3Do-1De & $3: 3-5: 9$ & 95.02 & $5.58 \mathrm{E}-06$ & $5.24 \mathrm{E}-06$ & $2.48 \mathrm{E}+06$ \\
\hline $3 p 3 d-3 s 4 d$ & 3Do-1De & $5: 4-5: 9$ & 96.28 & $8.71 \mathrm{E}-07$ & $1.38 \mathrm{E}-06$ & $6.26 \mathrm{E}+05$ \\
\hline
\end{tabular}


Table 2. (continued)

\begin{tabular}{|c|c|c|c|c|c|c|}
\hline$C_{i}-C_{k}$ & $T_{i}-T_{k}$ & $g_{i}: I-g_{j}: K$ & $E_{i k}(\AA)$ & $f$ & $S$ & $A\left(\mathrm{~s}^{-1}\right)$ \\
\hline $3 p 3 d-3 s 4 d$ & 3Do-1De & $7: 2-5: 9$ & 96.12 & $4.25 \mathrm{E}-05$ & $9.42 \mathrm{E}-05$ & $4.30 \mathrm{E}+07$ \\
\hline $3 p 3 d-3 p 4 f$ & 3Do-3Fe & $3: 3-5: 13$ & 71.06 & $7.99 \mathrm{E}-01$ & $5.61 \mathrm{E}-01$ & $6.33 \mathrm{E}+11$ \\
\hline $3 p 3 d-3 p 4 f$ & $3 \mathrm{Do}-3 \mathrm{Fe}$ & $5: 4-5: 13$ & 71.76 & $4.71 \mathrm{E}-02$ & $5.57 \mathrm{E}-02$ & $6.10 \mathrm{E}+10$ \\
\hline $3 p 3 d-3 p 4 f$ & 3Do-3Fe & $7: 2-5: 13$ & 71.67 & $1.95 \mathrm{E}-04$ & $3.22 \mathrm{E}-04$ & $3.55 E+08$ \\
\hline $3 p 3 d-3 p 4 f$ & 3Do-3Fe & $7: 2-9: 4$ & 71.06 & $5.41 \mathrm{E}-01$ & $8.87 \mathrm{E}-01$ & $5.56 \mathrm{E}+11$ \\
\hline$L S$ & 3Do-3Fe & $15-21$ & & $4.28 \mathrm{E}-01$ & $1.50 \mathrm{E}+00$ & $4.03 E+11$ \\
\hline $3 p 3 d-3 p 4 f$ & 3Do-1De & $3: 3-5: 15$ & 68.82 & $1.89 \mathrm{E}-02$ & $1.29 \mathrm{E}-02$ & $1.60 \mathrm{E}+10$ \\
\hline $3 p 3 d-3 p 4 f$ & 3Do-1De & $5: 4-5: 15$ & 69.47 & $1.04 \mathrm{E}-02$ & $1.19 \mathrm{E}-02$ & $1.44 \mathrm{E}+10$ \\
\hline $3 p 3 d-3 p 4 f$ & 3Do-1De & $7: 2-5: 15$ & 69.39 & $2.46 \mathrm{E}-04$ & $3.93 \mathrm{E}-04$ & $4.76 \mathrm{E}+08$ \\
\hline $3 p^{2}-3 p 3 d$ & $1 \mathrm{De}-3 \mathrm{Po}$ & $5: 1-3: 4$ & 229.02 & $7.43 \mathrm{E}-03$ & $2.80 \mathrm{E}-02$ & $1.58 \mathrm{E}+09$ \\
\hline $3 p^{2}-3 p 3 d$ & 1De-3Po & $5: 1-5: 5$ & 235.90 & $2.27 \mathrm{E}-02$ & $8.81 \mathrm{E}-02$ & $2.72 E+09$ \\
\hline $3 s 3 d-3 p 3 d$ & 1De-3Po & $5: 4-3: 4$ & 427.08 & $1.15 \mathrm{E}-05$ & $8.07 \mathrm{E}-05$ & $7.00 \mathrm{E}+05$ \\
\hline $3 s 3 d-3 p 3 d$ & 1De-3Po & $5: 4-5: 5$ & 451.64 & $1.24 \mathrm{E}-04$ & $9.19 \mathrm{E}-04$ & $4.04 \mathrm{E}+06$ \\
\hline $3 p 3 d-3 d^{2}$ & $3 \mathrm{Po}-3 \mathrm{Fe}$ & $3: 4-5: 5$ & 267.31 & $6.67 \mathrm{E}-02$ & $1.76 \mathrm{E}-01$ & $3.74 \mathrm{E}+09$ \\
\hline $3 p 3 d-3 d^{2}$ & $3 \mathrm{Po}-3 \mathrm{Fe}$ & $5: 5-5: 5$ & 258.52 & $1.47 \mathrm{E}-02$ & $6.24 \mathrm{E}-02$ & $1.46 \mathrm{E}+09$ \\
\hline $3 p 3 d-3 d^{2}$ & $3 \mathrm{Po}-3 \mathrm{Fe}$ & $5: 5-7: 2$ & 257.38 & $1.53 \mathrm{E}-01$ & $6.48 \mathrm{E}-01$ & $1.10 \mathrm{E}+10$ \\
\hline$L S$ & $3 \mathrm{Po}-3 \mathrm{Fe}$ & $9-21$ & & $1.15 \mathrm{E}-01$ & $8.86 \mathrm{E}-01$ & $4.91 \mathrm{E}+09$ \\
\hline $3 p 3 d-3 d^{2}$ & 3Po-1De & $3: 4-5: 6$ & 246.09 & $1.40 \mathrm{E}-02$ & $3.41 \mathrm{E}-02$ & $9.26 \mathrm{E}+08$ \\
\hline $3 p 3 d-3 d^{2}$ & 3Po-1De & $5: 5-5: 6$ & 238.61 & $8.63 \mathrm{E}-03$ & $3.39 \mathrm{E}-02$ & $1.01 \mathrm{E}+09$ \\
\hline $3 p 3 d-3 d^{2}$ & $3 \mathrm{Po}-3 \mathrm{Pe}$ & $3: 4-5: 7$ & 242.99 & $9.59 \mathrm{E}-02$ & $2.30 \mathrm{E}-01$ & $6.50 \mathrm{E}+09$ \\
\hline $3 p 3 d-3 d^{2}$ & $3 \mathrm{Po}-3 \mathrm{Pe}$ & $5: 5-5: 7$ & 235.70 & $1.68 \mathrm{E}-01$ & $6.53 \mathrm{E}-01$ & $2.02 \mathrm{E}+10$ \\
\hline$L S$ & $3 \mathrm{Po}-3 \mathrm{Pe}$ & $9-9$ & & $1.25 \mathrm{E}-01$ & $8.83 \mathrm{E}-01$ & $1.48 \mathrm{E}+10$ \\
\hline $3 p 3 d-3 s 4 d$ & 3Po-1De & $3: 4-5: 9$ & 96.24 & $2.80 \mathrm{E}-07$ & $2.66 \mathrm{E}-07$ & $1.21 \mathrm{E}+05$ \\
\hline $3 p 3 d-3 s 4 d$ & 3Po-1De & $5: 5-5: 9$ & 95.08 & $1.60 \mathrm{E}-06$ & $2.51 \mathrm{E}-06$ & $1.18 \mathrm{E}+06$ \\
\hline $3 p 3 d-3 p 4 f$ & $3 \mathrm{Po}-3 \mathrm{Fe}$ & $3: 4-5: 13$ & 71.74 & $4.46 \mathrm{E}-02$ & $3.16 \mathrm{E}-02$ & $3.47 \mathrm{E}+10$ \\
\hline $3 p 3 d-3 p 4 f$ & $3 \mathrm{Po}-3 \mathrm{Fe}$ & $5: 5-5: 13$ & 71.09 & $9.13 \mathrm{E}-03$ & $1.07 \mathrm{E}-02$ & $1.21 \mathrm{E}+10$ \\
\hline$L S$ & $3 \mathrm{Po}-3 \mathrm{Fe}$ & $9-21$ & & $1.99 \mathrm{E}-02$ & $4.23 \mathrm{E}-02$ & $1.11 \mathrm{E}+10$ \\
\hline $3 p 3 d-3 p 4 f$ & 3Po-1De & $3: 4-5: 15$ & 69.46 & $5.91 \mathrm{E}-03$ & $4.06 \mathrm{E}-03$ & $4.91 \mathrm{E}+09$ \\
\hline $3 p 3 d-3 p 4 f$ & 3Po-1De & $5: 5-5: 15$ & 68.85 & $8.15 \mathrm{E}-03$ & $9.24 \mathrm{E}-03$ & $1.15 \mathrm{E}+10$ \\
\hline $3 p^{2}-3 p 3 d$ & 1De-1Po & $5: 1-3: 5$ & 194.06 & $1.21 \mathrm{E}-03$ & $3.86 \mathrm{E}-03$ & $3.57 \mathrm{E}+08$ \\
\hline $3 s 3 d-3 p 3 d$ & $1 \mathrm{De}-1 \mathrm{Po}$ & $5: 4-3: 5$ & 319.70 & $1.11 \mathrm{E}-01$ & $5.84 \mathrm{E}-01$ & $1.21 \mathrm{E}+10$ \\
\hline $3 p 3 d-3 d^{2}$ & $1 \mathrm{Po}-3 \mathrm{Fe}$ & $3: 5-5: 5$ & 338.47 & $6.16 \mathrm{E}-04$ & $2.06 \mathrm{E}-03$ & $2.15 \mathrm{E}+07$ \\
\hline $3 p 3 d-3 d^{2}$ & 1Po-1De & $3: 5-5: 6$ & 305.15 & $1.64 \mathrm{E}-01$ & $4.95 \mathrm{E}-01$ & $7.06 \mathrm{E}+09$ \\
\hline $3 p 3 d-3 d^{2}$ & $1 \mathrm{Po}-3 \mathrm{Pe}$ & $3: 5-5: 7$ & 300.40 & $2.21 \mathrm{E}-02$ & $6.55 \mathrm{E}-02$ & $9.79 \mathrm{E}+08$ \\
\hline $3 p 3 d-3 s 4 d$ & 1Po-1De & $3: 5-5: 9$ & 104.12 & $3.78 \mathrm{E}-04$ & $3.88 \mathrm{E}-04$ & $1.39 \mathrm{E}+08$ \\
\hline $3 p 3 d-3 p 4 f$ & $1 \mathrm{Po}-3 \mathrm{Fe}$ & $3: 5-5: 13$ & 76.03 & $9.75 \mathrm{E}-03$ & $7.32 \mathrm{E}-03$ & $6.75 E+09$ \\
\hline $3 p 3 d-3 p 4 f$ & 1Po-1De & $3: 5-5: 15$ & 73.47 & $8.58 \mathrm{E}-01$ & $6.23 \mathrm{E}-01$ & $6.36 \mathrm{E}+11$ \\
\hline $3 p^{2}-3 s 4 p$ & 1De-1Po & $5: 1-3: 7$ & 75.17 & $1.74 \mathrm{E}-02$ & $2.15 \mathrm{E}-02$ & $3.42 \mathrm{E}+10$ \\
\hline $3 s 3 d-3 s 4 p$ & 1De-1Po & $5: 4-3: 7$ & 88.66 & $1.25 \mathrm{E}-02$ & $1.82 \mathrm{E}-02$ & $1.76 \mathrm{E}+10$ \\
\hline $3 d^{2}-3 s 4 p$ & $3 \mathrm{Fe}-1 \mathrm{Po}$ & $5: 5-3: 7$ & 192.44 & $1.78 \mathrm{E}-07$ & $5.64 \mathrm{E}-07$ & $5.35 \mathrm{E}+04$ \\
\hline $3 d^{2}-3 s 4 p$ & $1 \mathrm{De}-1 \mathrm{Po}$ & $5: 6-3: 7$ & 205.18 & $3.84 \mathrm{E}-05$ & $1.30 \mathrm{E}-04$ & $1.02 \mathrm{E}+07$ \\
\hline $3 d^{2}-3 s 4 p$ & $3 \mathrm{Pe}-1 \mathrm{Po}$ & $5: 7-3: 7$ & 207.38 & $5.39 \mathrm{E}-06$ & $1.84 \mathrm{E}-05$ & $1.39 \mathrm{E}+06$ \\
\hline $3 s 4 p-3 s 4 d$ & 1Po-1De & $3: 7-5: 9$ & 688.22 & $3.22 \mathrm{E}-01$ & $2.19 \mathrm{E}+00$ & $2.72 E+09$ \\
\hline $3 s 4 p-3 p 4 f$ & $1 \mathrm{Po}-3 \mathrm{Fe}$ & $3: 7-5: 13$ & 199.95 & $3.26 \mathrm{E}-07$ & $6.44 \mathrm{E}-07$ & $3.27 \mathrm{E}+04$ \\
\hline $3 s 4 p-3 p 4 f$ & 1Po-1De & $3: 7-5: 15$ & 183.14 & $4.39 \mathrm{E}-04$ & $7.94 \mathrm{E}-04$ & $5.24 \mathrm{E}+07$ \\
\hline $3 p^{2}-3 s 5 p$ & 1De-1Po & $5: 1-3: 15$ & 49.82 & $1.95 \mathrm{E}-04$ & $1.60 \mathrm{E}-04$ & $8.75 E+08$ \\
\hline $3 s 3 d-3 s 5 p$ & 1De-1Po & $5: 4-3: 15$ & 55.40 & $1.43 \mathrm{E}-04$ & $1.31 \mathrm{E}-04$ & $5.19 E+08$ \\
\hline $3 d^{2}-3 s 5 p$ & $3 \mathrm{Fe}-1 \mathrm{Po}$ & $5: 5-3: 15$ & 83.57 & $7.85 \mathrm{E}-03$ & $1.08 \mathrm{E}-02$ & $1.25 \mathrm{E}+10$ \\
\hline $3 d^{2}-3 s 5 p$ & 1De-1Po & $5: 6-3: 15$ & 85.88 & $2.14 \mathrm{E}-03$ & $3.03 \mathrm{E}-03$ & $3.23 \mathrm{E}+09$ \\
\hline $3 d^{2}-3 s 5 p$ & $3 \mathrm{Pe}-1 \mathrm{Po}$ & $5: 7-3: 15$ & 86.26 & $1.08 \mathrm{E}-05$ & $1.53 \mathrm{E}-05$ & $1.61 \mathrm{E}+07$ \\
\hline $3 s 4 d-3 s 5 p$ & 1De-1Po & $5: 9-3: 15$ & 188.07 & $5.18 \mathrm{E}-02$ & $1.60 \mathrm{E}-01$ & $1.63 E+10$ \\
\hline $3 p 4 f-3 s 5 p$ & $3 \mathrm{Fe}-1 \mathrm{Po}$ & $5: 13-3: 15$ & 565.30 & $9.86 \mathrm{E}-05$ & $9.18 \mathrm{E}-04$ & $3.43 E+06$ \\
\hline $3 p 4 f-3 s 5 p$ & 1De-1Po & $5: 15-3: 15$ & 763.40 & $1.52 \mathrm{E}-03$ & $1.91 \mathrm{E}-02$ & $2.90 \mathrm{E}+07$ \\
\hline $3 p^{2}-3 p 3 d$ & $1 \mathrm{De}-3 \mathrm{Fo}$ & $5: 1-5: 2$ & 271.26 & $2.16 \mathrm{E}-02$ & $9.66 \mathrm{E}-02$ & $1.96 \mathrm{E}+09$ \\
\hline $3 p^{2}-3 p 3 d$ & 1De-3Fo & $5: 1-7: 1$ & 264.18 & $2.16 \mathrm{E}-03$ & $9.37 \mathrm{E}-03$ & $1.47 \mathrm{E}+08$ \\
\hline $3 p^{2}-3 p 3 d$ & 1De-1Do & $5: 1-5: 3$ & 257.12 & $1.42 \mathrm{E}-01$ & $6.01 \mathrm{E}-01$ & $1.43 \mathrm{E}+10$ \\
\hline $3 p^{2}-3 s 4 f$ & 1De-3Fo & $5: 1-5: 8$ & 64.56 & $4.75 \mathrm{E}-08$ & $5.05 \mathrm{E}-08$ & $7.60 \mathrm{E}+04$ \\
\hline $3 p^{2}-3 s 4 f$ & 1De-3Fo & $5: 1-7: 4$ & 64.56 & $2.84 \mathrm{E}-05$ & $3.02 \mathrm{E}-05$ & $3.24 \mathrm{E}+07$ \\
\hline $3 p^{2}-3 s 5 f$ & 1De-3Fo & $5: 1-5: 18$ & 47.24 & $8.95 \mathrm{E}-07$ & $6.96 \mathrm{E}-07$ & $2.67 \mathrm{E}+06$ \\
\hline $3 p^{2}-3 s 5 f$ & $1 \mathrm{De}-3 \mathrm{Fo}$ & $5: 1-7: 12$ & 47.24 & $3.68 \mathrm{E}-05$ & $2.86 \mathrm{E}-05$ & $7.86 \mathrm{E}+07$ \\
\hline $3 s 3 d-3 p 3 d$ & 1De-3Fo & $5: 4-5: 2$ & 601.85 & $3.91 \mathrm{E}-03$ & $3.87 \mathrm{E}-02$ & $7.20 \mathrm{E}+07$ \\
\hline $3 s 3 d-3 p 3 d$ & 1De-3Fo & $5: 4-7: 1$ & 568.09 & $7.76 \mathrm{E}-04$ & $7.26 \mathrm{E}-03$ & $1.15 \mathrm{E}+07$ \\
\hline $3 s 3 d-3 p 3 d$ & 1De-1Do & $5: 4-5: 3$ & 536.42 & $3.15 \mathrm{E}-02$ & $2.78 \mathrm{E}-01$ & $7.31 \mathrm{E}+08$ \\
\hline $3 s 3 d-3 s 4 f$ & $1 \mathrm{De}-3 \mathrm{Fo}$ & $5: 4-5: 8$ & 74.27 & $1.81 \mathrm{E}-05$ & $2.22 \mathrm{E}-05$ & $2.19 \mathrm{E}+07$ \\
\hline $3 s 3 d-3 s 4 f$ & 1De-3Fo & $5: 4-7: 4$ & 74.26 & $1.23 \mathrm{E}-05$ & $1.51 \mathrm{E}-05$ & $1.06 \mathrm{E}+07$ \\
\hline $3 s 3 d-3 s 5 f$ & 1De-3Fo & $5: 4-5: 18$ & 52.24 & $4.25 \mathrm{E}-06$ & $3.66 \mathrm{E}-06$ & $1.04 \mathrm{E}+07$ \\
\hline $3 s 3 d-3 s 5 f$ & 1De-3Fo & $5: 4-7: 12$ & 52.24 & $1.25 \mathrm{E}-08$ & $1.07 \mathrm{E}-08$ & $2.17 \mathrm{E}+04$ \\
\hline $3 p 3 d-3 d^{2}$ & $3 \mathrm{Fo}-3 \mathrm{Fe}$ & $5: 2-5: 5$ & 226.20 & $1.17 \mathrm{E}-01$ & $4.37 \mathrm{E}-01$ & $1.53 \mathrm{E}+10$ \\
\hline $3 p 3 d-3 d^{2}$ & $3 \mathrm{Fo}-3 \mathrm{Fe}$ & $7: 1-5: 5$ & 231.37 & $1.08 \mathrm{E}-02$ & $5.75 \mathrm{E}-02$ & $1.88 \mathrm{E}+09$ \\
\hline $3 p 3 d-3 d^{2}$ & $3 \mathrm{Fo}-3 \mathrm{Fe}$ & $5: 2-7: 2$ & 225.33 & $6.89 \mathrm{E}-03$ & $2.56 \mathrm{E}-02$ & $6.47 \mathrm{E}+08$ \\
\hline $3 p 3 d-3 d^{2}$ & $3 \mathrm{Fo}-3 \mathrm{Fe}$ & $7: 1-7: 2$ & 230.46 & $1.22 \mathrm{E}-01$ & $6.46 \mathrm{E}-01$ & $1.53 \mathrm{E}+10$ \\
\hline $3 p 3 d-3 d^{2}$ & $3 \mathrm{Fo}-3 \mathrm{Fe}$ & $9: 1-7: 2$ & 236.75 & $8.11 \mathrm{E}-03$ & $5.69 \mathrm{E}-02$ & $1.24 \mathrm{E}+09$ \\
\hline $3 p 3 d-3 d^{2}$ & $3 \mathrm{Fo}-3 \mathrm{Fe}$ & $7: 1-9: 1$ & 229.39 & $2.10 \mathrm{E}-03$ & $1.11 \mathrm{E}-02$ & $2.07 \mathrm{E}+08$ \\
\hline $3 p 3 d-3 d^{2}$ & $3 \mathrm{Fo}-3 \mathrm{Fe}$ & $9: 1-9: 1$ & 235.63 & $1.22 \mathrm{E}-01$ & $8.54 \mathrm{E}-01$ & $1.47 \mathrm{E}+10$ \\
\hline$L S$ & $3 \mathrm{Fo}-3 \mathrm{Fe}$ & $21-21$ & & $1.30 \mathrm{E}-01$ & $2.09 \mathrm{E}+00$ & $1.61 \mathrm{E}+10$ \\
\hline $3 p 3 d-3 d^{2}$ & 1Do-3Fe & $5: 3-7: 2$ & 236.12 & $8.76 \mathrm{E}-05$ & $3.40 \mathrm{E}-04$ & $7.48 \mathrm{E}+06$ \\
\hline
\end{tabular}


Table 2. (continued)

\begin{tabular}{|c|c|c|c|c|c|c|}
\hline$C_{i}-C_{k}$ & $T_{i}-T_{k}$ & $g_{i}: \mathrm{I}-g_{j}: \mathrm{K}$ & $E_{i k}(\AA)$ & $f$ & $S$ & $A\left(\mathrm{~s}^{-1}\right)$ \\
\hline $3 d^{2}-3 s 4 f$ & $3 \mathrm{Fe}-3 \mathrm{Fo}$ & $5: 5-5: 8$ & 135.47 & $7.24 \mathrm{E}-05$ & $1.61 \mathrm{E}-04$ & $2.63 \mathrm{E}+07$ \\
\hline $3 d^{2}-3 s 4 f$ & $3 \mathrm{Fe}-3 \mathrm{Fo}$ & $5: 5-7: 4$ & 135.45 & $9.59 \mathrm{E}-06$ & $2.14 \mathrm{E}-05$ & $2.49 \mathrm{E}+06$ \\
\hline $3 d^{2}-3 s 4 f$ & $3 \mathrm{Fe}-3 \mathrm{Fo}$ & $7: 2-5: 8$ & 135.78 & $6.04 \mathrm{E}-06$ & $1.89 \mathrm{E}-05$ & $3.06 \mathrm{E}+06$ \\
\hline $3 d^{2}-3 s 4 f$ & $3 \mathrm{Fe}-3 \mathrm{Fo}$ & $7: 2-7: 4$ & 135.76 & $6.75 \mathrm{E}-05$ & $2.11 \mathrm{E}-04$ & $2.44 \mathrm{E}+07$ \\
\hline $3 d^{2}-3 s 4 f$ & $3 \mathrm{Fe}-3 \mathrm{Fo}$ & $7: 2-9: 2$ & 135.71 & $7.05 \mathrm{E}-06$ & $2.21 \mathrm{E}-05$ & $1.99 \mathrm{E}+06$ \\
\hline $3 d^{2}-3 s 4 f$ & $3 \mathrm{Fe}-3 \mathrm{Fo}$ & $9: 1-7: 4$ & 136.13 & $4.58 \mathrm{E}-06$ & $1.85 \mathrm{E}-05$ & $2.12 \mathrm{E}+06$ \\
\hline $3 d^{2}-3 s 4 f$ & $3 \mathrm{Fe}-3 \mathrm{Fo}$ & $9: 1-9: 2$ & 136.09 & $7.44 \mathrm{E}-05$ & $3.00 \mathrm{E}-04$ & $2.68 \mathrm{E}+07$ \\
\hline$L S$ & $3 \mathrm{Fe}-3 \mathrm{Fo}$ & $21-21$ & & $8.02 \mathrm{E}-05$ & $7.53 \mathrm{E}-04$ & $2.90 \mathrm{E}+07$ \\
\hline $3 d^{2}-3 s 5 f$ & $3 \mathrm{Fe}-3 \mathrm{Fo}$ & $5: 5-5: 18$ & 76.57 & $2.90 \mathrm{E}-04$ & $3.66 \mathrm{E}-04$ & $3.30 \mathrm{E}+08$ \\
\hline $3 d^{2}-3 s 5 f$ & $3 \mathrm{Fe}-3 \mathrm{Fo}$ & $5: 5-7: 12$ & 76.57 & $3.97 \mathrm{E}-05$ & $5.00 \mathrm{E}-05$ & $3.22 \mathrm{E}+07$ \\
\hline $3 d^{2}-3 s 5 f$ & $3 \mathrm{Fe}-3 \mathrm{Fo}$ & $7: 2-5: 18$ & 76.67 & $2.57 \mathrm{E}-05$ & $4.55 \mathrm{E}-05$ & $4.09 \mathrm{E}+07$ \\
\hline $3 d^{2}-3 s 5 f$ & $3 \mathrm{Fe}-3 \mathrm{Fo}$ & $7: 2-7: 12$ & 76.67 & $2.72 \mathrm{E}-04$ & $4.80 \mathrm{E}-04$ & $3.08 \mathrm{E}+08$ \\
\hline $3 d^{2}-3 s 5 f$ & $3 \mathrm{Fe}-3 \mathrm{Fo}$ & $7: 2-9: 5$ & 76.65 & $2.79 \mathrm{E}-05$ & $4.93 \mathrm{E}-05$ & $2.47 \mathrm{E}+07$ \\
\hline $3 d^{2}-3 s 5 f$ & $3 \mathrm{Fe}-3 \mathrm{Fo}$ & $9: 1-7: 12$ & 76.78 & $2.02 \mathrm{E}-05$ & $4.59 \mathrm{E}-05$ & $2.93 \mathrm{E}+07$ \\
\hline $3 d^{2}-3 s 5 f$ & $3 \mathrm{Fe}-3 \mathrm{Fo}$ & $9: 1-9: 5$ & 76.77 & $3.01 \mathrm{E}-04$ & $6.84 \mathrm{E}-04$ & $3.40 \mathrm{E}+08$ \\
\hline$L S$ & $3 \mathrm{Fe}-3 \mathrm{Fo}$ & $21-21$ & & $3.25 \mathrm{E}-04$ & $1.72 \mathrm{E}-03$ & $3.69 \mathrm{E}+08$ \\
\hline $3 p 3 d-3 d^{2}$ & 3Fo-1De & $5: 2-5: 6$ & 210.81 & $2.44 \mathrm{E}-02$ & $8.48 \mathrm{E}-02$ & $3.67 \mathrm{E}+09$ \\
\hline $3 p 3 d-3 d^{2}$ & 3Fo-1De & $7: 1-5: 6$ & 215.30 & $5.86 \mathrm{E}-04$ & $2.91 \mathrm{E}-03$ & $1.18 \mathrm{E}+08$ \\
\hline $3 p 3 d-3 d^{2}$ & 1Do-1De & $5: 3-5: 6$ & 220.22 & $2.03 \mathrm{E}-01$ & $7.36 \mathrm{E}-01$ & $2.79 \mathrm{E}+10$ \\
\hline $3 d^{2}-3 s 4 f$ & 1De-3Fo & $5: 6-5: 8$ & 141.66 & $3.51 \mathrm{E}-08$ & $8.19 \mathrm{E}-08$ & $1.17 \mathrm{E}+04$ \\
\hline $3 d^{2}-3 s 4 f$ & 1De-3Fo & $5: 6-7: 4$ & 141.64 & $8.05 \mathrm{E}-08$ & $1.88 \mathrm{E}-07$ & $1.91 \mathrm{E}+04$ \\
\hline $3 d^{2}-3 s 5 f$ & $1 \mathrm{De}-3 \mathrm{Fo}$ & $5: 6-5: 18$ & 78.51 & $4.00 \mathrm{E}-07$ & $5.17 \mathrm{E}-07$ & $4.33 \mathrm{E}+05$ \\
\hline $3 d^{2}-3 s 5 f$ & $1 \mathrm{De}-3 \mathrm{Fo}$ & $5: 6-7: 12$ & 78.51 & $2.67 \mathrm{E}-06$ & $3.45 \mathrm{E}-06$ & $2.07 \mathrm{E}+06$ \\
\hline $3 p 3 d-3 d^{2}$ & $3 \mathrm{Fo}-3 \mathrm{Pe}$ & $5: 2-5: 7$ & 208.54 & $6.55 \mathrm{E}-04$ & $2.25 \mathrm{E}-03$ & $1.00 \mathrm{E}+08$ \\
\hline $3 p 3 d-3 d^{2}$ & $3 \mathrm{Fo}-3 \mathrm{Pe}$ & $7: 1-5: 7$ & 212.92 & $4.69 \mathrm{E}-04$ & $2.30 \mathrm{E}-03$ & $9.66 \mathrm{E}+07$ \\
\hline$L S$ & $3 \mathrm{Fo}-3 \mathrm{Pe}$ & $21-9$ & & $3.12 \mathrm{E}-04$ & $4.55 \mathrm{E}-03$ & $1.09 \mathrm{E}+08$ \\
\hline $3 p 3 d-3 d^{2}$ & 1Do-3Pe & $5: 3-5: 7$ & 217.74 & $5.24 \mathrm{E}-03$ & $1.88 \mathrm{E}-02$ & $7.37 \mathrm{E}+08$ \\
\hline $3 d^{2}-3 s 4 f$ & $3 \mathrm{Pe}-3 \mathrm{Fo}$ & $5: 7-5: 8$ & 142.71 & $1.24 \mathrm{E}-08$ & $2.91 \mathrm{E}-08$ & $4.05 \mathrm{E}+03$ \\
\hline $3 d^{2}-3 s 4 f$ & $3 \mathrm{Pe}-3 \mathrm{Fo}$ & $5: 7-7: 4$ & 142.68 & $1.20 \mathrm{E}-08$ & $2.81 \mathrm{E}-08$ & $2.80 \mathrm{E}+03$ \\
\hline$L S$ & $3 \mathrm{Pe}-3 \mathrm{Fo}$ & $9-21$ & & $1.36 \mathrm{E}-08$ & $5.72 \mathrm{E}-08$ & $1.91 \mathrm{E}+03$ \\
\hline $3 d^{2}-3 s 5 f$ & $3 \mathrm{Pe}-3 \mathrm{Fo}$ & $5: 7-5: 18$ & 78.83 & $3.40 \mathrm{E}-08$ & $4.41 \mathrm{E}-08$ & $3.65 E+04$ \\
\hline $3 d^{2}-3 s 5 f$ & $3 \mathrm{Pe}-3 \mathrm{Fo}$ & $5: 7-7: 12$ & 78.83 & $3.44 \mathrm{E}-07$ & $4.46 \mathrm{E}-07$ & $2.64 \mathrm{E}+05$ \\
\hline$L S$ & 3Pe-3Fo & $9-21$ & & $2.10 \mathrm{E}-07$ & $4.90 \mathrm{E}-07$ & $9.68 \mathrm{E}+04$ \\
\hline $3 p 3 d-3 s 4 d$ & 3Fo-1De & $5: 2-5: 9$ & 90.33 & $1.74 \mathrm{E}-05$ & $2.59 \mathrm{E}-05$ & $1.42 \mathrm{E}+07$ \\
\hline $3 p 3 d-3 s 4 d$ & 3Fo-1De & $7: 1-5: 9$ & 91.14 & $2.26 \mathrm{E}-04$ & $4.75 \mathrm{E}-04$ & $2.54 \mathrm{E}+08$ \\
\hline $3 p 3 d-3 s 4 d$ & 1Do-1De & $5: 3-5: 9$ & 92.02 & $1.56 \mathrm{E}-06$ & $2.36 \mathrm{E}-06$ & $1.23 \mathrm{E}+06$ \\
\hline $3 s 4 d-3 s 4 f$ & 1De-3Fo & $5: 9-5: 8$ & 1365.40 & $4.34 \mathrm{E}-04$ & $9.76 \mathrm{E}-03$ & $1.55 \mathrm{E}+06$ \\
\hline $3 s 4 d-3 s 4 f$ & 1De-3Fo & $5: 9-7: 4$ & 1363.36 & $2.96 \mathrm{E}-03$ & $6.65 \mathrm{E}-02$ & $7.60 \mathrm{E}+06$ \\
\hline $3 s 4 d-3 s 5 f$ & $1 \mathrm{De}-3 \mathrm{Fo}$ & $5: 9-5: 18$ & 155.98 & $2.14 \mathrm{E}-03$ & $5.49 \mathrm{E}-03$ & $5.86 \mathrm{E}+08$ \\
\hline $3 s 4 d-3 s 5 f$ & 1De-3Fo & $5: 9-7: 12$ & 155.98 & $1.33 \mathrm{E}-02$ & $3.42 \mathrm{E}-02$ & $2.61 \mathrm{E}+09$ \\
\hline $3 p 3 d-3 p 4 f$ & 3Fo-3Fe & $5: 2-5: 13$ & 68.41 & $6.06 \mathrm{E}-02$ & $6.83 \mathrm{E}-02$ & $8.64 \mathrm{E}+10$ \\
\hline $3 p 3 d-3 p 4 f$ & $3 \mathrm{Fo}-3 \mathrm{Fe}$ & $7: 1-5: 13$ & 68.87 & $2.68 \mathrm{E}-03$ & $4.25 \mathrm{E}-03$ & $5.27 \mathrm{E}+09$ \\
\hline $3 p 3 d-3 p 4 f$ & $3 \mathrm{Fo}-3 \mathrm{Fe}$ & $7: 1-9: 4$ & 68.31 & $1.38 \mathrm{E}-01$ & $2.16 \mathrm{E}-01$ & $1.53 \mathrm{E}+11$ \\
\hline $3 p 3 d-3 p 4 f$ & 3Fo-3Fe & $9: 1-9: 4$ & 68.85 & $1.10 \mathrm{E}-01$ & $2.25 \mathrm{E}-01$ & $1.55 \mathrm{E}+11$ \\
\hline$L S$ & 3Fo-3Fe & $21-21$ & & $1.08 \mathrm{E}-01$ & $5.14 \mathrm{E}-01$ & $1.54 \mathrm{E}+11$ \\
\hline $3 p 3 d-3 p 4 f$ & 1Do-3Fe & $5: 3-5: 13$ & 69.37 & $7.15 \mathrm{E}-05$ & $8.16 \mathrm{E}-05$ & $9.91 \mathrm{E}+07$ \\
\hline $3 s 4 f-3 p 4 f$ & $3 \mathrm{Fo}-3 \mathrm{Fe}$ & $5: 8-5: 13$ & 355.13 & $8.28 \mathrm{E}-02$ & $4.84 \mathrm{E}-01$ & $4.38 \mathrm{E}+09$ \\
\hline $3 s 4 f-3 p 4 f$ & $3 \mathrm{Fo}-3 \mathrm{Fe}$ & $7: 4-5: 13$ & 355.27 & $3.18 \mathrm{E}-02$ & $2.60 \mathrm{E}-01$ & $2.35 \mathrm{E}+09$ \\
\hline $3 s 4 f-3 p 4 f$ & $3 \mathrm{Fo}-3 \mathrm{Fe}$ & $7: 4-9: 4$ & 340.75 & $6.05 \mathrm{E}-02$ & $4.75 \mathrm{E}-01$ & $2.70 \mathrm{E}+09$ \\
\hline $3 s 4 f-3 p 4 f$ & $3 \mathrm{Fo}-3 \mathrm{Fe}$ & $9: 2-9: 4$ & 341.04 & $6.66 \mathrm{E}-02$ & $6.73 \mathrm{E}-01$ & $3.82 \mathrm{E}+09$ \\
\hline$L S$ & $3 \mathrm{Fo}-3 \mathrm{Fe}$ & $21-21$ & & $7.90 \mathrm{E}-02$ & $1.89 \mathrm{E}+00$ & $4.39 \mathrm{E}+09$ \\
\hline $3 p 4 f-3 s 5 f$ & $3 \mathrm{Fe}-3 \mathrm{Fo}$ & $5: 13-5: 18$ & 349.29 & $2.38 \mathrm{E}-04$ & $1.37 \mathrm{E}-03$ & $1.30 \mathrm{E}+07$ \\
\hline $3 p 4 f-3 s 5 f$ & $3 \mathrm{Fe}-3 \mathrm{Fo}$ & $5: 13-7: 12$ & 349.29 & $3.29 \mathrm{E}-04$ & $1.89 \mathrm{E}-03$ & $1.29 \mathrm{E}+07$ \\
\hline $3 p 4 f-3 s 5 f$ & $3 \mathrm{Fe}-3 \mathrm{Fo}$ & $9: 4-7: 12$ & 364.56 & $4.71 \mathrm{E}-04$ & $5.08 \mathrm{E}-03$ & $3.04 \mathrm{E}+07$ \\
\hline $3 p 4 f-3 s 5 f$ & $3 \mathrm{Fe}-3 \mathrm{Fo}$ & $9: 4-9: 5$ & 364.29 & $4.53 \mathrm{E}-06$ & $4.88 \mathrm{E}-05$ & $2.27 \mathrm{E}+05$ \\
\hline$L S$ & $3 \mathrm{Fe}-3 \mathrm{Fo}$ & $21-21$ & & $3.39 \mathrm{E}-04$ & $8.39 \mathrm{E}-03$ & $1.76 \mathrm{E}+07$ \\
\hline $3 p 3 d-3 p 4 f$ & 3Fo-1De & $5: 2-5: 15$ & 66.32 & $3.74 \mathrm{E}-03$ & $4.08 \mathrm{E}-03$ & $5.67 \mathrm{E}+09$ \\
\hline $3 p 3 d-3 p 4 f$ & 3Fo-1De & $7: 1-5: 15$ & 66.76 & $4.28 \mathrm{E}-05$ & $6.58 \mathrm{E}-05$ & $8.96 \mathrm{E}+07$ \\
\hline $3 p 3 d-3 p 4 f$ & 1Do-1De & $5: 3-5: 15$ & 67.23 & $8.08 \mathrm{E}-02$ & $8.94 \mathrm{E}-02$ & $1.19 \mathrm{E}+11$ \\
\hline $3 s 4 f-3 p 4 f$ & 3Fo-1De & $5: 8-5: 15$ & 305.35 & $6.45 \mathrm{E}-04$ & $3.24 \mathrm{E}-03$ & $4.61 \mathrm{E}+07$ \\
\hline $3 s 4 f-3 p 4 f$ & 3Fo-1De & $7: 4-5: 15$ & 305.46 & $6.88 \mathrm{E}-03$ & $4.84 \mathrm{E}-02$ & $6.88 \mathrm{E}+08$ \\
\hline $3 p 4 f-3 s 5 f$ & $1 \mathrm{De}-3 \mathrm{Fo}$ & $5: 15-5: 18$ & 415.98 & $4.65 \mathrm{E}-05$ & $3.19 \mathrm{E}-04$ & $1.79 \mathrm{E}+06$ \\
\hline $3 p 4 f-3 s 5 f$ & $1 \mathrm{De}-3 \mathrm{Fo}$ & $5: 15-7: 12$ & 415.98 & $1.66 \mathrm{E}-04$ & $1.14 \mathrm{E}-03$ & $4.57 \mathrm{E}+06$ \\
\hline $3 p^{2}-3 p 3 d$ & $1 \mathrm{De}-1 \mathrm{Fo}$ & $5: 1-7: 3$ & 198.84 & $1.72 \mathrm{E}-01$ & $5.62 \mathrm{E}-01$ & $2.07 \mathrm{E}+10$ \\
\hline $3 p^{2}-3 s 4 f$ & 1De-1Fo & $5: 1-7: 5$ & 63.96 & $1.64 \mathrm{E}-01$ & $1.72 \mathrm{E}-01$ & $1.91 \mathrm{E}+11$ \\
\hline $3 p^{2}-3 s 5 f ! 3 p 5 d^{3}$ & $1 \mathrm{De}-1 \mathrm{Fo}$ & $5: 1-7: 14$ & 44.98 & $4.51 \mathrm{E}-07$ & $3.34 \mathrm{E}-07$ & $1.06 \mathrm{E}+06$ \\
\hline $3 p^{2}-3 s 6 f$ & 1De-1Fo & $5: 1-7: 25$ & 39.50 & $1.88 \mathrm{E}-05$ & $1.22 \mathrm{E}-05$ & $5.74 \mathrm{E}+07$ \\
\hline $3 p^{2}-3 p 3 d$ & $3 \mathrm{Pe}-1 \mathrm{Fo}$ & $5: 2-7: 3$ & 208.03 & $4.96 \mathrm{E}-02$ & $1.70 \mathrm{E}-01$ & $5.46 \mathrm{E}+09$ \\
\hline $3 p^{2}-3 s 4 f$ & $3 \mathrm{Pe}-1 \mathrm{Fo}$ & $5: 2-7: 5$ & 64.88 & $4.08 \mathrm{E}-02$ & $4.36 \mathrm{E}-02$ & $4.62 \mathrm{E}+10$ \\
\hline $3 p^{2}-3 s 5 f ! 3 p 5 d^{3}$ & $3 \mathrm{Pe}-1 \mathrm{Fo}$ & $5: 2-7: 14$ & 45.44 & $3.10 \mathrm{E}-06$ & $2.32 \mathrm{E}-06$ & $7.15 E+06$ \\
\hline $3 p^{2}-3 s 6 f$ & $3 \mathrm{Pe}-1 \mathrm{Fo}$ & $5: 2-7: 25$ & 39.85 & $6.70 \mathrm{E}-07$ & $4.39 \mathrm{E}-07$ & $2.01 \mathrm{E}+06$ \\
\hline $3 s 3 d-3 p 3 d$ & 3De-1Fo & $5: 3-7: 3$ & 261.29 & $3.79 \mathrm{E}-04$ & $1.63 \mathrm{E}-03$ & $2.64 \mathrm{E}+07$ \\
\hline $3 s 3 d-3 p 3 d$ & 3De-1Fo & $7: 1-7: 3$ & 262.40 & $1.40 \mathrm{E}-03$ & $8.49 \mathrm{E}-03$ & $1.36 \mathrm{E}+08$ \\
\hline $3 s 3 d-3 s 4 f$ & 3De-1Fo & $5: 3-7: 5$ & 69.28 & $5.02 \mathrm{E}-07$ & $5.73 \mathrm{E}-07$ & $4.99 \mathrm{E}+05$ \\
\hline $3 s 3 d-3 s 4 f$ & 3De-1Fo & $7: 1-7: 5$ & 69.36 & $5.17 \mathrm{E}-06$ & $8.27 \mathrm{E}-06$ & $7.17 \mathrm{E}+06$ \\
\hline $3 s 3 d-3 s 5 f ! 3 p 5 d^{3}$ & 3De-1Fo & $5: 3-7: 14$ & 47.55 & $6.72 \mathrm{E}-05$ & $5.26 \mathrm{E}-05$ & $1.42 \mathrm{E}+08$ \\
\hline
\end{tabular}


Table 2. (continued)

\begin{tabular}{|c|c|c|c|c|c|c|}
\hline$C_{i}-C_{k}$ & $T_{i}-T_{k}$ & $g_{i}: \mathrm{I}-g_{j}: \mathrm{K}$ & $E_{i k}(\AA)$ & $f$ & $S$ & $A\left(\mathrm{~s}^{-1}\right)$ \\
\hline $3 s 3 d-3 s 5 f ! 3 p 5 d^{3}$ & 3De-1Fo & $7: 1-7: 14$ & 47.59 & $2.22 \mathrm{E}-07$ & $2.44 \mathrm{E}-07$ & $6.55 \mathrm{E}+05$ \\
\hline $3 s 3 d-3 s 6 f$ & 3De-1Fo & $5: 3-7: 25$ & 41.46 & $5.50 \mathrm{E}-04$ & $3.75 \mathrm{E}-04$ & $1.52 \mathrm{E}+09$ \\
\hline $3 s 3 d-3 s 6 f$ & 3De-1Fo & $7: 1-7: 25$ & 41.49 & $4.69 \mathrm{E}-05$ & $4.48 \mathrm{E}-05$ & $1.82 \mathrm{E}+08$ \\
\hline $3 s 3 d-3 p 3 d$ & 1De-1Fo & $5: 4-7: 3$ & 332.87 & $3.90 \mathrm{E}-01$ & $2.14 \mathrm{E}+00$ & $1.68 \mathrm{E}+10$ \\
\hline $3 s 3 d-3 s 4 f$ & 1De-1Fo & $5: 4-7: 5$ & 73.47 & $6.89 \mathrm{E}-01$ & $8.33 \mathrm{E}-01$ & $6.08 \mathrm{E}+11$ \\
\hline $3 s 3 d-3 s 5 f ! 3 p 5 d^{3}$ & 1De-1Fo & $5: 4-7: 14$ & 49.49 & $5.74 \mathrm{E}-06$ & $4.68 \mathrm{E}-06$ & $1.12 \mathrm{E}+07$ \\
\hline $3 s 3 d-3 s 6 f$ & 1De-1Fo & $5: 4-7: 25$ & 42.93 & $8.52 \mathrm{E}-04$ & $6.02 \mathrm{E}-04$ & $2.20 \mathrm{E}+09$ \\
\hline $3 p 3 d-3 d^{2}$ & 1Fo-3Fe & $7: 3-5: 5$ & 324.86 & $1.93 \mathrm{E}-04$ & $1.45 \mathrm{E}-03$ & $1.71 \mathrm{E}+07$ \\
\hline $3 p 3 d-3 d^{2}$ & 1Fo-3Fe & $7: 3-7: 2$ & 323.08 & $5.48 \mathrm{E}-04$ & $4.08 \mathrm{E}-03$ & $3.50 \mathrm{E}+07$ \\
\hline $3 p 3 d-3 d^{2}$ & $1 \mathrm{Fo}-3 \mathrm{Fe}$ & $7: 3-9: 1$ & 320.98 & $2.51 \mathrm{E}-04$ & $1.86 \mathrm{E}-03$ & $1.26 \mathrm{E}+07$ \\
\hline $3 d^{2}-3 s 4 f$ & $3 \mathrm{Fe}-1 \mathrm{Fo}$ & $5: 5-7: 5$ & 132.83 & $7.82 \mathrm{E}-07$ & $1.71 \mathrm{E}-06$ & $2.11 \mathrm{E}+05$ \\
\hline $3 d^{2}-3 s 4 f$ & $3 \mathrm{Fe}-1 \mathrm{Fo}$ & $7: 2-7: 5$ & 133.13 & $1.41 \mathrm{E}-07$ & $4.32 \mathrm{E}-07$ & $5.30 \mathrm{E}+04$ \\
\hline $3 d^{2}-3 s 4 f$ & 3Fe-1Fo & $9: 1-7: 5$ & 133.49 & $1.83 \mathrm{E}-08$ & $7.24 \mathrm{E}-08$ & $8.81 E+03$ \\
\hline $3 d^{2}-3 s 5 f ! 3 p 5 d^{3}$ & $3 \mathrm{Fe}-1 \mathrm{Fo}$ & $5: 5-7: 14$ & 70.80 & $6.29 \mathrm{E}-02$ & $7.33 \mathrm{E}-02$ & $5.98 \mathrm{E}+10$ \\
\hline $3 d^{2}-3 s 5 f ! 3 p 5 d^{3}$ & $3 \mathrm{Fe}-1 \mathrm{Fo}$ & $7: 2-7: 14$ & 70.89 & $3.19 \mathrm{E}-01$ & $5.20 \mathrm{E}-01$ & $4.23 E+11$ \\
\hline $3 d^{2}-3 s 5 f ! 3 p 5 d^{3}$ & $3 \mathrm{Fe}-1 \mathrm{Fo}$ & $9: 1-7: 14$ & 70.99 & $2.24 \mathrm{E}-02$ & $4.72 \mathrm{E}-02$ & $3.82 \mathrm{E}+10$ \\
\hline $3 d^{2}-3 s 6 f$ & $3 \mathrm{Fe}-1 \mathrm{Fo}$ & $5: 5-7: 25$ & 58.10 & $7.77 \mathrm{E}-05$ & $7.43 \mathrm{E}-05$ & $1.10 \mathrm{E}+08$ \\
\hline $3 d^{2}-3 s 6 f$ & $3 \mathrm{Fe}-1 \mathrm{Fo}$ & $7: 2-7: 25$ & 58.16 & $1.26 \mathrm{E}-04$ & $1.68 \mathrm{E}-04$ & $2.48 \mathrm{E}+08$ \\
\hline $3 d^{2}-3 s 6 f$ & $3 \mathrm{Fe}-1 \mathrm{Fo}$ & $9: 1-7: 25$ & 58.23 & $1.02 \mathrm{E}-05$ & $1.76 \mathrm{E}-05$ & $2.59 \mathrm{E}+07$ \\
\hline $3 p 3 d-3 d^{2}$ & 1Fo-1De & $7: 3-5: 6$ & 294.04 & $2.47 \mathrm{E}-02$ & $1.67 \mathrm{E}-01$ & $2.66 \mathrm{E}+09$ \\
\hline $3 d^{2}-3 s 4 f$ & 1De-1Fo & $5: 6-7: 5$ & 138.78 & $1.97 \mathrm{E}-04$ & $4.49 \mathrm{E}-04$ & $4.86 \mathrm{E}+07$ \\
\hline $3 d^{2}-3 s 5 f ! 3 p 5 d^{3}$ & 1De-1Fo & $5: 6-7: 14$ & 72.46 & $1.20 \mathrm{E}-03$ & $1.43 \mathrm{E}-03$ & $1.09 E+09$ \\
\hline $3 d^{2}-3 s 6 f$ & 1De-1Fo & $5: 6-7: 25$ & 59.21 & $2.64 \mathrm{E}-03$ & $2.57 \mathrm{E}-03$ & $3.58 \mathrm{E}+09$ \\
\hline $3 p 3 d-3 d^{2}$ & $1 \mathrm{Fo}-3 \mathrm{Pe}$ & $7: 3-5: 7$ & 289.63 & $5.18 \mathrm{E}-03$ & $3.46 \mathrm{E}-02$ & $5.76 \mathrm{E}+08$ \\
\hline $3 d^{2}-3 s 4 f$ & 3Pe-1Fo & $5: 7-7: 5$ & 139.79 & $2.22 \mathrm{E}-05$ & $5.11 \mathrm{E}-05$ & $5.41 \mathrm{E}+06$ \\
\hline $3 d^{2}-3 s 5 f ! 3 p 5 d^{3}$ & $3 \mathrm{Pe}-1 \mathrm{Fo}$ & $5: 7-7: 14$ & 72.73 & $7.21 \mathrm{E}-04$ & $8.63 \mathrm{E}-04$ & $6.49 \mathrm{E}+08$ \\
\hline $3 d^{2}-3 s 6 f$ & $3 \mathrm{Pe}-1 \mathrm{Fo}$ & $5: 7-7: 25$ & 59.39 & $3.02 \mathrm{E}-04$ & $2.95 \mathrm{E}-04$ & $4.08 \mathrm{E}+08$ \\
\hline $3 p 3 d-3 s 4 d$ & 1Fo-3De & $7: 3-5: 8$ & 103.15 & $2.65 \mathrm{E}-04$ & $6.30 \mathrm{E}-04$ & $2.32 \mathrm{E}+08$ \\
\hline $3 p 3 d-3 s 4 d$ & 1Fo-3De & $7: 3-7: 3$ & 103.02 & $1.17 \mathrm{E}-06$ & $2.79 \mathrm{E}-06$ & $7.37 \mathrm{E}+05$ \\
\hline $3 s 4 d-3 s 4 f$ & $3 \mathrm{De}-1 \mathrm{Fo}$ & $5: 8-7: 5$ & 1097.25 & $4.33 \mathrm{E}-03$ & $7.81 \mathrm{E}-02$ & $1.71 \mathrm{E}+07$ \\
\hline $3 s 4 d-3 s 4 f$ & 3De-1Fo & $7: 3-7: 5$ & 1111.44 & $5.42 \mathrm{E}-07$ & $1.39 \mathrm{E}-05$ & $2.92 E+03$ \\
\hline $3 s 4 d-3 s 5 f ! 3 p 5 d^{3}$ & 3De-1Fo & $5: 8-7: 14$ & 133.21 & $2.54 \mathrm{E}-04$ & $5.56 \mathrm{E}-04$ & $6.81 E+07$ \\
\hline $3 s 4 d-3 s 5 f ! 3 p 5 d^{3}$ & 3De-1Fo & $7: 3-7: 14$ & 133.42 & $3.18 \mathrm{E}-05$ & $9.78 \mathrm{E}-05$ & $1.19 \mathrm{E}+07$ \\
\hline $3 s 4 d-3 s 6 f$ & 3De-1Fo & $5: 8-7: 25$ & 94.39 & $1.23 \mathrm{E}-03$ & $1.91 \mathrm{E}-03$ & $6.58 \mathrm{E}+08$ \\
\hline $3 s 4 d-3 s 6 f$ & 3De-1Fo & $7: 3-7: 25$ & 94.49 & $4.65 \mathrm{E}-05$ & $1.01 \mathrm{E}-04$ & $3.47 \mathrm{E}+07$ \\
\hline $3 p 3 d-3 s 4 d$ & 1Fo-1De & $7: 3-5: 9$ & 102.80 & $6.76 \mathrm{E}-03$ & $1.60 \mathrm{E}-02$ & $5.97 \mathrm{E}+09$ \\
\hline $3 s 4 d-3 s 4 f$ & 1De-1Fo & $5: 9-7: 5$ & 1137.95 & $1.54 \mathrm{E}-01$ & $2.88 \mathrm{E}+00$ & $5.66 \mathrm{E}+08$ \\
\hline $3 s 4 d-3 s 5 f ! 3 p 5 d^{3}$ & 1De-1Fo & $5: 9-7: 14$ & 133.79 & $1.51 \mathrm{E}-05$ & $3.33 \mathrm{E}-05$ & $4.03 E+06$ \\
\hline $3 s 4 d-3 s 6 f$ & 1De-1Fo & $5: 9-7: 25$ & 94.68 & $4.47 \mathrm{E}-03$ & $6.97 \mathrm{E}-03$ & $2.38 \mathrm{E}+09$ \\
\hline $3 p 3 d-3 p 4 f$ & $1 \mathrm{Fo}-3 \mathrm{Fe}$ & $7: 3-5: 13$ & 75.32 & $8.68 \mathrm{E}-05$ & $1.51 \mathrm{E}-04$ & $1.43 E+08$ \\
\hline $3 p 3 d-3 p 4 f$ & $1 \mathrm{Fo}-3 \mathrm{Fe}$ & $7: 3-9: 4$ & 74.65 & $1.15 \mathrm{E}-04$ & $1.97 \mathrm{E}-04$ & $1.07 \mathrm{E}+08$ \\
\hline $3 s 4 f-3 p 4 f$ & 1Fo-3Fe & $7: 5-5: 13$ & 374.61 & $3.80 \mathrm{E}-03$ & $3.28 \mathrm{E}-02$ & $2.53 \mathrm{E}+08$ \\
\hline $3 s 4 f-3 p 4 f$ & $1 \mathrm{Fo}-3 \mathrm{Fe}$ & $7: 5-9: 4$ & 358.50 & $2.29 \mathrm{E}-05$ & $1.89 \mathrm{E}-04$ & $9.24 \mathrm{E}+05$ \\
\hline $3 p 4 f-3 s 5 f ! 3 p 5 d^{3}$ & 3Fe-1Fo & $5: 13-7: 14$ & 254.71 & $2.43 \mathrm{E}-03$ & $1.02 \mathrm{E}-02$ & $1.78 \mathrm{E}+08$ \\
\hline $3 p 4 f-3 s 5 f ! 3 p 5 d^{3}$ & 3Fe-1Fo & $9: 4-7: 14$ & 262.74 & $2.22 \mathrm{E}-05$ & $1.73 \mathrm{E}-04$ & $2.75 E+06$ \\
\hline $3 p 4 f-3 s 6 f$ & $3 \mathrm{Fe}-1 \mathrm{Fo}$ & $5: 13-7: 25$ & 142.57 & $2.67 \mathrm{E}-02$ & $6.26 \mathrm{E}-02$ & $6.25 E+09$ \\
\hline $3 p 4 f-3 s 6 f$ & $3 \mathrm{Fe}-1 \mathrm{Fo}$ & $9: 4-7: 25$ & 145.05 & $1.16 \mathrm{E}-03$ & $4.98 \mathrm{E}-03$ & $4.72 \mathrm{E}+08$ \\
\hline $3 p 3 d-3 p 4 f$ & 1Fo-3De & $7: 3-5: 14$ & 73.98 & $2.90 \mathrm{E}-05$ & $4.94 \mathrm{E}-05$ & $4.94 \mathrm{E}+07$ \\
\hline $3 p 3 d-3 p 4 f$ & 1Fo-3De & $7: 3-7: 8$ & 74.05 & $7.21 \mathrm{E}-03$ & $1.23 \mathrm{E}-02$ & $8.77 \mathrm{E}+09$ \\
\hline $3 s 4 f-3 p 4 f$ & 1Fo-3De & $7: 5-5: 14$ & 343.47 & $2.61 \mathrm{E}-03$ & $2.07 \mathrm{E}-02$ & $2.07 \mathrm{E}+08$ \\
\hline $3 s 4 f-3 p 4 f$ & 1Fo-3De & $7: 5-7: 8$ & 345.01 & $6.17 \mathrm{E}-03$ & $4.91 \mathrm{E}-02$ & $3.46 \mathrm{E}+08$ \\
\hline $3 p 4 f-3 s 5 f ! 3 p 5 d^{3}$ & 3De-1Fo & $5: 14-7: 14$ & 271.44 & $3.72 \mathrm{E}-02$ & $1.66 \mathrm{E}-01$ & $2.40 \mathrm{E}+09$ \\
\hline $3 p 4 f-3 s 5 f ! 3 p 5 d^{3}$ & 3De-1Fo & $7: 8-7: 14$ & 270.49 & $2.73 \mathrm{E}-03$ & $1.70 \mathrm{E}-02$ & $2.49 \mathrm{E}+08$ \\
\hline $3 p 4 f-3 s 6 f$ & $3 \mathrm{De}-1 \mathrm{Fo}$ & $5: 14-7: 25$ & 147.67 & $1.95 \mathrm{E}-01$ & $4.74 \mathrm{E}-01$ & $4.26 \mathrm{E}+10$ \\
\hline $3 p 4 f-3 s 6 f$ & 3De-1Fo & $7: 8-7: 25$ & 147.38 & $8.04 \mathrm{E}-02$ & $2.73 \mathrm{E}-01$ & $2.47 \mathrm{E}+10$ \\
\hline $3 p 3 d-3 p 4 f$ & 1Fo-1De & $7: 3-5: 15$ & 72.81 & $2.87 \mathrm{E}-03$ & $4.82 \mathrm{E}-03$ & $5.06 \mathrm{E}+09$ \\
\hline $3 s 4 f-3 p 4 f$ & 1Fo-1De & $7: 5-5: 15$ & 319.64 & $1.03 \mathrm{E}-01$ & $7.61 \mathrm{E}-01$ & $9.44 \mathrm{E}+09$ \\
\hline $3 p 4 f-3 s 5 f ! 3 p 5 d^{3}$ & 1De-1Fo & $5: 15-7: 14$ & 288.44 & $6.53 \mathrm{E}-04$ & $3.10 \mathrm{E}-03$ & $3.74 \mathrm{E}+07$ \\
\hline $3 p 4 f-3 s 6 f$ & 1De-1Fo & $5: 15-7: 25$ & 152.56 & $1.27 \mathrm{E}+00$ & $3.18 \mathrm{E}+00$ & $2.59 \mathrm{E}+11$ \\
\hline $3 p 3 d-3 s 5 d$ & 1Fo-3De & $7: 3-5: 18$ & 63.40 & $2.59 \mathrm{E}-09$ & $3.79 \mathrm{E}-09$ & $6.03 E+03$ \\
\hline $3 p 3 d-3 s 5 d$ & 1Fo-3De & $7: 3-7: 10$ & 63.38 & $1.29 \mathrm{E}-07$ & $1.89 \mathrm{E}-07$ & $2.15 \mathrm{E}+05$ \\
\hline $3 s 4 f-3 s 5 d$ & 1Fo-3De & $7: 5-5: 18$ & 193.52 & $3.00 \mathrm{E}-04$ & $1.34 \mathrm{E}-03$ & $7.48 \mathrm{E}+07$ \\
\hline $3 s 4 f-3 s 5 d$ & 1Fo-3De & $7: 5-7: 10$ & 193.37 & $9.27 \mathrm{E}-08$ & $4.13 \mathrm{E}-07$ & $1.65 E+04$ \\
\hline $3 s 5 d-3 s 5 f ! 3 p 5 d^{3}$ & $3 \mathrm{De}-1 \mathrm{Fo}$ & $5: 18-7: 14$ & 700.28 & $3.90 \mathrm{E}-07$ & $4.49 \mathrm{E}-06$ & $3.79 \mathrm{E}+03$ \\
\hline $3 s 5 d-3 s 5 f ! 3 p 5 d^{3}$ & 3De-1Fo & $7: 10-7: 14$ & 702.25 & $5.88 \mathrm{E}-08$ & $9.52 \mathrm{E}-07$ & $7.95 \mathrm{E}+02$ \\
\hline $3 s 5 d-3 s 6 f$ & 3De-1Fo & $5: 18-7: 25$ & 221.44 & $1.44 \mathrm{E}-02$ & $5.25 \mathrm{E}-02$ & $1.40 \mathrm{E}+09$ \\
\hline $3 s 5 d-3 s 6 f$ & 3De-1Fo & $7: 10-7: 25$ & 221.63 & $1.19 \mathrm{E}-03$ & $6.07 \mathrm{E}-03$ & $1.61 \mathrm{E}+08$ \\
\hline $3 s 3 p-3 p 4 f$ & $3 \mathrm{Po}-3 \mathrm{Ge}$ & $5: 1-7: 5$ & 47.03 & $6.99 \mathrm{E}-06$ & $5.41 \mathrm{E}-06$ & $1.51 \mathrm{E}+07$ \\
\hline $3 s 3 p-3 p 4 f$ & $3 \mathrm{Po}-1 \mathrm{Fe}$ & $5: 1-7: 6$ & 46.89 & $1.08 \mathrm{E}-04$ & $8.36 \mathrm{E}-05$ & $2.35 \mathrm{E}+08$ \\
\hline $3 p 3 d-3 p 4 f$ & $3 \mathrm{Fo}-3 \mathrm{Ge}$ & $5: 2-7: 5$ & 68.87 & $7.68 \mathrm{E}-01$ & $8.71 \mathrm{E}-01$ & $7.71 \mathrm{E}+11$ \\
\hline $3 p 3 d-3 p 4 f$ & $3 \mathrm{Fo}-3 \mathrm{Ge}$ & $7: 1-7: 5$ & 69.35 & $2.37 \mathrm{E}-02$ & $3.79 \mathrm{E}-02$ & $3.29 \mathrm{E}+10$ \\
\hline $3 p 3 d-3 p 4 f$ & $3 \mathrm{Fo}-3 \mathrm{Ge}$ & $9: 1-7: 5$ & 69.91 & $5.00 \mathrm{E}-06$ & $1.04 \mathrm{E}-05$ & $8.77 \mathrm{E}+06$ \\
\hline $3 p 3 d-3 p 4 f$ & $3 \mathrm{Fo}-3 \mathrm{Ge}$ & $7: 1-9: 3$ & 69.03 & $6.52 \mathrm{E}-01$ & $1.04 \mathrm{E}+00$ & $7.10 \mathrm{E}+11$ \\
\hline $3 p 3 d-3 p 4 f$ & $3 \mathrm{Fo}-3 \mathrm{Ge}$ & $9: 1-9: 3$ & 69.59 & $2.93 \mathrm{E}-04$ & $6.04 \mathrm{E}-04$ & $4.04 \mathrm{E}+08$ \\
\hline $3 p 3 d-3 p 4 f$ & $3 \mathrm{Fo}-3 \mathrm{Ge}$ & $9: 1-11: 1$ & 68.85 & $8.01 \mathrm{E}-01$ & $1.63 \mathrm{E}+00$ & $9.22 \mathrm{E}+11$ \\
\hline$L S$ & $3 \mathrm{Fo}-3 \mathrm{Ge}$ & $21-27$ & & $7.52 \mathrm{E}-01$ & $3.58 \mathrm{E}+00$ & $8.21 \mathrm{E}+11$ \\
\hline
\end{tabular}


Table 2. (continued)

\begin{tabular}{|c|c|c|c|c|c|c|}
\hline$C_{i}-C_{k}$ & $T_{i}-T_{k}$ & $g_{i}: \mathrm{I}-g_{j}: \mathrm{K}$ & $E_{i k}(\AA)$ & $f$ & $S$ & $A\left(\mathrm{~s}^{-1}\right)$ \\
\hline $3 p 3 d-3 p 4 f$ & $3 \mathrm{Fo}-1 \mathrm{Fe}$ & $5: 2-7: 6$ & 68.57 & $4.14 \mathrm{E}-02$ & $4.68 \mathrm{E}-02$ & $4.20 \mathrm{E}+10$ \\
\hline $3 p 3 d-3 p 4 f$ & $3 \mathrm{Fo}-1 \mathrm{Fe}$ & $7: 1-7: 6$ & 69.03 & $7.72 \mathrm{E}-03$ & $1.23 \mathrm{E}-02$ & $1.08 \mathrm{E}+10$ \\
\hline $3 p 3 d-3 p 4 f$ & $3 \mathrm{Fo}-1 \mathrm{Fe}$ & $9: 1-7: 6$ & 69.59 & $6.46 \mathrm{E}-05$ & $1.33 \mathrm{E}-04$ & $1.14 \mathrm{E}+08$ \\
\hline $3 p 3 d-3 p 4 f$ & 1Do-3Ge & $5: 3-7: 5$ & 69.85 & $6.31 \mathrm{E}-02$ & $7.25 \mathrm{E}-02$ & $6.16 \mathrm{E}+10$ \\
\hline $3 p 3 d-3 p 4 f$ & 1Do-1Fe & $5: 3-7: 6$ & 69.53 & $5.14 \mathrm{E}-01$ & $5.89 \mathrm{E}-01$ & $5.07 E+11$ \\
\hline $3 p 3 d-3 p 4 f$ & 3Do-3Ge & $5: 4-7: 5$ & 72.28 & $3.44 \mathrm{E}-03$ & $4.10 \mathrm{E}-03$ & $3.14 \mathrm{E}+09$ \\
\hline $3 p 3 d-3 p 4 f$ & 3Do-3Ge & $7: 2-7: 5$ & 72.19 & $4.41 \mathrm{E}-03$ & $7.34 \mathrm{E}-03$ & $5.65 E+09$ \\
\hline $3 p 3 d-3 p 4 f$ & 3Do-3Ge & $7: 2-9: 3$ & 71.85 & $1.60 \mathrm{E}-01$ & $2.65 \mathrm{E}-01$ & $1.61 \mathrm{E}+11$ \\
\hline$L S$ & 3Do-3Ge & $15-27$ & & $7.79 \mathrm{E}-02$ & $2.76 \mathrm{E}-01$ & $5.58 \mathrm{E}+10$ \\
\hline $3 p 3 d-3 p 4 f$ & $3 \mathrm{Do}-1 \mathrm{Fe}$ & $5: 4-7: 6$ & 71.94 & $2.52 \mathrm{E}-01$ & $2.98 \mathrm{E}-01$ & $2.32 \mathrm{E}+11$ \\
\hline $3 p 3 d-3 p 4 f$ & 3Do-1Fe & $7: 2-7: 6$ & 71.85 & $1.72 \mathrm{E}-03$ & $2.85 \mathrm{E}-03$ & $2.23 E+09$ \\
\hline $3 p 3 d-3 p 4 f$ & $3 \mathrm{Po}-3 \mathrm{Ge}$ & $5: 5-7: 5$ & 71.60 & $6.14 \mathrm{E}-03$ & $7.24 \mathrm{E}-03$ & $5.71 \mathrm{E}+09$ \\
\hline $3 p 3 d-3 p 4 f$ & $3 \mathrm{Po}-1 \mathrm{Fe}$ & $5: 5-7: 6$ & 71.27 & $4.87 \mathrm{E}-02$ & $5.71 \mathrm{E}-02$ & $4.57 \mathrm{E}+10$ \\
\hline $3 s 4 f-3 p 4 f$ & $3 \mathrm{Fo}-3 \mathrm{Ge}$ & $5: 8-7: 5$ & 368.13 & $3.65 \mathrm{E}-02$ & $2.21 \mathrm{E}-01$ & $1.28 \mathrm{E}+09$ \\
\hline $3 s 4 f-3 p 4 f$ & $3 \mathrm{Fo}-3 \mathrm{Ge}$ & $7: 4-7: 5$ & 368.28 & $1.39 \mathrm{E}-02$ & $1.18 \mathrm{E}-01$ & $6.84 \mathrm{E}+08$ \\
\hline $3 s 4 f-3 p 4 f$ & $3 \mathrm{Fo}-3 \mathrm{Ge}$ & $9: 2-7: 5$ & 368.62 & $7.62 \mathrm{E}-05$ & $8.33 \mathrm{E}-04$ & $4.81 \mathrm{E}+06$ \\
\hline $3 s 4 f-3 p 4 f$ & $3 \mathrm{Fo}-3 \mathrm{Ge}$ & $7: 4-9: 3$ & 359.62 & $2.55 \mathrm{E}-02$ & $2.12 \mathrm{E}-01$ & $1.02 \mathrm{E}+09$ \\
\hline $3 s 4 f-3 p 4 f$ & $3 \mathrm{Fo}-3 \mathrm{Ge}$ & $9: 2-9: 3$ & 359.94 & $5.87 \mathrm{E}-02$ & $6.26 \mathrm{E}-01$ & $3.02 E+09$ \\
\hline $3 s 4 f-3 p 4 f$ & $3 \mathrm{Fo}-3 \mathrm{Ge}$ & $9: 2-11: 1$ & 341.04 & $7.89 \mathrm{E}-02$ & $7.97 \mathrm{E}-01$ & $3.70 \mathrm{E}+09$ \\
\hline$L S$ & $3 \mathrm{Fo}-3 \mathrm{Ge}$ & $21-27$ & & $8.08 \mathrm{E}-02$ & $1.97 \mathrm{E}+00$ & $3.36 \mathrm{E}+09$ \\
\hline $3 s 4 f-3 p 4 f$ & $3 \mathrm{Fo}-1 \mathrm{Fe}$ & $5: 8-7: 6$ & 359.47 & $1.00 \mathrm{E}-03$ & $5.93 \mathrm{E}-03$ & $3.69 \mathrm{E}+07$ \\
\hline $3 s 4 f-3 p 4 f$ & $3 \mathrm{Fo}-1 \mathrm{Fe}$ & $7: 4-7: 6$ & 359.62 & $3.79 \mathrm{E}-02$ & $3.14 \mathrm{E}-01$ & $1.96 E+09$ \\
\hline $3 s 4 f-3 p 4 f$ & $3 \mathrm{Fo}-1 \mathrm{Fe}$ & $9: 2-7: 6$ & 359.94 & $3.13 \mathrm{E}-02$ & $3.33 \mathrm{E}-01$ & $2.07 E+09$ \\
\hline $3 p 4 f-3 s 5 f$ & $3 \mathrm{Ge}-3 \mathrm{Fo}$ & $7: 5-5: 18$ & 337.56 & $6.20 \mathrm{E}-04$ & $4.82 \mathrm{E}-03$ & $5.08 \mathrm{E}+07$ \\
\hline $3 p 4 f-3 s 5 f$ & $3 \mathrm{Ge}-3 \mathrm{Fo}$ & $7: 5-7: 12$ & 337.56 & $5.05 \mathrm{E}-05$ & $3.93 \mathrm{E}-04$ & $2.96 \mathrm{E}+06$ \\
\hline $3 p 4 f-3 s 5 f$ & $3 \mathrm{Ge}-3 \mathrm{Fo}$ & $7: 5-9: 5$ & 337.33 & $7.09 \mathrm{E}-05$ & $5.52 \mathrm{E}-04$ & $3.23 E+06$ \\
\hline $3 p 4 f-3 s 5 f$ & $3 \mathrm{Ge}-3 \mathrm{Fo}$ & $9: 3-7: 12$ & 345.18 & $6.35 \mathrm{E}-04$ & $6.49 \mathrm{E}-03$ & $4.57 \mathrm{E}+07$ \\
\hline $3 p 4 f-3 s 5 f$ & $3 \mathrm{Ge}-3 \mathrm{Fo}$ & $9: 3-9: 5$ & 344.94 & $1.38 \mathrm{E}-04$ & $1.41 \mathrm{E}-03$ & $7.75 E+06$ \\
\hline $3 p 4 f-3 s 5 f$ & $3 \mathrm{Ge}-3 \mathrm{Fo}$ & $11: 1-9: 5$ & 364.29 & $1.26 \mathrm{E}-03$ & $1.66 \mathrm{E}-02$ & $7.74 \mathrm{E}+07$ \\
\hline$L S$ & $3 \mathrm{Ge}-3 \mathrm{Fo}$ & $27-21$ & & $9.63 \mathrm{E}-04$ & $3.03 \mathrm{E}-02$ & $6.61 E+07$ \\
\hline $3 p 4 f-3 s 5 f$ & $1 \mathrm{Fe}-3 \mathrm{Fo}$ & $7: 6-5: 18$ & 345.18 & $7.75 \mathrm{E}-05$ & $6.16 \mathrm{E}-04$ & $6.07 E+06$ \\
\hline $3 p 4 f-3 s 5 f$ & $1 \mathrm{Fe}-3 \mathrm{Fo}$ & $7: 6-7: 12$ & 345.18 & $2.77 \mathrm{E}-04$ & $2.20 \mathrm{E}-03$ & $1.55 \mathrm{E}+07$ \\
\hline $3 p 4 f-3 s 5 f$ & $1 \mathrm{Fe}-3 \mathrm{Fo}$ & $7: 6-9: 5$ & 344.94 & $6.64 \mathrm{E}-04$ & $5.28 \mathrm{E}-03$ & $2.90 \mathrm{E}+07$ \\
\hline $3 p 3 d-3 p 4 f$ & $1 \mathrm{Fo}-3 \mathrm{Ge}$ & $7: 3-7: 5$ & 75.89 & $2.50 \mathrm{E}-02$ & $4.37 \mathrm{E}-02$ & $2.90 \mathrm{E}+10$ \\
\hline $3 p 3 d-3 p 4 f$ & 1Fo-3Ge & $7: 3-9: 3$ & 75.52 & $1.51 \mathrm{E}-02$ & $2.63 \mathrm{E}-02$ & $1.37 \mathrm{E}+10$ \\
\hline $3 s 4 f-3 p 4 f$ & 1Fo-3Ge & $7: 5-7: 5$ & 389.10 & $4.48 \mathrm{E}-02$ & $4.02 \mathrm{E}-01$ & $1.97 E+09$ \\
\hline $3 s 4 f-3 p 4 f$ & $1 \mathrm{Fo}-3 \mathrm{Ge}$ & $7: 5-9: 3$ & 379.44 & $2.89 \mathrm{E}-03$ & $2.53 \mathrm{E}-02$ & $1.04 \mathrm{E}+08$ \\
\hline $3 p 4 f-3 s 5 f ! 3 p 5 d^{3}$ & $3 \mathrm{Ge}-1 \mathrm{Fo}$ & $7: 5-7: 14$ & 248.42 & $5.08 \mathrm{E}-04$ & $2.91 \mathrm{E}-03$ & $5.49 \mathrm{E}+07$ \\
\hline $3 p 4 f-3 s 5 f ! 3 p 5 d^{3}$ & $3 \mathrm{Ge}-1 \mathrm{Fo}$ & $9: 3-7: 14$ & 252.52 & $2.32 \mathrm{E}-02$ & $1.74 \mathrm{E}-01$ & $3.12 \mathrm{E}+09$ \\
\hline $3 p 4 f-3 s 6 f$ & $3 \mathrm{Ge}-1 \mathrm{Fo}$ & $7: 5-7: 25$ & 140.58 & $4.34 \mathrm{E}-03$ & $1.40 \mathrm{E}-02$ & $1.46 \mathrm{E}+09$ \\
\hline $3 p 4 f-3 s 6 f$ & $3 \mathrm{Ge}-1 \mathrm{Fo}$ & $9: 3-7: 25$ & 141.88 & $3.41 \mathrm{E}-04$ & $1.43 \mathrm{E}-03$ & $1.45 \mathrm{E}+08$ \\
\hline $3 p 3 d-3 p 4 f$ & $1 \mathrm{Fo}-1 \mathrm{Fe}$ & $7: 3-7: 6$ & 75.52 & $2.55 \mathrm{E}-02$ & $4.44 \mathrm{E}-02$ & $2.98 \mathrm{E}+10$ \\
\hline $3 s 4 f-3 p 4 f$ & 1Fo-1Fe & $7: 5-7: 6$ & 379.44 & $4.20 \mathrm{E}-02$ & $3.67 \mathrm{E}-01$ & $1.95 E+09$ \\
\hline $3 p 4 f-3 s 5 f ! 3 p 5 d^{3}$ & 1Fe-1Fo & $7: 6-7: 14$ & 252.52 & $4.72 \mathrm{E}-02$ & $2.75 \mathrm{E}-01$ & $4.94 \mathrm{E}+09$ \\
\hline $3 p 4 f-3 s 6 f$ & 1Fe-1Fo & $7: 6-7: 25$ & 141.88 & $1.94 \mathrm{E}-02$ & $6.34 \mathrm{E}-02$ & $6.43 E+09$ \\
\hline $3 s 3 d-3 s 6 f$ & 3De-3Fo & $7: 1-9: 13$ & 43.39 & $1.40 \mathrm{E}-05$ & $1.40 \mathrm{E}-05$ & $3.87 E+07$ \\
\hline $3 d^{2}-3 s 6 f$ & $3 \mathrm{Fe}-3 \mathrm{Fo}$ & $7: 2-9: 13$ & 61.96 & $1.59 \mathrm{E}-03$ & $2.27 \mathrm{E}-03$ & $2.15 E+09$ \\
\hline $3 d^{2}-3 s 6 f$ & $3 \mathrm{Fe}-3 \mathrm{Fo}$ & $9: 1-9: 13$ & 62.03 & $7.44 \mathrm{E}-05$ & $1.37 \mathrm{E}-04$ & $1.29 \mathrm{E}+08$ \\
\hline$L S$ & $3 \mathrm{Fe}-3 \mathrm{Fo}$ & $21-21$ & & $5.62 \mathrm{E}-04$ & $2.41 \mathrm{E}-03$ & $9.76 \mathrm{E}+08$ \\
\hline $3 s 4 d-3 s 6 f$ & $3 \mathrm{De}-3 \mathrm{Fo}$ & $7: 3-9: 13$ & 104.94 & $5.49 \mathrm{E}-05$ & $1.33 \mathrm{E}-04$ & $2.59 \mathrm{E}+07$ \\
\hline $3 p 4 f-3 s 6 f$ & $3 \mathrm{Ge}-3 \mathrm{Fo}$ & $7: 5-9: 13$ & 165.03 & $4.50 \mathrm{E}-02$ & $1.71 \mathrm{E}-01$ & $8.56 \mathrm{E}+09$ \\
\hline $3 p 4 f-3 s 6 f$ & $3 \mathrm{Ge}-3 \mathrm{Fo}$ & $9: 3-9: 13$ & 166.83 & $4.96 \mathrm{E}-03$ & $2.45 \mathrm{E}-02$ & $1.19 \mathrm{E}+09$ \\
\hline $3 p 4 f-3 s 6 f$ & $3 \mathrm{Ge}-3 \mathrm{Fo}$ & $11: 1-9: 13$ & 171.23 & $1.31 \mathrm{E}-03$ & $8.15 \mathrm{E}-03$ & $3.65 E+08$ \\
\hline$L S$ & $3 \mathrm{Ge}-3 \mathrm{Fo}$ & $27-21$ & & $1.39 \mathrm{E}-02$ & $2.04 \mathrm{E}-01$ & $4.34 \mathrm{E}+09$ \\
\hline $3 p 4 f-3 s 6 f$ & $1 \mathrm{Fe}-3 \mathrm{Fo}$ & $7: 6-9: 13$ & 166.83 & $1.98 \mathrm{E}-02$ & $7.59 \mathrm{E}-02$ & $3.68 \mathrm{E}+09$ \\
\hline $3 p 4 f-3 s 6 f$ & 3De-3Fo & $7: 8-9: 13$ & 174.49 & $2.01 \mathrm{E}-03$ & $8.09 \mathrm{E}-03$ & $3.43 \mathrm{E}+08$ \\
\hline $3 s 5 d-3 s 6 f$ & 3De-3Fo & $7: 10-9: 13$ & 289.18 & $7.72 \mathrm{E}-05$ & $5.15 \mathrm{E}-04$ & $4.79 \mathrm{E}+06$ \\
\hline $3 p 3 d-3 d^{2}$ & $3 F o-1 G e$ & $7: 1-9: 2$ & 213.25 & $1.34 \mathrm{E}-03$ & $6.59 \mathrm{E}-03$ & $1.53 E+08$ \\
\hline $3 p 3 d-3 d^{2}$ & $3 \mathrm{Fo}-1 \mathrm{Ge}$ & $9: 1-9: 2$ & 218.62 & $1.50 \mathrm{E}-04$ & $9.71 \mathrm{E}-04$ & $2.09 \mathrm{E}+07$ \\
\hline $3 p 3 d-3 p 4 f$ & $3 \mathrm{Fo}-1 \mathrm{Ge}$ & $7: 1-9: 5$ & 67.09 & $1.65 \mathrm{E}-02$ & $2.55 \mathrm{E}-02$ & $1.90 \mathrm{E}+10$ \\
\hline $3 p 3 d-3 p 4 f$ & $3 \mathrm{Fo}-1 \mathrm{Ge}$ & $9: 1-9: 5$ & 67.61 & $3.96 \mathrm{E}-05$ & $7.94 \mathrm{E}-05$ & $5.78 E+07$ \\
\hline $3 p 3 d-3 d^{2}$ & 3Do-1Ge & $7: 2-9: 2$ & 242.60 & $9.35 \mathrm{E}-04$ & $5.23 \mathrm{E}-03$ & $8.24 E+07$ \\
\hline $3 p 3 d-3 p 4 f$ & $3 D o-1 G e$ & $7: 2-9: 5$ & 69.74 & $2.28 \mathrm{E}-03$ & $3.67 \mathrm{E}-03$ & $2.43 E+09$ \\
\hline $3 p 3 d-3 d^{2}$ & $1 \mathrm{Fo}-1 \mathrm{Ge}$ & $7: 3-9: 2$ & 290.23 & $3.63 \mathrm{E}-01$ & $2.43 \mathrm{E}+00$ & $2.24 \mathrm{E}+10$ \\
\hline $3 p 3 d-3 p 4 f$ & $1 \mathrm{Fo}-1 \mathrm{Ge}$ & $7: 3-9: 5$ & 73.20 & $7.20 \mathrm{E}-01$ & $1.21 \mathrm{E}+00$ & $6.97 \mathrm{E}+11$ \\
\hline $3 d^{2}-3 s 4 f$ & $1 \mathrm{Ge}-3 \mathrm{Fo}$ & $9: 2-7: 4$ & 142.54 & $5.54 \mathrm{E}-08$ & $2.34 \mathrm{E}-07$ & $2.34 \mathrm{E}+04$ \\
\hline $3 d^{2}-3 s 4 f$ & $1 \mathrm{Ge}-3 \mathrm{Fo}$ & $9: 2-9: 2$ & 142.49 & $4.93 \mathrm{E}-10$ & $2.08 \mathrm{E}-09$ & $1.62 E+02$ \\
\hline $3 s 4 f-3 p 4 f$ & $3 \mathrm{Fo}-1 \mathrm{Ge}$ & $7: 4-9: 5$ & 312.43 & $1.23 \mathrm{E}-03$ & $8.84 \mathrm{E}-03$ & $6.52 \mathrm{E}+07$ \\
\hline $3 s 4 f-3 p 4 f$ & $3 \mathrm{Fo}-1 \mathrm{Ge}$ & $9: 2-9: 5$ & 312.68 & $4.62 \mathrm{E}-03$ & $4.28 \mathrm{E}-02$ & $3.15 E+08$ \\
\hline $3 d^{2}-3 s 4 f$ & $1 \mathrm{Ge}-1 \mathrm{Fo}$ & $9: 2-7: 5$ & 139.65 & $4.79 \mathrm{E}-06$ & $1.98 \mathrm{E}-05$ & $2.11 \mathrm{E}+06$ \\
\hline $3 s 4 f-3 p 4 f$ & 1Fo-1Ge & $7: 5-9: 5$ & 327.29 & $6.20 \mathrm{E}-02$ & $4.67 \mathrm{E}-01$ & $3.00 \mathrm{E}+09$ \\
\hline $3 d^{2}-3 s 5 f$ & $1 \mathrm{Ge}-3 \mathrm{Fo}$ & $9: 2-7: 12$ & 78.78 & $2.48 \mathrm{E}-07$ & $5.79 \mathrm{E}-07$ & $3.43 \mathrm{E}+05$ \\
\hline $3 d^{2}-3 s 5 f$ & $1 G e-3 F o$ & $9: 2-9: 5$ & 78.77 & $1.20 \mathrm{E}-07$ & $2.81 \mathrm{E}-07$ & $1.29 \mathrm{E}+05$ \\
\hline $3 p 4 f-3 s 5 f$ & $1 G e-3 F o$ & $9: 5-7: 12$ & 403.71 & $4.39 \mathrm{E}-05$ & $5.25 \mathrm{E}-04$ & $2.31 \mathrm{E}+06$ \\
\hline $3 p 4 f-3 s 5 f$ & $1 G e-3 F o$ & $9: 5-9: 5$ & 403.38 & $9.15 \mathrm{E}-06$ & $1.09 \mathrm{E}-04$ & $3.75 E+05$ \\
\hline $3 d^{2}-3 s 5 f ! 3 p 5 d^{3}$ & $1 \mathrm{Ge}-1 \mathrm{Fo}$ & $9: 2-7: 14$ & 72.69 & $1.60 \mathrm{E}-05$ & $3.46 \mathrm{E}-05$ & $2.60 \mathrm{E}+07$ \\
\hline
\end{tabular}


Table 2. (continued)

\begin{tabular}{|c|c|c|c|c|c|c|}
\hline$C_{i}-C_{k}$ & $T_{i}-T_{k}$ & $g_{i}: \mathrm{I}-g_{j}: \mathrm{K}$ & $E_{i k}(\AA)$ & $f$ & $S$ & $A\left(\mathrm{~s}^{-1}\right)$ \\
\hline $3 p 4 f-3 s 5 f ! 3 p 5 d^{3}$ & $1 \mathrm{Ge}-1 \mathrm{Fo}$ & $9: 5-7: 14$ & 282.48 & $1.05 \mathrm{E}-03$ & $8.82 \mathrm{E}-03$ & $1.13 \mathrm{E}+08$ \\
\hline $3 d^{2}-3 s 6 f$ & $1 \mathrm{Ge}-1 \mathrm{Fo}$ & $9: 2-7: 25$ & 59.37 & $4.37 \mathrm{E}-05$ & $7.69 \mathrm{E}-05$ & $1.06 \mathrm{E}+08$ \\
\hline $3 p 4 f-3 s 6 f$ & $1 \mathrm{Ge}-1 \mathrm{Fo}$ & $9: 5-7: 25$ & 150.87 & $6.73 \mathrm{E}-05$ & $3.01 \mathrm{E}-04$ & $2.53 \mathrm{E}+07$ \\
\hline $3 d^{2}-3 s 6 f$ & $1 G e-3 F o$ & $9: 2-9: 13$ & 63.33 & $1.53 \mathrm{E}-04$ & $2.87 \mathrm{E}-04$ & $2.55 \mathrm{E}+08$ \\
\hline $3 p 4 f-3 s 6 f$ & $3 \mathrm{Fe}-3 \mathrm{Fo}$ & $9: 4-9: 13$ & 171.23 & $5.75 \mathrm{E}-02$ & $2.92 \mathrm{E}-01$ & $1.31 \mathrm{E}+10$ \\
\hline $3 p 4 f-3 s 6 f$ & $1 \mathrm{Ge}-3 \mathrm{Fo}$ & $9: 5-9: 13$ & 179.40 & $3.71 \mathrm{E}-02$ & $1.97 \mathrm{E}-01$ & $7.68 \mathrm{E}+09$ \\
\hline
\end{tabular}


Table 3

Fine structure energy levels of Fe XV for which forbidden (E2, E3, M1, M2) transitions are presented. See page 583 for Explanation of Tables.

\begin{tabular}{|c|c|c|c|}
\hline$i_{e}$ & $\operatorname{SLp}(\mathrm{cf})$ & $2 J$ & $E$ (Ry) \\
\hline 1 & $1 \mathrm{Se}(1)$ & 0 & $0.00000 \mathrm{E}+00$ \\
\hline 2 & $3 \mathrm{Po}(2)$ & 0 & $2.13090 \mathrm{E}+00$ \\
\hline 3 & $3 \mathrm{Po}(2)$ & 2 & $2.18390 \mathrm{E}+00$ \\
\hline 4 & $3 \mathrm{Po}(2)$ & 4 & $2.31300 \mathrm{E}+00$ \\
\hline 5 & $1 \mathrm{Po}(2)$ & 2 & $3.20680 \mathrm{E}+00$ \\
\hline 6 & $3 \mathrm{Pe}(3)$ & 0 & $5.05320 \mathrm{E}+00$ \\
\hline 7 & 1De(3) & 4 & $5.09950 \mathrm{E}+00$ \\
\hline 8 & $3 \mathrm{Pe}(3)$ & 2 & $5.14500 \mathrm{E}+00$ \\
\hline 9 & $3 \mathrm{Pe}(3)$ & 4 & $5.30180 \mathrm{E}+00$ \\
\hline 10 & $1 \mathrm{Se}(3)$ & 0 & $6.01100 \mathrm{E}+00$ \\
\hline 11 & $3 \mathrm{De}(4)$ & 2 & $6.18540 \mathrm{E}+00$ \\
\hline 12 & $3 \mathrm{De}(4)$ & 4 & $6.19470 \mathrm{E}+00$ \\
\hline 13 & $3 \mathrm{De}(4)$ & 6 & $6.20950 \mathrm{E}+00$ \\
\hline 14 & $1 \mathrm{De}(4)$ & 4 & $6.94470 \mathrm{E}+00$ \\
\hline 15 & $3 \mathrm{Fo}(5)$ & 4 & $8.45880 \mathrm{E}+00$ \\
\hline 16 & $3 \mathrm{Fo}(5)$ & 6 & $8.54880 \mathrm{E}+00$ \\
\hline 17 & $3 \mathrm{Fo}(5)$ & 8 & $8.65390 \mathrm{E}+00$ \\
\hline 18 & 1Do(5) & 4 & $8.64350 \mathrm{E}+00$ \\
\hline 19 & $3 \mathrm{Do}(5)$ & 2 & $8.95660 \mathrm{E}+00$ \\
\hline 20 & $3 \mathrm{Po}(5)$ & 4 & $8.96240 \mathrm{E}+00$ \\
\hline 21 & $3 \mathrm{Po}(5)$ & 0 & $9.07520 \mathrm{E}+00$ \\
\hline 22 & $3 \mathrm{Po}(5)$ & 2 & $9.07840 \mathrm{E}+00$ \\
\hline 23 & $3 \mathrm{Do}(5)$ & 6 & $9.06580 \mathrm{E}+00$ \\
\hline 24 & $3 \mathrm{Do}(5)$ & 4 & $9.08190 \mathrm{E}+00$ \\
\hline 25 & $1 \mathrm{Fo}(5)$ & 6 & $9.68240 \mathrm{E}+00$ \\
\hline 26 & $1 \mathrm{Po}(5)$ & 2 & $9.79510 \mathrm{E}+00$ \\
\hline 27 & $3 \mathrm{Fe}(19)$ & 4 & $1.24870 \mathrm{E}+01$ \\
\hline 28 & $3 \mathrm{Fe}(19)$ & 6 & $1.25030 \mathrm{E}+01$ \\
\hline 29 & $3 F e(19)$ & 8 & $1.25210 \mathrm{E}+01$ \\
\hline 30 & $3 \mathrm{Pe}(19)$ & 4 & $1.28290 \mathrm{E}+01$ \\
\hline 31 & $3 \mathrm{Pe}(19)$ & 0 & $1.29746 \mathrm{E}+01$ \\
\hline 32 & $3 \mathrm{Pe}(19)$ & 2 & $1.29836 \mathrm{E}+01$ \\
\hline 33 & 1De(19) & 4 & $1.27810 \mathrm{E}+01$ \\
\hline 34 & $1 \mathrm{Ge}(19)$ & 8 & $1.28220 \mathrm{E}+01$ \\
\hline 35 & $1 \mathrm{Se}(19)$ & 0 & $1.35510 \mathrm{E}+01$ \\
\hline 36 & $3 \mathrm{Se}(6)$ & 2 & $1.60720 \mathrm{E}+01$ \\
\hline 37 & $1 \mathrm{Se}(6)$ & 0 & $1.62840 \mathrm{E}+01$ \\
\hline 38 & $3 \mathrm{Po}(7)$ & 0 & $1.72016 \mathrm{E}+01$ \\
\hline 39 & $3 \mathrm{Po}(7)$ & 2 & $1.72077 \mathrm{E}+01$ \\
\hline 40 & $3 \mathrm{Po}(7)$ & 4 & $1.72589 \mathrm{E}+01$ \\
\hline 41 & $1 \mathrm{Po}(7)$ & 2 & $1.72230 \mathrm{E}+01$ \\
\hline 42 & $3 \mathrm{De}(8)$ & 2 & $1.85110 \mathrm{E}+01$ \\
\hline 43 & $3 \mathrm{De}(8)$ & 4 & $1.85170 \mathrm{E}+01$ \\
\hline 44 & $3 \mathrm{De}(8)$ & 6 & $1.85280 \mathrm{E}+01$ \\
\hline 45 & $1 \mathrm{De}(8)$ & 4 & $1.85470 \mathrm{E}+01$ \\
\hline 46 & $3 \mathrm{Po}(13)$ & 0 & $1.88070 \mathrm{E}+01$ \\
\hline 47 & $3 \mathrm{Po}(13)$ & 2 & $1.88400 \mathrm{E}+01$ \\
\hline 48 & $3 \mathrm{Po}(13)$ & 4 & $1.89794 \mathrm{E}+01$ \\
\hline 49 & $1 \mathrm{Po}(13)$ & 2 & $1.91124 \mathrm{E}+01$ \\
\hline 50 & $3 \mathrm{Fo}(9)$ & 4 & $1.92140 \mathrm{E}+01$ \\
\hline 51 & $3 \mathrm{Fo}(9)$ & 6 & $1.92150 \mathrm{E}+01$ \\
\hline 52 & $3 \mathrm{Fo}(9)$ & 8 & $1.92180 \mathrm{E}+01$ \\
\hline 53 & $1 \mathrm{Fo}(9)$ & 6 & $1.93480 \mathrm{E}+01$ \\
\hline 54 & $1 \mathrm{Pe}(14)$ & 2 & $1.97201 \mathrm{E}+01$ \\
\hline 55 & $3 \mathrm{De}(14)$ & 2 & $1.98396 \mathrm{E}+01$ \\
\hline 56 & $3 \mathrm{De}(14)$ & 4 & $1.98639 \mathrm{E}+01$ \\
\hline 57 & $3 \mathrm{Pe}(14)$ & 0 & $1.98988 \mathrm{E}+01$ \\
\hline 58 & $3 \mathrm{Pe}(14)$ & 2 & $1.99625 \mathrm{E}+01$ \\
\hline 59 & $3 \mathrm{De}(14)$ & 6 & $1.99898 \mathrm{E}+01$ \\
\hline 60 & $3 \mathrm{Pe}(14)$ & 4 & $2.00312 \mathrm{E}+01$ \\
\hline 61 & $3 \mathrm{Se}(14)$ & 2 & $2.00710 \mathrm{E}+01$ \\
\hline 62 & $1 \mathrm{De}(14)$ & 4 & $2.02511 \mathrm{E}+01$ \\
\hline 63 & $1 \mathrm{Se}(14)$ & 0 & $2.05245 \mathrm{E}+01$ \\
\hline 64 & $1 \mathrm{Do}(15)$ & 4 & $2.11549 \mathrm{E}+01$ \\
\hline 65 & $3 \mathrm{Do}(15)$ & 2 & $2.11744 \mathrm{E}+01$ \\
\hline 66 & $3 \mathrm{Do}(15)$ & 4 & $2.11774 \mathrm{E}+01$ \\
\hline 67 & 3Do(15) & 6 & $2.12018 \mathrm{E}+01$ \\
\hline 68 & $3 \mathrm{Fo}(15)$ & 4 & $2.13085 E+01$ \\
\hline 69 & $3 F o(15)$ & 6 & $2.13334 \mathrm{E}+01$ \\
\hline 70 & $3 \mathrm{Fo}(15)$ & 8 & $2.13847 \mathrm{E}+01$ \\
\hline 71 & $3 \mathrm{Po}(15)$ & 4 & $2.14277 \mathrm{E}+01$ \\
\hline 72 & $3 \mathrm{Po}(15)$ & 2 & $2.14441 \mathrm{E}+01$ \\
\hline 73 & $1 \mathrm{Fo}(15)$ & 6 & $2.14621 \mathrm{E}+01$ \\
\hline 74 & $3 \mathrm{Po}(15)$ & 0 & $2.14681 \mathrm{E}+01$ \\
\hline 75 & $1 \mathrm{Po}(15)$ & 2 & $2.15607 \mathrm{E}+01$ \\
\hline
\end{tabular}

Table 3. (continued)

\begin{tabular}{|c|c|c|c|}
\hline$i_{e}$ & $\operatorname{SLp}(\mathrm{cf})$ & $2 J$ & $E$ (Ry) \\
\hline 76 & $3 \mathrm{Ge}(16)$ & 6 & $2.16900 \mathrm{E}+01$ \\
\hline 77 & $1 \mathrm{Fe}(16)$ & 6 & $2.17490 \mathrm{E}+01$ \\
\hline 78 & $3 \mathrm{Ge}(16)$ & 8 & $2.17490 \mathrm{E}+01$ \\
\hline 79 & $3 \mathrm{Fe}(16)$ & 4 & $2.17800 \mathrm{E}+01$ \\
\hline 80 & $3 \mathrm{Fe}(16)$ & 6 & $2.19173 E+01$ \\
\hline 81 & $3 \mathrm{Fe}(16)$ & 8 & $2.18900 \mathrm{E}+01$ \\
\hline 82 & $3 \mathrm{Ge}(16)$ & 10 & $2.18900 \mathrm{E}+01$ \\
\hline 83 & $3 \operatorname{De}(16)$ & 6 & $2.19890 \mathrm{E}+01$ \\
\hline 84 & $3 \operatorname{De}(16)$ & 4 & $2.20010 \mathrm{E}+01$ \\
\hline 85 & $3 \operatorname{De}(16)$ & 2 & $2.20540 \mathrm{E}+01$ \\
\hline 86 & $1 \mathrm{Ge}(16)$ & 8 & $2.21320 \mathrm{E}+01$ \\
\hline 87 & $1 \mathrm{De}(16)$ & 4 & $2.21980 \mathrm{E}+01$ \\
\hline 88 & $3 \mathrm{Se}(10)$ & 2 & $2.31900 \mathrm{E}+01$ \\
\hline 89 & $1 \mathrm{Se}(10)$ & 0 & $2.30314 \mathrm{E}+01$ \\
\hline 90 & $3 \mathrm{Po}(11)$ & 0 & $2.34461 \mathrm{E}+01$ \\
\hline 91 & $3 \mathrm{Po}(11)$ & 2 & $2.34522 \mathrm{E}+01$ \\
\hline 92 & $3 \mathrm{Po}(11)$ & 4 & $2.34727 \mathrm{E}+01$ \\
\hline 93 & $1 \mathrm{Po}(11)$ & 2 & $2.33920 \mathrm{E}+01$ \\
\hline 94 & $3 \mathrm{De}(12)$ & 2 & $2.40580 \mathrm{E}+01$ \\
\hline 95 & $3 \mathrm{De}(12)$ & 4 & $2.40570 \mathrm{E}+01$ \\
\hline 96 & $3 \operatorname{De}(12)$ & 6 & $2.40600 \mathrm{E}+01$ \\
\hline 97 & $1 \mathrm{De}(12)$ & 4 & $2.41384 \mathrm{E}+01$ \\
\hline 98 & $3 F o(20)$ & 4 & $2.43890 \mathrm{E}+01$ \\
\hline 99 & $3 F o(20)$ & 6 & $2.43890 \mathrm{E}+01$ \\
\hline 100 & $3 \mathrm{Fo}(20)$ & 8 & $2.43910 \mathrm{E}+01$ \\
\hline 101 & $1 \mathrm{Fo}(20)$ & 6 & $2.53580 \mathrm{E}+01$ \\
\hline 102 & $3 \mathrm{Se}(17)$ & 2 & $5.38742 \mathrm{E}+01$ \\
\hline 103 & $3 \operatorname{De}(17)$ & 4 & $5.40548 \mathrm{E}+01$ \\
\hline 104 & $3 \operatorname{De}(17)$ & 6 & $5.41476 \mathrm{E}+01$ \\
\hline 105 & $3 \mathrm{Pe}(17)$ & 2 & $5.42275 \mathrm{E}+01$ \\
\hline 106 & $3 \mathrm{Pe}(17)$ & 4 & $5.43337 \mathrm{E}+01$ \\
\hline 107 & $3 \mathrm{Pe}(17)$ & 0 & $5.47663 \mathrm{E}+01$ \\
\hline 108 & $1 \mathrm{Pe}(17)$ & 2 & $5.49536 \mathrm{E}+01$ \\
\hline 109 & $3 \operatorname{De}(17)$ & 2 & $5.51546 \mathrm{E}+01$ \\
\hline 110 & 1De(17) & 4 & $5.51773 \mathrm{E}+01$ \\
\hline 111 & $1 \mathrm{Se}(17)$ & 0 & $5.61908 \mathrm{E}+01$ \\
\hline 112 & $3 \mathrm{Po}(18)$ & 0 & $5.78646 \mathrm{E}+01$ \\
\hline 113 & $3 \mathrm{Po}(18)$ & 2 & $5.79289 \mathrm{E}+01$ \\
\hline 114 & $3 F o(18)$ & 8 & $5.80344 \mathrm{E}+01$ \\
\hline 115 & $3 \mathrm{Po}(18)$ & 4 & $5.80450 \mathrm{E}+01$ \\
\hline 116 & $3 F o(18)$ & 6 & $5.80719 \mathrm{E}+01$ \\
\hline 117 & $3 \mathrm{Do}(18)$ & 4 & $5.81797 \mathrm{E}+01$ \\
\hline 118 & 3Do(18) & 6 & $5.82470 \mathrm{E}+01$ \\
\hline 119 & $3 \mathrm{Do}(18)$ & 2 & $5.85566 \mathrm{E}+01$ \\
\hline 120 & $3 \mathrm{Fo}(18)$ & 4 & $5.89991 \mathrm{E}+01$ \\
\hline 121 & $1 \mathrm{Do}(18)$ & 4 & $5.90576 \mathrm{E}+01$ \\
\hline 122 & $1 \mathrm{Fo}(18)$ & 6 & $5.90879 \mathrm{E}+01$ \\
\hline 123 & $1 \mathrm{Po}(18)$ & 2 & $5.95062 \mathrm{E}+01$ \\
\hline
\end{tabular}


Table 4

Partial set of radiative decay rates for forbidden E2, M1, E3, M2 transitions in Fe XV. See page 583 for Explanation of Tables.

\begin{tabular}{|c|c|c|c|c|c|c|c|}
\hline$\underline{i-j}$ & $T_{i} C_{i}-T_{j} C_{j}$ & $g_{i}-g_{j}$ & $\lambda(\AA)$ & $E_{i}(\mathrm{Ry})$ & $E_{j}(\mathrm{Ry})$ & AE2 & AM1 \\
\hline \multicolumn{8}{|c|}{$E 2$ and $M 1, N_{t r}=5066$} \\
\hline $2-3$ & 3Рo 2-3Рo 2 & $1-3$ & 17193 & $2.13 \mathrm{E}+00$ & $2.18 \mathrm{E}+00$ & $0.00 \mathrm{E}+00$ & $3.52 \mathrm{E}+00$ \\
\hline $2-4$ & 3Po 2-3Po 2 & $1-5$ & 5004 & $2.13 \mathrm{E}+00$ & $2.31 \mathrm{E}+00$ & $9.18 \mathrm{E}-03$ & $0.00 \mathrm{E}+00$ \\
\hline $3-4$ & 3Рo 2-3Рo 2 & $3-5$ & 7058 & $2.18 \mathrm{E}+00$ & $2.31 \mathrm{E}+00$ & $3.69 \mathrm{E}-03$ & $3.81 \mathrm{E}+01$ \\
\hline $2-5$ & 3Рo 2-1Po 2 & $1-3$ & 846.98 & $2.13 \mathrm{E}+00$ & $3.21 \mathrm{E}+00$ & $0.00 \mathrm{E}+00$ & $1.70 \mathrm{E}+02$ \\
\hline $3-5$ & 3Рo 2-1Po 2 & $3-3$ & 890.87 & $2.18 \mathrm{E}+00$ & $3.21 \mathrm{E}+00$ & $2.19 \mathrm{E}+00$ & $1.12 \mathrm{E}+02$ \\
\hline $4-5$ & 3Po 2-1Po 2 & $5-3$ & 1019 & $2.31 \mathrm{E}+00$ & $3.21 \mathrm{E}+00$ & $6.04 \mathrm{E}-01$ & $1.24 \mathrm{E}+02$ \\
\hline $1-7$ & 1Se 1-1De 3 & $1-5$ & 178.70 & $0.00 \mathrm{E}+00$ & $5.10 \mathrm{E}+00$ & $1.59 \mathrm{E}+05$ & $0.00 \mathrm{E}+00$ \\
\hline $6-7$ & 3Pe 3-1De 3 & $1-5$ & 19681 & $5.05 \mathrm{E}+00$ & $5.10 \mathrm{E}+00$ & $3.34 \mathrm{E}-06$ & $0.00 \mathrm{E}+00$ \\
\hline $1-8$ & 1Se $1-3 \mathrm{Pe} 3$ & $1-3$ & 177.12 & $0.00 \mathrm{E}+00$ & $5.14 \mathrm{E}+00$ & $0.00 \mathrm{E}+00$ & $8.42 \mathrm{E}+01$ \\
\hline $6-8$ & 3Pe 3-3Pe 3 & $1-3$ & 9926 & $5.05 \mathrm{E}+00$ & $5.14 \mathrm{E}+00$ & $0.00 \mathrm{E}+00$ & $1.79 \mathrm{E}+01$ \\
\hline $7-8$ & 1De 3-3Pe 3 & $5-3$ & 20027 & $5.10 \mathrm{E}+00$ & $5.14 \mathrm{E}+00$ & $4.35 \mathrm{E}-06$ & $4.13 \mathrm{E}-01$ \\
\hline $1-9$ & 1Se $1-3 \mathrm{Pe} 3$ & $1-5$ & 171.88 & $0.00 \mathrm{E}+00$ & $5.30 \mathrm{E}+00$ & $3.81 \mathrm{E}+04$ & $0.00 \mathrm{E}+00$ \\
\hline $6-9$ & 3 Pe $3-3$ Pe 3 & $1-5$ & 3665 & $5.05 E+00$ & $5.30 \mathrm{E}+00$ & $2.51 \mathrm{E}-02$ & $0.00 \mathrm{E}+00$ \\
\hline $7-9$ & 1De 3-3Pe 3 & $5-5$ & 4504 & $5.10 \mathrm{E}+00$ & $5.30 \mathrm{E}+00$ & $2.45 \mathrm{E}-02$ & $5.55 \mathrm{E}+01$ \\
\hline $8-9$ & 3Рe 3-3Pe 3 & $3-5$ & 5811 & $5.14 \mathrm{E}+00$ & $5.30 \mathrm{E}+00$ & $7.26 \mathrm{E}-03$ & $5.86 \mathrm{E}+01$ \\
\hline $7-10$ & 1De 3-1Se 3 & $5-1$ & 999 & $5.10 \mathrm{E}+00$ & $6.01 \mathrm{E}+00$ & $2.70 \mathrm{E}+02$ & $0.00 \mathrm{E}+00$ \\
\hline $8-10$ & $3 \mathrm{Pe} 3-1 \mathrm{Se} 3$ & $3-1$ & 1052 & $5.14 \mathrm{E}+00$ & $6.01 \mathrm{E}+00$ & $0.00 \mathrm{E}+00$ & $1.25 E+03$ \\
\hline $9-10$ & 3Pe 3-1Se 3 & $5-1$ & 1284 & $5.30 \mathrm{E}+00$ & $6.01 \mathrm{E}+00$ & $2.13 \mathrm{E}+01$ & $0.00 \mathrm{E}+00$ \\
\hline $1-11$ & 1Se 1-3De 4 & $1-3$ & 147.33 & $0.00 \mathrm{E}+00$ & $6.19 E+00$ & $0.00 \mathrm{E}+00$ & $1.39 \mathrm{E}+00$ \\
\hline $6-11$ & 3Pe 3-3De 4 & $1-3$ & 804.86 & $5.05 E+00$ & $6.19 E+00$ & $0.00 \mathrm{E}+00$ & $2.26 \mathrm{E}-04$ \\
\hline $7-11$ & 1De 3-3De 4 & $5-3$ & 839.18 & $5.10 \mathrm{E}+00$ & $6.19 E+00$ & $8.04 \mathrm{E}-02$ & $3.23 E+00$ \\
\hline $8-11$ & 3Pe 3-3De 4 & $3-3$ & 875.88 & $5.14 \mathrm{E}+00$ & $6.19 \mathrm{E}+00$ & $8.17 \mathrm{E}-01$ & $1.29 \mathrm{E}-04$ \\
\hline $9-11$ & 3Pe 3-3De 4 & $5-3$ & 1031 & $5.30 \mathrm{E}+00$ & $6.19 \mathrm{E}+00$ & $9.29 \mathrm{E}-02$ & $5.47 \mathrm{E}-01$ \\
\hline $10-11$ & 1Se 3-3De 4 & $1-3$ & 5225 & $6.01 \mathrm{E}+00$ & $6.19 \mathrm{E}+00$ & $0.00 \mathrm{E}+00$ & $9.56 \mathrm{E}-07$ \\
\hline $1-12$ & 1Se 1-3De 4 & $1-5$ & 147.10 & $0.00 \mathrm{E}+00$ & $6.19 E+00$ & $1.76 \mathrm{E}+02$ & $0.00 \mathrm{E}+00$ \\
\hline $6-12$ & 3Pe 3-3De 4 & $1-5$ & 798.31 & $5.05 \mathrm{E}+00$ & $6.19 \mathrm{E}+00$ & $5.08 \mathrm{E}-01$ & $0.00 \mathrm{E}+00$ \\
\hline $7-12$ & 1De 3-3De 4 & $5-5$ & 832.06 & $5.10 \mathrm{E}+00$ & $6.19 E+00$ & $3.22 \mathrm{E}-02$ & $4.83 \mathrm{E}-01$ \\
\hline $8-12$ & 3Pe 3-3De 4 & $3-5$ & 868.12 & $5.14 \mathrm{E}+00$ & $6.19 \mathrm{E}+00$ & $1.02 \mathrm{E}-01$ & $3.50 \mathrm{E}-02$ \\
\hline $9-12$ & 3Pe 3-3De 4 & $5-5$ & 1020 & $5.30 \mathrm{E}+00$ & $6.19 E+00$ & $2.88 \mathrm{E}-01$ & $2.40 \mathrm{E}-06$ \\
\hline $10-12$ & 1Se 3-3De 4 & $1-5$ & 4960 & $6.01 \mathrm{E}+00$ & $6.19 \mathrm{E}+00$ & $1.83 \mathrm{E}-05$ & $0.00 \mathrm{E}+00$ \\
\hline $11-12$ & 3De 4-3De 4 & $3-5$ & 97988 & $6.19 \mathrm{E}+00$ & $6.19 \mathrm{E}+00$ & $2.61 \mathrm{E}-09$ & $2.58 \mathrm{E}-02$ \\
\hline $7-13$ & 1De 3-3De 4 & $5-7$ & 820.96 & $5.10 \mathrm{E}+00$ & $6.21 \mathrm{E}+00$ & $1.15 \mathrm{E}-01$ & $1.50 \mathrm{E}+00$ \\
\hline $8-13$ & 3Pe 3-3De 4 & $3-7$ & 856.05 & $5.14 \mathrm{E}+00$ & $6.21 \mathrm{E}+00$ & $4.18 \mathrm{E}-01$ & $0.00 \mathrm{E}+00$ \\
\hline $9-13$ & 3Pe 3-3De 4 & $5-7$ & 1003 & $5.30 \mathrm{E}+00$ & $6.21 \mathrm{E}+00$ & $3.43 \mathrm{E}-01$ & $2.51 \mathrm{E}-01$ \\
\hline $11-13$ & 3De 4-3De 4 & $3-7$ & 37812 & $6.19 E+00$ & $6.21 \mathrm{E}+00$ & $2.49 \mathrm{E}-08$ & $0.00 \mathrm{E}+00$ \\
\hline $12-13$ & 3De 4-3De 4 & $5-7$ & 61571 & $6.19 \mathrm{E}+00$ & $6.21 \mathrm{E}+00$ & $2.18 \mathrm{E}-08$ & $7.70 \mathrm{E}-02$ \\
\hline $1-14$ & 1Se 1-1De 4 & $1-5$ & 131.22 & $0.00 \mathrm{E}+00$ & $6.94 \mathrm{E}+00$ & $1.73 E+06$ & $0.00 \mathrm{E}+00$ \\
\hline $6-14$ & 3Pe 3-1De 4 & $1-5$ & 481.77 & $5.05 \mathrm{E}+00$ & $6.94 \mathrm{E}+00$ & $2.29 \mathrm{E}+01$ & $0.00 \mathrm{E}+00$ \\
\hline $7-14$ & 1De 3-1De 4 & $5-5$ & 493.86 & $5.10 \mathrm{E}+00$ & $6.94 \mathrm{E}+00$ & $1.37 \mathrm{E}+02$ & $2.08 \mathrm{E}+01$ \\
\hline $8-14$ & 3Pe 3-1De 4 & $3-5$ & 506.34 & $5.14 \mathrm{E}+00$ & $6.94 \mathrm{E}+00$ & $5.73 \mathrm{E}-01$ & $4.16 \mathrm{E}+01$ \\
\hline $9-14$ & 3Pe 3-1De 4 & $5-5$ & 554.67 & $5.30 \mathrm{E}+00$ & $6.94 \mathrm{E}+00$ & $4.77 \mathrm{E}+00$ & $8.46 \mathrm{E}+01$ \\
\hline $10-14$ & 1Se 3-1De 4 & $1-5$ & 975.97 & $6.01 \mathrm{E}+00$ & $6.94 \mathrm{E}+00$ & $2.70 \mathrm{E}+01$ & $0.00 \mathrm{E}+00$ \\
\hline $11-14$ & 3De 4-1De 4 & $3-5$ & 1200 & $6.19 E+00$ & $6.94 \mathrm{E}+00$ & $3.21 \mathrm{E}-03$ & $5.37 \mathrm{E}+00$ \\
\hline $12-14$ & 3De 4-1De 4 & $5-5$ & 1215 & $6.19 \mathrm{E}+00$ & $6.94 \mathrm{E}+00$ & $2.45 \mathrm{E}-03$ & $1.07 \mathrm{E}+00$ \\
\hline $13-14$ & 3De 4-1De 4 & $7-5$ & 1239 & $6.21 \mathrm{E}+00$ & $6.94 \mathrm{E}+00$ & $5.07 \mathrm{E}-03$ & $5.18 \mathrm{E}+00$ \\
\hline $2-15$ & 3Po 2-3Fo 5 & $1-5$ & 144.01 & $2.13 \mathrm{E}+00$ & $8.46 \mathrm{E}+00$ & $4.41 \mathrm{E}+05$ & $0.00 \mathrm{E}+00$ \\
\hline $3-15$ & 3Рo 2-3Fo 5 & $3-5$ & 145.22 & $2.18 \mathrm{E}+00$ & $8.46 \mathrm{E}+00$ & $4.39 \mathrm{E}+05$ & $1.85 \mathrm{E}+00$ \\
\hline $4-15$ & 3Рo 2-3Fo 5 & $5-5$ & 148.27 & $2.31 \mathrm{E}+00$ & $8.46 \mathrm{E}+00$ & $3.32 \mathrm{E}+04$ & $6.80 \mathrm{E}-01$ \\
\hline $5-15$ & 1Po 2-3Fo 5 & $3-5$ & 173.51 & $3.21 \mathrm{E}+00$ & $8.46 \mathrm{E}+00$ & $3.63 E+04$ & $6.05 \mathrm{E}-01$ \\
\hline $3-16$ & 3Рo 2-3Fo 5 & $3-7$ & 143.17 & $2.18 \mathrm{E}+00$ & $8.55 \mathrm{E}+00$ & $7.65 \mathrm{E}+05$ & $0.00 \mathrm{E}+00$ \\
\hline $4-16$ & 3Рo 2-3Fo 5 & $5-7$ & 146.13 & $2.31 \mathrm{E}+00$ & $8.55 E+00$ & $2.51 \mathrm{E}+05$ & $8.20 \mathrm{E}-01$ \\
\hline $5-16$ & 1Po 2-3Fo 5 & $3-7$ & 170.59 & $3.21 \mathrm{E}+00$ & $8.55 \mathrm{E}+00$ & $5.62 \mathrm{E}+02$ & $0.00 \mathrm{E}+00$ \\
\hline $15-16$ & 3Fo 5-3Fo 5 & $5-7$ & 10125 & $8.46 \mathrm{E}+00$ & $8.55 \mathrm{E}+00$ & $2.86 \mathrm{E}-04$ & $2.20 \mathrm{E}+01$ \\
\hline $4-17$ & 3Po 2-3Fo 5 & $5-9$ & 143.71 & $2.31 \mathrm{E}+00$ & $8.65 \mathrm{E}+00$ & $1.03 \mathrm{E}+06$ & $0.00 \mathrm{E}+00$ \\
\hline $15-17$ & 3Fo 5-3Fo 5 & $5-9$ & 4670 & $8.46 \mathrm{E}+00$ & $8.65 E+00$ & $3.45 \mathrm{E}-04$ & $0.00 \mathrm{E}+00$ \\
\hline $16-17$ & 3Fo 5-3Fo 5 & $7-9$ & 8670 & $8.55 \mathrm{E}+00$ & $8.65 E+00$ & $5.80 \mathrm{E}-04$ & $3.07 \mathrm{E}+01$ \\
\hline $2-18$ & 3Po 2-1Do 5 & $1-5$ & 139.92 & $2.13 \mathrm{E}+00$ & $8.64 \mathrm{E}+00$ & $1.17 \mathrm{E}+05$ & $0.00 \mathrm{E}+00$ \\
\hline $3-18$ & 3Po 2-1Do 5 & $3-5$ & 141.07 & $2.18 \mathrm{E}+00$ & $8.64 \mathrm{E}+00$ & $1.79 \mathrm{E}+03$ & $3.57 \mathrm{E}+00$ \\
\hline $4-18$ & 3Po 2-1Do 5 & $5-5$ & 143.95 & $2.31 \mathrm{E}+00$ & $8.64 \mathrm{E}+00$ & $1.36 \mathrm{E}+04$ & $2.81 \mathrm{E}+00$ \\
\hline $5-18$ & 1Po 2-1Do 5 & $3-5$ & 167.61 & $3.21 \mathrm{E}+00$ & $8.64 \mathrm{E}+00$ & $4.80 \mathrm{E}+05$ & $7.88 \mathrm{E}-01$ \\
\hline $15-18$ & 3Fo 5-1Do 5 & $5-5$ & 4933 & $8.46 \mathrm{E}+00$ & $8.64 \mathrm{E}+00$ & $5.59 \mathrm{E}-03$ & $1.44 \mathrm{E}+01$ \\
\hline $16-18$ & 3Fo 5-1Do 5 & $7-5$ & 9622 & $8.55 \mathrm{E}+00$ & $8.64 \mathrm{E}+00$ & $2.80 \mathrm{E}-05$ & $4.35 \mathrm{E}+00$ \\
\hline $17-18$ & 3Fo 5-1Do 5 & $9-5$ & 87623 & $8.65 E+00$ & $8.64 \mathrm{E}+00$ & $9.11 \mathrm{E}-10$ & $0.00 \mathrm{E}+00$ \\
\hline $2-19$ & 3Po 2-3Do 5 & $1-3$ & 133.51 & $2.13 \mathrm{E}+00$ & $8.96 \mathrm{E}+00$ & $0.00 \mathrm{E}+00$ & $2.67 \mathrm{E}-01$ \\
\hline $3-19$ & 3Po 2-3Do 5 & $3-3$ & 134.55 & $2.18 \mathrm{E}+00$ & $8.96 \mathrm{E}+00$ & $1.03 \mathrm{E}+06$ & $2.72 \mathrm{E}+00$ \\
\hline $4-19$ & 3Рo 2-3Do 5 & $5-3$ & 137.16 & $2.31 \mathrm{E}+00$ & $8.96 \mathrm{E}+00$ & $7.04 \mathrm{E}+02$ & $1.04 \mathrm{E}+01$ \\
\hline $5-19$ & 1Po 2-3Do 5 & $3-3$ & 158.49 & $3.21 \mathrm{E}+00$ & $8.96 \mathrm{E}+00$ & $9.06 \mathrm{E}+02$ & $9.18 \mathrm{E}+00$ \\
\hline $15-19$ & 3Fo 5-3Do 5 & $5-3$ & 1830 & $8.46 \mathrm{E}+00$ & $8.96 \mathrm{E}+00$ & $1.61 \mathrm{E}-01$ & $8.17 \mathrm{E}+00$ \\
\hline $16-19$ & 3Fo 5-3Do 5 & $7-3$ & 2234 & $8.55 \mathrm{E}+00$ & $8.96 \mathrm{E}+00$ & $6.43 \mathrm{E}-01$ & $0.00 \mathrm{E}+00$ \\
\hline $18-19$ & 1Do 5-3Do 5 & $5-3$ & 2910 & $8.64 \mathrm{E}+00$ & $8.96 \mathrm{E}+00$ & $5.82 \mathrm{E}-03$ & $2.38 \mathrm{E}+01$ \\
\hline $2-20$ & 3Рo 2-3Рo 5 & $1-5$ & 133.39 & $2.13 \mathrm{E}+00$ & $8.96 \mathrm{E}+00$ & $4.62 \mathrm{E}+05$ & $0.00 \mathrm{E}+00$ \\
\hline $3-20$ & 3Рo 2-3Рo 5 & $3-5$ & 134.43 & $2.18 \mathrm{E}+00$ & $8.96 \mathrm{E}+00$ & $5.41 \mathrm{E}+05$ & $2.05 \mathrm{E}+00$ \\
\hline $4-20$ & 3Рo 2-3Рo 5 & $5-5$ & 137.04 & $2.31 \mathrm{E}+00$ & $8.96 \mathrm{E}+00$ & $6.38 \mathrm{E}+03$ & $6.75 \mathrm{E}-01$ \\
\hline $5-20$ & 1Po 2-3Рo 5 & $3-5$ & 158.33 & $3.21 \mathrm{E}+00$ & $8.96 \mathrm{E}+00$ & $2.72 \mathrm{E}+04$ & $2.28 \mathrm{E}+01$ \\
\hline
\end{tabular}


Table 4. (continued)

\begin{tabular}{|c|c|c|c|c|c|c|c|}
\hline$i-j$ & $T_{i} C_{i}-T_{j} C_{j}$ & $g_{i}-g_{j}$ & $\lambda(\AA)$ & $E_{i}$ (Ry) & $E_{j}(\mathrm{Ry})$ & AE2 & AM1 \\
\hline $15-20$ & 3Fo 5-3Рo 5 & $5-5$ & 1809 & $8.46 \mathrm{E}+00$ & $8.96 \mathrm{E}+00$ & $9.43 \mathrm{E}-03$ & $7.41 \mathrm{E}+00$ \\
\hline $16-20$ & 3Fo 5-3Рo 5 & $7-5$ & 2203 & $8.55 \mathrm{E}+00$ & $8.96 \mathrm{E}+00$ & $2.27 \mathrm{E}-01$ & $1.17 \mathrm{E}+00$ \\
\hline $17-20$ & 3Fo 5-3Рo 5 & $9-5$ & 2953 & $8.65 \mathrm{E}+00$ & $8.96 \mathrm{E}+00$ & $3.13 \mathrm{E}-01$ & $0.00 \mathrm{E}+00$ \\
\hline $18-20$ & 1Do 5-3Po 5 & $5-5$ & 2857 & $8.64 \mathrm{E}+00$ & $8.96 \mathrm{E}+00$ & $1.24 \mathrm{E}-02$ & $3.64 \mathrm{E}+01$ \\
\hline $19-20$ & 3Do 5-3Po 5 & $3-5$ & 157108 & $8.96 \mathrm{E}+00$ & $8.96 \mathrm{E}+00$ & $2.95 \mathrm{E}-11$ & $4.44 \mathrm{E}-03$ \\
\hline $3-21$ & 3Рo 2-3Рo 5 & $3-1$ & 132.23 & $2.18 \mathrm{E}+00$ & $9.08 \mathrm{E}+00$ & $0.00 \mathrm{E}+00$ & $1.29 \mathrm{E}+01$ \\
\hline $4-21$ & 3Рo 2-3Рo 5 & $5-1$ & 134.76 & $2.31 \mathrm{E}+00$ & $9.08 \mathrm{E}+00$ & $1.04 \mathrm{E}+06$ & $0.00 \mathrm{E}+00$ \\
\hline $5-21$ & 1Po 2-3Рo 5 & $3-1$ & 155.28 & $3.21 \mathrm{E}+00$ & $9.08 \mathrm{E}+00$ & $0.00 \mathrm{E}+00$ & $6.02 \mathrm{E}+00$ \\
\hline $15-21$ & 3Fo 5-3Po 5 & $5-1$ & 1478 & $8.46 \mathrm{E}+00$ & $9.08 \mathrm{E}+00$ & $1.49 \mathrm{E}+01$ & $0.00 \mathrm{E}+00$ \\
\hline $18-21$ & 1Do 5-3Po 5 & $5-1$ & 2110 & $8.64 \mathrm{E}+00$ & $9.08 \mathrm{E}+00$ & $3.41 \mathrm{E}-01$ & $0.00 \mathrm{E}+00$ \\
\hline $19-21$ & 3Do 5-3Po 5 & $3-1$ & 7683 & $8.96 \mathrm{E}+00$ & $9.08 \mathrm{E}+00$ & $0.00 \mathrm{E}+00$ & $3.15 E+01$ \\
\hline $20-21$ & 3Рo 5-3Ро 5 & $5-1$ & 8078 & $8.96 \mathrm{E}+00$ & $9.08 \mathrm{E}+00$ & $5.61 \mathrm{E}-04$ & $0.00 \mathrm{E}+00$ \\
\hline $2-22$ & 3Рo 2-3Рo 5 & $1-3$ & 131.16 & $2.13 \mathrm{E}+00$ & $9.08 \mathrm{E}+00$ & $0.00 \mathrm{E}+00$ & $7.81 \mathrm{E}-01$ \\
\hline $3-22$ & 3Рo 2-3Рo 5 & $3-3$ & 132.17 & $2.18 \mathrm{E}+00$ & $9.08 \mathrm{E}+00$ & $2.36 \mathrm{E}+02$ & $6.47 \mathrm{E}-01$ \\
\hline $4-22$ & 3Рo 2-3Рo 5 & $5-3$ & 134.70 & $2.31 \mathrm{E}+00$ & $9.08 \mathrm{E}+00$ & $1.03 \mathrm{E}+06$ & $5.56 \mathrm{E}+00$ \\
\hline $5-22$ & 1Po 2-3Рo 5 & $3-3$ & 155.20 & $3.21 \mathrm{E}+00$ & $9.08 \mathrm{E}+00$ & $3.99 \mathrm{E}+01$ & $1.81 \mathrm{E}+01$ \\
\hline $15-22$ & 3Fo 5-3Рo 5 & $5-3$ & 1470 & $8.46 \mathrm{E}+00$ & $9.08 \mathrm{E}+00$ & $6.86 \mathrm{E}+00$ & $2.73 \mathrm{E}+01$ \\
\hline $16-22$ & 3Fo 5-3Рo 5 & $7-3$ & 1720 & $8.55 \mathrm{E}+00$ & $9.08 \mathrm{E}+00$ & $2.90 \mathrm{E}+00$ & $0.00 \mathrm{E}+00$ \\
\hline $18-22$ & 1Do 5-3Po 5 & $5-3$ & 2095 & $8.64 \mathrm{E}+00$ & $9.08 \mathrm{E}+00$ & $1.11 \mathrm{E}-01$ & $1.71 \mathrm{E}+01$ \\
\hline $19-22$ & 3Do 5-3Рo 5 & $3-3$ & 7481 & $8.96 \mathrm{E}+00$ & $9.08 \mathrm{E}+00$ & $3.83 \mathrm{E}-04$ & $2.50 \mathrm{E}+01$ \\
\hline $20-22$ & 3Рo 5-3Рo 5 & $5-3$ & 7855 & $8.96 \mathrm{E}+00$ & $9.08 \mathrm{E}+00$ & $6.51 \mathrm{E}-06$ & $1.33 \mathrm{E}+00$ \\
\hline $21-22$ & 3Рo 5-3Ро 5 & $1-3$ & 284808 & $9.08 \mathrm{E}+00$ & $9.08 \mathrm{E}+00$ & $0.00 \mathrm{E}+00$ & $5.70 \mathrm{E}-04$ \\
\hline $3-23$ & 3Po 2-3Do 5 & $3-7$ & 132.42 & $2.18 \mathrm{E}+00$ & $9.07 E+00$ & $2.65 E+05$ & $0.00 \mathrm{E}+00$ \\
\hline $4-23$ & 3Рo 2-3Do 5 & $5-7$ & 134.95 & $2.31 \mathrm{E}+00$ & $9.07 \mathrm{E}+00$ & $7.63 E+05$ & $1.04 \mathrm{E}+00$ \\
\hline $5-23$ & 1Po 2-3Do 5 & $3-7$ & 155.53 & $3.21 \mathrm{E}+00$ & $9.07 \mathrm{E}+00$ & $1.34 \mathrm{E}+02$ & $0.00 \mathrm{E}+00$ \\
\hline $15-23$ & 3Fo 5-3Do 5 & $5-7$ & 1501 & $8.46 \mathrm{E}+00$ & $9.07 \mathrm{E}+00$ & $9.71 \mathrm{E}-02$ & $1.21 \mathrm{E}+01$ \\
\hline $16-23$ & 3Fo 5-3Do 5 & $7-7$ & 1762 & $8.55 \mathrm{E}+00$ & $9.07 \mathrm{E}+00$ & $3.42 \mathrm{E}-01$ & $2.69 \mathrm{E}+01$ \\
\hline $17-23$ & 3Fo 5-3Do 5 & $9-7$ & 2212 & $8.65 E+00$ & $9.07 \mathrm{E}+00$ & $9.44 \mathrm{E}-02$ & $1.94 \mathrm{E}+01$ \\
\hline $18-23$ & 1Do 5-3Do 5 & $5-7$ & 2157 & $8.64 \mathrm{E}+00$ & $9.07 \mathrm{E}+00$ & $3.49 \mathrm{E}-02$ & $2.34 \mathrm{E}+00$ \\
\hline $19-23$ & 3Do 5-3Do 5 & $3-7$ & 8344 & $8.96 \mathrm{E}+00$ & $9.07 \mathrm{E}+00$ & $2.81 \mathrm{E}-04$ & $0.00 \mathrm{E}+00$ \\
\hline $20-23$ & 3Po 5-3Do 5 & $5-7$ & 8813 & $8.96 E+00$ & $9.07 E+00$ & $6.43 E-04$ & $1.15 \mathrm{E}+01$ \\
\hline $22-23$ & 3Po 5-3Do 5 & $3-7$ & 72323 & $9.08 \mathrm{E}+00$ & $9.07 \mathrm{E}+00$ & $1.01 \mathrm{E}-08$ & $0.00 \mathrm{E}+00$ \\
\hline $2-24$ & 3Рo 2-3Do 5 & $1-5$ & 131.10 & $2.13 \mathrm{E}+00$ & $9.08 \mathrm{E}+00$ & $1.17 \mathrm{E}+04$ & $0.00 \mathrm{E}+00$ \\
\hline $3-24$ & 3Рo 2-3Do 5 & $3-5$ & 132.11 & $2.18 \mathrm{E}+00$ & $9.08 \mathrm{E}+00$ & $4.22 \mathrm{E}+04$ & $6.40 \mathrm{E}+00$ \\
\hline $4-24$ & 3Рo 2-3Do 5 & $5-5$ & 134.63 & $2.31 \mathrm{E}+00$ & $9.08 \mathrm{E}+00$ & $9.73 E+05$ & $3.08 \mathrm{E}+00$ \\
\hline $5-24$ & 1Po 2-3Do 5 & $3-5$ & 155.11 & $3.21 \mathrm{E}+00$ & $9.08 \mathrm{E}+00$ & $4.90 \mathrm{E}+03$ & $7.41 \mathrm{E}+00$ \\
\hline $15-24$ & 3Fo 5-3Do 5 & $5-5$ & 1462 & $8.46 \mathrm{E}+00$ & $9.08 \mathrm{E}+00$ & $1.45 \mathrm{E}+00$ & $4.25 \mathrm{E}+01$ \\
\hline $16-24$ & 3Fo 5-3Do 5 & $7-5$ & 1709 & $8.55 \mathrm{E}+00$ & $9.08 \mathrm{E}+00$ & $1.87 \mathrm{E}+00$ & $1.57 \mathrm{E}+00$ \\
\hline $17-24$ & 3 Fo 5-3Do 5 & $9-5$ & 2129 & $8.65 E+00$ & $9.08 \mathrm{E}+00$ & $7.48 \mathrm{E}-01$ & $0.00 \mathrm{E}+00$ \\
\hline $18-24$ & 1Do 5-3Do 5 & $5-5$ & 2078 & $8.64 \mathrm{E}+00$ & $9.08 \mathrm{E}+00$ & $2.82 \mathrm{E}-02$ & $2.61 \mathrm{E}+01$ \\
\hline $19-24$ & 3Do 5-3Do 5 & $3-5$ & 7272 & $8.96 \mathrm{E}+00$ & $9.08 \mathrm{E}+00$ & $1.18 \mathrm{E}-03$ & $9.23 \mathrm{E}+00$ \\
\hline $20-24$ & 3Рo 5-3Do 5 & $5-5$ & 7625 & $8.96 \mathrm{E}+00$ & $9.08 \mathrm{E}+00$ & $4.90 \mathrm{E}-05$ & $9.50 \mathrm{E}+00$ \\
\hline $21-24$ & 3Рo 5-3Do 5 & $1-5$ & 136018 & $9.08 \mathrm{E}+00$ & $9.08 \mathrm{E}+00$ & $6.41 \mathrm{E}-10$ & $0.00 \mathrm{E}+00$ \\
\hline $22-24$ & 3Рo 5-3Do 5 & $3-5$ & 260363 & $9.08 \mathrm{E}+00$ & $9.08 \mathrm{E}+00$ & $2.12 \mathrm{E}-12$ & $8.99 E-04$ \\
\hline $23-24$ & 3Do 5-3Do 5 & $7-5$ & 56600 & $9.07 \mathrm{E}+00$ & $9.08 \mathrm{E}+00$ & $2.64 \mathrm{E}-08$ & $7.73 \mathrm{E}-02$ \\
\hline $3-25$ & 3Рo 2-1Fo 5 & $3-7$ & 121.53 & $2.18 \mathrm{E}+00$ & $9.68 \mathrm{E}+00$ & $5.31 \mathrm{E}+02$ & $0.00 \mathrm{E}+00$ \\
\hline $4-25$ & 3 Po $2-1$ Fo 5 & $5-7$ & 123.66 & $2.31 \mathrm{E}+00$ & $9.68 \mathrm{E}+00$ & $2.05 E+03$ & $1.01 \mathrm{E}+00$ \\
\hline $5-25$ & 1Po 2-1Fo 5 & $3-7$ & 140.72 & $3.21 \mathrm{E}+00$ & $9.68 \mathrm{E}+00$ & $4.75 E+05$ & $0.00 \mathrm{E}+00$ \\
\hline $15-25$ & 3Fo 5-1Fo 5 & $5-7$ & 744.74 & $8.46 \mathrm{E}+00$ & $9.68 \mathrm{E}+00$ & $4.87 \mathrm{E}+00$ & $8.93 \mathrm{E}+01$ \\
\hline $16-25$ & 3Fo 5-1Fo 5 & $7-7$ & 803.87 & $8.55 E+00$ & $9.68 \mathrm{E}+00$ & $3.25 \mathrm{E}-01$ & $1.66 \mathrm{E}+01$ \\
\hline $17-25$ & 3Fo 5-1Fo 5 & $9-7$ & 886.02 & $8.65 E+00$ & $9.68 \mathrm{E}+00$ & $3.22 \mathrm{E}-01$ & $5.05 \mathrm{E}+01$ \\
\hline $18-25$ & 1Do 5-1Fo 5 & $5-7$ & 877.15 & $8.64 \mathrm{E}+00$ & $9.68 \mathrm{E}+00$ & $1.23 \mathrm{E}+01$ & $1.00 \mathrm{E}+01$ \\
\hline $19-25$ & 3Do 5-1Fo 5 & $3-7$ & 1255 & $8.96 \mathrm{E}+00$ & $9.68 \mathrm{E}+00$ & $2.46 \mathrm{E}-01$ & $0.00 \mathrm{E}+00$ \\
\hline $20-25$ & 3Рo 5-1Fo 5 & $5-7$ & 1265 & $8.96 \mathrm{E}+00$ & $9.68 \mathrm{E}+00$ & $6.11 \mathrm{E}-03$ & $1.87 \mathrm{E}+01$ \\
\hline $22-25$ & 3Рo 5-1Fo 5 & $3-7$ & 1508 & $9.08 \mathrm{E}+00$ & $9.68 \mathrm{E}+00$ & $3.80 \mathrm{E}-04$ & $0.00 \mathrm{E}+00$ \\
\hline $23-25$ & 3Do 5-1Fo 5 & $7-7$ & 1477 & $9.07 \mathrm{E}+00$ & $9.68 \mathrm{E}+00$ & $1.75 \mathrm{E}-01$ & $6.08 \mathrm{E}+01$ \\
\hline $24-25$ & 3Do 5-1Fo 5 & $5-7$ & 1517 & $9.08 \mathrm{E}+00$ & $9.68 \mathrm{E}+00$ & $8.00 \mathrm{E}-03$ & $1.50 \mathrm{E}+01$ \\
\hline $2-26$ & 3Рo 2-1Po 5 & $1-3$ & 118.90 & $2.13 \mathrm{E}+00$ & $9.80 \mathrm{E}+00$ & $0.00 \mathrm{E}+00$ & $1.81 \mathrm{E}+01$ \\
\hline $3-26$ & 3Рo 2-1Po 5 & $3-3$ & 119.73 & $2.18 \mathrm{E}+00$ & $9.80 \mathrm{E}+00$ & $2.09 \mathrm{E}+02$ & $9.53 \mathrm{E}+00$ \\
\hline $4-26$ & 3Рo 2-1Po 5 & $5-3$ & 121.79 & $2.31 \mathrm{E}+00$ & $9.80 \mathrm{E}+00$ & $1.25 \mathrm{E}+02$ & $1.27 \mathrm{E}+01$ \\
\hline $5-26$ & 1Po 2-1Po 5 & $3-3$ & 138.32 & $3.21 \mathrm{E}+00$ & $9.80 \mathrm{E}+00$ & $1.23 \mathrm{E}+06$ & $4.83 \mathrm{E}-02$ \\
\hline $15-26$ & $3 F o$ 5-1Po 5 & $5-3$ & 681.93 & $8.46 \mathrm{E}+00$ & $9.80 \mathrm{E}+00$ & $4.86 \mathrm{E}+01$ & $1.12 \mathrm{E}+00$ \\
\hline $16-26$ & $3 F o$ 5-1Po 5 & $7-3$ & 731.18 & $8.55 \mathrm{E}+00$ & $9.80 \mathrm{E}+00$ & $7.73 E+00$ & $0.00 \mathrm{E}+00$ \\
\hline $18-26$ & 1Do 5-1Po 5 & $5-3$ & 791.31 & $8.64 \mathrm{E}+00$ & $9.80 \mathrm{E}+00$ & $2.16 \mathrm{E}+02$ & $1.37 \mathrm{E}+01$ \\
\hline $19-26$ & 3Do 5-1Po 5 & $3-3$ & 1086 & $8.96 \mathrm{E}+00$ & $9.80 \mathrm{E}+00$ & $9.34 \mathrm{E}-02$ & $1.61 \mathrm{E}+01$ \\
\hline $20-26$ & 3Рo 5-1Po 5 & $5-3$ & 1094 & $8.96 \mathrm{E}+00$ & $9.80 \mathrm{E}+00$ & $1.54 \mathrm{E}+00$ & $2.38 \mathrm{E}+02$ \\
\hline $21-26$ & 3Рo 5-1Po 5 & $1-3$ & 1265 & $9.08 \mathrm{E}+00$ & $9.80 \mathrm{E}+00$ & $0.00 \mathrm{E}+00$ & $3.23 E+01$ \\
\hline $22-26$ & 3Рo 5-1Po 5 & $3-3$ & 1271 & $9.08 \mathrm{E}+00$ & $9.80 \mathrm{E}+00$ & $7.07 \mathrm{E}-03$ & $5.93 E+01$ \\
\hline $23-26$ & 3Do 5-1Po 5 & $7-3$ & 1249 & $9.07 \mathrm{E}+00$ & $9.80 \mathrm{E}+00$ & $5.37 \mathrm{E}-02$ & $0.00 \mathrm{E}+00$ \\
\hline $24-26$ & 3Do 5-1Po 5 & $5-3$ & 1277 & $9.08 \mathrm{E}+00$ & $9.80 \mathrm{E}+00$ & $4.70 \mathrm{E}-01$ & $2.01 \mathrm{E}+01$ \\
\hline $25-26$ & 1Fo 5-1Po 5 & $7-3$ & 8085 & $9.68 \mathrm{E}+00$ & $9.80 \mathrm{E}+00$ & $5.54 \mathrm{E}-03$ & $0.00 \mathrm{E}+00$ \\
\hline $1-27$ & 1Se $1-3$ Fe 19 & $1-5$ & 72.98 & $0.00 \mathrm{E}+00$ & $1.25 \mathrm{E}+01$ & $7.51 \mathrm{E}+02$ & $0.00 \mathrm{E}+00$ \\
\hline $6-27$ & $3 \mathrm{Pe} 3-3 \mathrm{Fe} 19$ & $1-5$ & 122.58 & $5.05 \mathrm{E}+00$ & $1.25 \mathrm{E}+01$ & $1.01 \mathrm{E}+04$ & $0.00 \mathrm{E}+00$ \\
\hline $7-27$ & 1De 3-3Fe 19 & $5-5$ & 123.35 & $5.10 \mathrm{E}+00$ & $1.25 \mathrm{E}+01$ & $1.56 \mathrm{E}+03$ & $3.77 \mathrm{E}-02$ \\
\hline $8-27$ & 3Pe 3-3Fe 19 & $3-5$ & 124.12 & $5.14 \mathrm{E}+00$ & $1.25 \mathrm{E}+01$ & $9.65 E+03$ & $3.55 E-05$ \\
\hline $9-27$ & 3Pe 3-3Fe 19 & $5-5$ & 126.83 & $5.30 \mathrm{E}+00$ & $1.25 \mathrm{E}+01$ & $3.28 \mathrm{E}+03$ & $2.09 \mathrm{E}-03$ \\
\hline
\end{tabular}


Table 4. (continued)

\begin{tabular}{|c|c|c|c|c|c|c|c|}
\hline$i-j$ & $T_{i} C_{i}-T_{j} C_{j}$ & $g_{i}-g_{j}$ & $\lambda(\AA)$ & $E_{i}(\mathrm{Ry})$ & $E_{j}(\mathrm{Ry})$ & AE2 & AM1 \\
\hline $10-27$ & 1Se 3-3Fe 19 & $1-5$ & 140.71 & $6.01 \mathrm{E}+00$ & $1.25 \mathrm{E}+01$ & $4.34 \mathrm{E}+01$ & $0.00 \mathrm{E}+00$ \\
\hline $11-27$ & 3De 4-3Fe 19 & $3-5$ & 144.61 & $6.19 \mathrm{E}+00$ & $1.25 \mathrm{E}+01$ & $9.56 \mathrm{E}+05$ & $9.26 \mathrm{E}-01$ \\
\hline $12-27$ & 3De 4-3Fe 19 & $5-5$ & 144.82 & $6.19 \mathrm{E}+00$ & $1.25 \mathrm{E}+01$ & $6.87 \mathrm{E}+05$ & $1.66 \mathrm{E}-01$ \\
\hline $13-27$ & 3De 4-3Fe 19 & $7-5$ & 145.16 & $6.21 \mathrm{E}+00$ & $1.25 \mathrm{E}+01$ & $6.53 \mathrm{E}+04$ & $5.71 \mathrm{E}-02$ \\
\hline $14-27$ & 1De 4-3Fe 19 & $5-5$ & 164.42 & $6.94 \mathrm{E}+00$ & $1.25 \mathrm{E}+01$ & $2.71 \mathrm{E}+03$ & $2.44 \mathrm{E}+00$ \\
\hline $7-28$ & 1De 3-3Fe 19 & $5-7$ & 123.09 & $5.10 \mathrm{E}+00$ & $1.25 \mathrm{E}+01$ & $5.15 \mathrm{E}+02$ & $3.36 \mathrm{E}-01$ \\
\hline $8-28$ & 3Pe 3-3Fe 19 & $3-7$ & 123.85 & $5.14 \mathrm{E}+00$ & $1.25 \mathrm{E}+01$ & $1.41 \mathrm{E}+04$ & $0.00 \mathrm{E}+00$ \\
\hline $9-28$ & 3Pe 3-3Fe 19 & $5-7$ & 126.54 & $5.30 \mathrm{E}+00$ & $1.25 \mathrm{E}+01$ & $6.21 \mathrm{E}+03$ & $1.34 \mathrm{E}-01$ \\
\hline $11-28$ & 3De 4-3Fe 19 & $3-7$ & 144.24 & $6.19 \mathrm{E}+00$ & $1.25 \mathrm{E}+01$ & $3.48 \mathrm{E}+05$ & $0.00 \mathrm{E}+00$ \\
\hline $12-28$ & 3De 4-3Fe 19 & $5-7$ & 144.46 & $6.19 \mathrm{E}+00$ & $1.25 \mathrm{E}+01$ & $8.64 \mathrm{E}+05$ & $2.12 \mathrm{E}-01$ \\
\hline $13-28$ & 3De 4-3Fe 19 & $7-7$ & 144.79 & $6.21 \mathrm{E}+00$ & $1.25 \mathrm{E}+01$ & $5.13 \mathrm{E}+05$ & $6.04 \mathrm{E}-01$ \\
\hline $14-28$ & 1De 4-3Fe 19 & $5-7$ & 163.95 & $6.94 \mathrm{E}+00$ & $1.25 \mathrm{E}+01$ & $2.05 \mathrm{E}+02$ & $2.31 \mathrm{E}-01$ \\
\hline $27-28$ & $3 \mathrm{Fe} 19-3 \mathrm{Fe} 19$ & $5-7$ & 56954 & $1.25 \mathrm{E}+01$ & $1.25 \mathrm{E}+01$ & $4.57 \mathrm{E}-09$ & $1.38 \mathrm{E}-01$ \\
\hline $7-29$ & 1De 3-3Fe 19 & $5-9$ & 122.79 & $5.10 \mathrm{E}+00$ & $1.25 \mathrm{E}+01$ & $1.16 \mathrm{E}+04$ & $0.00 \mathrm{E}+00$ \\
\hline $9-29$ & 3Pe 3-3Fe 19 & $5-9$ & 126.23 & $5.30 \mathrm{E}+00$ & $1.25 \mathrm{E}+01$ & $1.15 \mathrm{E}+04$ & $0.00 \mathrm{E}+00$ \\
\hline $12-29$ & 3De 4-3Fe 19 & $5-9$ & 144.04 & $6.19 \mathrm{E}+00$ & $1.25 \mathrm{E}+01$ & $2.93 \mathrm{E}+05$ & $0.00 \mathrm{E}+00$ \\
\hline $13-29$ & 3De 4-3Fe 19 & $7-9$ & 144.38 & $6.21 \mathrm{E}+00$ & $1.25 \mathrm{E}+01$ & $1.44 \mathrm{E}+06$ & $2.88 \mathrm{E}-01$ \\
\hline $14-29$ & 1De 4-3Fe 19 & $5-9$ & 163.42 & $6.94 \mathrm{E}+00$ & $1.25 \mathrm{E}+01$ & $8.15 \mathrm{E}+02$ & $0.00 \mathrm{E}+00$ \\
\hline $27-29$ & 3Fe 19-3Fe 19 & $5-9$ & 26802 & $1.25 \mathrm{E}+01$ & $1.25 \mathrm{E}+01$ & $5.29 \mathrm{E}-09$ & $0.00 \mathrm{E}+00$ \\
\hline $28-29$ & 3Fe 19-3Fe 19 & $7-9$ & 50626 & $1.25 \mathrm{E}+01$ & $1.25 \mathrm{E}+01$ & $6.56 \mathrm{E}-09$ & $1.56 \mathrm{E}-01$ \\
\hline $1-30$ & 1Se $1-3 \mathrm{Pe} 19$ & $1-5$ & 71.03 & $0.00 \mathrm{E}+00$ & $1.28 \mathrm{E}+01$ & $1.11 \mathrm{E}+05$ & $0.00 \mathrm{E}+00$ \\
\hline $6-30$ & 3Рe 3-3Рe 19 & $1-5$ & 117.19 & $5.05 \mathrm{E}+00$ & $1.28 \mathrm{E}+01$ & $5.86 \mathrm{E}+00$ & $0.00 \mathrm{E}+00$ \\
\hline $7-30$ & 1De 3-3Pe 19 & $5-5$ & 117.89 & $5.10 \mathrm{E}+00$ & $1.28 \mathrm{E}+01$ & $4.30 \mathrm{E}+05$ & $3.40 \mathrm{E}-02$ \\
\hline $8-30$ & 3Рe 3-3Pe 19 & $3-5$ & 118.59 & $5.14 \mathrm{E}+00$ & $1.28 \mathrm{E}+01$ & $2.39 \mathrm{E}+01$ & $1.77 \mathrm{E}-02$ \\
\hline $9-30$ & 3Рe 3-3Pe 19 & $5-5$ & 121.06 & $5.30 \mathrm{E}+00$ & $1.28 \mathrm{E}+01$ & $8.03 E+04$ & $7.19 \mathrm{E}+00$ \\
\hline $10-30$ & 1Se 3-3Pe 19 & $1-5$ & 133.66 & $6.01 \mathrm{E}+00$ & $1.28 \mathrm{E}+01$ & $7.91 \mathrm{E}+03$ & $0.00 \mathrm{E}+00$ \\
\hline $11-30$ & 3De 4-3Pe 19 & $3-5$ & 137.16 & $6.19 \mathrm{E}+00$ & $1.28 \mathrm{E}+01$ & $8.99 \mathrm{E}+04$ & $1.14 \mathrm{E}+00$ \\
\hline $12-30$ & 3De 4-3Pe 19 & $5-5$ & 137.36 & $6.19 \mathrm{E}+00$ & $1.28 \mathrm{E}+01$ & $1.80 \mathrm{E}+05$ & $2.91 \mathrm{E}-01$ \\
\hline $13-30$ & 3De 4-3Pe 19 & $7-5$ & 137.66 & $6.21 \mathrm{E}+00$ & $1.28 \mathrm{E}+01$ & $3.55 \mathrm{E}+05$ & $1.64 \mathrm{E}-02$ \\
\hline $14-30$ & 1De 4-3Pe 19 & $5-5$ & 154.86 & $6.94 \mathrm{E}+00$ & $1.28 \mathrm{E}+01$ & $8.88 \mathrm{E}+05$ & $4.10 \mathrm{E}-02$ \\
\hline $27-30$ & 3Fe 19-3Pe 19 & $5-5$ & 2664 & $1.25 \mathrm{E}+01$ & $1.28 \mathrm{E}+01$ & $1.15 \mathrm{E}-02$ & $3.62 \mathrm{E}+00$ \\
\hline $28-30$ & 3Fe 19-3Pe 19 & $7-5$ & 2795 & $1.25 \mathrm{E}+01$ & $1.28 \mathrm{E}+01$ & $3.38 \mathrm{E}-02$ & $5.84 \mathrm{E}+00$ \\
\hline $29-30$ & 3Fe 19-3Pe 19 & $9-5$ & 2958 & $1.25 \mathrm{E}+01$ & $1.28 \mathrm{E}+01$ & $1.21 \mathrm{E}-01$ & $0.00 \mathrm{E}+00$ \\
\hline $7-31$ & 1De 3-3Pe 19 & $5-1$ & 115.72 & $5.10 \mathrm{E}+00$ & $1.30 \mathrm{E}+01$ & $8.75 \mathrm{E}+02$ & $0.00 \mathrm{E}+00$ \\
\hline $8-31$ & 3Рe 3-3Рe 19 & $3-1$ & 116.39 & $5.14 \mathrm{E}+00$ & $1.30 \mathrm{E}+01$ & $0.00 \mathrm{E}+00$ & $7.83 \mathrm{E}+00$ \\
\hline $9-31$ & 3Рe 3-3Pe 19 & $5-1$ & 118.77 & $5.30 \mathrm{E}+00$ & $1.30 \mathrm{E}+01$ & $2.04 \mathrm{E}+03$ & $0.00 \mathrm{E}+00$ \\
\hline $11-31$ & 3De 4-3Pe 19 & $3-1$ & 134.22 & $6.19 \mathrm{E}+00$ & $1.30 \mathrm{E}+01$ & $0.00 \mathrm{E}+00$ & $1.35 \mathrm{E}+00$ \\
\hline $12-31$ & 3De 4-3Pe 19 & $5-1$ & 134.41 & $6.19 \mathrm{E}+00$ & $1.30 \mathrm{E}+01$ & $2.45 \mathrm{E}+06$ & $0.00 \mathrm{E}+00$ \\
\hline $14-31$ & 1De 4-3Pe 19 & $5-1$ & 151.13 & $6.94 \mathrm{E}+00$ & $1.30 \mathrm{E}+01$ & $7.55 \mathrm{E}+02$ & $0.00 \mathrm{E}+00$ \\
\hline $27-31$ & 3Fe 19-3Pe 19 & $5-1$ & 1868 & $1.25 \mathrm{E}+01$ & $1.30 \mathrm{E}+01$ & $5.12 \mathrm{E}+00$ & $0.00 \mathrm{E}+00$ \\
\hline $30-31$ & 3Рe 19-3Рe 19 & $5-1$ & 6260 & $1.28 \mathrm{E}+01$ & $1.30 \mathrm{E}+01$ & $2.97 \mathrm{E}-03$ & $0.00 \mathrm{E}+00$ \\
\hline $1-32$ & 1Se 1-3Pe 19 & $1-3$ & 70.19 & $0.00 \mathrm{E}+00$ & $1.30 \mathrm{E}+01$ & $0.00 \mathrm{E}+00$ & $1.77 \mathrm{E}+00$ \\
\hline $6-32$ & 3Рe 3-3Рe 19 & $1-3$ & 114.91 & $5.05 \mathrm{E}+00$ & $1.30 \mathrm{E}+01$ & $0.00 \mathrm{E}+00$ & $2.36 \mathrm{E}+00$ \\
\hline $7-32$ & 1De 3-3Pe 19 & $5-3$ & 115.58 & $5.10 \mathrm{E}+00$ & $1.30 \mathrm{E}+01$ & $4.93 \mathrm{E}+01$ & $3.72 \mathrm{E}-02$ \\
\hline $8-32$ & 3Рe 3-3Рe 19 & $3-3$ & 116.25 & $5.14 \mathrm{E}+00$ & $1.30 \mathrm{E}+01$ & $2.60 \mathrm{E}+02$ & $7.86 \mathrm{E}-04$ \\
\hline $9-32$ & 3Рe 3-3Рe 19 & $5-3$ & 118.63 & $5.30 \mathrm{E}+00$ & $1.30 \mathrm{E}+01$ & $5.85 \mathrm{E}+02$ & $9.76 \mathrm{E}+00$ \\
\hline $10-32$ & 1Se 3-3Pe 19 & $1-3$ & 130.69 & $6.01 E+00$ & $1.30 \mathrm{E}+01$ & $0.00 \mathrm{E}+00$ & $1.85 E+00$ \\
\hline $11-32$ & 3De 4-3Pe 19 & $3-3$ & 134.04 & $6.19 \mathrm{E}+00$ & $1.30 \mathrm{E}+01$ & $1.12 \mathrm{E}+06$ & $5.77 \mathrm{E}-06$ \\
\hline $12-32$ & 3De 4-3Pe 19 & $5-3$ & 134.23 & $6.19 \mathrm{E}+00$ & $1.30 \mathrm{E}+01$ & $2.06 \mathrm{E}+05$ & $3.84 \mathrm{E}-01$ \\
\hline $13-32$ & 3De 4-3Pe 19 & $7-3$ & 134.52 & $6.21 \mathrm{E}+00$ & $1.30 \mathrm{E}+01$ & $1.14 \mathrm{E}+06$ & $0.00 \mathrm{E}+00$ \\
\hline $14-32$ & 1De 4-3Pe 19 & $5-3$ & 150.90 & $6.94 \mathrm{E}+00$ & $1.30 \mathrm{E}+01$ & $4.14 \mathrm{E}+01$ & $3.69 \mathrm{E}-01$ \\
\hline $27-32$ & 3Fe 19-3Pe 19 & $5-3$ & 1834 & $1.25 \mathrm{E}+01$ & $1.30 \mathrm{E}+01$ & $1.82 \mathrm{E}+00$ & $4.78 \mathrm{E}-02$ \\
\hline $28-32$ & 3Fe 19-3Pe 19 & $7-3$ & 1895 & $1.25 \mathrm{E}+01$ & $1.30 \mathrm{E}+01$ & $3.14 \mathrm{E}+00$ & $0.00 \mathrm{E}+00$ \\
\hline $30-32$ & 3Рe 19-3Рe 19 & $5-3$ & 5892 & $1.28 \mathrm{E}+01$ & $1.30 \mathrm{E}+01$ & $3.46 \mathrm{E}-03$ & $3.02 \mathrm{E}+01$ \\
\hline $31-32$ & 3Ре 19-3Ре 19 & $1-3$ & 100497 & $1.30 \mathrm{E}+01$ & $1.30 \mathrm{E}+01$ & $0.00 \mathrm{E}+00$ & $1.77 \mathrm{E}-02$ \\
\hline $1-33$ & 1Se 1-1De 19 & $1-5$ & 71.30 & $0.00 \mathrm{E}+00$ & $1.28 \mathrm{E}+01$ & $4.06 \mathrm{E}+04$ & $0.00 \mathrm{E}+00$ \\
\hline $6-33$ & 3Pe 3-1De 19 & $1-5$ & 117.92 & $5.05 \mathrm{E}+00$ & $1.28 \mathrm{E}+01$ & $3.66 \mathrm{E}+02$ & $0.00 \mathrm{E}+00$ \\
\hline $7-33$ & 1De 3-1De 19 & $5-5$ & 118.63 & $5.10 \mathrm{E}+00$ & $1.28 \mathrm{E}+01$ & $1.43 \mathrm{E}+05$ & $5.99 \mathrm{E}+00$ \\
\hline $8-33$ & 3Pe 3-1De 19 & $3-5$ & 119.34 & $5.14 \mathrm{E}+00$ & $1.28 \mathrm{E}+01$ & $4.42 \mathrm{E}+02$ & $6.66 \mathrm{E}+00$ \\
\hline $9-33$ & 3Pe 3-1De 19 & $5-5$ & 121.84 & $5.30 \mathrm{E}+00$ & $1.28 \mathrm{E}+01$ & $3.35 \mathrm{E}+04$ & $2.77 \mathrm{E}-01$ \\
\hline $10-33$ & 1Se 3-1De 19 & $1-5$ & 134.60 & $6.01 \mathrm{E}+00$ & $1.28 \mathrm{E}+01$ & $2.61 \mathrm{E}+03$ & $0.00 \mathrm{E}+00$ \\
\hline $11-33$ & 3De 4-1De 19 & $3-5$ & 138.16 & $6.19 \mathrm{E}+00$ & $1.28 \mathrm{E}+01$ & $1.15 \mathrm{E}+05$ & $1.28 \mathrm{E}-01$ \\
\hline $12-33$ & 3De 4-1De 19 & $5-5$ & 138.36 & $6.19 \mathrm{E}+00$ & $1.28 \mathrm{E}+01$ & $5.64 \mathrm{E}+05$ & $1.10 \mathrm{E}-01$ \\
\hline $13-33$ & 3De 4-1De 19 & $7-5$ & 138.67 & $6.21 \mathrm{E}+00$ & $1.28 \mathrm{E}+01$ & $8.36 \mathrm{E}+05$ & $2.12 \mathrm{E}-01$ \\
\hline $14-33$ & 1De 4-1De 19 & $5-5$ & 156.14 & $6.94 \mathrm{E}+00$ & $1.28 \mathrm{E}+01$ & $3.39 \mathrm{E}+05$ & $3.77 \mathrm{E}-02$ \\
\hline $27-33$ & 3Fe 19-1De 19 & $5-5$ & 3099 & $1.25 \mathrm{E}+01$ & $1.28 \mathrm{E}+01$ & $6.91 \mathrm{E}-03$ & $4.24 \mathrm{E}-01$ \\
\hline $28-33$ & 3Fe 19-1De 19 & $7-5$ & 3277 & $1.25 \mathrm{E}+01$ & $1.28 \mathrm{E}+01$ & $4.56 \mathrm{E}-02$ & $1.01 \mathrm{E}+00$ \\
\hline $29-33$ & 3Fe 19-1De 19 & $9-5$ & 3504 & $1.25 \mathrm{E}+01$ & $1.28 \mathrm{E}+01$ & $1.16 \mathrm{E}-01$ & $0.00 \mathrm{E}+00$ \\
\hline $30-33$ & 3Pe 19-1De 19 & $5-5$ & 18984 & $1.28 \mathrm{E}+01$ & $1.28 \mathrm{E}+01$ & $1.26 \mathrm{E}-06$ & $1.18 \mathrm{E}+00$ \\
\hline $31-33$ & 3Pe 19-1De 19 & $1-5$ & 4707 & $1.30 \mathrm{E}+01$ & $1.28 \mathrm{E}+01$ & $6.34 \mathrm{E}-03$ & $0.00 \mathrm{E}+00$ \\
\hline $32-33$ & 3Pe 19-1De 19 & $3-5$ & 4497 & $1.30 \mathrm{E}+01$ & $1.28 \mathrm{E}+01$ & $1.73 \mathrm{E}-02$ & $1.08 \mathrm{E}+02$ \\
\hline $7-34$ & 1De 3-1Ge 19 & $5-9$ & 118.00 & $5.10 \mathrm{E}+00$ & $1.28 \mathrm{E}+01$ & $1.20 \mathrm{E}+06$ & $0.00 \mathrm{E}+00$ \\
\hline $9-34$ & 3Pe 3-1Ge 19 & $5-9$ & 121.18 & $5.30 \mathrm{E}+00$ & $1.28 \mathrm{E}+01$ & $2.21 \mathrm{E}+05$ & $0.00 \mathrm{E}+00$ \\
\hline $12-34$ & 3De 4-1Ge 19 & $5-9$ & 137.50 & $6.19 \mathrm{E}+00$ & $1.28 \mathrm{E}+01$ & $1.10 \mathrm{E}+02$ & $0.00 \mathrm{E}+00$ \\
\hline $13-34$ & 3De $4-1 \mathrm{Ge} 19$ & $7-9$ & 137.81 & $6.21 \mathrm{E}+00$ & $1.28 \mathrm{E}+01$ & $3.75 E+03$ & $9.88 \mathrm{E}-01$ \\
\hline $14-34$ & 1De 4-1Ge 19 & $5-9$ & 155.05 & $6.94 \mathrm{E}+00$ & $1.28 \mathrm{E}+01$ & $7.65 \mathrm{E}+05$ & $0.00 \mathrm{E}+00$ \\
\hline
\end{tabular}


Table 4. (continued)

\begin{tabular}{|c|c|c|c|c|c|c|c|}
\hline$i-j$ & $T_{i} C_{i}-T_{j} C_{j}$ & $g_{i}-g_{j}$ & $\lambda(\AA)$ & $E_{i}(\mathrm{Ry})$ & $E_{j}(\mathrm{Ry})$ & AE2 & AM1 \\
\hline $27-34$ & 3Fe $19-1 \mathrm{Ge} 19$ & $5-9$ & 2720 & $1.25 \mathrm{E}+01$ & $1.28 \mathrm{E}+01$ & $9.94 \mathrm{E}-04$ & $0.00 \mathrm{E}+00$ \\
\hline $28-34$ & 3Fe 19-1Ge 19 & $7-9$ & 2856 & $1.25 \mathrm{E}+01$ & $1.28 \mathrm{E}+01$ & $2.37 \mathrm{E}-05$ & $1.75 E+00$ \\
\hline $29-34$ & 3Fe 19-1Ge 19 & $9-9$ & 3027 & $1.25 \mathrm{E}+01$ & $1.28 \mathrm{E}+01$ & $7.57 \mathrm{E}-04$ & $2.52 \mathrm{E}+00$ \\
\hline $30-34$ & 3Pe 19-1Ge 19 & $5-9$ & 130163 & $1.28 \mathrm{E}+01$ & $1.28 \mathrm{E}+01$ & $5.47 \mathrm{E}-10$ & $0.00 \mathrm{E}+00$ \\
\hline $33-34$ & 1De 19-1Ge 19 & $5-9$ & 22226 & $1.28 \mathrm{E}+01$ & $1.28 \mathrm{E}+01$ & $1.83 \mathrm{E}-06$ & $0.00 \mathrm{E}+00$ \\
\hline $7-35$ & 1De 3-1Se 19 & $5-1$ & 107.82 & $5.10 \mathrm{E}+00$ & $1.36 \mathrm{E}+01$ & $3.95 \mathrm{E}+05$ & $0.00 \mathrm{E}+00$ \\
\hline $8-35$ & 3Pe 3-1Se 19 & $3-1$ & 108.41 & $5.14 \mathrm{E}+00$ & $1.36 \mathrm{E}+01$ & $0.00 \mathrm{E}+00$ & $2.12 \mathrm{E}+02$ \\
\hline 9-35 & 3Pe 3-1Se 19 & $5-1$ & 110.47 & $5.30 \mathrm{E}+00$ & $1.36 \mathrm{E}+01$ & $8.60 \mathrm{E}+04$ & $0.00 \mathrm{E}+00$ \\
\hline $11-35$ & 3De 4-1Se 19 & $3-1$ & 123.72 & $6.19 \mathrm{E}+00$ & $1.36 \mathrm{E}+01$ & $0.00 \mathrm{E}+00$ & $7.16 \mathrm{E}-01$ \\
\hline $12-35$ & 3De 4-1Se 19 & $5-1$ & 123.88 & $6.19 \mathrm{E}+00$ & $1.36 \mathrm{E}+01$ & $2.61 \mathrm{E}+03$ & $0.00 \mathrm{E}+00$ \\
\hline $14-35$ & 1De 4-1Se 19 & $5-1$ & 137.94 & $6.94 \mathrm{E}+00$ & $1.36 \mathrm{E}+01$ & $2.16 \mathrm{E}+06$ & $0.00 \mathrm{E}+00$ \\
\hline $27-35$ & 3Fe 19-1Se 19 & $5-1$ & 856.45 & $1.25 \mathrm{E}+01$ & $1.36 \mathrm{E}+01$ & $1.11 \mathrm{E}+00$ & $0.00 \mathrm{E}+00$ \\
\hline $30-35$ & 3Pe 19-1Se 19 & $5-1$ & 1262 & $1.28 \mathrm{E}+01$ & $1.36 \mathrm{E}+01$ & $6.94 \mathrm{E}+01$ & $0.00 \mathrm{E}+00$ \\
\hline $32-35$ & 3Pe 19-1Se 19 & $3-1$ & 1606 & $1.30 \mathrm{E}+01$ & $1.36 \mathrm{E}+01$ & $0.00 \mathrm{E}+00$ & $2.61 \mathrm{E}+01$ \\
\hline $33-35$ & 1De 19-1Se 19 & $5-1$ & 1183 & $1.28 \mathrm{E}+01$ & $1.36 \mathrm{E}+01$ & $4.05 \mathrm{E}+01$ & $0.00 \mathrm{E}+00$ \\
\hline $1-36$ & 1Se 1-3Se 6 & $1-3$ & 56.70 & $0.00 \mathrm{E}+00$ & $1.61 \mathrm{E}+01$ & $0.00 \mathrm{E}+00$ & $3.27 \mathrm{E}+01$ \\
\hline $6-36$ & 3Pe 3-3Se 6 & $1-3$ & 82.70 & $5.05 \mathrm{E}+00$ & $1.61 \mathrm{E}+01$ & $0.00 \mathrm{E}+00$ & $9.55 \mathrm{E}+00$ \\
\hline $7-36$ & 1De 3-3Se 6 & $5-3$ & 83.05 & $5.10 \mathrm{E}+00$ & $1.61 \mathrm{E}+01$ & $1.05 E+00$ & $7.76 \mathrm{E}-01$ \\
\hline $8-36$ & 3Pe 3-3Se 6 & $3-3$ & 83.40 & $5.14 \mathrm{E}+00$ & $1.61 \mathrm{E}+01$ & $8.96 \mathrm{E}+00$ & $7.99 \mathrm{E}+00$ \\
\hline $9-36$ & 3Pe 3-3Se 6 & $5-3$ & 84.61 & $5.30 \mathrm{E}+00$ & $1.61 \mathrm{E}+01$ & $8.05 E+01$ & $6.06 \mathrm{E}+00$ \\
\hline $10-36$ & 1Se 3-3Se 6 & $1-3$ & 90.57 & $6.01 \mathrm{E}+00$ & $1.61 \mathrm{E}+01$ & $0.00 \mathrm{E}+00$ & $2.96 \mathrm{E}-02$ \\
\hline $11-36$ & 3De 4-3Se 6 & $3-3$ & 92.17 & $6.19 \mathrm{E}+00$ & $1.61 \mathrm{E}+01$ & $3.27 \mathrm{E}+06$ & $2.18 \mathrm{E}-02$ \\
\hline $12-36$ & 3De 4-3Se 6 & $5-3$ & 92.26 & $6.19 \mathrm{E}+00$ & $1.61 \mathrm{E}+01$ & $5.46 \mathrm{E}+06$ & $6.47 \mathrm{E}-02$ \\
\hline $13-36$ & 3De 4-3Se 6 & $7-3$ & 92.40 & $6.21 \mathrm{E}+00$ & $1.61 \mathrm{E}+01$ & $7.67 \mathrm{E}+06$ & $0.00 \mathrm{E}+00$ \\
\hline $14-36$ & 1De 4-3Se 6 & $5-3$ & 99.84 & $6.94 \mathrm{E}+00$ & $1.61 \mathrm{E}+01$ & $1.58 \mathrm{E}+03$ & $8.55 \mathrm{E}-03$ \\
\hline $27-36$ & 3Fe 19-3Se 6 & $5-3$ & 254.19 & $1.25 \mathrm{E}+01$ & $1.61 \mathrm{E}+01$ & $3.88 \mathrm{E}-03$ & $2.11 \mathrm{E}-07$ \\
\hline $28-36$ & 3Fe 19-3Se 6 & $7-3$ & 255.33 & $1.25 \mathrm{E}+01$ & $1.61 \mathrm{E}+01$ & $5.98 \mathrm{E}-03$ & $0.00 \mathrm{E}+00$ \\
\hline $30-36$ & 3Pe 19-3Se 6 & $5-3$ & 281.00 & $1.28 \mathrm{E}+01$ & $1.61 \mathrm{E}+01$ & $7.47 \mathrm{E}-03$ & $4.40 \mathrm{E}-03$ \\
\hline $31-36$ & 3Pe 19-3Se 6 & $1-3$ & 294.20 & $1.30 \mathrm{E}+01$ & $1.61 \mathrm{E}+01$ & $0.00 \mathrm{E}+00$ & $1.36 \mathrm{E}-02$ \\
\hline $32-36$ & 3Pe 19-3Se 6 & $3-3$ & 295.06 & $1.30 \mathrm{E}+01$ & $1.61 \mathrm{E}+01$ & $3.17 \mathrm{E}-04$ & $1.03 \mathrm{E}-02$ \\
\hline $33-36$ & 1De 19-3Se 6 & $5-3$ & 276.90 & $1.28 \mathrm{E}+01$ & $1.61 \mathrm{E}+01$ & $1.35 \mathrm{E}-04$ & $1.27 \mathrm{E}-02$ \\
\hline $35-36$ & 1Se 19-3Se 6 & $1-3$ & 361.47 & $1.36 \mathrm{E}+01$ & $1.61 \mathrm{E}+01$ & $0.00 \mathrm{E}+00$ & $5.30 \mathrm{E}-04$ \\
\hline $7-37$ & 1De 3-1Se 6 & $5-1$ & 81.48 & $5.10 \mathrm{E}+00$ & $1.63 \mathrm{E}+01$ & $8.85 \mathrm{E}+06$ & $0.00 \mathrm{E}+00$ \\
\hline $8-37$ & 3Pe 3-1Se 6 & $3-1$ & 81.81 & $5.14 \mathrm{E}+00$ & $1.63 \mathrm{E}+01$ & $0.00 \mathrm{E}+00$ & $3.12 \mathrm{E}+01$ \\
\hline $9-37$ & 3Pe 3-1Se 6 & $5-1$ & 82.98 & $5.30 \mathrm{E}+00$ & $1.63 \mathrm{E}+01$ & $1.61 \mathrm{E}+06$ & $0.00 \mathrm{E}+00$ \\
\hline $11-37$ & 3De 4-1Se 6 & $3-1$ & 90.24 & $6.19 \mathrm{E}+00$ & $1.63 \mathrm{E}+01$ & $0.00 \mathrm{E}+00$ & $9.51 \mathrm{E}-02$ \\
\hline$i-j$ & $T_{i} C_{i}-T_{j} C_{j}$ & $g_{i}-g_{j}$ & $\lambda(\AA)$ & $E_{i}(\mathrm{Ry})$ & $E_{j}(\mathrm{Ry})$ & AE3 & AM2 \\
\hline \multicolumn{8}{|c|}{ E3 and $M 2, N_{t r}=1896$} \\
\hline $1-4$ & 1Se $1-3$ Po 2 & $1-5$ & 393.98 & $0.00 \mathrm{E}+00$ & $2.31 \mathrm{E}+00$ & $0.00 \mathrm{E}+00$ & $3.38 \mathrm{E}+00$ \\
\hline $4-6$ & 3Рo 2-3Pe 3 & $5-1$ & 332.55 & $2.31 \mathrm{E}+00$ & $5.05 \mathrm{E}+00$ & $0.00 \mathrm{E}+00$ & $2.99 \mathrm{E}+00$ \\
\hline $2-7$ & 3Po 2-1De 3 & $1-5$ & 306.97 & $2.13 \mathrm{E}+00$ & $5.10 \mathrm{E}+00$ & $0.00 \mathrm{E}+00$ & $6.54 \mathrm{E}+00$ \\
\hline $2-9$ & 3 Po 2-3Pe 3 & $1-5$ & 287.38 & $2.13 E+00$ & $5.30 \mathrm{E}+00$ & $0.00 \mathrm{E}+00$ & $6.08 \mathrm{E}-02$ \\
\hline $4-10$ & 3Po 2-1Se 3 & $5-1$ & 246.42 & $2.31 \mathrm{E}+00$ & $6.01 \mathrm{E}+00$ & $0.00 \mathrm{E}+00$ & $4.03 \mathrm{E}+01$ \\
\hline $2-12$ & 3Po 2-3De 4 & $1-5$ & 224.24 & $2.13 \mathrm{E}+00$ & $6.19 \mathrm{E}+00$ & $0.00 \mathrm{E}+00$ & $3.19 \mathrm{E}+01$ \\
\hline $2-13$ & 3Po 2-3De 4 & $1-7$ & 223.43 & $2.13 E+00$ & $6.21 \mathrm{E}+00$ & $1.63 \mathrm{E}-01$ & $0.00 \mathrm{E}+00$ \\
\hline $3-13$ & 3Po 2-3De 4 & $3-7$ & 226.37 & $2.18 \mathrm{E}+00$ & $6.21 \mathrm{E}+00$ & $2.97 \mathrm{E}-01$ & $2.63 E+01$ \\
\hline $5-13$ & 1Po 2-3De 4 & $3-7$ & 303.48 & $3.21 \mathrm{E}+00$ & $6.21 \mathrm{E}+00$ & $2.60 \mathrm{E}-04$ & $7.88 \mathrm{E}+00$ \\
\hline $2-14$ & 3Po 2-1De 4 & $1-5$ & 189.30 & $2.13 \mathrm{E}+00$ & $6.94 \mathrm{E}+00$ & $0.00 \mathrm{E}+00$ & $1.32 \mathrm{E}+00$ \\
\hline $1-15$ & 1Se $1-3$ Fo 5 & $1-5$ & 107.73 & $0.00 \mathrm{E}+00$ & $8.46 \mathrm{E}+00$ & $0.00 \mathrm{E}+00$ & $1.15 \mathrm{E}+00$ \\
\hline $6-15$ & 3Pe $3-3$ Fo 5 & $1-5$ & 267.58 & $5.05 \mathrm{E}+00$ & $8.46 \mathrm{E}+00$ & $0.00 \mathrm{E}+00$ & $6.38 \mathrm{E}+00$ \\
\hline $10-15$ & 1Se $3-3$ Fo 5 & $1-5$ & 372.28 & $6.01 \mathrm{E}+00$ & $8.46 \mathrm{E}+00$ & $0.00 \mathrm{E}+00$ & $4.06 \mathrm{E}-02$ \\
\hline $1-16$ & 1Se $1-3$ Fo 5 & $1-7$ & 106.60 & $0.00 \mathrm{E}+00$ & $8.55 \mathrm{E}+00$ & $1.31 \mathrm{E}-02$ & $0.00 \mathrm{E}+00$ \\
\hline $6-16$ & 3Pe 3-3Fo 5 & $1-7$ & 260.69 & $5.05 \mathrm{E}+00$ & $8.55 E+00$ & $7.10 \mathrm{E}-02$ & $0.00 \mathrm{E}+00$ \\
\hline $8-16$ & 3Pe 3-3Fo 5 & $3-7$ & 267.72 & $5.14 \mathrm{E}+00$ & $8.55 E+00$ & $1.28 \mathrm{E}-02$ & $4.23 \mathrm{E}+00$ \\
\hline $10-16$ & 1Se 3-3Fo 5 & $1-7$ & 359.08 & $6.01 \mathrm{E}+00$ & $8.55 \mathrm{E}+00$ & $1.28 \mathrm{E}-04$ & $0.00 \mathrm{E}+00$ \\
\hline $11-16$ & 3De 4-3Fo 5 & $3-7$ & 385.57 & $6.19 \mathrm{E}+00$ & $8.55 \mathrm{E}+00$ & $5.31 \mathrm{E}-03$ & $1.36 \mathrm{E}+00$ \\
\hline $7-17$ & 1De 3-3Fo 5 & $5-9$ & 256.38 & $5.10 \mathrm{E}+00$ & $8.65 E+00$ & $1.95 \mathrm{E}-02$ & $4.41 \mathrm{E}+01$ \\
\hline $8-17$ & 3Pe 3-3Fo 5 & $3-9$ & 259.70 & $5.14 \mathrm{E}+00$ & $8.65 \mathrm{E}+00$ & $6.88 \mathrm{E}-02$ & $0.00 \mathrm{E}+00$ \\
\hline $9-17$ & 3Pe 3-3Fo 5 & $5-9$ & 271.85 & $5.30 \mathrm{E}+00$ & $8.65 E+00$ & $7.15 \mathrm{E}-02$ & $1.43 \mathrm{E}-02$ \\
\hline $11-17$ & 3De 4-3Fo 5 & $3-9$ & 369.16 & $6.19 \mathrm{E}+00$ & $8.65 \mathrm{E}+00$ & $9.03 \mathrm{E}-04$ & $0.00 \mathrm{E}+00$ \\
\hline $12-17$ & 3De 4-3Fo 5 & $5-9$ & 370.55 & $6.19 E+00$ & $8.65 E+00$ & $7.35 \mathrm{E}-03$ & $1.18 \mathrm{E}+00$ \\
\hline $14-17$ & 1De 4-3Fo 5 & $5-9$ & 533.15 & $6.94 \mathrm{E}+00$ & $8.65 E+00$ & $3.17 \mathrm{E}-09$ & $1.74 \mathrm{E}-02$ \\
\hline $1-18$ & 1Se 1-1Do 5 & $1-5$ & 105.43 & $0.00 \mathrm{E}+00$ & $8.64 \mathrm{E}+00$ & $0.00 \mathrm{E}+00$ & $9.43 \mathrm{E}+00$ \\
\hline $6-18$ & 3Pe 3-1Do 5 & $1-5$ & 253.81 & $5.05 \mathrm{E}+00$ & $8.64 \mathrm{E}+00$ & $0.00 \mathrm{E}+00$ & $6.74 \mathrm{E}+00$ \\
\hline $10-18$ & 1Se 3-1Do 5 & $1-5$ & 346.16 & $6.01 \mathrm{E}+00$ & $8.64 \mathrm{E}+00$ & $0.00 \mathrm{E}+00$ & $1.58 \mathrm{E}+00$ \\
\hline $13-19$ & 3De 4-3Do 5 & $7-3$ & 331.72 & $6.21 \mathrm{E}+00$ & $8.96 \mathrm{E}+00$ & $4.18 \mathrm{E}-02$ & $8.24 \mathrm{E}-02$ \\
\hline $1-20$ & 1Se $1-3$ Po 5 & $1-5$ & 101.68 & $0.00 \mathrm{E}+00$ & $8.96 \mathrm{E}+00$ & $0.00 \mathrm{E}+00$ & $5.31 \mathrm{E}-01$ \\
\hline $6-20$ & 3Pe 3-3Po 5 & $1-5$ & 233.11 & $5.05 \mathrm{E}+00$ & $8.96 \mathrm{E}+00$ & $0.00 \mathrm{E}+00$ & $1.29 \mathrm{E}-03$ \\
\hline $10-20$ & 1Se 3-3Po 5 & $1-5$ & 308.76 & $6.01 \mathrm{E}+00$ & $8.96 \mathrm{E}+00$ & $0.00 \mathrm{E}+00$ & $2.64 \mathrm{E}+00$ \\
\hline $7-21$ & 1De 3-3Po 5 & $5-1$ & 229.21 & $5.10 \mathrm{E}+00$ & $9.08 \mathrm{E}+00$ & $0.00 \mathrm{E}+00$ & $9.53 \mathrm{E}-01$ \\
\hline $9-21$ & 3Рe 3-3Рo 5 & $5-1$ & 241.50 & $5.30 \mathrm{E}+00$ & $9.08 \mathrm{E}+00$ & $0.00 \mathrm{E}+00$ & $6.45 \mathrm{E}+01$ \\
\hline $12-21$ & 3De 4-3Po 5 & $5-1$ & 316.36 & $6.19 E+00$ & $9.08 \mathrm{E}+00$ & $0.00 \mathrm{E}+00$ & $7.58 \mathrm{E}+00$ \\
\hline $13-21$ & 3De 4-3Po 5 & $7-1$ & 317.99 & $6.21 \mathrm{E}+00$ & $9.08 \mathrm{E}+00$ & $7.19 \mathrm{E}-02$ & $0.00 \mathrm{E}+00$ \\
\hline $14-21$ & 1De 4-3Po 5 & $5-1$ & 427.72 & $6.94 \mathrm{E}+00$ & $9.08 \mathrm{E}+00$ & $0.00 \mathrm{E}+00$ & $4.49 \mathrm{E}-01$ \\
\hline $13-22$ & 3De 4-3Po 5 & $7-3$ & 317.64 & $6.21 \mathrm{E}+00$ & $9.08 \mathrm{E}+00$ & $1.26 \mathrm{E}-02$ & $4.53 \mathrm{E}+00$ \\
\hline
\end{tabular}


Table 4. (continued)

\begin{tabular}{|c|c|c|c|c|c|c|c|}
\hline$i-j$ & $T_{i} C_{i}-T_{j} C_{j}$ & $g_{i}-g_{j}$ & $\lambda(\AA)$ & $E_{i}(\mathrm{Ry})$ & $E_{j}(\mathrm{Ry})$ & AE3 & AM2 \\
\hline $1-23$ & 1Se 1-3Do 5 & $1-7$ & 100.52 & $0.00 \mathrm{E}+00$ & $9.07 \mathrm{E}+00$ & $4.56 \mathrm{E}-02$ & $0.00 \mathrm{E}+00$ \\
\hline $6-23$ & 3Pe 3-3Do 5 & $1-7$ & 227.10 & $5.05 E+00$ & $9.07 \mathrm{E}+00$ & $1.92 \mathrm{E}-01$ & $0.00 \mathrm{E}+00$ \\
\hline $8-23$ & 3Pe 3-3Do 5 & $3-7$ & 232.42 & $5.14 \mathrm{E}+00$ & $9.07 \mathrm{E}+00$ & $3.71 \mathrm{E}-01$ & $1.85 \mathrm{E}+01$ \\
\hline $10-23$ & 1Se 3-3Do 5 & $1-7$ & 298.31 & $6.01 \mathrm{E}+00$ & $9.07 \mathrm{E}+00$ & $3.24 \mathrm{E}-04$ & $0.00 \mathrm{E}+00$ \\
\hline $11-23$ & 3De 4-3Do 5 & $3-7$ & 316.37 & $6.19 \mathrm{E}+00$ & $9.07 \mathrm{E}+00$ & $1.22 \mathrm{E}-02$ & $3.67 \mathrm{E}-01$ \\
\hline $1-24$ & 1Se 1-3Do 5 & $1-5$ & 100.34 & $0.00 \mathrm{E}+00$ & $9.08 \mathrm{E}+00$ & $0.00 \mathrm{E}+00$ & $1.43 \mathrm{E}+00$ \\
\hline $6-24$ & 3Pe 3-3Do 5 & $1-5$ & 226.19 & $5.05 \mathrm{E}+00$ & $9.08 \mathrm{E}+00$ & $0.00 \mathrm{E}+00$ & $4.17 \mathrm{E}+01$ \\
\hline $10-24$ & 1Se 3-3Do 5 & $1-5$ & 296.74 & $6.01 \mathrm{E}+00$ & $9.08 \mathrm{E}+00$ & $0.00 \mathrm{E}+00$ & $1.41 \mathrm{E}+00$ \\
\hline $1-25$ & 1Se $1-1$ Fo 5 & $1-7$ & 94.12 & $0.00 \mathrm{E}+00$ & $9.68 \mathrm{E}+00$ & $1.03 \mathrm{E}+01$ & $0.00 \mathrm{E}+00$ \\
\hline $6-25$ & 3Pe 3-1Fo 5 & $1-7$ & 196.85 & $5.05 \mathrm{E}+00$ & $9.68 \mathrm{E}+00$ & $4.02 \mathrm{E}-04$ & $0.00 \mathrm{E}+00$ \\
\hline $8-25$ & 3Pe 3-1Fo 5 & $3-7$ & 200.83 & $5.14 \mathrm{E}+00$ & $9.68 \mathrm{E}+00$ & $7.15 \mathrm{E}-03$ & $3.94 \mathrm{E}-01$ \\
\hline $10-25$ & 1Se $3-1$ Fo 5 & $1-7$ & 248.21 & $6.01 \mathrm{E}+00$ & $9.68 \mathrm{E}+00$ & $7.98 \mathrm{E}-02$ & $0.00 \mathrm{E}+00$ \\
\hline $11-25$ & 3De 4-1Fo 5 & $3-7$ & 260.59 & $6.19 E+00$ & $9.68 \mathrm{E}+00$ & $1.05 \mathrm{E}-05$ & $6.81 \mathrm{E}+00$ \\
\hline $13-26$ & 3De 4-1Po 5 & $7-3$ & 254.15 & $6.21 \mathrm{E}+00$ & $9.80 \mathrm{E}+00$ & $3.22 \mathrm{E}-03$ & $1.07 \mathrm{E}+01$ \\
\hline $2-27$ & 3Po 2-3Fe 19 & $1-5$ & 87.99 & $2.13 \mathrm{E}+00$ & $1.25 \mathrm{E}+01$ & $0.00 \mathrm{E}+00$ & $2.52 \mathrm{E}+00$ \\
\hline $17-27$ & 3Fo 5-3Fe 19 & $9-5$ & 237.74 & $8.65 E+00$ & $1.25 \mathrm{E}+01$ & $2.67 \mathrm{E}-02$ & $2.35 \mathrm{E}+00$ \\
\hline $21-27$ & 3Рo 5-3Fe 19 & $1-5$ & 267.09 & $9.08 \mathrm{E}+00$ & $1.25 \mathrm{E}+01$ & $0.00 \mathrm{E}+00$ & $3.12 \mathrm{E}+00$ \\
\hline $2-28$ & 3Po 2-3Fe 19 & $1-7$ & 87.86 & $2.13 \mathrm{E}+00$ & $1.25 \mathrm{E}+01$ & $4.45 \mathrm{E}-01$ & $0.00 \mathrm{E}+00$ \\
\hline $3-28$ & 3Рo 2-3Fe 19 & $3-7$ & 88.31 & $2.18 \mathrm{E}+00$ & $1.25 \mathrm{E}+01$ & $5.65 \mathrm{E}-02$ & $3.39 E+00$ \\
\hline $5-28$ & 1Po 2-3Fe 19 & $3-7$ & 98.03 & $3.21 \mathrm{E}+00$ & $1.25 \mathrm{E}+01$ & $2.12 \mathrm{E}-04$ & $3.24 \mathrm{E}-02$ \\
\hline $19-28$ & 3Do 5-3Fe 19 & $3-7$ & 256.96 & $8.96 \mathrm{E}+00$ & $1.25 \mathrm{E}+01$ & $1.21 \mathrm{E}-01$ & $3.70 \mathrm{E}+00$ \\
\hline $21-28$ & 3Po 5-3Fe 19 & $1-7$ & 265.85 & $9.08 \mathrm{E}+00$ & $1.25 \mathrm{E}+01$ & $1.36 \mathrm{E}-02$ & $0.00 \mathrm{E}+00$ \\
\hline $22-28$ & 3Po 5-3Fe 19 & $3-7$ & 266.09 & $9.08 \mathrm{E}+00$ & $1.25 \mathrm{E}+01$ & $1.94 \mathrm{E}-02$ & $1.16 \mathrm{E}+01$ \\
\hline $26-28$ & 1Po 5-3Fe 19 & $3-7$ & 336.52 & $9.80 \mathrm{E}+00$ & $1.25 \mathrm{E}+01$ & $1.77 \mathrm{E}-04$ & $6.96 \mathrm{E}-03$ \\
\hline $3-29$ & 3Po 2-3Fe 19 & $3-9$ & 88.16 & $2.18 \mathrm{E}+00$ & $1.25 \mathrm{E}+01$ & $5.16 \mathrm{E}-01$ & $0.00 \mathrm{E}+00$ \\
\hline $4-29$ & 3Po 2-3Fe 19 & $5-9$ & 89.27 & $2.31 \mathrm{E}+00$ & $1.25 E+01$ & $7.75 \mathrm{E}-01$ & $5.23 E+00$ \\
\hline $5-29$ & 1Po 2-3Fe 19 & $3-9$ & 97.84 & $3.21 \mathrm{E}+00$ & $1.25 \mathrm{E}+01$ & $1.17 \mathrm{E}-02$ & $0.00 \mathrm{E}+00$ \\
\hline $15-29$ & 3Fo 5-3Fe 19 & $5-9$ & 224.33 & $8.46 \mathrm{E}+00$ & $1.25 \mathrm{E}+01$ & $1.12 \mathrm{E}-02$ & $1.56 \mathrm{E}+01$ \\
\hline $18-29$ & 1Do 5-3Fe 19 & $5-9$ & 235.01 & $8.64 \mathrm{E}+00$ & $1.25 \mathrm{E}+01$ & $8.12 \mathrm{E}-05$ & $2.29 \mathrm{E}+01$ \\
\hline $19-29$ & 3Do 5-3Fe 19 & $3-9$ & 255.66 & $8.96 \mathrm{E}+00$ & $1.25 \mathrm{E}+01$ & $3.40 \mathrm{E}-02$ & $0.00 \mathrm{E}+00$ \\
\hline $20-29$ & 3Po 5-3Fe 19 & $5-9$ & 256.07 & $8.96 \mathrm{E}+00$ & $1.25 \mathrm{E}+01$ & $1.49 \mathrm{E}-01$ & $5.95 \mathrm{E}-01$ \\
\hline $22-29$ & 3Рo 5-3Fe 19 & $3-9$ & 264.70 & $9.08 \mathrm{E}+00$ & $1.25 \mathrm{E}+01$ & $2.33 \mathrm{E}-03$ & $0.00 \mathrm{E}+00$ \\
\hline $24-29$ & 3Do 5-3Fe 19 & $5-9$ & 264.97 & $9.08 \mathrm{E}+00$ & $1.25 \mathrm{E}+01$ & $1.98 \mathrm{E}-02$ & $1.27 \mathrm{E}+01$ \\
\hline $26-29$ & 1Po 5-3Fe 19 & $3-9$ & 334.30 & $9.80 \mathrm{E}+00$ & $1.25 \mathrm{E}+01$ & $2.47 \mathrm{E}-07$ & $0.00 \mathrm{E}+00$ \\
\hline $2-30$ & 3Рo 2-3Ре 19 & $1-5$ & 85.18 & $2.13 E+00$ & $1.28 \mathrm{E}+01$ & $0.00 \mathrm{E}+00$ & $1.52 \mathrm{E}-02$ \\
\hline $17-30$ & 3Fo 5-3Pe 19 & $9-5$ & 218.26 & $8.65 E+00$ & $1.28 \mathrm{E}+01$ & $1.13 \mathrm{E}-01$ & $7.45 \mathrm{E}+00$ \\
\hline $21-30$ & 3Рo 5-3Рe 19 & $1-5$ & 242.76 & $9.08 \mathrm{E}+00$ & $1.28 \mathrm{E}+01$ & $0.00 \mathrm{E}+00$ & $6.15 E+00$ \\
\hline $4-31$ & 3Рo 2-3Pe 19 & $5-1$ & 85.47 & $2.31 \mathrm{E}+00$ & $1.30 \mathrm{E}+01$ & $0.00 \mathrm{E}+00$ & $4.50 \mathrm{E}+00$ \\
\hline $15-31$ & 3Fo 5-3Pe 19 & $5-1$ & 201.80 & $8.46 \mathrm{E}+00$ & $1.30 \mathrm{E}+01$ & $0.00 \mathrm{E}+00$ & $8.60 \mathrm{E}+00$ \\
\hline $16-31$ & 3Fo 5-3Pe 19 & $7-1$ & 205.90 & $8.55 \mathrm{E}+00$ & $1.30 \mathrm{E}+01$ & $1.18 \mathrm{E}+00$ & $0.00 \mathrm{E}+00$ \\
\hline $18-31$ & 1Do 5-3Pe 19 & $5-1$ & 210.40 & $8.64 \mathrm{E}+00$ & $1.30 \mathrm{E}+01$ & $0.00 \mathrm{E}+00$ & $8.71 \mathrm{E}+00$ \\
\hline $20-31$ & 3Рo 5-3Pe 19 & $5-1$ & 227.13 & $8.96 \mathrm{E}+00$ & $1.30 \mathrm{E}+01$ & $0.00 \mathrm{E}+00$ & $1.16 \mathrm{E}+01$ \\
\hline $23-31$ & 3Do 5-3Pe 19 & $7-1$ & 233.13 & $9.07 \mathrm{E}+00$ & $1.30 \mathrm{E}+01$ & $4.99 \mathrm{E}-01$ & $0.00 \mathrm{E}+00$ \\
\hline $24-31$ & 3Do 5-3Pe 19 & $5-1$ & 234.10 & $9.08 \mathrm{E}+00$ & $1.30 \mathrm{E}+01$ & $0.00 \mathrm{E}+00$ & $8.27 E+01$ \\
\hline $25-31$ & 1Fo 5-3Pe 19 & $7-1$ & 276.80 & $9.68 \mathrm{E}+00$ & $1.30 \mathrm{E}+01$ & $2.04 \mathrm{E}-04$ & $0.00 \mathrm{E}+00$ \\
\hline $16-32$ & 3Fo 5-3Pe 19 & $7-3$ & 205.48 & $8.55 \mathrm{E}+00$ & $1.30 \mathrm{E}+01$ & $2.89 \mathrm{E}-02$ & $1.51 \mathrm{E}+00$ \\
\hline $17-32$ & 3Fo 5-3Pe 19 & $9-3$ & 210.47 & $8.65 \mathrm{E}+00$ & $1.30 \mathrm{E}+01$ & $5.78 \mathrm{E}-01$ & $0.00 \mathrm{E}+00$ \\
\hline $23-32$ & 3Do 5-3Pe 19 & $7-3$ & 232.59 & $9.07 \mathrm{E}+00$ & $1.30 \mathrm{E}+01$ & $2.89 \mathrm{E}-01$ & $3.96 \mathrm{E}+01$ \\
\hline $25-32$ & 1Fo 5-3Pe 19 & $7-3$ & 276.04 & $9.68 \mathrm{E}+00$ & $1.30 \mathrm{E}+01$ & $3.39 \mathrm{E}-04$ & $7.22 \mathrm{E}-02$ \\
\hline $2-33$ & 3Po 2-1De 19 & $1-5$ & 85.56 & $2.13 \mathrm{E}+00$ & $1.28 \mathrm{E}+01$ & $0.00 \mathrm{E}+00$ & $1.05 \mathrm{E}+00$ \\
\hline $17-33$ & 3Fo 5-1De 19 & $9-5$ & 220.80 & $8.65 E+00$ & $1.28 \mathrm{E}+01$ & $3.06 \mathrm{E}-01$ & $2.17 \mathrm{E}-01$ \\
\hline $21-33$ & 3Po 5-1De 19 & $1-5$ & 245.90 & $9.08 \mathrm{E}+00$ & $1.28 \mathrm{E}+01$ & $0.00 \mathrm{E}+00$ & $3.30 \mathrm{E}-02$ \\
\hline $3-34$ & 3Po 2-1Ge 19 & $3-9$ & 85.66 & $2.18 \mathrm{E}+00$ & $1.28 \mathrm{E}+01$ & $9.76 \mathrm{E}-02$ & $0.00 \mathrm{E}+00$ \\
\hline $4-34$ & 3Рo 2-1Ge 19 & $5-9$ & 86.71 & $2.31 \mathrm{E}+00$ & $1.28 \mathrm{E}+01$ & $3.14 \mathrm{E}-03$ & $7.28 \mathrm{E}-03$ \\
\hline $5-34$ & 1Po $2-1 \mathrm{Ge} 19$ & $3-9$ & 94.77 & $3.21 \mathrm{E}+00$ & $1.28 \mathrm{E}+01$ & $1.07 \mathrm{E}+01$ & $0.00 \mathrm{E}+00$ \\
\hline $15-34$ & 3Fo 5-1Ge 19 & $5-9$ & 208.85 & $8.46 \mathrm{E}+00$ & $1.28 \mathrm{E}+01$ & $8.30 \mathrm{E}-02$ & $7.09 \mathrm{E}+01$ \\
\hline $18-34$ & 1Do 5-1Ge 19 & $5-9$ & 218.08 & $8.64 \mathrm{E}+00$ & $1.28 \mathrm{E}+01$ & $4.75 \mathrm{E}-01$ & $2.51 \mathrm{E}+00$ \\
\hline $19-34$ & 3Do 5-1Ge 19 & $3-9$ & 235.75 & $8.96 \mathrm{E}+00$ & $1.28 \mathrm{E}+01$ & $1.80 \mathrm{E}-03$ & $0.00 \mathrm{E}+00$ \\
\hline $20-34$ & 3Рo 5-1Ge 19 & $5-9$ & 236.10 & $8.96 \mathrm{E}+00$ & $1.28 \mathrm{E}+01$ & $3.64 \mathrm{E}-03$ & $1.33 \mathrm{E}-02$ \\
\hline $22-34$ & 3Po 5-1Ge 19 & $3-9$ & 243.42 & $9.08 \mathrm{E}+00$ & $1.28 \mathrm{E}+01$ & $1.81 \mathrm{E}-06$ & $0.00 \mathrm{E}+00$ \\
\hline $24-34$ & 3Do 5-1Ge 19 & $5-9$ & 243.65 & $9.08 \mathrm{E}+00$ & $1.28 \mathrm{E}+01$ & $1.80 \mathrm{E}-03$ & $3.87 \mathrm{E}-01$ \\
\hline $26-34$ & 1Po 5-1Ge 19 & $3-9$ & 301.06 & $9.80 \mathrm{E}+00$ & $1.28 \mathrm{E}+01$ & $4.99 \mathrm{E}-02$ & $0.00 \mathrm{E}+00$ \\
\hline $4-35$ & 3Po 2-1Se 19 & $5-1$ & 81.09 & $2.31 \mathrm{E}+00$ & $1.36 \mathrm{E}+01$ & $0.00 \mathrm{E}+00$ & $6.26 \mathrm{E}-01$ \\
\hline $15-35$ & 3Fo 5-1Se 19 & $5-1$ & 178.95 & $8.46 \mathrm{E}+00$ & $1.36 \mathrm{E}+01$ & $0.00 \mathrm{E}+00$ & $8.14 \mathrm{E}+00$ \\
\hline $16-35$ & 3Fo 5-1Se 19 & $7-1$ & 182.17 & $8.55 \mathrm{E}+00$ & $1.36 \mathrm{E}+01$ & $3.10 \mathrm{E}-02$ & $0.00 \mathrm{E}+00$ \\
\hline $18-35$ & 1Do 5-1Se 19 & $5-1$ & 185.69 & $8.64 \mathrm{E}+00$ & $1.36 \mathrm{E}+01$ & $0.00 \mathrm{E}+00$ & $7.13 \mathrm{E}+01$ \\
\hline $20-35$ & 3Po 5-1Se 19 & $5-1$ & 198.59 & $8.96 \mathrm{E}+00$ & $1.36 \mathrm{E}+01$ & $0.00 \mathrm{E}+00$ & $3.64 \mathrm{E}+01$ \\
\hline $23-35$ & 3Do 5-1Se 19 & $7-1$ & 203.17 & $9.07 \mathrm{E}+00$ & $1.36 \mathrm{E}+01$ & $4.26 \mathrm{E}-03$ & $0.00 \mathrm{E}+00$ \\
\hline $24-35$ & 3Do 5-1Se 19 & $5-1$ & 203.90 & $9.08 \mathrm{E}+00$ & $1.36 \mathrm{E}+01$ & $0.00 \mathrm{E}+00$ & $3.93 \mathrm{E}+01$ \\
\hline $25-35$ & 1Fo 5-1Se 19 & $7-1$ & 235.55 & $9.68 \mathrm{E}+00$ & $1.36 \mathrm{E}+01$ & $1.02 \mathrm{E}+00$ & $0.00 \mathrm{E}+00$ \\
\hline $16-36$ & 3Fo 5-3Se 6 & $7-3$ & 121.13 & $8.55 \mathrm{E}+00$ & $1.61 \mathrm{E}+01$ & $8.69 \mathrm{E}-01$ & $1.25 \mathrm{E}-03$ \\
\hline $17-36$ & 3Fo 5-3Se 6 & $9-3$ & 122.84 & $8.65 \mathrm{E}+00$ & $1.61 \mathrm{E}+01$ & $1.03 \mathrm{E}+00$ & $0.00 \mathrm{E}+00$ \\
\hline $23-36$ & 3Do 5-3Se 6 & $7-3$ & 130.07 & $9.07 \mathrm{E}+00$ & $1.61 \mathrm{E}+01$ & $4.01 \mathrm{E}-03$ & $1.36 \mathrm{E}-01$ \\
\hline $25-36$ & 1Fo 5-3Se 6 & $7-3$ & 142.62 & $9.68 \mathrm{E}+00$ & $1.61 \mathrm{E}+01$ & $1.51 \mathrm{E}-04$ & $2.97 \mathrm{E}-04$ \\
\hline $4-37$ & 3Po 2-1Se 6 & $5-1$ & 65.23 & $2.31 \mathrm{E}+00$ & $1.63 E+01$ & $0.00 \mathrm{E}+00$ & $6.30 \mathrm{E}+03$ \\
\hline $15-37$ & 3Fo 5-1Se 6 & $5-1$ & 116.45 & $8.46 \mathrm{E}+00$ & $1.63 \mathrm{E}+01$ & $0.00 \mathrm{E}+00$ & $4.00 \mathrm{E}-02$ \\
\hline
\end{tabular}


Table 4. (continued)

\begin{tabular}{|c|c|c|c|c|c|c|c|}
\hline$i-j$ & $T_{i} C_{i}-T_{j} C_{j}$ & $g_{i}-g_{j}$ & $\lambda(\AA)$ & $E_{i}(\mathrm{Ry})$ & $E_{j}$ (Ry) & AE3 & AM2 \\
\hline $16-37$ & 3Fo 5-1Se 6 & $7-1$ & 117.81 & $8.55 \mathrm{E}+00$ & $1.63 \mathrm{E}+01$ & $5.12 \mathrm{E}-02$ & $0.00 \mathrm{E}+00$ \\
\hline $18-37$ & 1Do 5-1Se 6 & $5-1$ & 119.27 & $8.64 \mathrm{E}+00$ & $1.63 \mathrm{E}+01$ & $0.00 \mathrm{E}+00$ & $1.09 \mathrm{E}-01$ \\
\hline $20-37$ & 3Po 5-1Se 6 & $5-1$ & 124.46 & $8.96 \mathrm{E}+00$ & $1.63 \mathrm{E}+01$ & $0.00 \mathrm{E}+00$ & $9.00 \mathrm{E}-01$ \\
\hline $23-37$ & 3Do 5-1Se 6 & $7-1$ & 126.25 & $9.07 \mathrm{E}+00$ & $1.63 \mathrm{E}+01$ & $6.93 \mathrm{E}-02$ & $0.00 \mathrm{E}+00$ \\
\hline $24-37$ & 3Do 5-1Se 6 & $5-1$ & 126.53 & $9.08 \mathrm{E}+00$ & $1.63 \mathrm{E}+01$ & $0.00 \mathrm{E}+00$ & $5.51 \mathrm{E}-01$ \\
\hline $25-37$ & 1Fo 5-1Se 6 & $7-1$ & 138.04 & $9.68 \mathrm{E}+00$ & $1.63 \mathrm{E}+01$ & $6.39 \mathrm{E}+00$ & $0.00 \mathrm{E}+00$ \\
\hline $7-38$ & 1De 3-3Po 7 & $5-1$ & 75.30 & $5.10 \mathrm{E}+00$ & $1.72 \mathrm{E}+01$ & $0.00 \mathrm{E}+00$ & $2.32 \mathrm{E}+02$ \\
\hline $9-38$ & 3Рe 3-3Рo 7 & $5-1$ & 76.58 & $5.30 \mathrm{E}+00$ & $1.72 \mathrm{E}+01$ & $0.00 \mathrm{E}+00$ & $8.36 \mathrm{E}+01$ \\
\hline $12-38$ & 3De 4-3Po 7 & $5-1$ & 82.79 & $6.19 \mathrm{E}+00$ & $1.72 \mathrm{E}+01$ & $0.00 \mathrm{E}+00$ & $1.85 \mathrm{E}+03$ \\
\hline $13-38$ & 3De 4-3Po 7 & $7-1$ & 82.90 & $6.21 \mathrm{E}+00$ & $1.72 \mathrm{E}+01$ & $1.70 \mathrm{E}+03$ & $0.00 \mathrm{E}+00$ \\
\hline $14-38$ & 1De 4-3Po 7 & $5-1$ & 88.84 & $6.94 \mathrm{E}+00$ & $1.72 \mathrm{E}+01$ & $0.00 \mathrm{E}+00$ & $1.68 \mathrm{E}+02$ \\
\hline $27-38$ & 3Fe 19-3Ро 7 & $5-1$ & 193.29 & $1.25 \mathrm{E}+01$ & $1.72 \mathrm{E}+01$ & $0.00 \mathrm{E}+00$ & $1.70 \mathrm{E}-02$ \\
\hline $28-38$ & 3 Fe 19-3Po 7 & $7-1$ & 193.94 & $1.25 \mathrm{E}+01$ & $1.72 \mathrm{E}+01$ & $3.43 \mathrm{E}-04$ & $0.00 \mathrm{E}+00$ \\
\hline $30-38$ & 3Рe 19-3Ро 7 & $5-1$ & 208.40 & $1.28 \mathrm{E}+01$ & $1.72 \mathrm{E}+01$ & $0.00 \mathrm{E}+00$ & $2.02 \mathrm{E}-02$ \\
\hline $33-38$ & 1De 19-3Po 7 & $5-1$ & 206.14 & $1.28 \mathrm{E}+01$ & $1.72 \mathrm{E}+01$ & $0.00 \mathrm{E}+00$ & $1.23 \mathrm{E}-02$ \\
\hline $13-39$ & 3De 4-3Po 7 & $7-3$ & 82.86 & $6.21 \mathrm{E}+00$ & $1.72 \mathrm{E}+01$ & $9.02 \mathrm{E}+02$ & $2.15 \mathrm{E}+02$ \\
\hline $28-39$ & 3Fe 19-3Ро 7 & $7-3$ & 193.69 & $1.25 \mathrm{E}+01$ & $1.72 \mathrm{E}+01$ & $4.94 \mathrm{E}-06$ & $7.16 \mathrm{E}-03$ \\
\hline 29-39 & 3Fe 19-3Рo 7 & $9-3$ & 194.44 & $1.25 \mathrm{E}+01$ & $1.72 \mathrm{E}+01$ & $6.13 \mathrm{E}-05$ & $0.00 \mathrm{E}+00$ \\
\hline $34-39$ & 1Ge 19-3Рo 7 & $9-3$ & 207.78 & $1.28 \mathrm{E}+01$ & $1.72 \mathrm{E}+01$ & $1.38 \mathrm{E}-03$ & $0.00 \mathrm{E}+00$ \\
\hline $1-40$ & 1Se 1-3Po 7 & $1-5$ & 52.80 & $0.00 \mathrm{E}+00$ & $1.73 \mathrm{E}+01$ & $0.00 \mathrm{E}+00$ & $4.91 \mathrm{E}+03$ \\
\hline $6-40$ & 3Pe 3-3Po 7 & $1-5$ & 74.66 & $5.05 \mathrm{E}+00$ & $1.73 \mathrm{E}+01$ & $0.00 \mathrm{E}+00$ & $1.24 \mathrm{E}+00$ \\
\hline $10-40$ & 1Se 3-3Рo 7 & $1-5$ & 81.02 & $6.01 \mathrm{E}+00$ & $1.73 \mathrm{E}+01$ & $0.00 \mathrm{E}+00$ & $6.22 \mathrm{E}-01$ \\
\hline $29-40$ & 3 Fe $19-3$ Po 7 & $9-5$ & 192.34 & $1.25 \mathrm{E}+01$ & $1.73 \mathrm{E}+01$ & $1.28 \mathrm{E}-04$ & $1.09 \mathrm{E}-02$ \\
\hline $31-40$ & 3Рe 19-3Рo 7 & $1-5$ & 212.70 & $1.30 \mathrm{E}+01$ & $1.73 \mathrm{E}+01$ & $0.00 \mathrm{E}+00$ & $4.69 \mathrm{E}-04$ \\
\hline $34-40$ & 1Ge 19-3Рo 7 & $9-5$ & 205.38 & $1.28 \mathrm{E}+01$ & $1.73 \mathrm{E}+01$ & $7.56 \mathrm{E}-07$ & $9.70 \mathrm{E}-06$ \\
\hline $35-40$ & 1Se 19-3Po 7 & $1-5$ & 245.77 & $1.36 \mathrm{E}+01$ & $1.73 \mathrm{E}+01$ & $0.00 \mathrm{E}+00$ & $1.06 \mathrm{E}-01$ \\
\hline $37-40$ & 1Se 6-3Po 7 & $1-5$ & 934.76 & $1.63 \mathrm{E}+01$ & $1.73 \mathrm{E}+01$ & $0.00 \mathrm{E}+00$ & $8.74 \mathrm{E}-02$ \\
\hline $13-41$ & 3De 4-1Po 7 & $7-3$ & 82.74 & $6.21 \mathrm{E}+00$ & $1.72 \mathrm{E}+01$ & $2.37 \mathrm{E}+02$ & $1.21 \mathrm{E}+03$ \\
\hline $28-41$ & $3 \mathrm{Fe}$ 19-1Po 7 & $7-3$ & 193.07 & $1.25 \mathrm{E}+01$ & $1.72 \mathrm{E}+01$ & $6.21 \mathrm{E}-06$ & $1.89 \mathrm{E}-03$ \\
\hline $29-41$ & 3Fe 19-1Po 7 & $9-3$ & 193.80 & $1.25 \mathrm{E}+01$ & $1.72 \mathrm{E}+01$ & $1.56 \mathrm{E}-04$ & $0.00 \mathrm{E}+00$ \\
\hline $34-41$ & $1 \mathrm{Ge} 19-1 \mathrm{Po} 7$ & $9-3$ & 207.06 & $1.28 \mathrm{E}+01$ & $1.72 \mathrm{E}+01$ & $6.40 \mathrm{E}-03$ & $0.00 \mathrm{E}+00$ \\
\hline $16-42$ & 3Fo 5-3De 8 & $7-3$ & 91.47 & $8.55 E+00$ & $1.85 \mathrm{E}+01$ & $2.68 \mathrm{E}+01$ & $5.31 \mathrm{E}+01$ \\
\hline $17-42$ & 3Fo 5-3De 8 & $9-3$ & 92.45 & $8.65 E+00$ & $1.85 \mathrm{E}+01$ & $3.67 \mathrm{E}+00$ & $0.00 \mathrm{E}+00$ \\
\hline $23-42$ & 3Do 5-3De 8 & $7-3$ & 96.48 & $9.07 \mathrm{E}+00$ & $1.85 \mathrm{E}+01$ & $1.07 \mathrm{E}-01$ & $1.36 \mathrm{E}+00$ \\
\hline $25-42$ & 1Fo 5-3De 8 & $7-3$ & 103.22 & $9.68 \mathrm{E}+00$ & $1.85 \mathrm{E}+01$ & $9.30 \mathrm{E}-02$ & $3.70 \mathrm{E}+01$ \\
\hline $2-43$ & 3Po 2-3De 8 & $1-5$ & 55.61 & $2.13 E+00$ & $1.85 \mathrm{E}+01$ & $0.00 \mathrm{E}+00$ & $8.54 \mathrm{E}+03$ \\
\hline $17-43$ & 3Fo 5-3De 8 & $9-5$ & 92.39 & $8.65 E+00$ & $1.85 \mathrm{E}+01$ & $1.59 \mathrm{E}+01$ & $2.42 \mathrm{E}+01$ \\
\hline $21-43$ & 3Po 5-3De 8 & $1-5$ & 96.51 & $9.08 \mathrm{E}+00$ & $1.85 \mathrm{E}+01$ & $0.00 \mathrm{E}+00$ & $1.64 \mathrm{E}+00$ \\
\hline $38-43$ & 3Po 7-3De 8 & $1-5$ & 692.77 & $1.72 \mathrm{E}+01$ & $1.85 \mathrm{E}+01$ & $0.00 \mathrm{E}+00$ & $5.34 \mathrm{E}-01$ \\
\hline $2-44$ & 3Po 2-3De 8 & $1-7$ & 55.57 & $2.13 E+00$ & $1.85 \mathrm{E}+01$ & $6.24 \mathrm{E}+03$ & $0.00 \mathrm{E}+00$ \\
\hline $3-44$ & 3Po 2-3De 8 & $3-7$ & 55.76 & $2.18 \mathrm{E}+00$ & $1.85 \mathrm{E}+01$ & $1.23 \mathrm{E}+04$ & $6.68 \mathrm{E}+03$ \\
\hline $5-44$ & 1Po 2-3De 8 & $3-7$ & 59.48 & $3.21 \mathrm{E}+00$ & $1.85 \mathrm{E}+01$ & $5.96 \mathrm{E}+01$ & $3.73 E+03$ \\
\hline $19-44$ & 3Do 5-3De 8 & $3-7$ & 95.21 & $8.96 \mathrm{E}+00$ & $1.85 \mathrm{E}+01$ & $2.25 \mathrm{E}-01$ & $6.41 \mathrm{E}-01$ \\
\hline $21-44$ & 3Po 5-3De 8 & $1-7$ & 96.40 & $9.08 \mathrm{E}+00$ & $1.85 \mathrm{E}+01$ & $5.91 \mathrm{E}-02$ & $0.00 \mathrm{E}+00$ \\
\hline $22-44$ & 3Po 5-3De 8 & $3-7$ & 96.43 & $9.08 \mathrm{E}+00$ & $1.85 \mathrm{E}+01$ & $2.41 \mathrm{E}-02$ & $1.39 \mathrm{E}+00$ \\
\hline $26-44$ & 1Po 5-3De 8 & $3-7$ & 104.35 & $9.80 \mathrm{E}+00$ & $1.85 \mathrm{E}+01$ & $8.32 \mathrm{E}-03$ & $2.82 \mathrm{E}+00$ \\
\hline $38-44$ & 3Po 7-3De 8 & $1-7$ & 687.03 & $1.72 \mathrm{E}+01$ & $1.85 \mathrm{E}+01$ & $4.01 \mathrm{E}-03$ & $0.00 \mathrm{E}+00$ \\
\hline $39-44$ & 3Po 7-3De 8 & $3-7$ & 690.19 & $1.72 \mathrm{E}+01$ & $1.85 \mathrm{E}+01$ & $6.13 \mathrm{E}-03$ & $8.44 \mathrm{E}-02$ \\
\hline $41-44$ & 1Po 7-3De 8 & $3-7$ & 698.29 & $1.72 \mathrm{E}+01$ & $1.85 \mathrm{E}+01$ & $1.52 \mathrm{E}-03$ & $8.19 \mathrm{E}-01$ \\
\hline $2-45$ & 3Po 2-1De 8 & $1-5$ & 55.51 & $2.13 \mathrm{E}+00$ & $1.85 \mathrm{E}+01$ & $0.00 \mathrm{E}+00$ & $8.87 \mathrm{E}+02$ \\
\hline $17-45$ & 3Fo 5-1De 8 & $9-5$ & 92.11 & $8.65 E+00$ & $1.85 \mathrm{E}+01$ & $8.76 \mathrm{E}-01$ & $4.12 \mathrm{E}+01$ \\
\hline $21-45$ & 3Po 5-1De 8 & $1-5$ & 96.21 & $9.08 \mathrm{E}+00$ & $1.85 \mathrm{E}+01$ & $0.00 \mathrm{E}+00$ & $3.93 \mathrm{E}-03$ \\
\hline $38-45$ & 3Po 7-1De 8 & $1-5$ & 677.32 & $1.72 \mathrm{E}+01$ & $1.85 \mathrm{E}+01$ & $0.00 \mathrm{E}+00$ & $9.43 \mathrm{E}-02$ \\
\hline $7-46$ & 1De 3-3Po 13 & $5-1$ & 66.48 & $5.10 \mathrm{E}+00$ & $1.88 \mathrm{E}+01$ & $0.00 \mathrm{E}+00$ & $3.57 \mathrm{E}+03$ \\
\hline $9-46$ & 3Рe 3-3Рo 13 & $5-1$ & 67.48 & $5.30 \mathrm{E}+00$ & $1.88 \mathrm{E}+01$ & $0.00 \mathrm{E}+00$ & $3.97 \mathrm{E}+02$ \\
\hline $12-46$ & 3De 4-3Po 13 & $5-1$ & 72.25 & $6.19 \mathrm{E}+00$ & $1.88 \mathrm{E}+01$ & $0.00 \mathrm{E}+00$ & $3.77 \mathrm{E}+01$ \\
\hline $13-46$ & 3De 4-3Po 13 & $7-1$ & 72.34 & $6.21 \mathrm{E}+00$ & $1.88 \mathrm{E}+01$ & $4.63 \mathrm{E}+01$ & $0.00 \mathrm{E}+00$ \\
\hline $14-46$ & 1De 4-3Po 13 & $5-1$ & 76.82 & $6.94 \mathrm{E}+00$ & $1.88 \mathrm{E}+01$ & $0.00 \mathrm{E}+00$ & $4.13 \mathrm{E}+02$ \\
\hline $27-46$ & 3Fe 19-3Рo 13 & $5-1$ & 144.19 & $1.25 \mathrm{E}+01$ & $1.88 \mathrm{E}+01$ & $0.00 \mathrm{E}+00$ & $5.47 \mathrm{E}-03$ \\
\hline $28-46$ & 3Fe 19-3Po 13 & $7-1$ & 144.55 & $1.25 \mathrm{E}+01$ & $1.88 \mathrm{E}+01$ & $4.32 \mathrm{E}-01$ & $0.00 \mathrm{E}+00$ \\
\hline $30-46$ & 3Рe 19-3Рo 13 & $5-1$ & 152.44 & $1.28 \mathrm{E}+01$ & $1.88 \mathrm{E}+01$ & $0.00 \mathrm{E}+00$ & $1.79 \mathrm{E}+00$ \\
\hline $33-46$ & 1De 19-3Po 13 & $5-1$ & 151.22 & $1.28 \mathrm{E}+01$ & $1.88 \mathrm{E}+01$ & $0.00 \mathrm{E}+00$ & $5.41 \mathrm{E}-03$ \\
\hline $43-46$ & 3De 8-3Рo 13 & $5-1$ & 3141 & $1.85 \mathrm{E}+01$ & $1.88 \mathrm{E}+01$ & $0.00 \mathrm{E}+00$ & $1.25 \mathrm{E}-06$ \\
\hline $44-46$ & 3De 8-3Po 13 & $7-1$ & 3265 & $1.85 \mathrm{E}+01$ & $1.88 \mathrm{E}+01$ & $3.17 \mathrm{E}-09$ & $0.00 \mathrm{E}+00$ \\
\hline $45-46$ & 1De 8-3Po 13 & $5-1$ & 3504 & $1.85 \mathrm{E}+01$ & $1.88 \mathrm{E}+01$ & $0.00 \mathrm{E}+00$ & $8.61 \mathrm{E}-07$ \\
\hline $13-47$ & 3De 4-3Po 13 & $7-3$ & 72.15 & $6.21 \mathrm{E}+00$ & $1.88 \mathrm{E}+01$ & $2.86 \mathrm{E}+01$ & $4.37 \mathrm{E}+01$ \\
\hline $28-47$ & 3Fe 19-3Рo 13 & $7-3$ & 143.80 & $1.25 \mathrm{E}+01$ & $1.88 \mathrm{E}+01$ & $1.63 \mathrm{E}-02$ & $2.60 \mathrm{E}-04$ \\
\hline $29-47$ & $3 \mathrm{Fe}$ 19-3Po 13 & $9-3$ & 144.21 & $1.25 \mathrm{E}+01$ & $1.88 \mathrm{E}+01$ & $2.15 \mathrm{E}-01$ & $0.00 \mathrm{E}+00$ \\
\hline $34-47$ & 1Ge 19-3Рo 13 & $9-3$ & 151.42 & $1.28 \mathrm{E}+01$ & $1.88 \mathrm{E}+01$ & $1.93 \mathrm{E}-01$ & $0.00 \mathrm{E}+00$ \\
\hline $44-47$ & 3De 8-3Рo 13 & $7-3$ & 2920 & $1.85 \mathrm{E}+01$ & $1.88 \mathrm{E}+01$ & $4.12 \mathrm{E}-09$ & $5.30 \mathrm{E}-07$ \\
\hline $1-48$ & 1Se 1 -3Po 13 & $1-5$ & 48.01 & $0.00 \mathrm{E}+00$ & $1.90 \mathrm{E}+01$ & $0.00 \mathrm{E}+00$ & $6.60 \mathrm{E}-06$ \\
\hline $6-48$ & 3Рe 3-3Рo 13 & $1-5$ & 65.44 & $5.05 \mathrm{E}+00$ & $1.90 \mathrm{E}+01$ & $0.00 \mathrm{E}+00$ & $2.50 \mathrm{E}+02$ \\
\hline $10-48$ & 1Se 3-3Po 13 & $1-5$ & 70.27 & $6.01 \mathrm{E}+00$ & $1.90 \mathrm{E}+01$ & $0.00 \mathrm{E}+00$ & $5.31 \mathrm{E}+02$ \\
\hline $29-48$ & 3Fe 19-3Po 13 & $9-5$ & 141.10 & $1.25 \mathrm{E}+01$ & $1.90 \mathrm{E}+01$ & $2.44 \mathrm{E}-01$ & $1.59 \mathrm{E}-04$ \\
\hline $31-48$ & 3Pe 19-3Рo 13 & $1-5$ & 151.76 & $1.30 \mathrm{E}+01$ & $1.90 \mathrm{E}+01$ & $0.00 \mathrm{E}+00$ & $7.79 \mathrm{E}-02$ \\
\hline $34-48$ & $1 \mathrm{Ge} 19-3 \mathrm{Po} 13$ & $9-5$ & 148.00 & $1.28 \mathrm{E}+01$ & $1.90 \mathrm{E}+01$ & $3.18 \mathrm{E}-04$ & $1.12 \mathrm{E}-05$ \\
\hline
\end{tabular}


Table 4. (continued)

\begin{tabular}{|c|c|c|c|c|c|c|c|}
\hline$i-j$ & $T_{i} C_{i}-T_{j} C_{j}$ & $g_{i}-g_{j}$ & $\lambda(\AA)$ & $E_{i}(\mathrm{Ry})$ & $E_{j}(\mathrm{Ry})$ & AE3 & AM2 \\
\hline $35-48$ & 1Se 19-3Рo 13 & $1-5$ & 167.87 & $1.36 \mathrm{E}+01$ & $1.90 \mathrm{E}+01$ & $0.00 \mathrm{E}+00$ & $1.35 \mathrm{E}-01$ \\
\hline $37-48$ & 1Se 6-3Po 13 & $1-5$ & 338.09 & $1.63 E+01$ & $1.90 \mathrm{E}+01$ & $0.00 \mathrm{E}+00$ & $2.96 \mathrm{E}+00$ \\
\hline $13-49$ & 3De 4-1Po 13 & $7-3$ & 70.62 & $6.21 \mathrm{E}+00$ & $1.91 \mathrm{E}+01$ & $2.00 \mathrm{E}+00$ & $6.26 \mathrm{E}+01$ \\
\hline $28-49$ & 3Fe 19-1Po 13 & $7-3$ & 137.87 & $1.25 \mathrm{E}+01$ & $1.91 \mathrm{E}+01$ & $2.41 \mathrm{E}-03$ & $2.13 \mathrm{E}-06$ \\
\hline $29-49$ & 3Fe 19-1Po 13 & $9-3$ & 138.25 & $1.25 \mathrm{E}+01$ & $1.91 \mathrm{E}+01$ & $5.50 \mathrm{E}-03$ & $0.00 \mathrm{E}+00$ \\
\hline $34-49$ & 1Ge 19-1Po 13 & $9-3$ & 144.87 & $1.28 \mathrm{E}+01$ & $1.91 \mathrm{E}+01$ & $2.72 \mathrm{E}+00$ & $0.00 \mathrm{E}+00$ \\
\hline $44-49$ & 3De 8-1Po 13 & $7-3$ & 1559 & $1.85 \mathrm{E}+01$ & $1.91 \mathrm{E}+01$ & $6.64 \mathrm{E}-08$ & $2.61 \mathrm{E}-04$ \\
\hline $1-50$ & 1Se $1-3$ Fo 9 & $1-5$ & 47.43 & $0.00 \mathrm{E}+00$ & $1.92 \mathrm{E}+01$ & $0.00 \mathrm{E}+00$ & $8.96 \mathrm{E}-04$ \\
\hline $6-50$ & 3Pe 3-3Fo 9 & $1-5$ & 64.35 & $5.05 \mathrm{E}+00$ & $1.92 \mathrm{E}+01$ & $0.00 \mathrm{E}+00$ & $1.09 \mathrm{E}+00$ \\
\hline $10-50$ & 1Se 3-3Fo 9 & $1-5$ & 69.02 & $6.01 E+00$ & $1.92 \mathrm{E}+01$ & $0.00 \mathrm{E}+00$ & $7.30 \mathrm{E}-03$ \\
\hline $29-50$ & 3Fe 19-3Fo 9 & $9-5$ & 136.15 & $1.25 \mathrm{E}+01$ & $1.92 \mathrm{E}+01$ & $4.89 \mathrm{E}-04$ & $9.55 \mathrm{E}-03$ \\
\hline $31-50$ & 3Pe 19-3Fo 9 & $1-5$ & 146.05 & $1.30 \mathrm{E}+01$ & $1.92 \mathrm{E}+01$ & $0.00 \mathrm{E}+00$ & $6.08 \mathrm{E}-03$ \\
\hline $34-50$ & 1Ge $19-3$ Fo 9 & $9-5$ & 142.56 & $1.28 \mathrm{E}+01$ & $1.92 \mathrm{E}+01$ & $2.09 \mathrm{E}-05$ & $6.94 \mathrm{E}-01$ \\
\hline $35-50$ & 1Se 19-3Fo 9 & $1-5$ & 160.92 & $1.36 \mathrm{E}+01$ & $1.92 \mathrm{E}+01$ & $0.00 \mathrm{E}+00$ & $4.62 \mathrm{E}-06$ \\
\hline $37-50$ & 1Se 6-3Fo 9 & $1-5$ & 311.01 & $1.63 \mathrm{E}+01$ & $1.92 \mathrm{E}+01$ & $0.00 \mathrm{E}+00$ & $2.17 \mathrm{E}-06$ \\
\hline $1-51$ & 1Se $1-3$ Fo 9 & $1-7$ & 47.42 & $0.00 \mathrm{E}+00$ & $1.92 \mathrm{E}+01$ & $1.10 \mathrm{E}+01$ & $0.00 \mathrm{E}+00$ \\
\hline $6-51$ & 3Pe 3-3Fo 9 & $1-7$ & 64.35 & $5.05 \mathrm{E}+00$ & $1.92 \mathrm{E}+01$ & $7.90 \mathrm{E}+00$ & $0.00 \mathrm{E}+00$ \\
\hline $8-51$ & 3Pe 3-3Fo 9 & $3-7$ & 64.77 & $5.14 \mathrm{E}+00$ & $1.92 \mathrm{E}+01$ & $1.57 \mathrm{E}+00$ & $1.19 \mathrm{E}+00$ \\
\hline $10-51$ & 1Se 3-3Fo 9 & $1-7$ & 69.01 & $6.01 E+00$ & $1.92 \mathrm{E}+01$ & $3.20 \mathrm{E}-02$ & $0.00 \mathrm{E}+00$ \\
\hline $11-51$ & 3De 4-3Fo 9 & $3-7$ & 69.94 & $6.19 E+00$ & $1.92 \mathrm{E}+01$ & $2.36 \mathrm{E}+03$ & $1.76 \mathrm{E}+04$ \\
\hline $31-51$ & 3Pe 19-3Fo 9 & $1-7$ & 146.03 & $1.30 \mathrm{E}+01$ & $1.92 \mathrm{E}+01$ & $2.90 \mathrm{E}-03$ & $0.00 \mathrm{E}+00$ \\
\hline $32-51$ & 3Pe 19-3Fo 9 & $3-7$ & 146.24 & $1.30 \mathrm{E}+01$ & $1.92 \mathrm{E}+01$ & $4.92 \mathrm{E}-04$ & $8.84 \mathrm{E}-03$ \\
\hline $35-51$ & 1Se 19-3Fo 9 & $1-7$ & 160.89 & $1.36 \mathrm{E}+01$ & $1.92 \mathrm{E}+01$ & $6.12 \mathrm{E}-05$ & $0.00 \mathrm{E}+00$ \\
\hline $36-51$ & 3Se 6-3Fo 9 & $3-7$ & 289.94 & $1.61 \mathrm{E}+01$ & $1.92 \mathrm{E}+01$ & $1.60 \mathrm{E}+00$ & $6.35 \mathrm{E}-07$ \\
\hline $37-51$ & 1Se 6-3Fo 9 & $1-7$ & 310.91 & $1.63 \mathrm{E}+01$ & $1.92 \mathrm{E}+01$ & $1.53 \mathrm{E}-04$ & $0.00 \mathrm{E}+00$ \\
\hline $42-51$ & 3De 8-3Fo 9 & $3-7$ & 1294 & $1.85 \mathrm{E}+01$ & $1.92 \mathrm{E}+01$ & $5.20 \mathrm{E}-05$ & $2.48 \mathrm{E}-02$ \\
\hline $7-52$ & 1De 3-3Fo 9 & $5-9$ & 64.54 & $5.10 \mathrm{E}+00$ & $1.92 \mathrm{E}+01$ & $1.16 \mathrm{E}+00$ & $2.01 \mathrm{E}+03$ \\
\hline $8-52$ & 3Pe 3-3Fo 9 & $3-9$ & 64.75 & $5.14 \mathrm{E}+00$ & $1.92 \mathrm{E}+01$ & $6.72 \mathrm{E}+00$ & $0.00 \mathrm{E}+00$ \\
\hline $9-52$ & 3Pe 3-3Fo 9 & $5-9$ & 65.48 & $5.30 \mathrm{E}+00$ & $1.92 \mathrm{E}+01$ & $1.12 \mathrm{E}+01$ & $3.54 \mathrm{E}+02$ \\
\hline $11-52$ & 3De 4-3Fo 9 & $3-9$ & 69.92 & $6.19 \mathrm{E}+00$ & $1.92 \mathrm{E}+01$ & $2.63 \mathrm{E}+02$ & $0.00 \mathrm{E}+00$ \\
\hline $12-52$ & 3De 4-3Fo 9 & $5-9$ & 69.97 & $6.19 \mathrm{E}+00$ & $1.92 \mathrm{E}+01$ & $2.18 \mathrm{E}+03$ & $1.42 \mathrm{E}+04$ \\
\hline $14-52$ & 1De 4-3Fo 9 & $5-9$ & 74.25 & $6.94 \mathrm{E}+00$ & $1.92 \mathrm{E}+01$ & $7.33 \mathrm{E}-01$ & $4.97 \mathrm{E}+03$ \\
\hline $27-52$ & 3Fe 19-3Fo 9 & $5-9$ & 135.38 & $1.25 \mathrm{E}+01$ & $1.92 \mathrm{E}+01$ & $2.46 \mathrm{E}-04$ & $1.14 \mathrm{E}-02$ \\
\hline $30-52$ & 3Рe 19-3Fo 9 & $5-9$ & 142.63 & $1.28 \mathrm{E}+01$ & $1.92 \mathrm{E}+01$ & $1.67 \mathrm{E}-03$ & $1.85 \mathrm{E}+00$ \\
\hline $32-52$ & 3Pe 19-3Fo 9 & $3-9$ & 146.17 & $1.30 \mathrm{E}+01$ & $1.92 \mathrm{E}+01$ & $2.90 \mathrm{E}-03$ & $0.00 \mathrm{E}+00$ \\
\hline $33-52$ & 1De 19-3Fo 9 & $5-9$ & 141.57 & $1.28 \mathrm{E}+01$ & $1.92 \mathrm{E}+01$ & $4.83 \mathrm{E}-03$ & $9.80 \mathrm{E}-01$ \\
\hline $36-52$ & 3 Se $6-3$ Fo 9 & $3-9$ & 289.66 & $1.61 \mathrm{E}+01$ & $1.92 \mathrm{E}+01$ & $1.61 \mathrm{E}+00$ & $0.00 \mathrm{E}+00$ \\
\hline $42-52$ & 3De 8-3Fo 9 & $3-9$ & 1288 & $1.85 \mathrm{E}+01$ & $1.92 \mathrm{E}+01$ & $5.96 \mathrm{E}-06$ & $0.00 \mathrm{E}+00$ \\
\hline $43-52$ & 3De 8-3Fo 9 & $5-9$ & 1299 & $1.85 \mathrm{E}+01$ & $1.92 \mathrm{E}+01$ & $4.56 \mathrm{E}-05$ & $1.46 \mathrm{E}-02$ \\
\hline $45-52$ & 1De 8-3Fo 9 & $5-9$ & 1358 & $1.85 \mathrm{E}+01$ & $1.92 \mathrm{E}+01$ & $9.24 \mathrm{E}-07$ & $1.54 \mathrm{E}-02$ \\
\hline $1-53$ & 1Se 1-1Fo 9 & $1-7$ & 47.10 & $0.00 \mathrm{E}+00$ & $1.93 \mathrm{E}+01$ & $7.04 \mathrm{E}+04$ & $0.00 \mathrm{E}+00$ \\
\hline $6-53$ & 3Pe 3-1Fo 9 & $1-7$ & 63.75 & $5.05 \mathrm{E}+00$ & $1.93 \mathrm{E}+01$ & $6.60 \mathrm{E}+00$ & $0.00 \mathrm{E}+00$ \\
\hline $8-53$ & 3Pe 3-1Fo 9 & $3-7$ & 64.16 & $5.14 \mathrm{E}+00$ & $1.93 E+01$ & $1.41 \mathrm{E}-01$ & $8.59 \mathrm{E}-02$ \\
\hline $10-53$ & 1Se 3-1Fo 9 & $1-7$ & 68.33 & $6.01 \mathrm{E}+00$ & $1.93 \mathrm{E}+01$ & $1.20 \mathrm{E}+02$ & $0.00 \mathrm{E}+00$ \\
\hline $11-53$ & 3De 4-1Fo 9 & $3-7$ & 69.23 & $6.19 \mathrm{E}+00$ & $1.93 \mathrm{E}+01$ & $3.92 \mathrm{E}-01$ & $4.19 E+03$ \\
\hline $31-53$ & 3Pe 19-1Fo 9 & $1-7$ & 142.98 & $1.30 \mathrm{E}+01$ & $1.93 \mathrm{E}+01$ & $1.30 \mathrm{E}-03$ & $0.00 \mathrm{E}+00$ \\
\hline $32-53$ & 3Pe 19-1Fo 9 & $3-7$ & 143.18 & $1.30 \mathrm{E}+01$ & $1.93 \mathrm{E}+01$ & $1.36 \mathrm{E}-05$ & $8.48 \mathrm{E}-05$ \\
\hline $35-53$ & 1Se 19-1Fo 9 & $1-7$ & 157.20 & $1.36 \mathrm{E}+01$ & $1.93 \mathrm{E}+01$ & $5.19 \mathrm{E}-01$ & $0.00 \mathrm{E}+00$ \\
\hline $36-53$ & 3Se 6-1Fo 9 & $3-7$ & 278.16 & $1.61 \mathrm{E}+01$ & $1.93 \mathrm{E}+01$ & $3.83 \mathrm{E}-04$ & $2.17 \mathrm{E}-06$ \\
\hline $37-53$ & 1Se 6-1Fo 9 & $1-7$ & 297.41 & $1.63 \mathrm{E}+01$ & $1.93 \mathrm{E}+01$ & $1.43 \mathrm{E}+00$ & $0.00 \mathrm{E}+00$ \\
\hline $42-53$ & 3De 8-1Fo 9 & $3-7$ & 1088 & $1.85 \mathrm{E}+01$ & $1.93 E+01$ & $2.74 \mathrm{E}-08$ & $2.00 \mathrm{E}-02$ \\
\hline $16-54$ & 3Fo 5-1Pe 14 & $7-3$ & 81.57 & $8.55 \mathrm{E}+00$ & $1.97 \mathrm{E}+01$ & $1.79 \mathrm{E}+02$ & $1.66 \mathrm{E}+02$ \\
\hline $17-54$ & 3Fo 5-1Pe 14 & $9-3$ & 82.35 & $8.65 E+00$ & $1.97 \mathrm{E}+01$ & $7.42 \mathrm{E}+01$ & $0.00 \mathrm{E}+00$ \\
\hline $23-54$ & 3Do 5-1Pe 14 & $7-3$ & 85.53 & $9.07 \mathrm{E}+00$ & $1.97 \mathrm{E}+01$ & $1.38 \mathrm{E}+00$ & $5.45 \mathrm{E}+02$ \\
\hline $25-54$ & 1Fo 5-1Pe 14 & $7-3$ & 90.78 & $9.68 \mathrm{E}+00$ & $1.97 \mathrm{E}+01$ & $2.31 \mathrm{E}+02$ & $2.06 \mathrm{E}+02$ \\
\hline $51-54$ & 3Fo 9-1Pe 14 & $7-3$ & 1804 & $1.92 \mathrm{E}+01$ & $1.97 \mathrm{E}+01$ & $4.30 \mathrm{E}-08$ & $6.80 \mathrm{E}-05$ \\
\hline $52-54$ & 3Fo 9-1Pe 14 & $9-3$ & 1815 & $1.92 \mathrm{E}+01$ & $1.97 \mathrm{E}+01$ & $1.92 \mathrm{E}-08$ & $0.00 \mathrm{E}+00$ \\
\hline $53-54$ & 1Fo 9-1Pe 14 & $7-3$ & 2449 & $1.93 \mathrm{E}+01$ & $1.97 \mathrm{E}+01$ & $9.81 \mathrm{E}-09$ & $5.92 \mathrm{E}-07$ \\
\hline $16-55$ & 3Fo 5-3De 14 & $7-3$ & 80.71 & $8.55 E+00$ & $1.98 \mathrm{E}+01$ & $9.65 \mathrm{E}+02$ & $6.41 \mathrm{E}+02$ \\
\hline $17-55$ & 3 Fo 5-3De 14 & $9-3$ & 81.47 & $8.65 \mathrm{E}+00$ & $1.98 \mathrm{E}+01$ & $1.03 \mathrm{E}+02$ & $0.00 \mathrm{E}+00$ \\
\hline $23-55$ & 3Do 5-3De 14 & $7-3$ & 84.58 & $9.07 \mathrm{E}+00$ & $1.98 \mathrm{E}+01$ & $1.94 \mathrm{E}+02$ & $1.03 \mathrm{E}+02$ \\
\hline $25-55$ & 1Fo 5-3De 14 & $7-3$ & 89.72 & $9.68 \mathrm{E}+00$ & $1.98 \mathrm{E}+01$ & $7.11 \mathrm{E}+01$ & $5.09 \mathrm{E}+01$ \\
\hline $51-55$ & 3Fo 9-3De 14 & $7-3$ & 1458 & $1.92 \mathrm{E}+01$ & $1.98 \mathrm{E}+01$ & $2.59 \mathrm{E}-07$ & $1.33 \mathrm{E}-04$ \\
\hline $52-55$ & 3Fo 9-3De 14 & $9-3$ & 1465 & $1.92 \mathrm{E}+01$ & $1.98 \mathrm{E}+01$ & $8.11 \mathrm{E}-09$ & $0.00 \mathrm{E}+00$ \\
\hline $53-55$ & 1Fo 9-3De 14 & $7-3$ & 1853 & $1.93 \mathrm{E}+01$ & $1.98 \mathrm{E}+01$ & $3.66 \mathrm{E}-08$ & $4.72 \mathrm{E}-05$ \\
\hline $2-56$ & 3Po 2-3De 14 & $1-5$ & 51.39 & $2.13 E+00$ & $1.99 \mathrm{E}+01$ & $0.00 \mathrm{E}+00$ & $1.57 \mathrm{E}+02$ \\
\hline $17-56$ & 3 Fo 5-3De 14 & $9-5$ & 81.29 & $8.65 \mathrm{E}+00$ & $1.99 \mathrm{E}+01$ & $8.00 \mathrm{E}+02$ & $7.15 E+02$ \\
\hline $21-56$ & 3Po 5-3De 14 & $1-5$ & 84.46 & $9.08 \mathrm{E}+00$ & $1.99 \mathrm{E}+01$ & $0.00 \mathrm{E}+00$ & $5.31 \mathrm{E}+00$ \\
\hline $38-56$ & 3Po 7-3De 14 & $1-5$ & 342.28 & $1.72 \mathrm{E}+01$ & $1.99 \mathrm{E}+01$ & $0.00 \mathrm{E}+00$ & $3.48 \mathrm{E}+00$ \\
\hline $46-56$ & 3Po 13-3De 14 & $1-5$ & 862.21 & $1.88 \mathrm{E}+01$ & $1.99 \mathrm{E}+01$ & $0.00 \mathrm{E}+00$ & $3.10 \mathrm{E}-02$ \\
\hline $52-56$ & 3Fo 9-3De 14 & $9-5$ & 1410 & $1.92 \mathrm{E}+01$ & $1.99 \mathrm{E}+01$ & $2.35 \mathrm{E}-07$ & $3.07 \mathrm{E}-04$ \\
\hline $4-57$ & 3Рo 2-3Pe 14 & $5-1$ & 51.82 & $2.31 \mathrm{E}+00$ & $1.99 \mathrm{E}+01$ & $0.00 \mathrm{E}+00$ & $9.97 \mathrm{E}+02$ \\
\hline $15-57$ & 3Fo 5-3Pe 14 & $5-1$ & 79.66 & $8.46 \mathrm{E}+00$ & $1.99 \mathrm{E}+01$ & $0.00 \mathrm{E}+00$ & $4.79 \mathrm{E}+02$ \\
\hline
\end{tabular}

